\title{
Guidelines for $\beta$-Lactam Synthesis: Glycal Protecting Groups Dictate Stereoelectronics and [2+2] Cycloaddition Kinetics
}

\author{
Anant S. Balijepalli ${ }^{1, \dagger}$ James H. McNeely ${ }^{2, \dagger}$, Aladin Hamoud ${ }^{2}$, and Mark W. Grinstaff ${ }^{1,2, *}$
}

1.) Department of Biomedical Engineering, Boston University, Boston, MA, 02215

2.) Department of Chemistry, Boston University, Boston, MA, 02215

\section{Table of Contents}

Supplementary Figures...................................................................................... S7

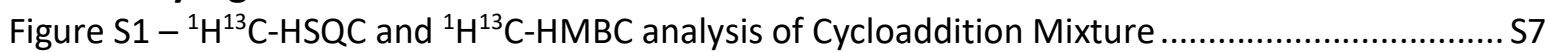

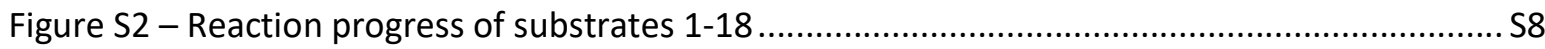

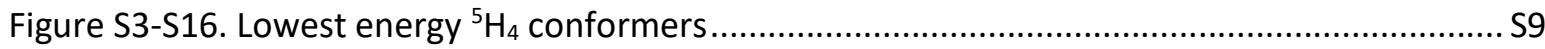

Figure S3. Lowest energy ${ }^{5} \mathrm{H}_{4}$ conformer [PBE0/DEF2-TZVP/SMD(ACN)] of 1 with ring atoms labeled with natural charges. HOMO isosurface shown on the right.......................................................... S9 Figure S4. Lowest energy ${ }^{5} \mathrm{H}_{4}$ conformer [PBE0/DEF2-TZVP/SMD(ACN)] of 2 with ring atoms labeled with natural charges. HOMO isosurface shown on the right ..................................................... S9 Figure S5. Lowest energy ${ }^{5} \mathrm{H}_{4}$ conformer [PBE0/DEF2-TZVP/SMD(ACN)] of 3 with ring atoms labeled with natural charges. HOMO isosurface shown on the right................................................... S10 Figure S6. Lowest energy ${ }^{5} \mathrm{H}_{4}$ conformer [PBE0/DEF2-TZVP/SMD(ACN)] of 4 with ring atoms labeled with natural charges. HOMO isosurface shown on the right...................................................... S10 Figure S7. Lowest energy ${ }^{5} \mathrm{H}_{4}$ conformer [PBE0/DEF2-TZVP/SMD(ACN)] of 5 with ring atoms labeled with natural charges. HOMO isosurface shown on the right.................................................... S11 Figure S8. Lowest energy $5 \mathrm{H} 4$ conformer [PBE0/DEF2-TZVP/SMD(ACN)] of 6 with ring atoms labeled with natural charges. HOMO-3 isosurface shown on the right.

Figure S9. Lowest energy ${ }^{5} \mathrm{H}_{4}$ conformer [PBE0/DEF2-TZVP/SMD(ACN)] of 7 with ring atoms labeled with natural charges. $\mathrm{HOMO}$ isosurface shown on the right. S12

Figure S10. Lowest energy ${ }^{5} \mathrm{H}_{4}$ conformer [PBE0/DEF2-TZVP/SMD(ACN)] of 9 with ring atoms labeled with natural charges. HOMO isosurface shown on the right.

Figure S11. Lowest energy ${ }^{5} \mathrm{H}_{4}$ conformer [PBEO/DEF2-TZVP/SMD(ACN)] of 10 with ring atoms labeled with natural charges. HOMO isosurface shown on the right.

Figure S12. Lowest energy ${ }^{5} \mathrm{H}_{4}$ conformer [PBE0/DEF2-TZVP/SMD(ACN)] of 11 with ring atoms labeled with natural charges. HOMO-2 isosurface shown on the right.

Figure S13. Lowest energy ${ }^{5} \mathrm{H}_{4}$ conformer [PBE0/DEF2-TZVP/SMD(ACN)] of 12 with ring atoms labeled with natural charges. HOMO isosurface shown on the right.

Figure S14. Lowest energy ${ }^{5} \mathrm{H}_{4}$ conformer [PBE0/DEF2-TZVP/SMD(ACN)] of 13 with ring atoms labeled with natural charges. HOMO isosurface shown on the right.

Figure S15. Lowest energy ${ }^{5} \mathrm{H}_{4}$ conformer [PBE0/DEF2-TZVP/SMD(ACN)] of 14 with ring atoms labeled with natural charges. HOMO isosurface shown on the right.

Figure S16. Lowest energy ${ }^{5} \mathrm{H}_{4}$ conformer [PBE0/DEF2-TZVP/SMD(ACN)] of 15 with ring atoms labeled with natural charges. HOMO isosurface shown on the right.

Figure S17. Comparison of correlation between ${ }^{5} \mathrm{H}_{4}$ and ${ }^{4} \mathrm{H}_{5}$ conformers 
Figure S17 - Comparison of correlation for pseudo-axially-oriented groups versus pseudoequatorially-oriented groups.

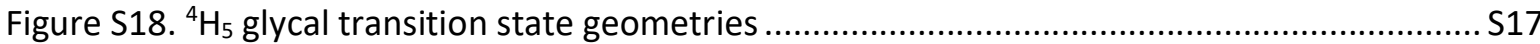

Figure S18 - Displaced geometries, scaled by 0.5 , along competing normal modes for the cycoaddition of ${ }^{4} \mathrm{H}_{5}$ with TCAI.

Figure S19. Comparison of ${ }^{5} \mathrm{H}_{4}$ IRC between gas phase and implicit solvation model ................... S18

Figure S19 - Portion of the IRC for 3 calculated at the PBE0/DEF2-SVP/D3BJ/RIJCOSX, PBE0/DEF2SVP/D3BJ/SMD(CH $\left.{ }_{3} \mathrm{CN}\right) / \mathrm{RIJCOSX}$, and PBE0/DEF2-SVP/D3BJ/SMD $\left(\mathrm{CH}_{3} \mathrm{CN}\right) / \mathrm{RIJCOSX//PBEO/DEF2-}$ SVP/D3BJ/RIJCOSX levels.

Figure S20. Comparison of IRC for selected glycal ${ }^{5} \mathrm{H}_{4}$ conformers.

Figure $\mathrm{S} 20$ - IRC of the ${ }^{5} \mathrm{H}_{4}$ conformers of $1,3,4,12$, and 15 monitored against the forming $\mathrm{C}_{1}-\mathrm{N}$ bond at the PBE0/DEF2-SVP/D3BJ/CPCM(CH3CN)/RIJCOSX level.

Figure S21. Correlation between NBO energy and reaction transition state energy for minimum energy ${ }^{5} \mathrm{H}_{4}$ conformer of selected glycals

Figure S22. Correlation between [4+2] to [2+2] product ratio and NBO energy gap ..................... S21

Figure S23. Transition state dynamics of ${ }^{5} \mathrm{H}_{4}$ vs ${ }^{4} \mathrm{H}_{5}$ conformers ................................................ S22

Figure S23. Quasiclassical trajectories for the ${ }^{5} \mathrm{H}_{4}$ (left) and ${ }^{4} \mathrm{H}_{5}$ (right) conformers of 3............ S22

Figure S24-S28. Comparison of correlation models.............................................................. 23

Figure S24. Correlation between experimental $-\ln \left(\mathrm{k}_{\mathrm{A}}\right)$ and the Boltzmann-weighted HOMO-LUMO gap for the ${ }^{5} \mathrm{H}_{4}$ conformers of the substrates at the PBEO/DEF2-

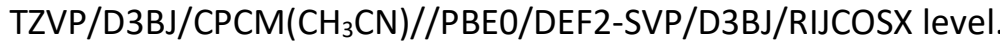

Figure S25. Correlation between experimental $-\ln \left(\mathrm{k}_{\mathrm{A}}\right)$ and the Boltzmann-weighted NBO $\pi^{*}{ }_{\mathrm{TCAl}} / \pi_{\mathrm{C} 1-}$ C2 gap uncorrected for $\pi \rightarrow \sigma^{*}$ delocalizations for the ${ }^{5} \mathrm{H}_{4}$ conformers of the substrates at the PBE0/DEF2-TZVP/D3BJ/CPCM(CH $\left.{ }_{3} \mathrm{CN}\right) / /$ PBE0/DEF2-SVP/D3BJ/RIJCOSX level

Figure S26. Correlation between experimental $-\ln \left(\mathrm{k}_{\mathrm{obs}}\right)$ and the Boltzmann-weighted HOMO-LUMO gap for all conformers of the substrates at the PBE0/DEF2-TZVP/D3BJ/CPCM(CH $\left.{ }_{3} C N\right) / / P B E 0 / D E F 2-$ SVP/D3BJ/RIJCOSX level.

Figure S28. Correlation between $-\ln \left(\mathrm{k}_{\mathrm{A}}\right)$ and the Boltzmann-weighted ${ }^{5} \mathrm{H}_{4} \mathrm{HOMO}$ energies at the PBE0/DEF2-TZVP/D3BJ/CPCM(CH ${ }_{3} \mathrm{CN}$ )//PBE0/DEF2-SVP/D3BJ/RIJCOSX level corrected for the $\pi$ bond expansion coefficients as determined from $\mathrm{CMO}$ analyses.

Supplementary Methods ..................................................................................................S28

Adjustment of experimental reaction rate for ${ }^{5} \mathrm{H}_{4}$ conformer population.....................................528

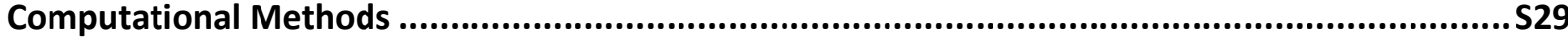

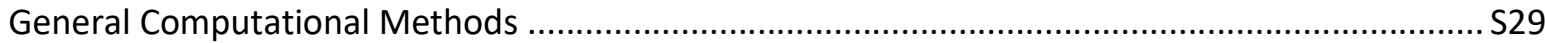

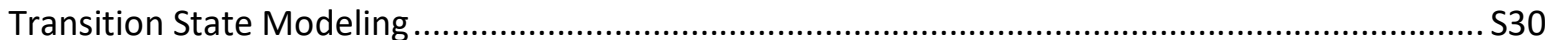

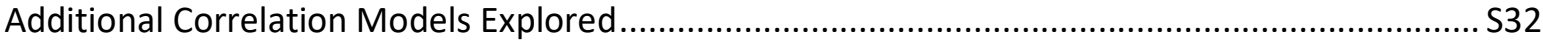

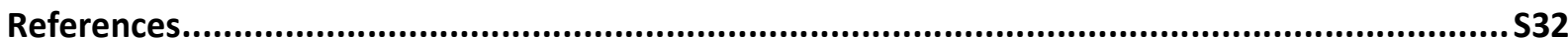

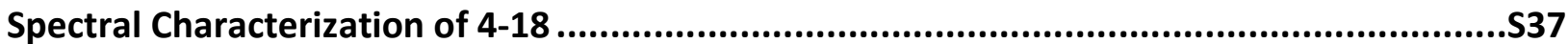

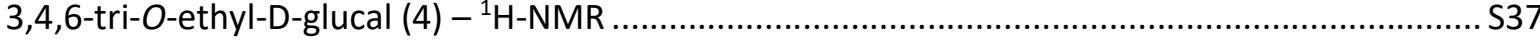

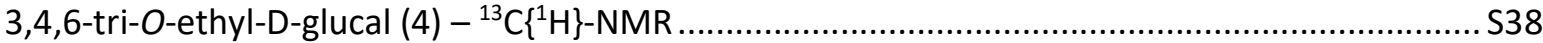

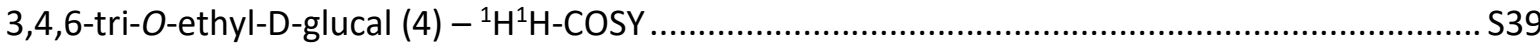

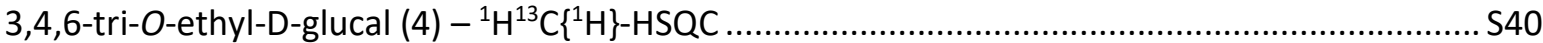

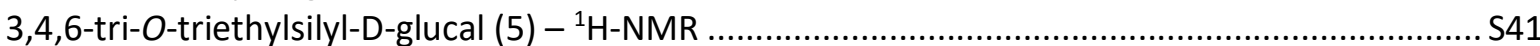

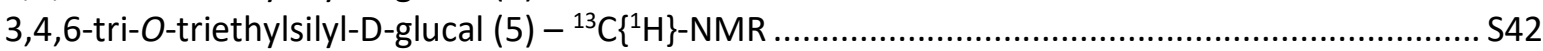

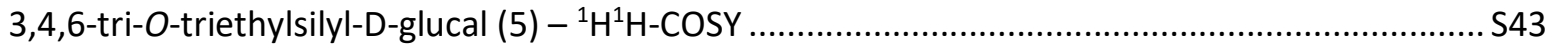

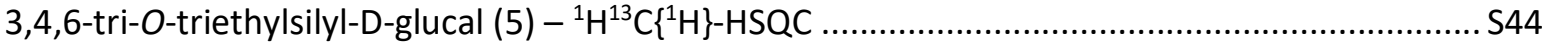

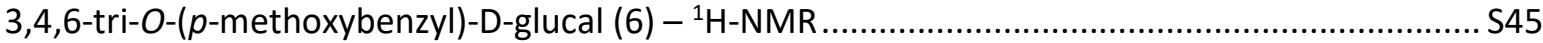




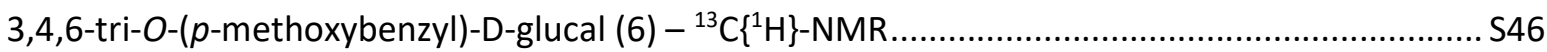

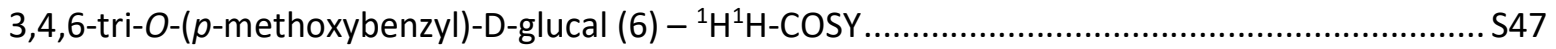

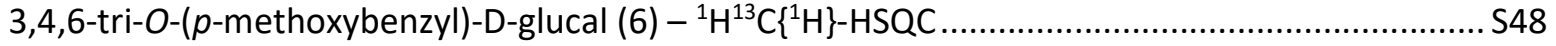

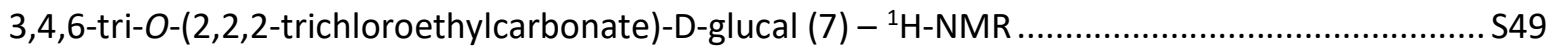

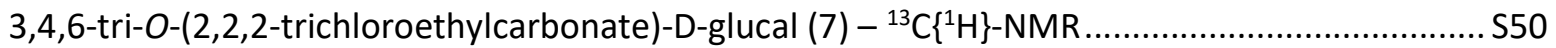

3,4,6-tri-O-(2,2,2-trichloroethylcarbonate)-D-glucal (7) - ${ }^{1} \mathrm{H}^{1} \mathrm{H}$-COSY ......................................... S51

3,4,6-tri-O-(2,2,2-trichloroethylcarbonate)-D-glucal (7) - ${ }^{1} \mathrm{H}^{13} \mathrm{C}\left\{{ }^{1} \mathrm{H}\right\}$-HSQC ................................. S52

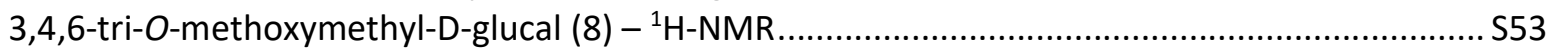

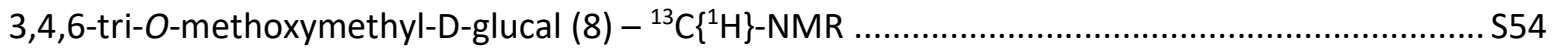

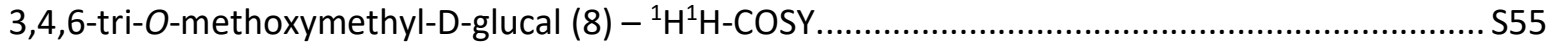

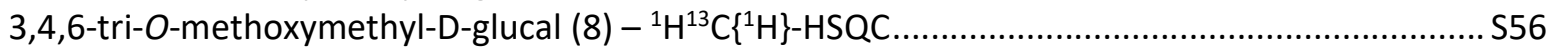

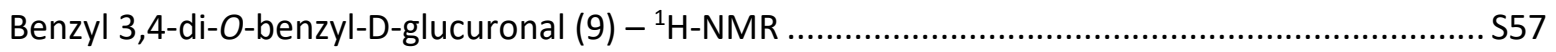

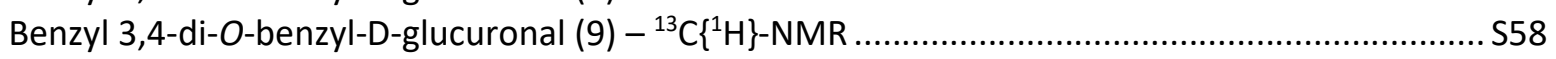

Benzyl 3,4-di-O-benzyl-D-glucuronal (9) $-{ }^{1} \mathrm{H}^{1} \mathrm{H}$-COSY ............................................................ S59

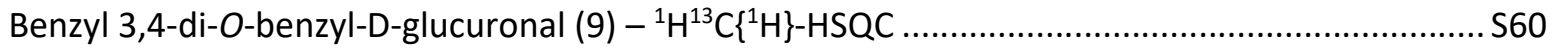

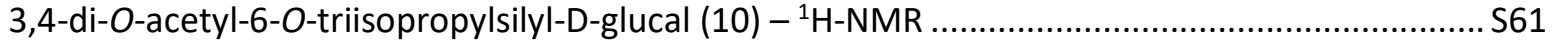

3,4-di-O-acetyl-6-O-triisopropylsilyl-D-glucal (10) $-{ }^{13} \mathrm{C}\left\{{ }^{1} \mathrm{H}\right\}$-NMR ............................................. 62

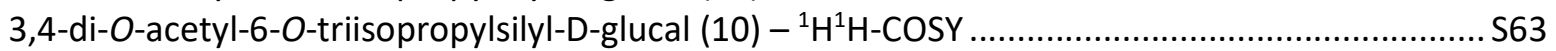

3,4-di-O-acetyl-6-O-triisopropylsilyl-D-glucal (10) $-{ }^{1} \mathrm{H}^{13} \mathrm{C}\left\{{ }^{1} \mathrm{H}\right\}$-HSQC ........................................... S64

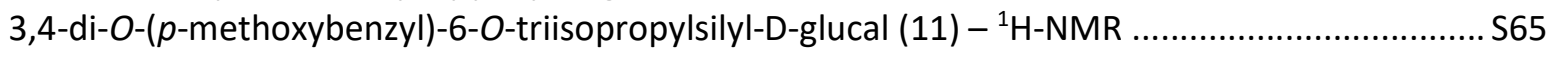

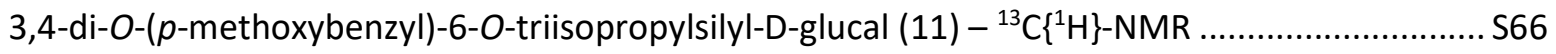

3,4-di-O-(p-methoxybenzyl)-6-O-triisopropylsilyl-D-glucal (11) $-{ }^{1} \mathrm{H}^{1} \mathrm{H}$-COSY .............................. 667

3,4-di-O-(p-methoxybenzyl)-6-O-triisopropylsilyl-D-glucal (11) $-{ }^{1} \mathrm{H}^{13} \mathrm{C}\left\{{ }^{1} \mathrm{H}\right\}$-HSQC........................S68

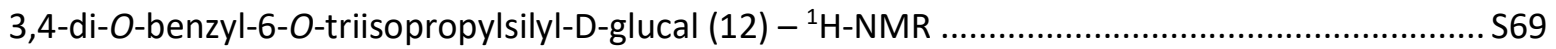

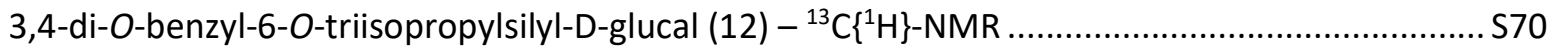

3,4-di-O-benzyl-6-O-triisopropylsilyl-D-glucal (12) - ${ }^{1} \mathrm{H}^{1} \mathrm{H}$-COSY ................................................. S71

3,4-di-O-benzyl-6-O-triisopropylsilyl-D-glucal (12) - ${ }^{1} \mathrm{H}^{13} \mathrm{C}\left\{{ }^{1} \mathrm{H}\right\}$-HSQC ........................................... 572

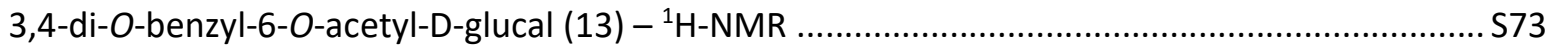

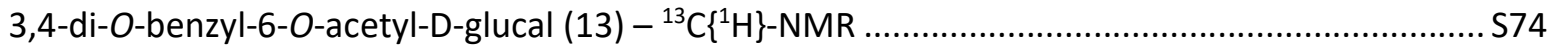

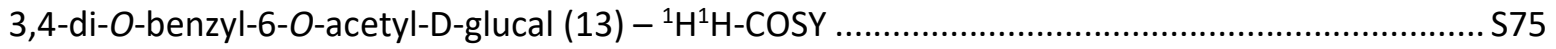

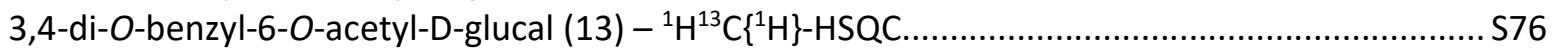

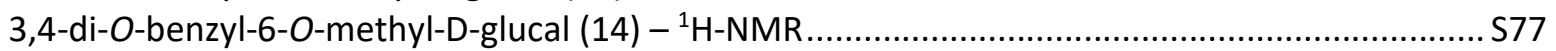

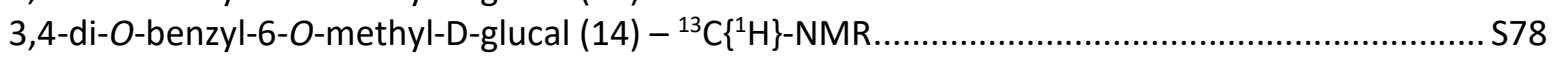

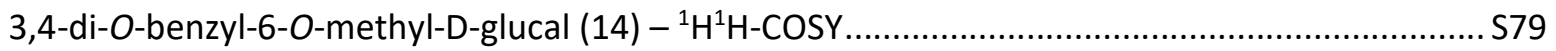

3,4-di-O-benzyl-6-O-methyl-D-glucal (14) $-{ }^{1} \mathrm{H}^{13} \mathrm{C}\left\{{ }^{1} \mathrm{H}\right\}$-HSQC ..................................................... S80

3,4-di-O-benzyl-6-O-(2,2,2-trichloroethylcarbonate)-D-glucal (15) - ${ }^{1} \mathrm{H}-\mathrm{NMR}$............................. S81

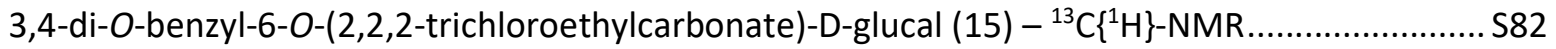

3,4-di-O-benzyl-6-O-(2,2,2-trichloroethylcarbonate)-D-glucal (15) $-{ }^{1} \mathrm{H}^{1} \mathrm{H}$-COSY ......................... 883

3,4-di-O-benzyl-6-O-(2,2,2-trichloroethylcarbonate)-D-glucal (15) - ${ }^{1} \mathrm{H}^{13} \mathrm{C}\left\{{ }^{1} \mathrm{H}\right\}$-HSQC .................. S84

3,4-di-O-benzyl-6-O-trityl-D-glucal (16) - ${ }^{1} \mathrm{H}$-NMR ................................................................. 885

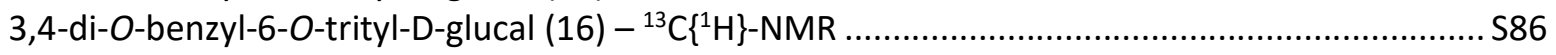

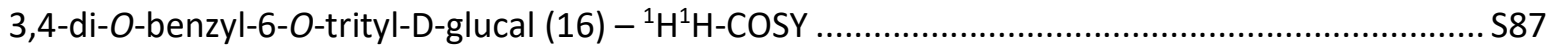

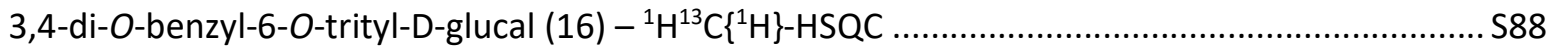

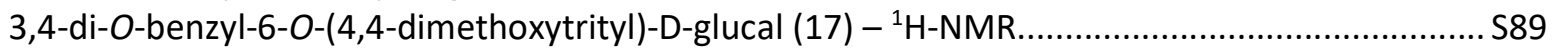

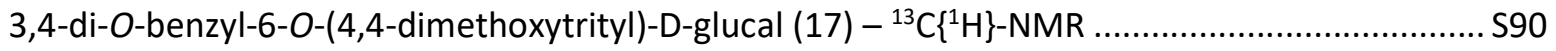

3,4-di-O-benzyl-6-O-(4,4-dimethoxytrityl)-D-glucal (17) - ${ }^{1} \mathrm{H}^{1} \mathrm{H}$-COSY ......................................... S91

3,4-di-O-benzyl-6-O-(4,4-dimethoxytrityl)-D-glucal (17) - ${ }^{1} \mathrm{H}^{13} \mathrm{C}\left\{{ }^{1} \mathrm{H}\right\}-\mathrm{HSQC}$.................................. S92 
Spectra of Cycloaddition Reaction Mixtures .............................................................S93

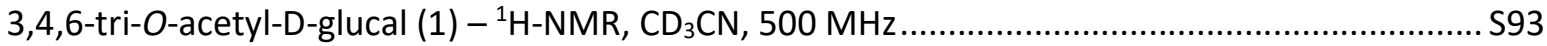

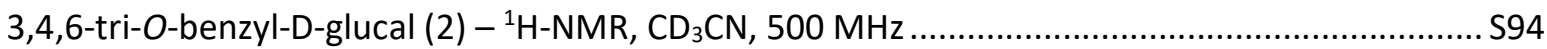

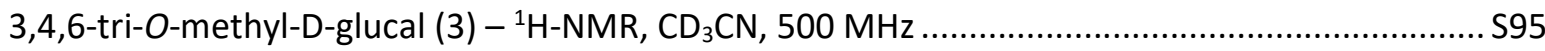

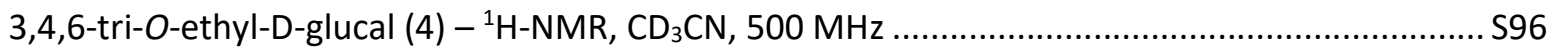

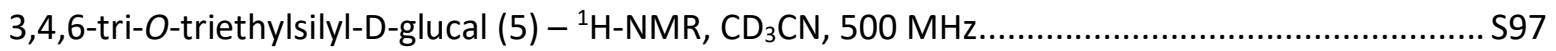

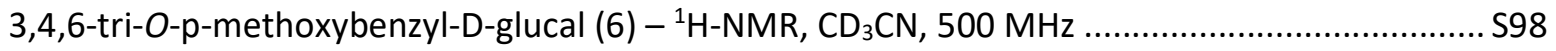

3,4,6-tri-O-2,2,2-trichloroethylcarbonate-D-glucal (7) - ${ }^{1} \mathrm{H}-\mathrm{NMR}, \mathrm{CD}_{3} \mathrm{CN}, 500 \mathrm{MHz} \ldots \ldots \ldots \ldots \ldots \ldots \ldots . . . . . . . .599$

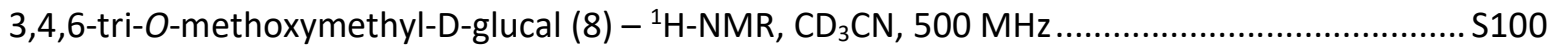

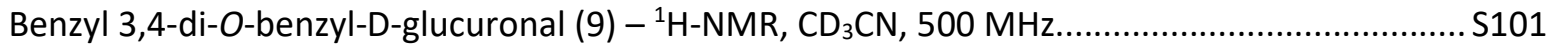

3,4-di-O-acetyl-6-O-triisopropylsilyl-D-glucal (10) - ${ }^{1} \mathrm{H}-\mathrm{NMR}, \mathrm{CD}_{3} \mathrm{CN}, 500 \mathrm{MHz}$.......................... 102

3,4-di-O-p-methoxybenzyl-6-O-triisopropylsilyl-D-glucal (11) - ${ }^{1} \mathrm{H}-\mathrm{NMR}, \mathrm{CD}_{3} \mathrm{CN}, 500 \mathrm{MHz} \ldots \ldots . . . \mathrm{S} 103$

3,4-di-O-benzyl-6-O-triisopropylsilyl-D-glucal (12) - ${ }^{1} \mathrm{H}-\mathrm{NMR}, \mathrm{CD}_{3} \mathrm{CN}, 500 \mathrm{MHz} \ldots \ldots \ldots \ldots \ldots \ldots \ldots . . . . . . . . . . . . . .104$

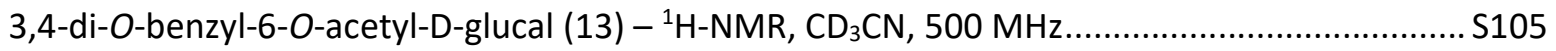

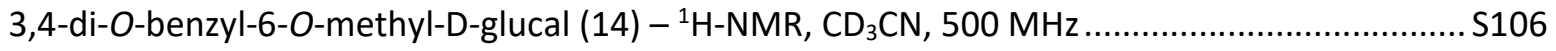

3,4-di-O-benzyl-6-O-2,2,2-trichloroethylcarbonate-D-glucal (14) - ${ }^{1} \mathrm{H}-\mathrm{NMR}, \mathrm{CD}_{3} \mathrm{CN}, 500 \mathrm{MHz} . . . \mathrm{S} 107$

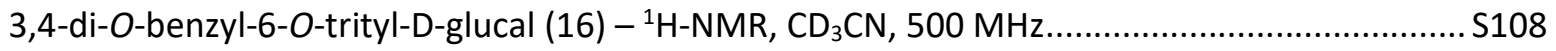

3,4-di-O-benzyl-6-O-4,4-dimethoxytrityl-D-glucal (17) - ${ }^{1} \mathrm{H}-\mathrm{NMR}, \mathrm{CD}_{3} \mathrm{CN}, 500 \mathrm{MHz}$................... S109

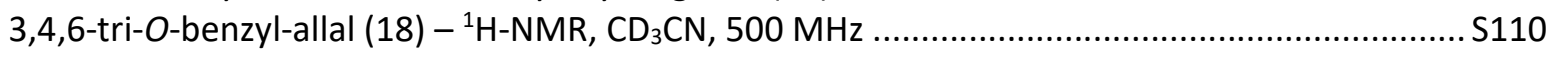

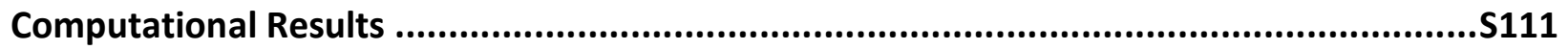

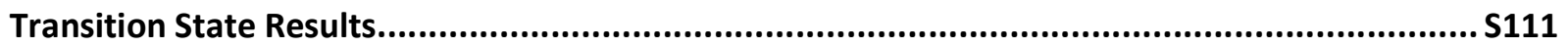

Table SCX1. Energetics of cycloaddition between TCAI and various substrates at the PBE0/DEF2-

TZVP/CPCM(CH $\left.{ }_{3} \mathrm{CH}\right) / D 3 B J / / P B E 0 / D E F 2-S V P / C P C M\left(\mathrm{CH}_{3} \mathrm{CN}\right) / D 3 B J$ level. ................................... S111

Energetics for Sampled Conformations ............................................................................ S111

Table SCY1. Energetics for 1 at the PBE0/D3BJ/CPCM(CH $\left.\mathrm{CN}_{3}\right) / /$ PBE0/D3BJ/DEF2-SVP/RIJCOSX level.

Relative energies in $\mathrm{kcal} / \mathrm{mol}$....................................................................................... 111

Table SCY2. Energetics for 2 at the PBE0/D3BJ/CPCM(CH $\left.{ }_{3} \mathrm{CN}\right) / /$ PBEO/D3BJ/DEF2-SVP/RIJCOSX level.

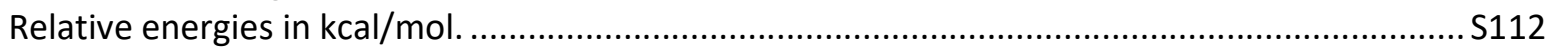

Table SCY3. Energetics for 3 at the PBE0/D3BJ/CPCM(CH $\left.\mathrm{CH}_{3} \mathrm{CN}\right) / /$ PBE0/D3BJ/DEF2-SVP/RIJCOSX level.

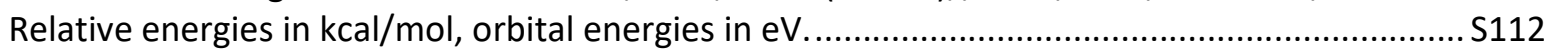

Table SCY4. Energetics for 4 at the PBE0/D3BJ/CPCM(CH $\left.{ }_{3} \mathrm{CN}\right) / /$ PBE0/D3BJ/DEF2-SVP/RIJCOSX level.

Relative energies in $\mathrm{kcal} / \mathrm{mol}$, orbital energies in $\mathrm{eV}$.......................................................... 113

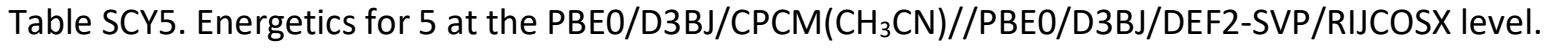

Relative energies in $\mathrm{kcal} / \mathrm{mol}$, orbital energies in $\mathrm{eV}$............................................................. S113

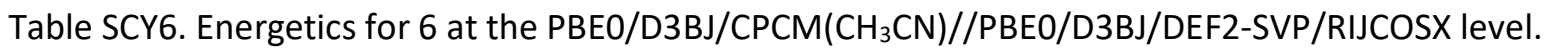

Relative energies in $\mathrm{kcal} / \mathrm{mol}$, orbital energies in $\mathrm{eV}$......................................................... 1115

Table SCY7. Energetics for 7 at the PBE0/D3BJ/CPCM(CH $\left.{ }_{3} \mathrm{CN}\right) / /$ PBE0/D3BJ/DEF2-SVP/RIJCOSX level.

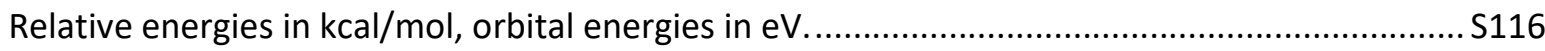

Table SCY8. Energetics for 9 at the PBE0/D3BJ/CPCM(CH $\left.{ }_{3} \mathrm{CN}\right) / /$ PBEO/D3BJ/DEF2-SVP/RIJCOSX level.

Relative energies in $\mathrm{kcal} / \mathrm{mol}$, orbital energies in $\mathrm{eV}$............................................................. S118

Table SCY9. Energetics for 10 at the PBE0/D3BJ/CPCM(CH $\left.{ }_{3} \mathrm{CN}\right) / / P B E 0 / D 3 B J / D E F 2-S V P / R I J C O S X$

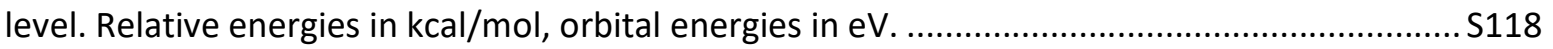

Table SCY10. Energetics for 11 at the PBE0/D3BJ/CPCM(CH $\mathrm{CHN}_{3}$ //PBE0/D3BJ/DEF2-SVP/RIJCOSX

level. Relative energies in $\mathrm{kcal} / \mathrm{mol}$, orbital energies in $\mathrm{eV}$. ................................................. S119

Table SCY11. Energetics for 12 at the PBE0/D3BJ/CPCM(CH $\mathrm{CHN}_{3}$ //PBE0/D3BJ/DEF2-SVP/RIJCOSX

level. Relative energies in $\mathrm{kcal} / \mathrm{mol}$, orbital energies in $\mathrm{eV}$.

S120 
Table SCY12. Energetics for 13 at the PBE0/D3BJ/CPCM(CH 3 CN)//PBE0/D3BJ/DEF2-SVP/RIJCOSX

level. Relative energies in $\mathrm{kcal} / \mathrm{mol}$, orbital energies in $\mathrm{eV}$.

Table SCY13. Energetics for 14 at the PBE0/D3BJ/CPCM(CH $\left.\mathrm{CH}_{3} \mathrm{CN}\right) / / \mathrm{PBE0} / \mathrm{D} 3 \mathrm{BJ} / \mathrm{DEF} 2-\mathrm{SVP} / \mathrm{RIJCOSX}$

level. Relative energies in $\mathrm{kcal} / \mathrm{mol}$, orbital energies in $\mathrm{eV}$. S123

Table SCY14. Energetics for 15 at the PBE0/D3BJ/CPCM(CH $\left.{ }_{3} \mathrm{CN}\right) / / P B E 0 / D 3 B J / D E F 2-S V P / R I J C O S X$

level. Relative energies in $\mathrm{kcal} / \mathrm{mol}$, orbital energies in $\mathrm{eV}$.

Equilibrium Structures of ${ }^{5} \mathrm{H}_{4}$ and ${ }^{4} \mathrm{H}_{5}$ Global Minima.

Table SCZ1. Cartesian coordinates for the global minimum ${ }^{5} \mathrm{H}_{4}$ conformer of 1 at the PBE0/DEF2TZVP/D3BJ/CPCM $\left(\mathrm{CH}_{3} \mathrm{CN}\right) / \mathrm{RIJCOSX}$ level...

Table SCZ2. Cartesian coordinates for the global minimum ${ }^{4} \mathrm{H}_{5}$ conformer of 1 at the PBEO/DEF2-

TZVP/D3BJ/CPCM( $\left.\mathrm{CH}_{3} \mathrm{CN}\right) / \mathrm{RIJCOSX}$ level.

Table SCZ3. Cartesian coordinates for the global minimum ${ }^{5} \mathrm{H}_{4}$ conformer of 2 at the PBEO/DEF2-

TZVP/D3BJ/CPCM( $\left.\mathrm{CH}_{3} \mathrm{CN}\right) / \mathrm{RIJCOSX}$ level.

Table SCZ4. Cartesian coordinates for the global minimum ${ }^{4} \mathrm{H}_{5}$ conformer of 2 at the PBE0/DEF2-

TZVP/D3BJ/CPCM $\left(\mathrm{CH}_{3} \mathrm{CN}\right) / \mathrm{RIJCOSX}$ level...

Table SCZ5. Cartesian coordinates for the global minimum ${ }^{5} \mathrm{H}_{4}$ conformer of 3 at the PBEO/DEF2-

TZVP/D3BJ/CPCM( $\left.\mathrm{CH}_{3} \mathrm{CN}\right) / \mathrm{RIJCOSX}$ level.

Table SCZ6. Cartesian coordinates for the global minimum ${ }^{4} \mathrm{H}_{5}$ conformer of 3 at the PBEO/DEF2-

TZVP/D3BJ/CPCM( $\left.\mathrm{CH}_{3} \mathrm{CN}\right) / \mathrm{RIJCOSX}$ level.

Table SCZ7. Cartesian coordinates for the global minimum ${ }^{5} \mathrm{H}_{4}$ conformer of 4 at the PBEO/DEF2-

TZVP/D3BJ/CPCM( $\left.\mathrm{CH}_{3} \mathrm{CN}\right) / \mathrm{RIJCOSX}$ level.

Table SCZ8. Cartesian coordinates for the global minimum ${ }^{4} \mathrm{H}_{5}$ conformer of 4 at the PBE0/DEF2-

TZVP/D3BJ/CPCM( $\left.\mathrm{CH}_{3} \mathrm{CN}\right) / \mathrm{RIJCOSX}$ level.

Table SCZ9. Cartesian coordinates for the global minimum ${ }^{5} \mathrm{H}_{4}$ conformer of 5 at the PBE0/DEF2-

TZVP/D3BJ/CPCM( $\left.\mathrm{CH}_{3} \mathrm{CN}\right) / \mathrm{RIJCOSX}$ level.

Table SCZ10. Cartesian coordinates for the global minimum ${ }^{5} \mathrm{H}_{4}$ conformer of 6 at the PBE0/DEF2-

TZVP/D3BJ/CPCM( $\left.\mathrm{CH}_{3} \mathrm{CN}\right) / \mathrm{RIJCOSX}$ level.

Table SCZ11. Cartesian coordinates for the global minimum ${ }^{4} \mathrm{H}_{5}$ conformer of 6 at the PBE0/DEF2-

TZVP/D3BJ/CPCM $\left(\mathrm{CH}_{3} \mathrm{CN}\right) / \mathrm{RIJCOSX}$ level...

Table SCZ12. Cartesian coordinates for the global minimum ${ }^{5} \mathrm{H}_{4}$ conformer of 7 at the PBEO/DEF2-

TZVP/D3BJ/CPCM( $\left.\mathrm{CH}_{3} \mathrm{CN}\right) / \mathrm{RIJCOSX}$ level.

Table SCZ13. Cartesian coordinates for the global minimum ${ }^{4} \mathrm{H}_{5}$ conformer of 7 at the PBE0/DEF2-

TZVP/D3BJ/CPCM( $\left.\mathrm{CH}_{3} \mathrm{CN}\right) / \mathrm{RIJCOSX}$ level..

$\mathrm{S} 135$

Table SCZ14. Cartesian coordinates for the global minimum ${ }^{5} \mathrm{H}_{4}$ conformer of 9 at the PBE0/DEF2-

TZVP/D3BJ/CPCM(CH $\left.{ }_{3} \mathrm{CN}\right) / \mathrm{RIJCOSX}$ level.

S136

Table SCZ15. Cartesian coordinates for the global minimum ${ }^{5} \mathrm{H}_{4}$ conformer of 10 at the PBEO/DEF2TZVP/D3BJ/CPCM $\left(\mathrm{CH}_{3} \mathrm{CN}\right) / \mathrm{RIJCOSX}$ level.

S137

Table SCZ16. Cartesian coordinates for the global minimum ${ }^{5} \mathrm{H}_{4}$ conformer of 11 at the PBE0/DEF2-

TZVP/D3BJ/CPCM $\left(\mathrm{CH}_{3} \mathrm{CN}\right) / \mathrm{RIJCOSX}$ level.

$\mathrm{S} 138$

Table SCZ17. Cartesian coordinates for the global minimum ${ }^{4} \mathrm{H}_{5}$ conformer of 11 at the PBE0/DEF2-

TZVP/D3BJ/CPCM(CH $\left.{ }_{3} \mathrm{CN}\right) / \mathrm{RIJCOSX}$ level.

S139

Table SCZ18. Cartesian coordinates for the global minimum ${ }^{5} \mathrm{H}_{4}$ conformer of 12 at the PBEO/DEF2-

TZVP/D3BJ/CPCM(CH $\left.{ }_{3} \mathrm{CN}\right) / \mathrm{RIJCOSX}$ level.

S141

Table SCZ19. Cartesian coordinates for the global minimum ${ }^{4} \mathrm{H}_{5}$ conformer of 12 at the PBE0/DEF2-

TZVP/D3BJ/CPCM $\left(\mathrm{CH}_{3} \mathrm{CN}\right) / \mathrm{RIJCOSX}$ level

Table SCZ20. Cartesian coordinates for the global minimum ${ }^{5} \mathrm{H}_{4}$ conformer of 13 at the PBEO/DEF2-

TZVP/D3BJ/CPCM $\left(\mathrm{CH}_{3} \mathrm{CN}\right) / \mathrm{RIJCOSX}$ level 
Table SCZ21. Cartesian coordinates for the global minimum ${ }^{4} \mathrm{H}_{5}$ conformer of 13 at the PBEO/DEF2TZVP/D3BJ/CPCM $\left(\mathrm{CH}_{3} \mathrm{CN}\right) / \mathrm{RIJCOSX}$ level......

Table SCZ22. Cartesian coordinates for the global minimum ${ }^{5} \mathrm{H}_{4}$ conformer of 14 at the PBEO/DEF2TZVP/D3BJ/CPCM $\left(\mathrm{CH}_{3} \mathrm{CN}\right) / \mathrm{RIJCOSX}$ level. S145

Table SCZ23. Cartesian coordinates for the global minimum ${ }^{4} \mathrm{H}_{5}$ conformer of 14 at the PBE0/DEF2-

TZVP/D3BJ/CPCM $\left(\mathrm{CH}_{3} \mathrm{CN}\right) / \mathrm{RIJCOSX}$ level... S146

Table SCZ24. Cartesian coordinates for the global minimum ${ }^{5} \mathrm{H}_{4}$ conformer of 15 at the PBEO/DEF2TZVP/D3BJ/CPCM( $\left.\mathrm{CH}_{3} \mathrm{CN}\right) / \mathrm{RIJCOSX}$ level...

Table SCZ25. Cartesian coordinates for the global minimum ${ }^{4} \mathrm{H}_{5}$ conformer of 15 at the PBEO/DEF2TZVP/D3BJ/CPCM( $\left.\mathrm{CH}_{3} \mathrm{CN}\right) / \mathrm{RIJCOSX}$ level. S148

Table SCZ26. Cartesian coordinates for cycloaddition transition state of the ${ }^{5} \mathrm{H}_{4}$ conformer of 1 with TCAl at the PBEO/DEF2-SVP/D3BJ/CPCM $\left(\mathrm{CH}_{3} \mathrm{CN}\right) / \mathrm{RIJCOSX}$ level. S149

Table SCZ27. Cartesian coordinates for cycloaddition transition state of the ${ }^{4} \mathrm{H}_{5}$ conformer of 1 with TCAI at the PBE0/DEF2-SVP/D3BJ/CPCM $\left(\mathrm{CH}_{3} \mathrm{CN}\right) / \mathrm{RIJCOSX}$ level. S150 Table SCZ28. Cartesian coordinates for cycloaddition transition state of the ${ }^{5} \mathrm{H}_{4}$ conformer of 3 with TCAl at the PBE0/DEF2-SVP/D3BJ/CPCM $\left(\mathrm{CH}_{3} \mathrm{CN}\right) / \mathrm{RIJCOSX}$ level. S150 Table SCZ29. Cartesian coordinates for cycloaddition transition state of the ${ }^{4} \mathrm{H}_{5}$ conformer of 3 with TCAl at the PBEO/DEF2-SVP/D3BJ/CPCM $\left(\mathrm{CH}_{3} \mathrm{CN}\right) / \mathrm{RIJCOSX}$ level. S151 Table SCZ30. Cartesian coordinates for cycloaddition transition state of the ${ }^{5} \mathrm{H}_{4}$ conformer of 4 with TCAl at the PBE0/DEF2-SVP/D3BJ/CPCM $\left(\mathrm{CH}_{3} \mathrm{CN}\right) / \mathrm{RIJCOSX}$ level

Table SCZ31. Cartesian coordinates for cycloaddition transition state of the ${ }^{4} \mathrm{H}_{5}$ conformer of 4 with

TCAI at the PBE0/DEF2-SVP/D3BJ/CPCM(CH $\left.{ }_{3} \mathrm{CN}\right) / \mathrm{RIJCOSX}$ level. S153

Table SCZ32. Cartesian coordinates for cycloaddition transition state of the ${ }^{5} \mathrm{H}_{4}$ conformer of 12 with TCAl at the PBE0/DEF2-SVP/D3BJ/CPCM $\left(\mathrm{CH}_{3} \mathrm{CN}\right) / \mathrm{RIJCOSX}$ level. S153

Table SCZ33. Cartesian coordinates for cycloaddition transition state of the ${ }^{4} \mathrm{H}_{5}$ conformer of 12 with TCAl at the PBE0/DEF2-SVP/D3BJ/CPCM $\left(\mathrm{CH}_{3} \mathrm{CN}\right) / \mathrm{RIJCOSX}$ level. S155

Table SCZ34. Cartesian coordinates for cycloaddition transition state of the ${ }^{5} \mathrm{H}_{4}$ conformer of 15 with TCAl at the PBEO/DEF2-SVP/D3BJ/CPCM( $\left.\mathrm{CH}_{3} \mathrm{CN}\right) / \mathrm{RIJCOSX}$ level. S156 Table SCZ35. Cartesian coordinates for cycloaddition transition state of the ${ }^{4} \mathrm{H}_{5}$ conformer of 15 with TCAI at the PBE0/DEF2-SVP/D3BJ/CPCM $\left(\mathrm{CH}_{3} \mathrm{CN}\right) / \mathrm{RIJCOSX}$ level. 


\section{Supplementary Figures}

Figure S1 - ${ }^{1} \mathrm{H}^{13} \mathrm{C}-\mathrm{HSQC}$ and ${ }^{1} \mathrm{H}^{13} \mathrm{C}-\mathrm{HMBC}$ analysis of Cycloaddition Mixture
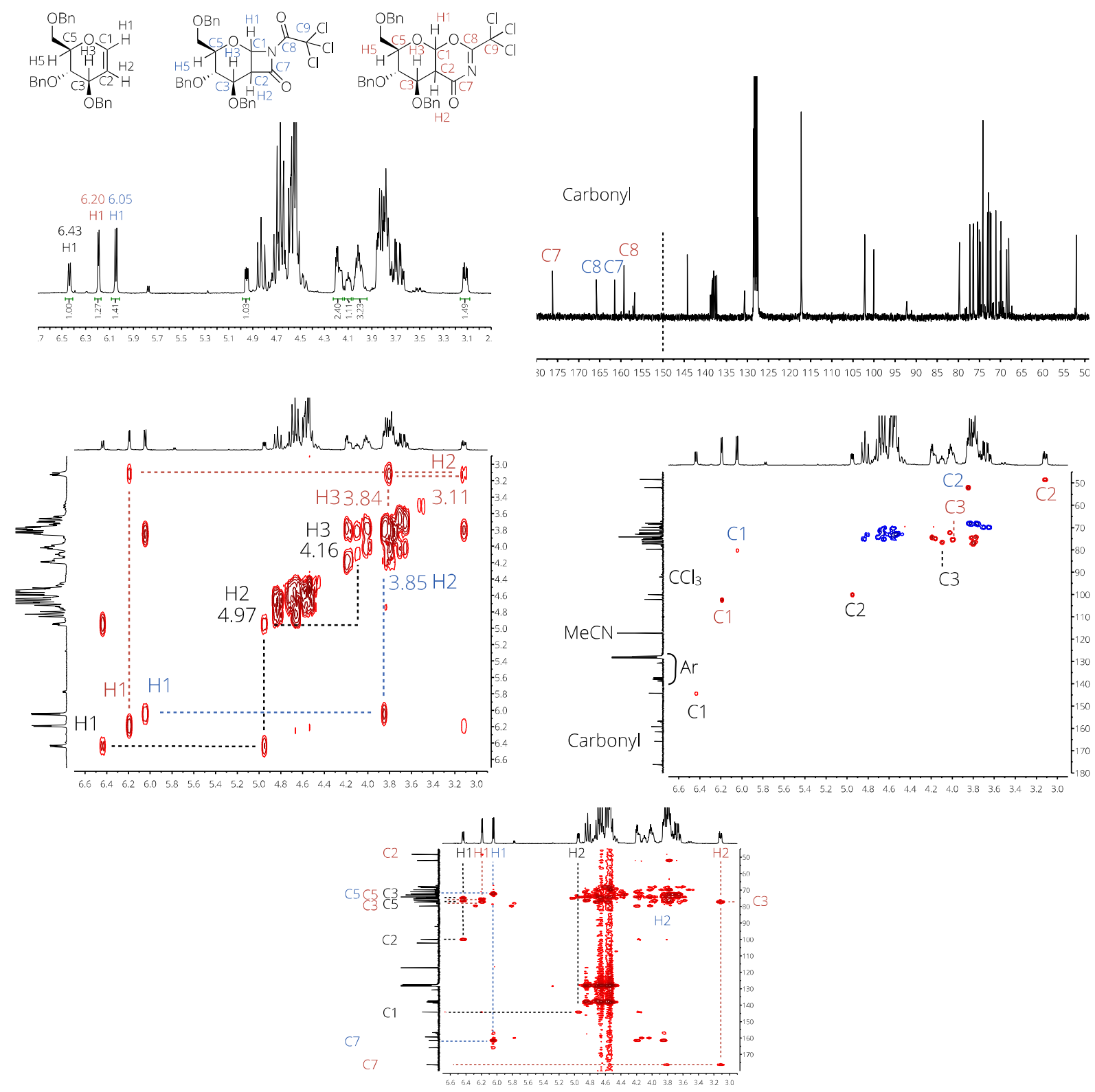

Figure S1. Spectra of cycloaddition reaction mixture and peak assignment using ${ }^{1} \mathrm{H}-\mathrm{NMR}$ (top left), ${ }^{13} \mathrm{C}$-NMR (top right), ${ }^{1} \mathrm{H}^{1} \mathrm{H}$-COSY (middle left), ${ }^{1} \mathrm{H}^{13} \mathrm{C}$-HSQC (middle right), and ${ }^{1} \mathrm{H}^{13} \mathrm{C}$ $\mathrm{HMBC}$ (bottom) all in $\mathrm{CD}_{3} \mathrm{CN}(500 \mathrm{MHz})$. Peak assignments were made by first identifying chemical shift with ${ }^{1} \mathrm{H}^{13} \mathrm{C}-\mathrm{HSQC}$, then assessing connectivity via ${ }^{1} \mathrm{H}^{1} \mathrm{H}-\mathrm{COSY}$ and ${ }^{1} \mathrm{H}^{13} \mathrm{C}$ $\mathrm{HMBC}$, followed by confirmation by ${ }^{1} \mathrm{H}-\mathrm{NMR}$ and ${ }^{13} \mathrm{C}-\mathrm{NMR}$. 
Figure S2 - Reaction progress of substrates 1-18

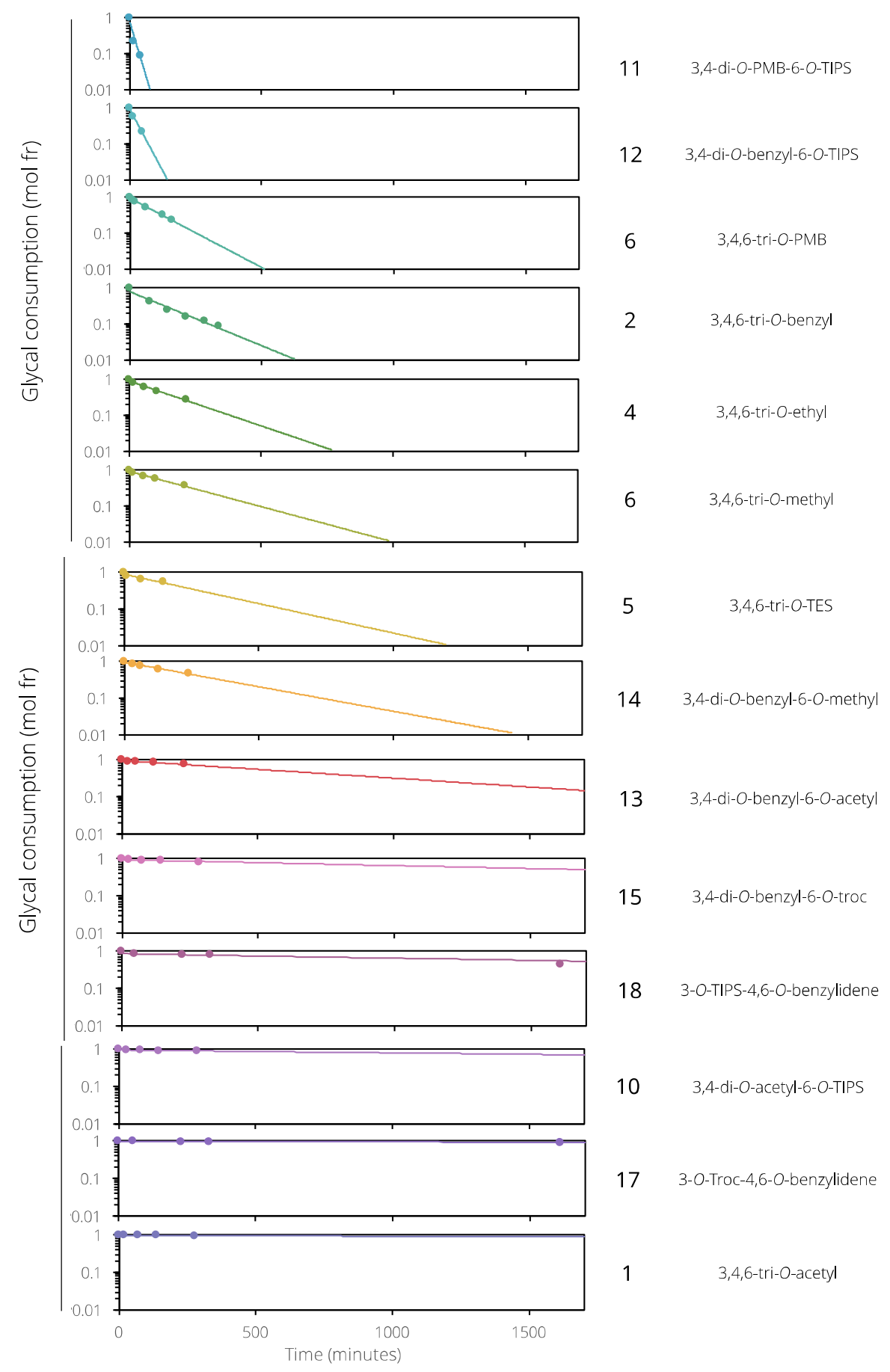

Figure S2. Time course of logarithm of glycal (indicated) percentage within the cycloaddition reaction mixture over the first 1500 minutes as measured by ${ }^{1} \mathrm{H}-\mathrm{NMR}$. Reactions are ordered from largest to slowest reaction rate. The slope of the included linear fit of the data was used to obtain the reaction rate. 
Figure S3-S16. Lowest energy ${ }^{5} \mathrm{H}_{4}$ conformers
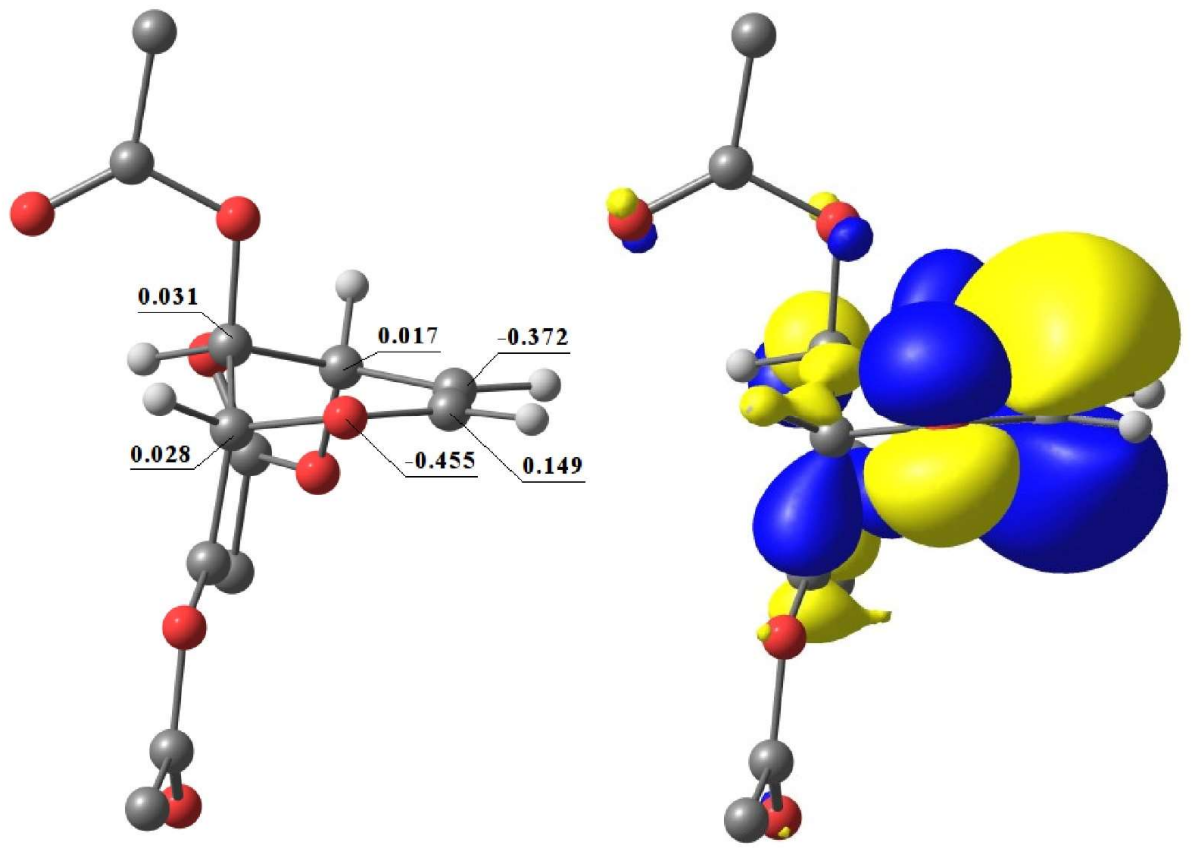

Figure S3. Lowest energy ${ }^{5} \mathrm{H}_{4}$ conformer [PBE0/DEF2-TZVP/SMD(ACN)] of 1 with ring atoms labeled with natural charges. HOMO isosurface shown on the right.
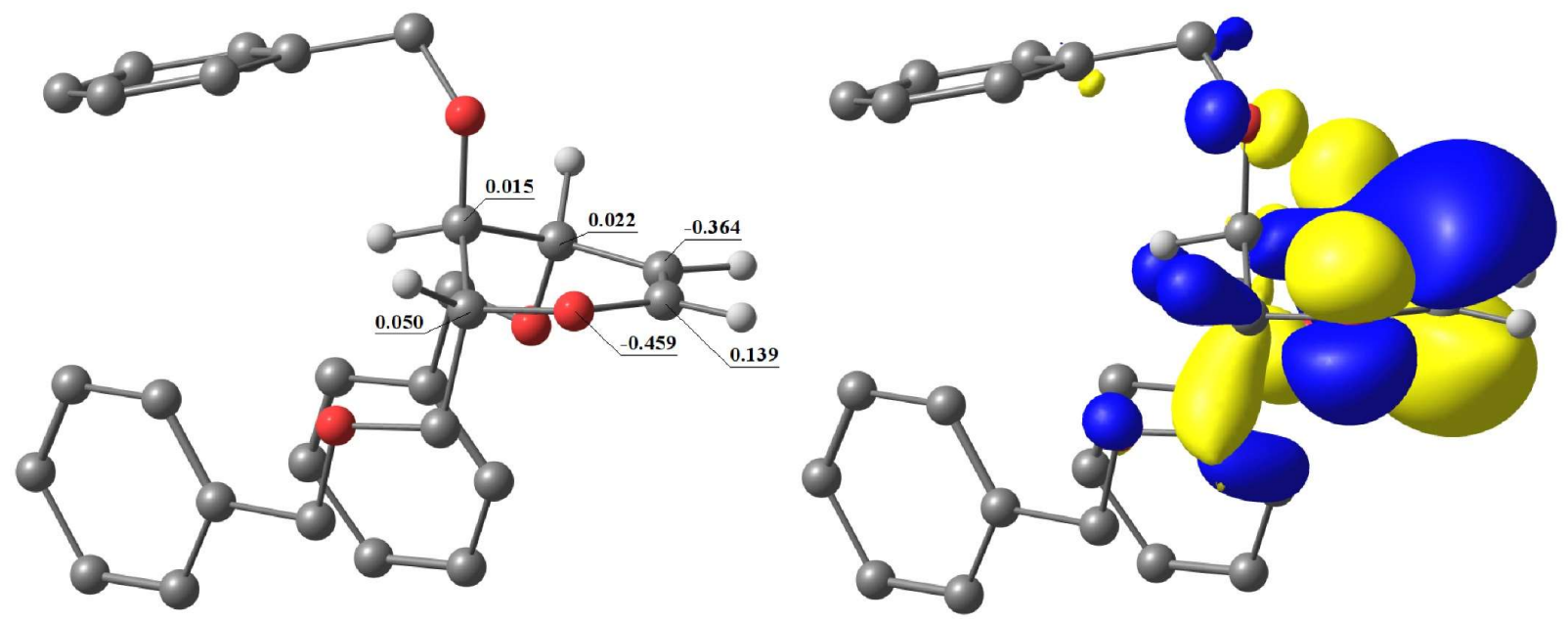

Figure S4. Lowest energy ${ }^{5} \mathrm{H}_{4}$ conformer [PBE0/DEF2-TZVP/SMD(ACN)] of 2 with ring atoms labeled with natural charges. HOMO isosurface shown on the right. 

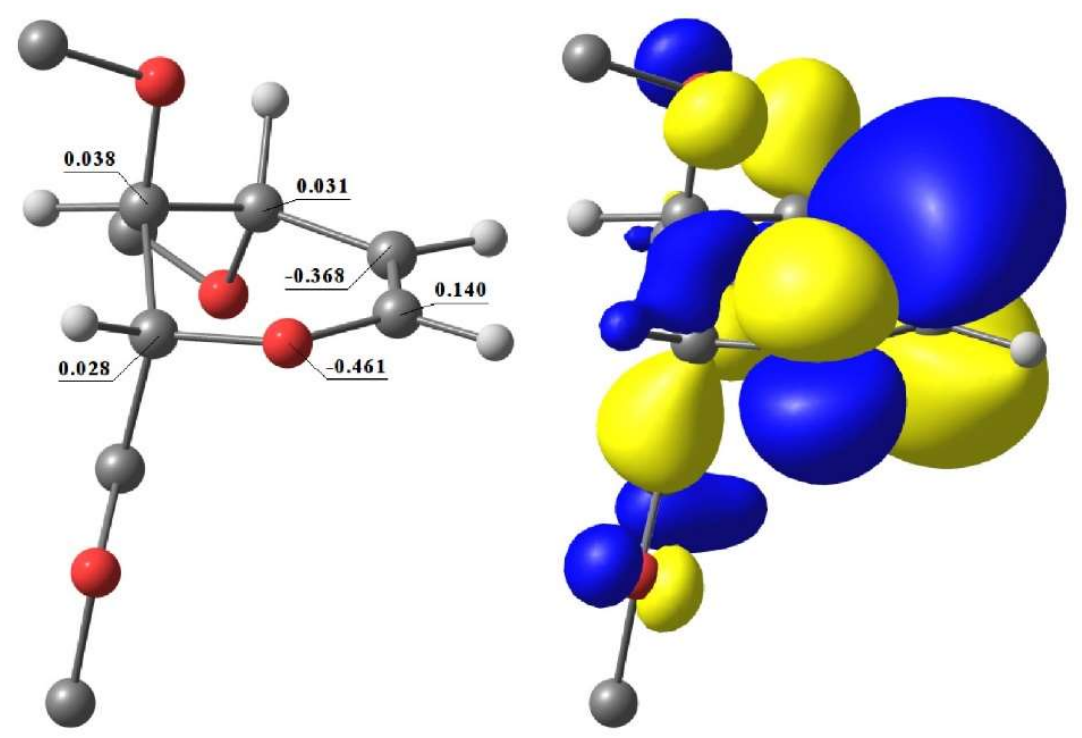

Figure S5. Lowest energy ${ }^{5} \mathrm{H}_{4}$ conformer [PBE0/DEF2-TZVP/SMD(ACN)] of 3 with ring atoms labeled with natural charges. HOMO isosurface shown on the right.
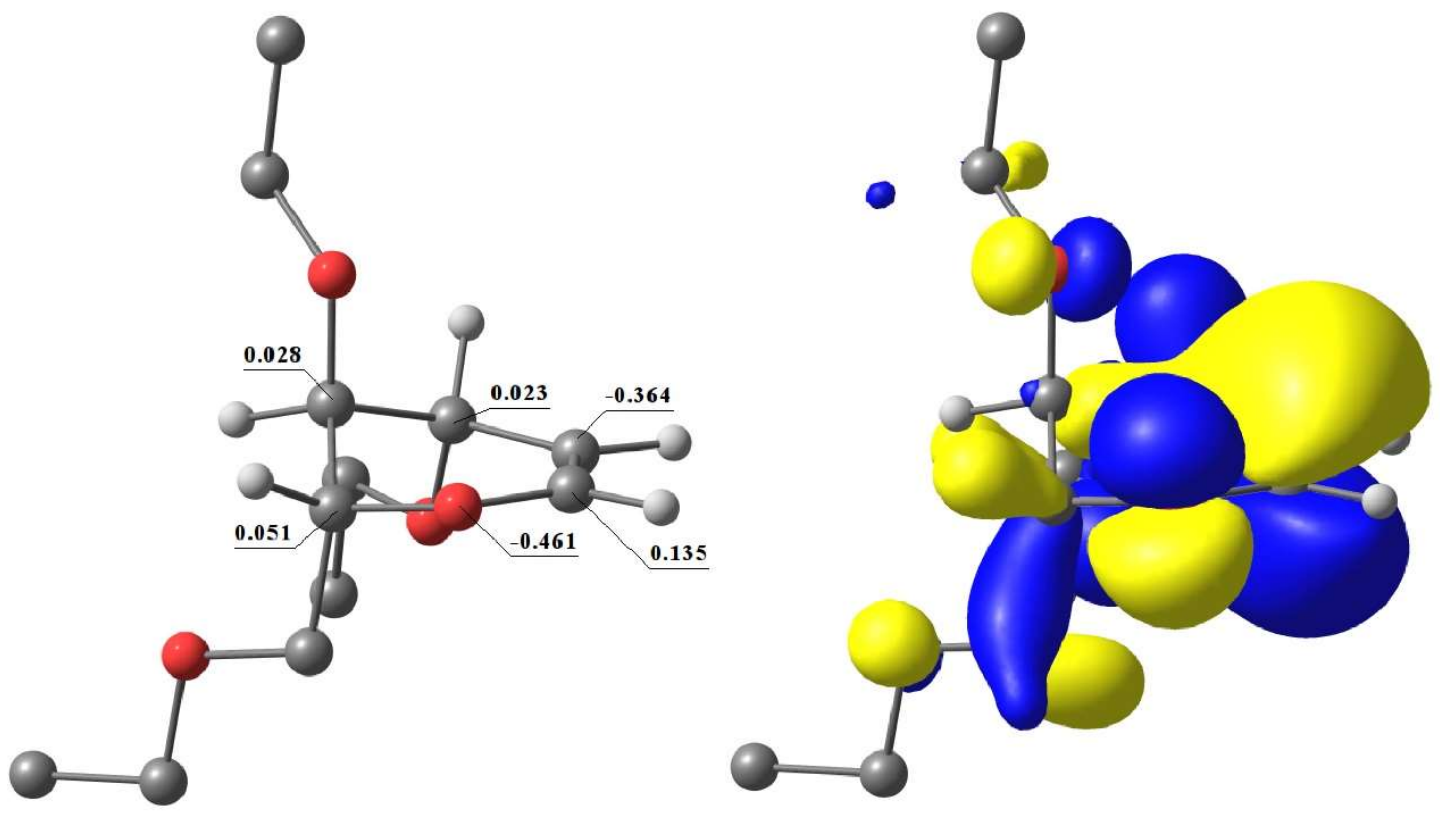

Figure S6. Lowest energy ${ }^{5} \mathrm{H}_{4}$ conformer [PBE0/DEF2-TZVP/SMD(ACN)] of 4 with ring atoms labeled with natural charges. HOMO isosurface shown on the right. 

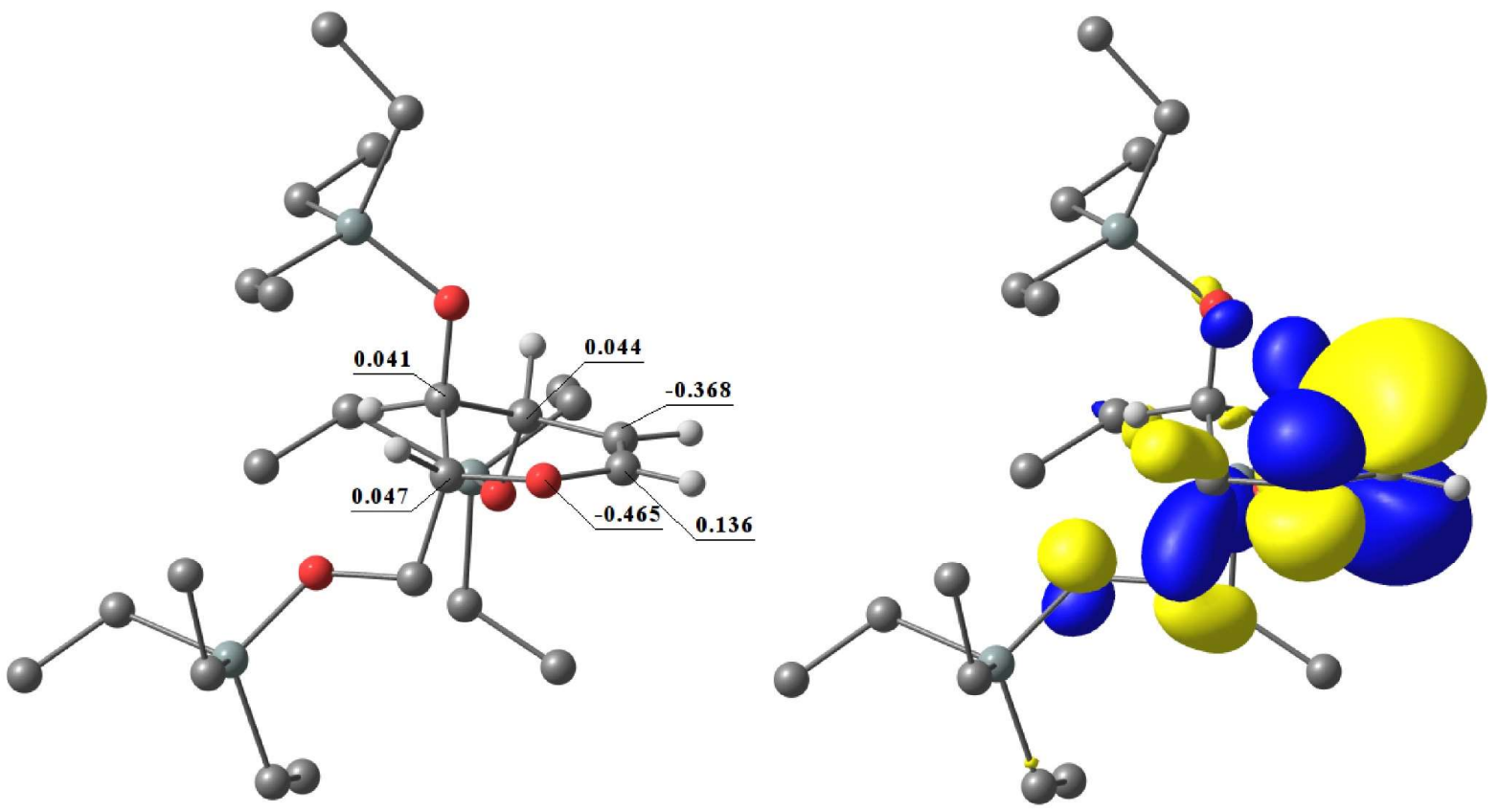

Figure S7. Lowest energy ${ }^{5} \mathrm{H}_{4}$ conformer [PBE0/DEF2-TZVP/SMD(ACN)] of 5 with ring atoms labeled with natural charges. HOMO isosurface shown on the right.
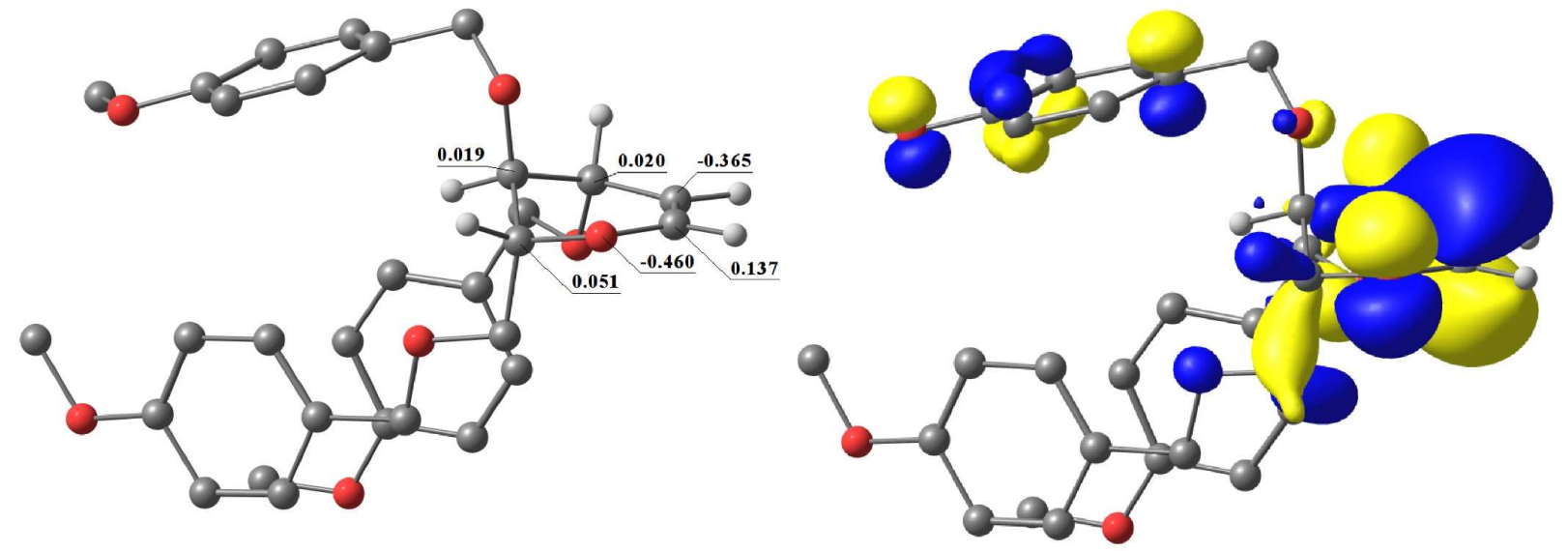

Figure S8. Lowest energy 5H4 conformer [PBE0/DEF2-TZVP/SMD(ACN)] of 6 with ring atoms labeled with natural charges. HOMO-3 isosurface shown on the right. 

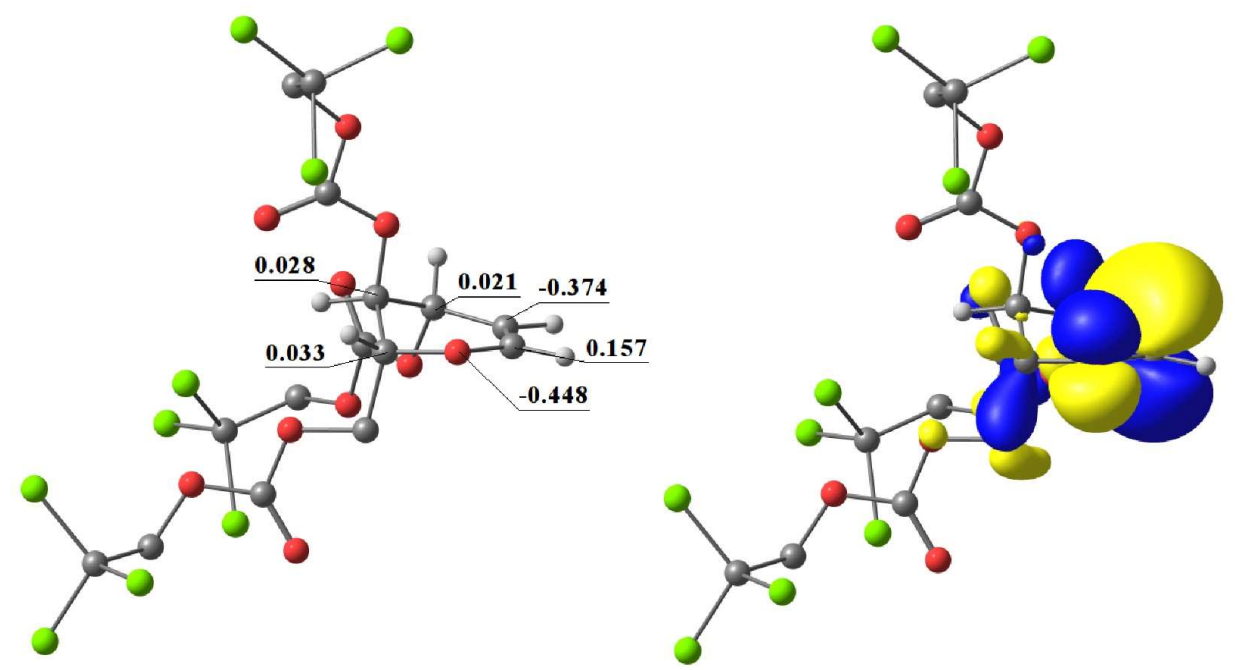

Figure S9. Lowest energy ${ }^{5} \mathrm{H}_{4}$ conformer [PBE0/DEF2-TZVP/SMD(ACN)] of 7 with ring atoms labeled with natural charges. HOMO isosurface shown on the right.
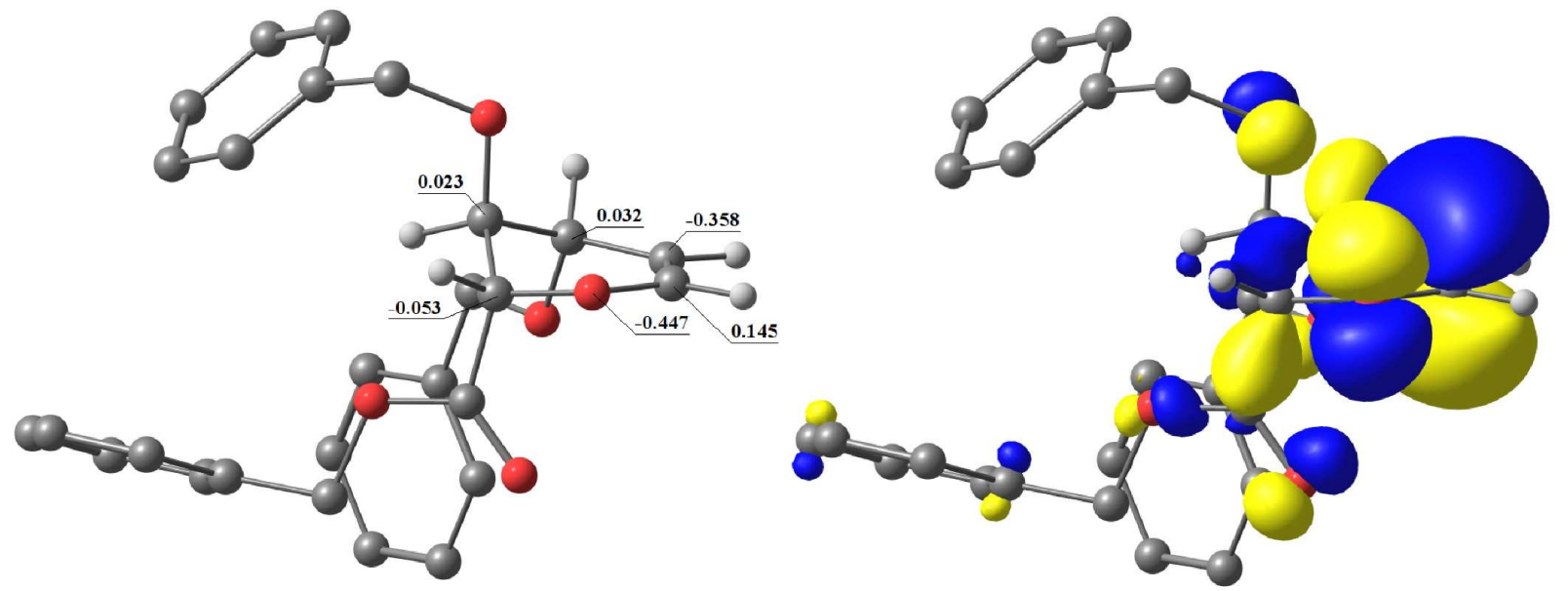

Figure S10. Lowest energy ${ }^{5} \mathrm{H}_{4}$ conformer [PBE0/DEF2-TZVP/SMD(ACN)] of 9 with ring atoms labeled with natural charges. HOMO isosurface shown on the right. 

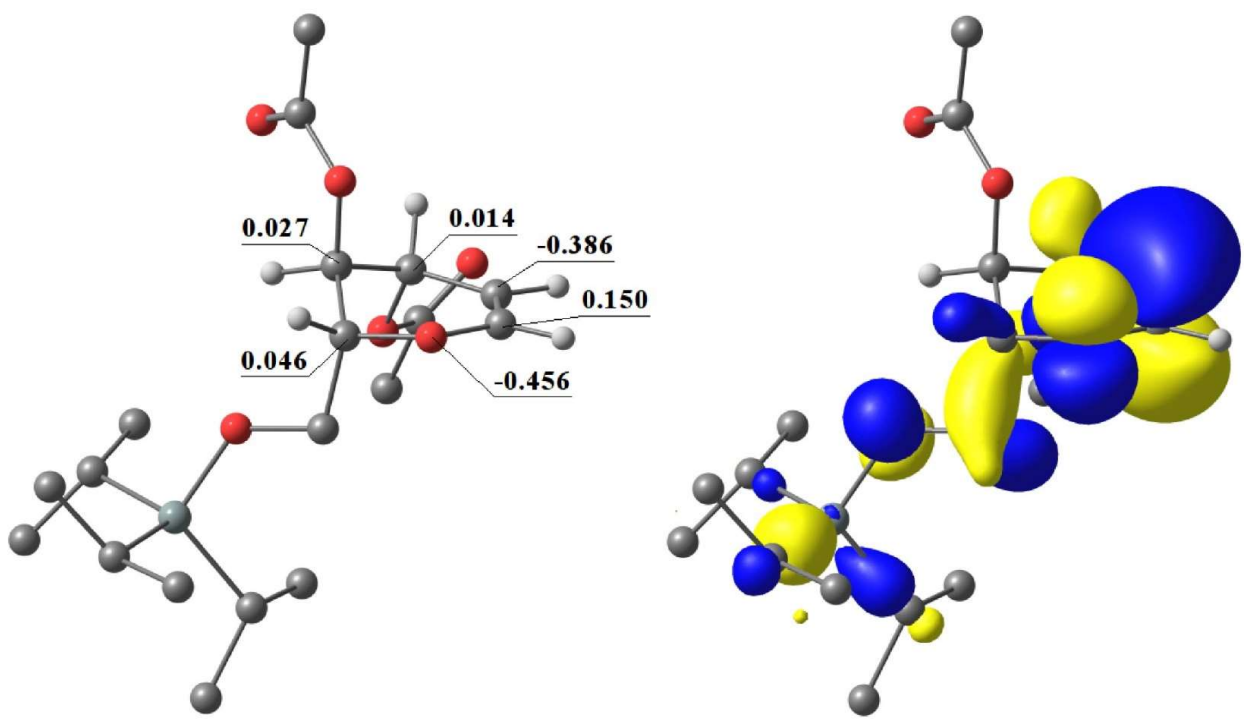

Figure S11. Lowest energy ${ }^{5} \mathrm{H}_{4}$ conformer [PBE0/DEF2-TZVP/SMD(ACN)] of $\mathbf{1 0}$ with ring atoms labeled with natural charges. HOMO isosurface shown on the right.
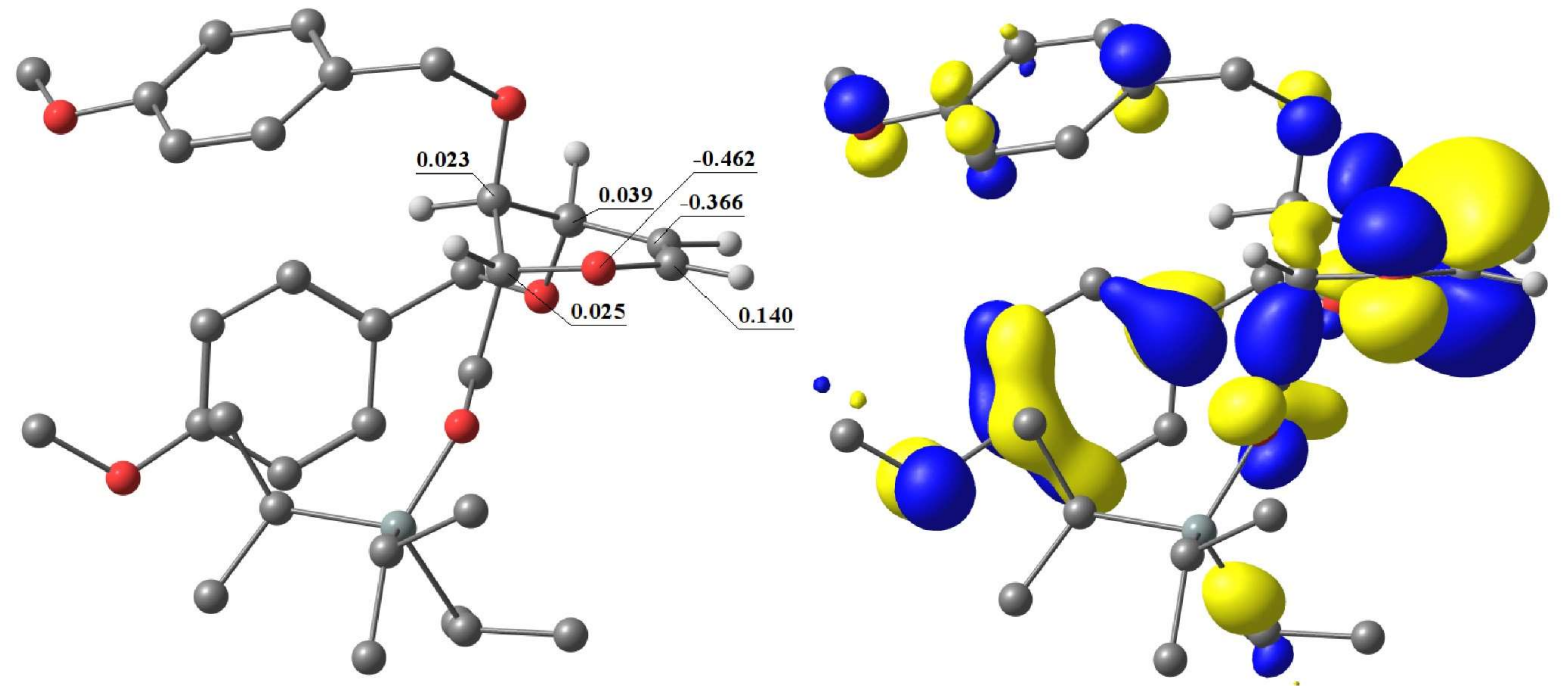

Figure S12. Lowest energy ${ }^{5} \mathrm{H}_{4}$ conformer [PBE0/DEF2-TZVP/SMD(ACN)] of 11 with ring atoms labeled with natural charges. HOMO-2 isosurface shown on the right. 

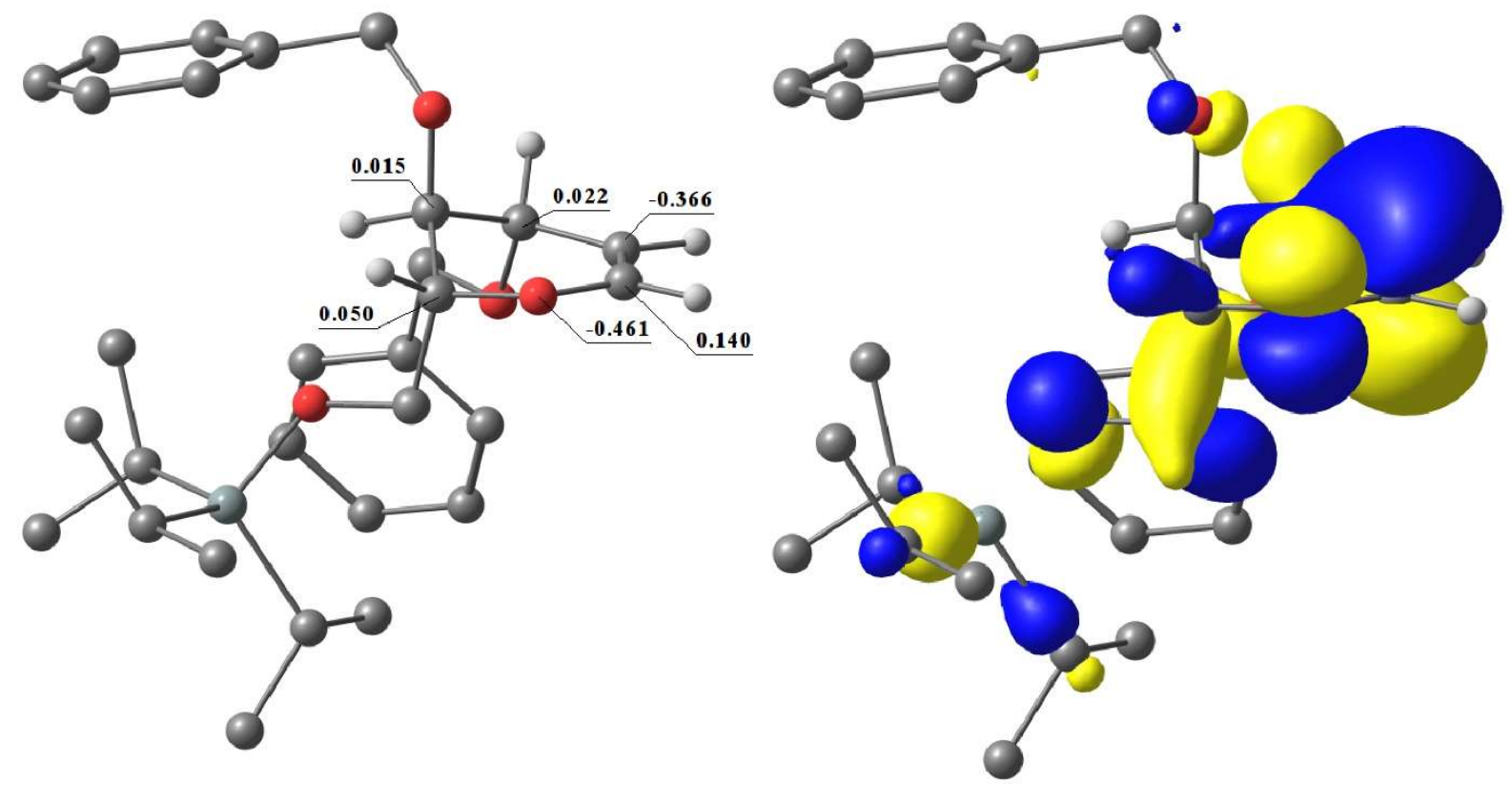

Figure S13. Lowest energy ${ }^{5} \mathrm{H}_{4}$ conformer [PBE0/DEF2-TZVP/SMD(ACN)] of 12 with ring atoms labeled with natural charges. HOMO isosurface shown on the right.
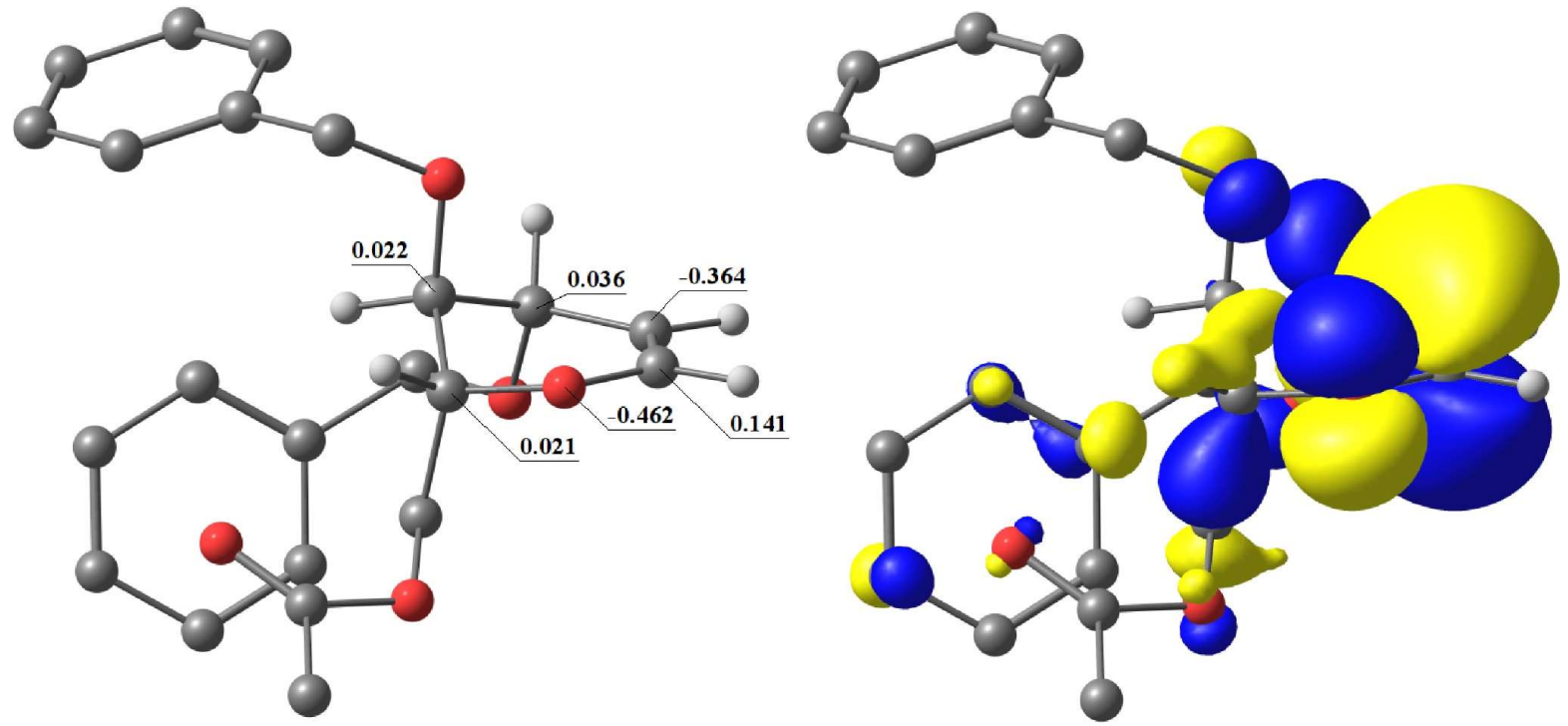

Figure S14. Lowest energy ${ }^{5} \mathrm{H}_{4}$ conformer [PBE0/DEF2-TZVP/SMD(ACN)] of 13 with ring atoms labeled with natural charges. HOMO isosurface shown on the right. 

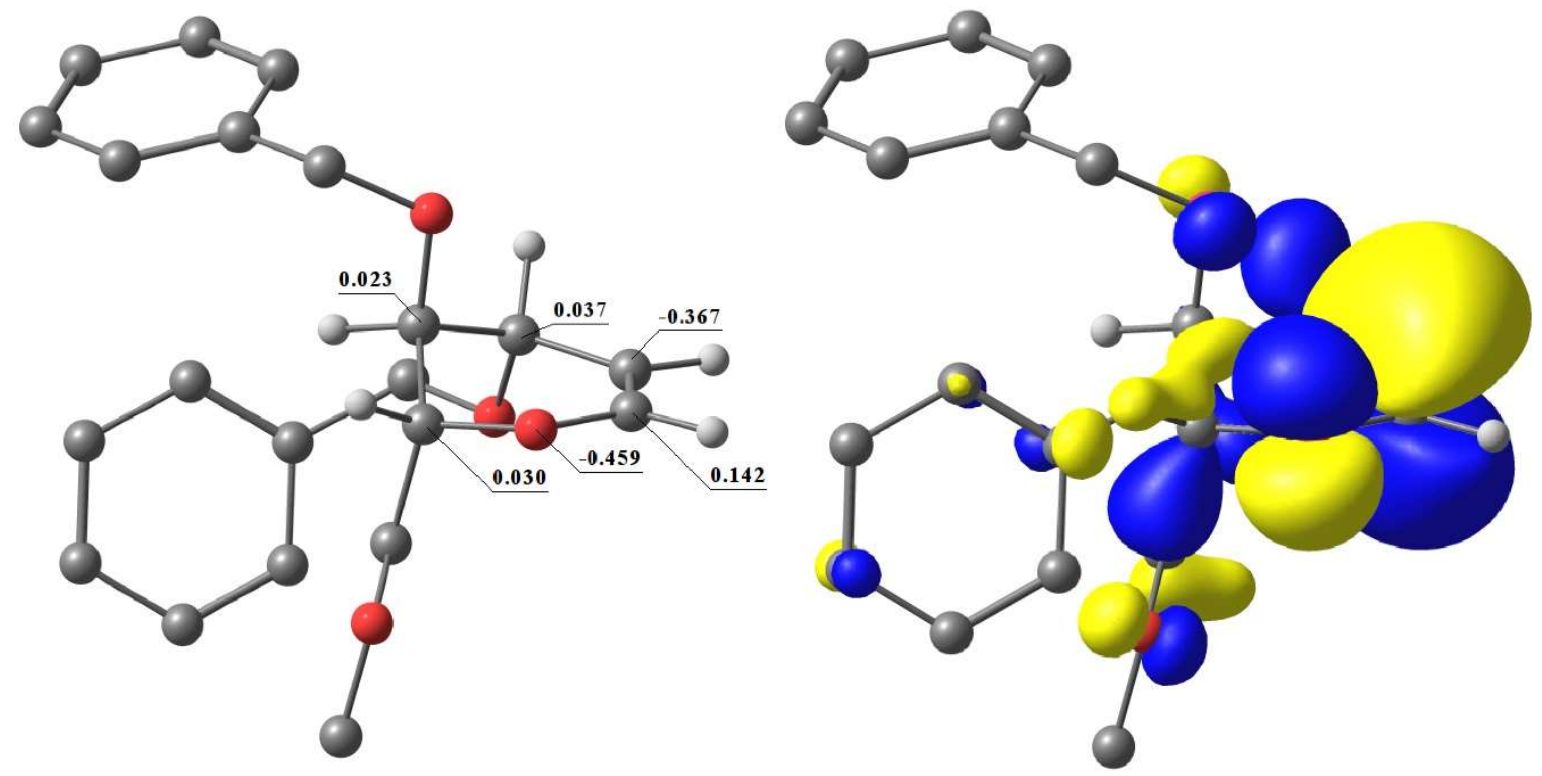

Figure S15. Lowest energy ${ }^{5} \mathrm{H}_{4}$ conformer [PBE0/DEF2-TZVP/SMD(ACN)] of 14 with ring atoms labeled with natural charges. HOMO isosurface shown on the right.
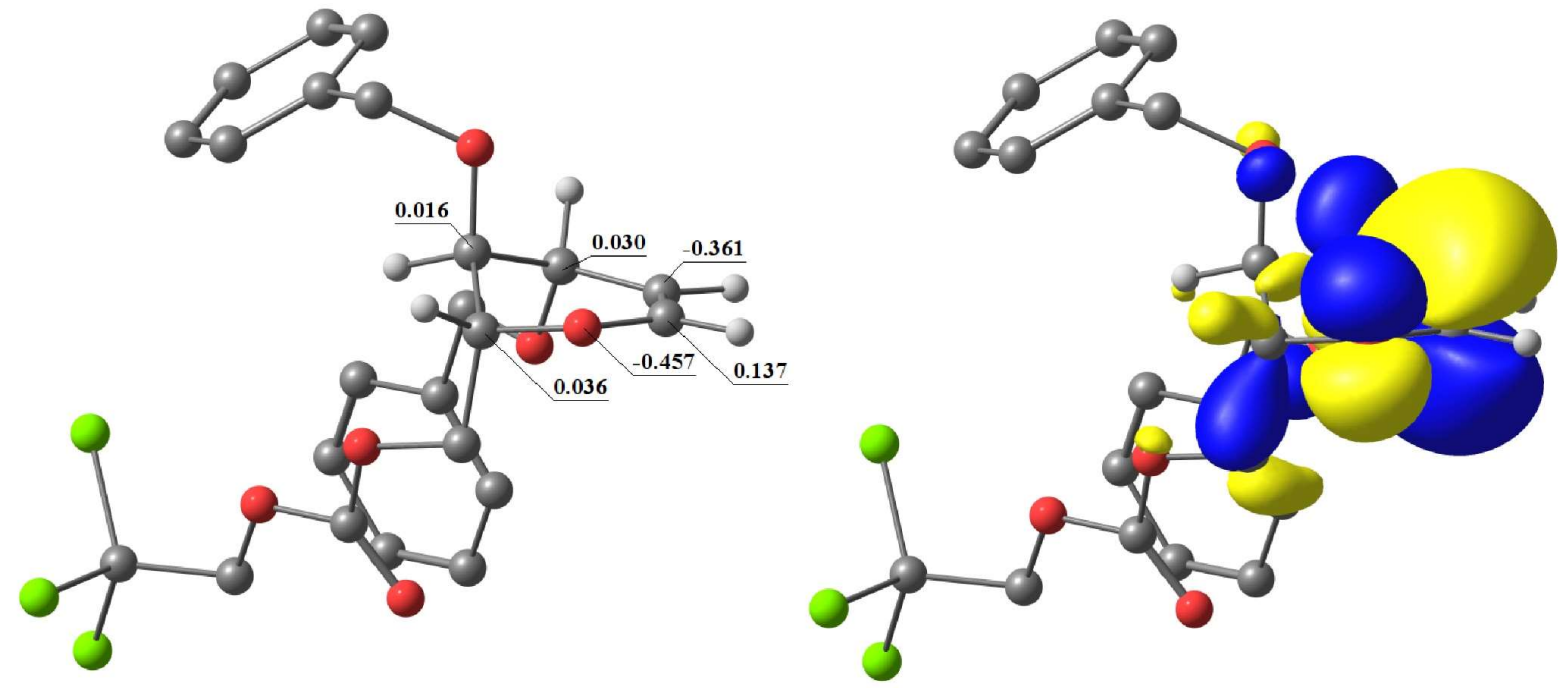

Figure S16. Lowest energy ${ }^{5} \mathrm{H}_{4}$ conformer [PBE0/DEF2-TZVP/SMD(ACN)] of 15 with ring atoms labeled with natural charges. HOMO isosurface shown on the right. 
Figure S17. Comparison of correlation between ${ }^{5} \mathrm{H}_{4}$ and ${ }^{4} \mathrm{H}_{5}$ conformers

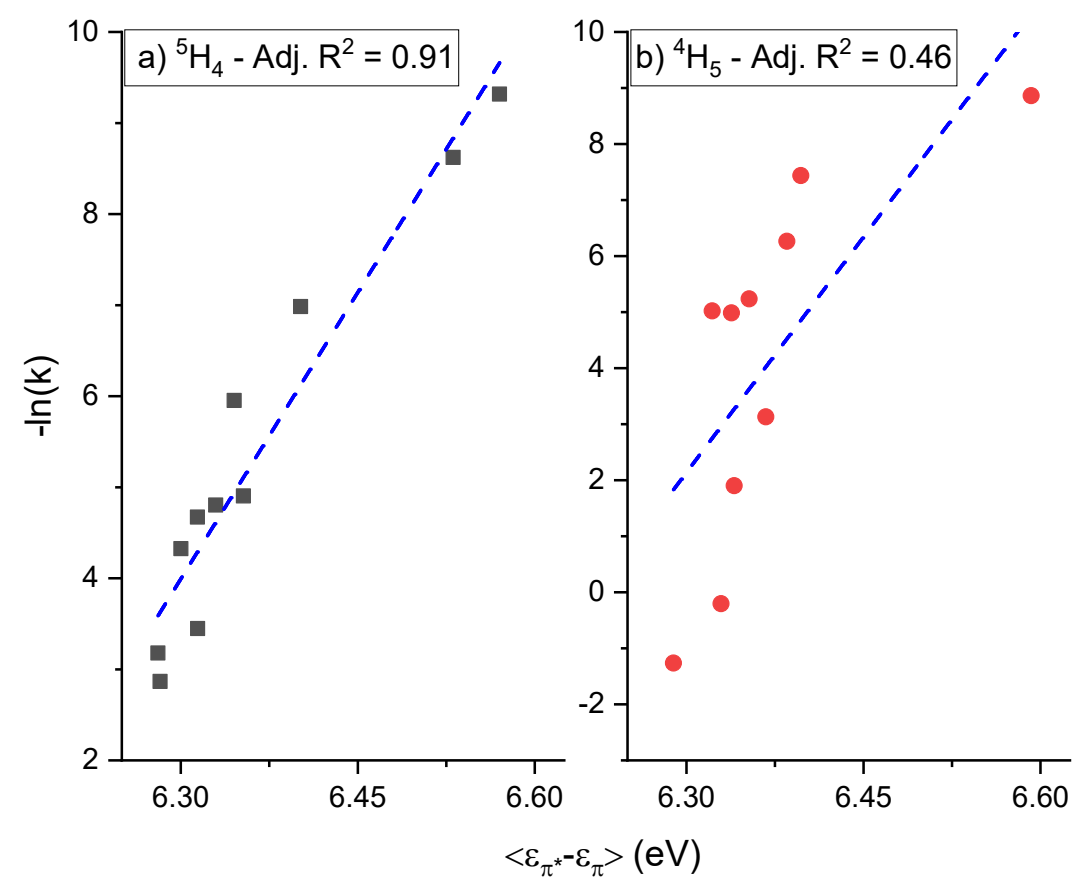

Figure S17 - Comparison of correlation for pseudo-axially-oriented groups versus pseudoequatorially-oriented groups. 
Figure S18. ${ }^{4} \mathrm{H}_{5}$ glycal transition state geometries

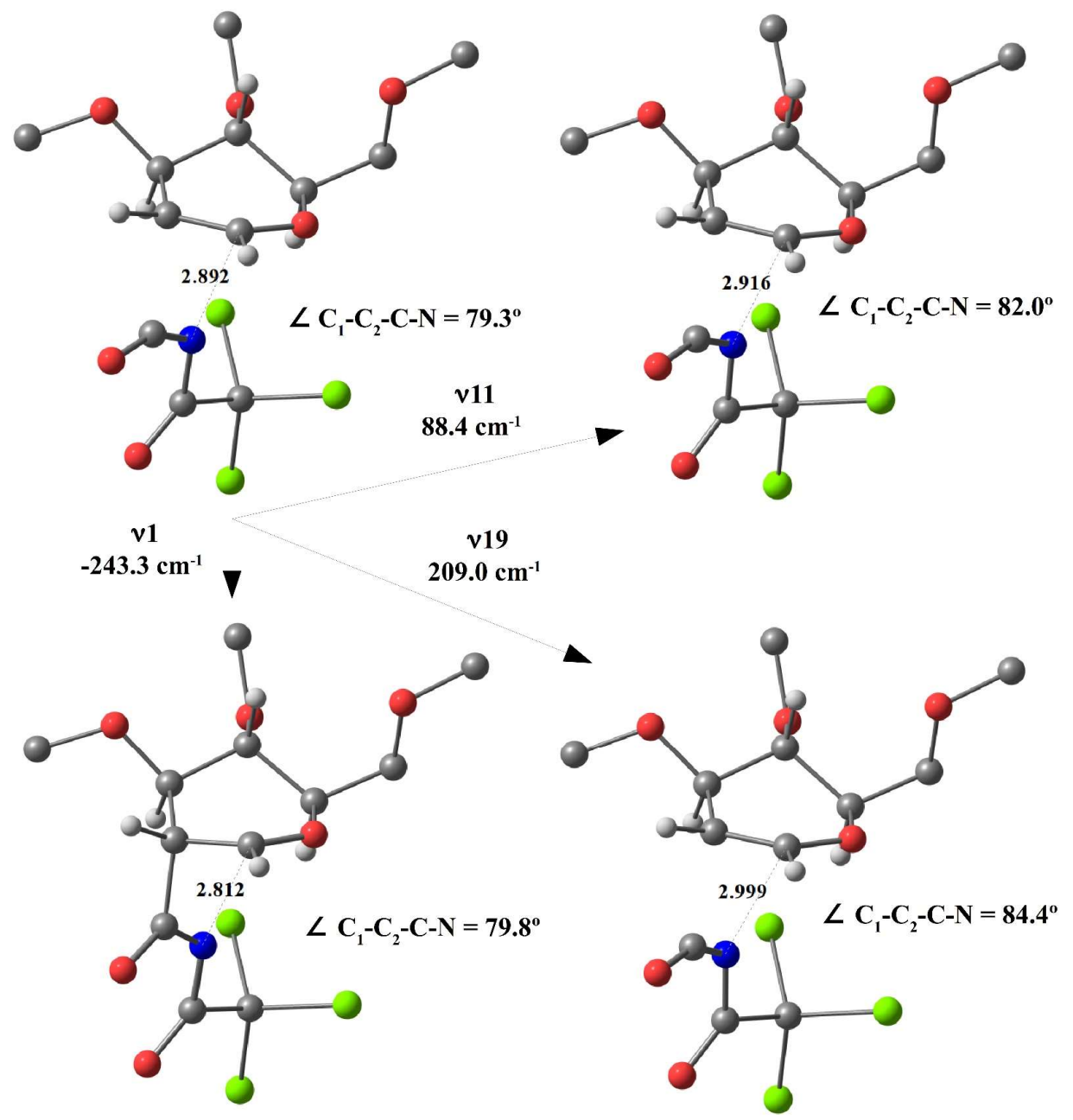

Figure S18 - Displaced geometries, scaled by 0.5, along competing normal modes for the cycoaddition of ${ }^{4} \mathrm{H}_{5}$ with TCAI. 
Figure S19. Comparison of ${ }^{5} \mathrm{H}_{4}$ IRC between gas phase and implicit solvation model

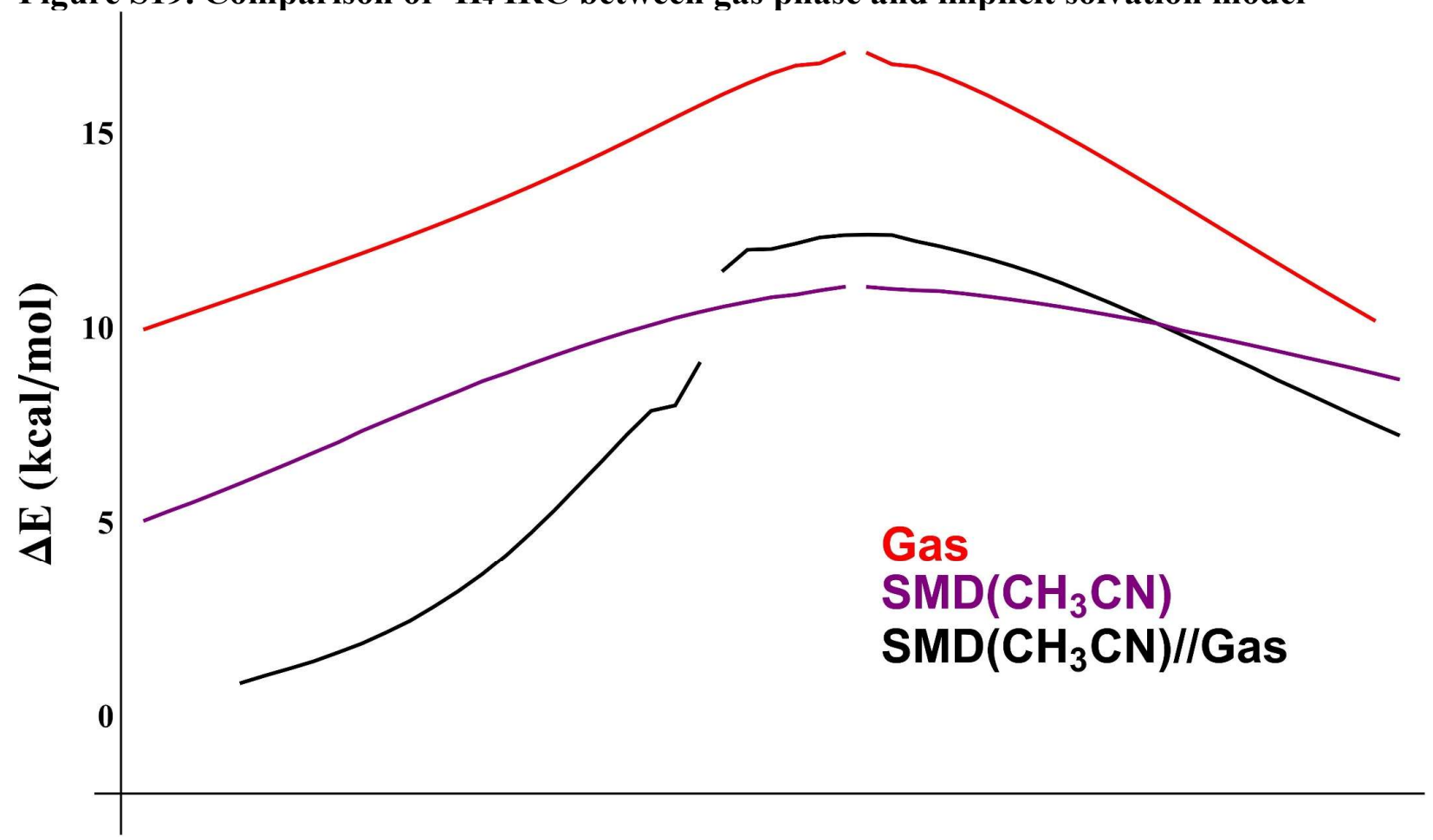

Figure S19 - Portion of the IRC for 3 calculated at the PBE0/DEF2-SVP/D3BJ/RIJCOSX, $\mathrm{PBE}$ /DEF2-SVP/D3BJ/SMD $\left(\mathrm{CH}_{3} \mathrm{CN}\right) / \mathrm{RIJCOSX}$, and PBE0/DEF2-SVP/D3BJ/SMD $\left(\mathrm{CH}_{3} \mathrm{CN}\right)$ /RIJCOSX//PBE0/DEF2-SVP/D3BJ/RIJCOSX levels. 
Figure S20. Comparison of IRC for selected glycal ${ }^{5} \mathrm{H}_{4}$ conformers

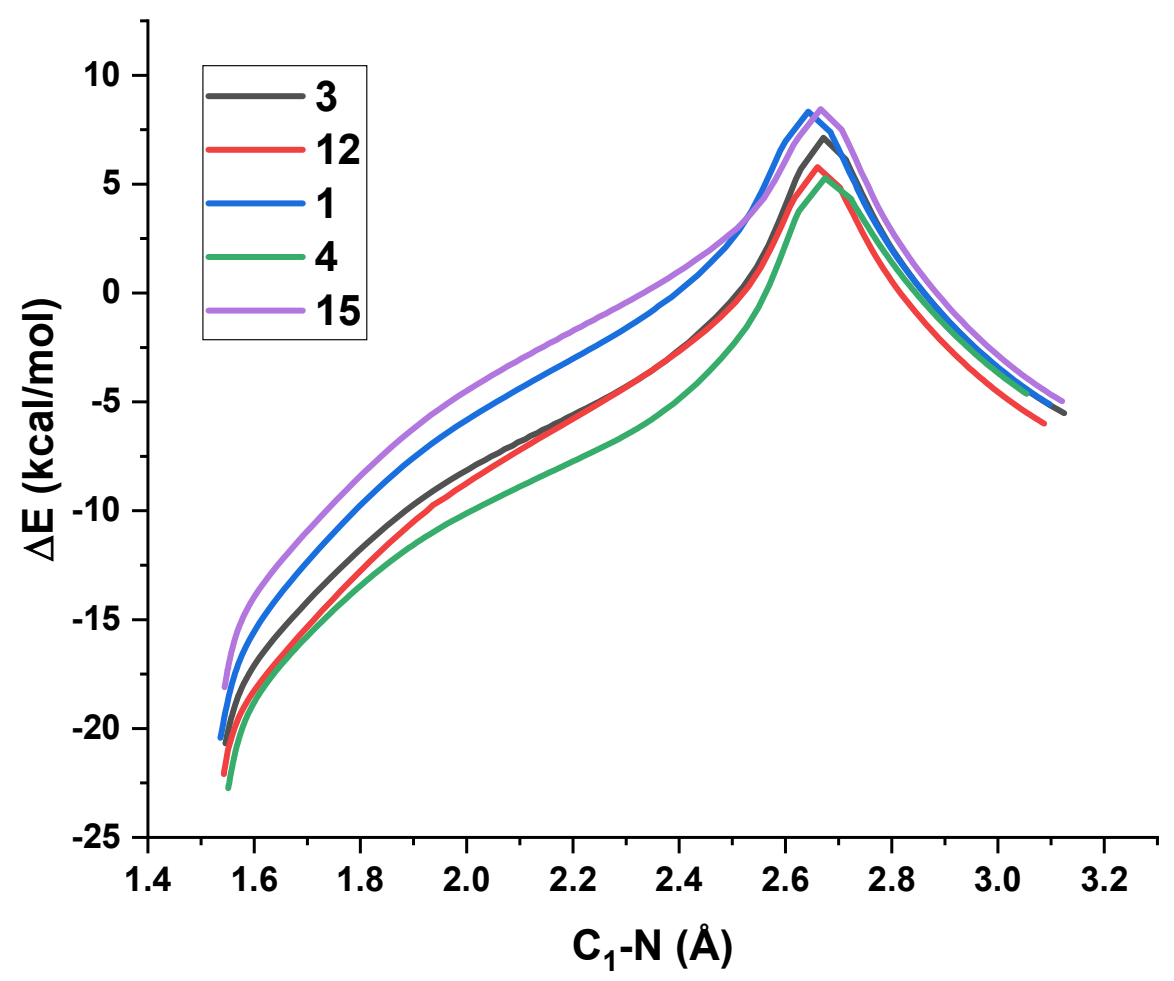

Figure S20 - IRC of the ${ }^{5} \mathrm{H}_{4}$ conformers of $1,3,4,12$, and 15 monitored against the forming $\mathrm{C}_{1}$ $\mathrm{N}$ bond at the PBE0/DEF2-SVP/D3BJ/CPCM $(\mathrm{CH} 3 \mathrm{CN}) / \mathrm{RIJCOSX}$ level. 
Figure S21. Correlation between NBO energy and reaction transition state energy for minimum energy ${ }^{5} \mathrm{H}_{4}$ conformer of selected glycals

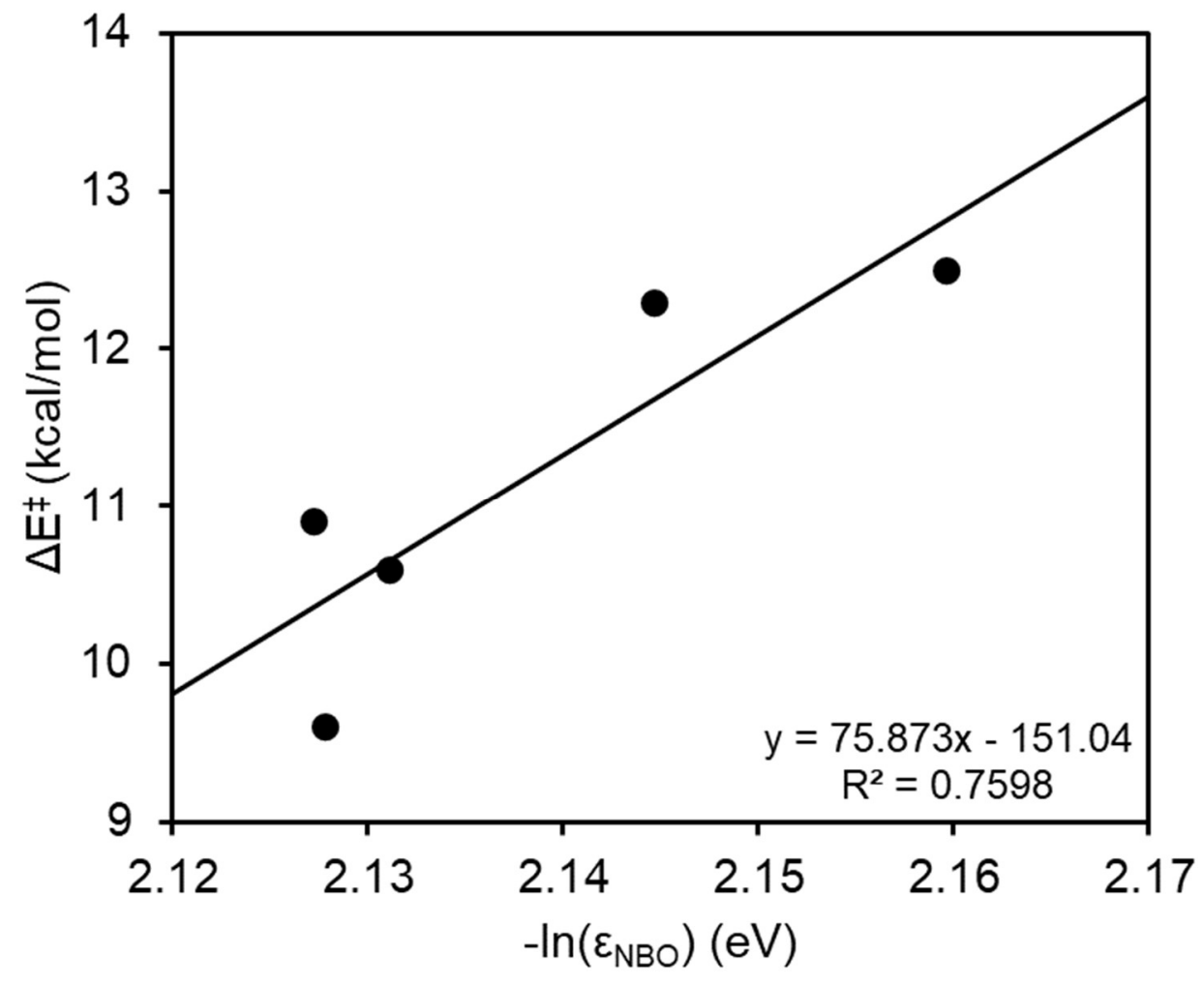


Figure S22. Correlation between $[4+2]$ to $[2+2]$ product ratio and NBO energy gap

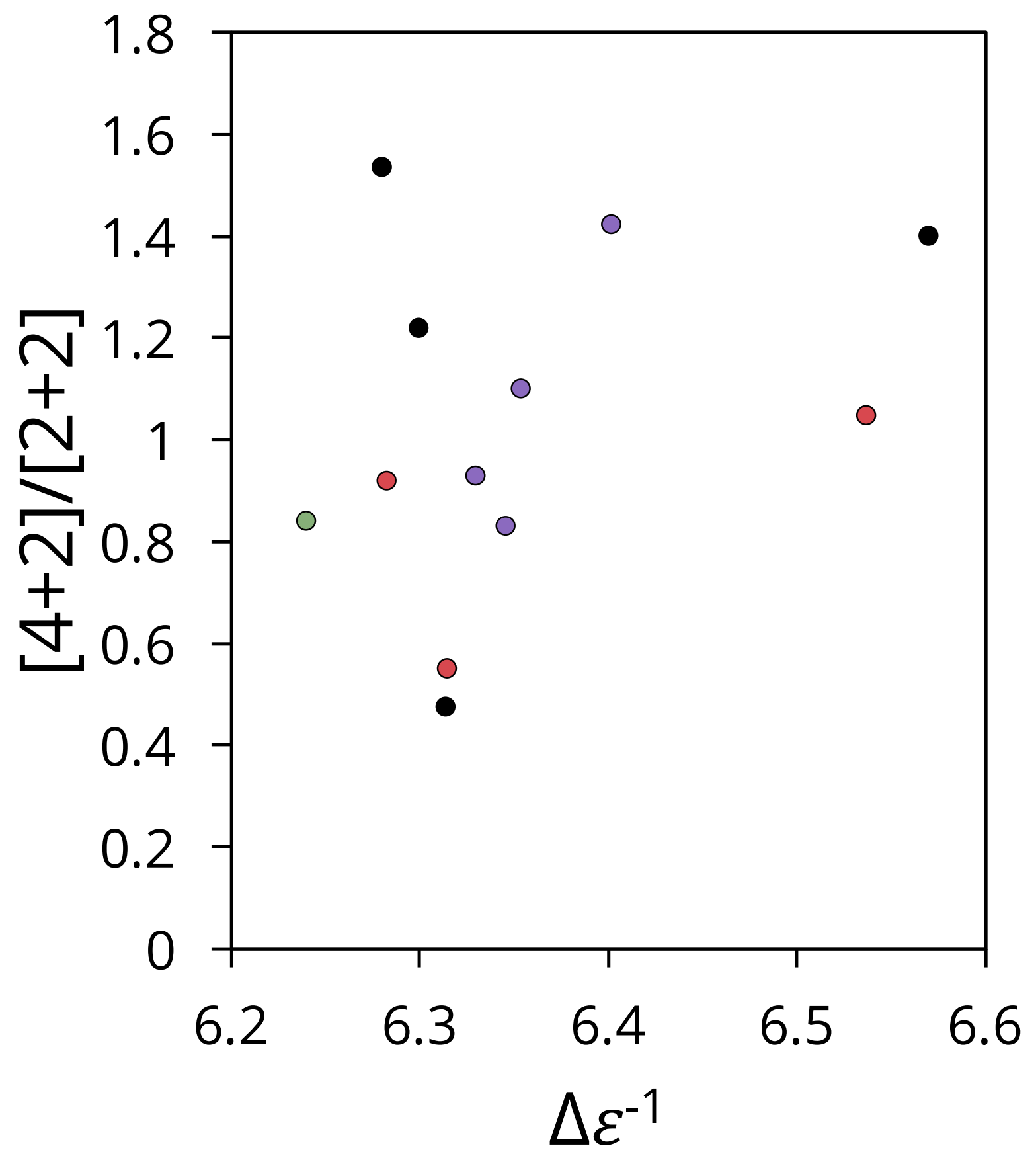

Figure S22. Correlation between the [4+2] to [2+2] ratio (obtained from ${ }^{1} \mathrm{H}-\mathrm{NMR}$ after either $90 \%$ consumption of starting material or $24 \mathrm{~h}$ ) of all 13 glycals and their calculated NBO energy gaps. No relationship is observed between the calculated NBO energy gap and the $[4+2]$ to $[2+2]$ ratio. 
Figure S23. Transition state dynamics of ${ }^{5} \mathrm{H}_{4}$ vs ${ }^{4} \mathrm{H}_{5}$ conformers
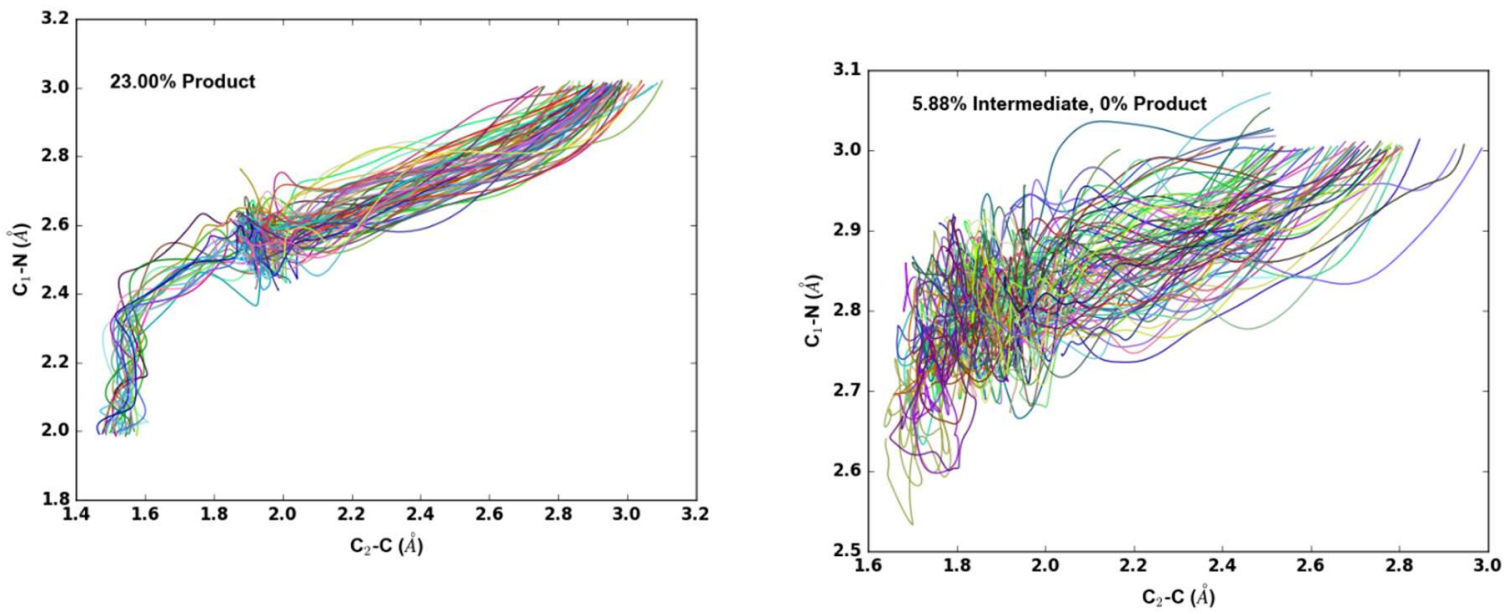

Figure S23. Quasiclassical trajectories for the ${ }^{5} \mathrm{H}_{4}$ (left) and ${ }^{4} \mathrm{H}_{5}$ (right) conformers of 3. 
Figure S24-S28. Comparison of correlation models

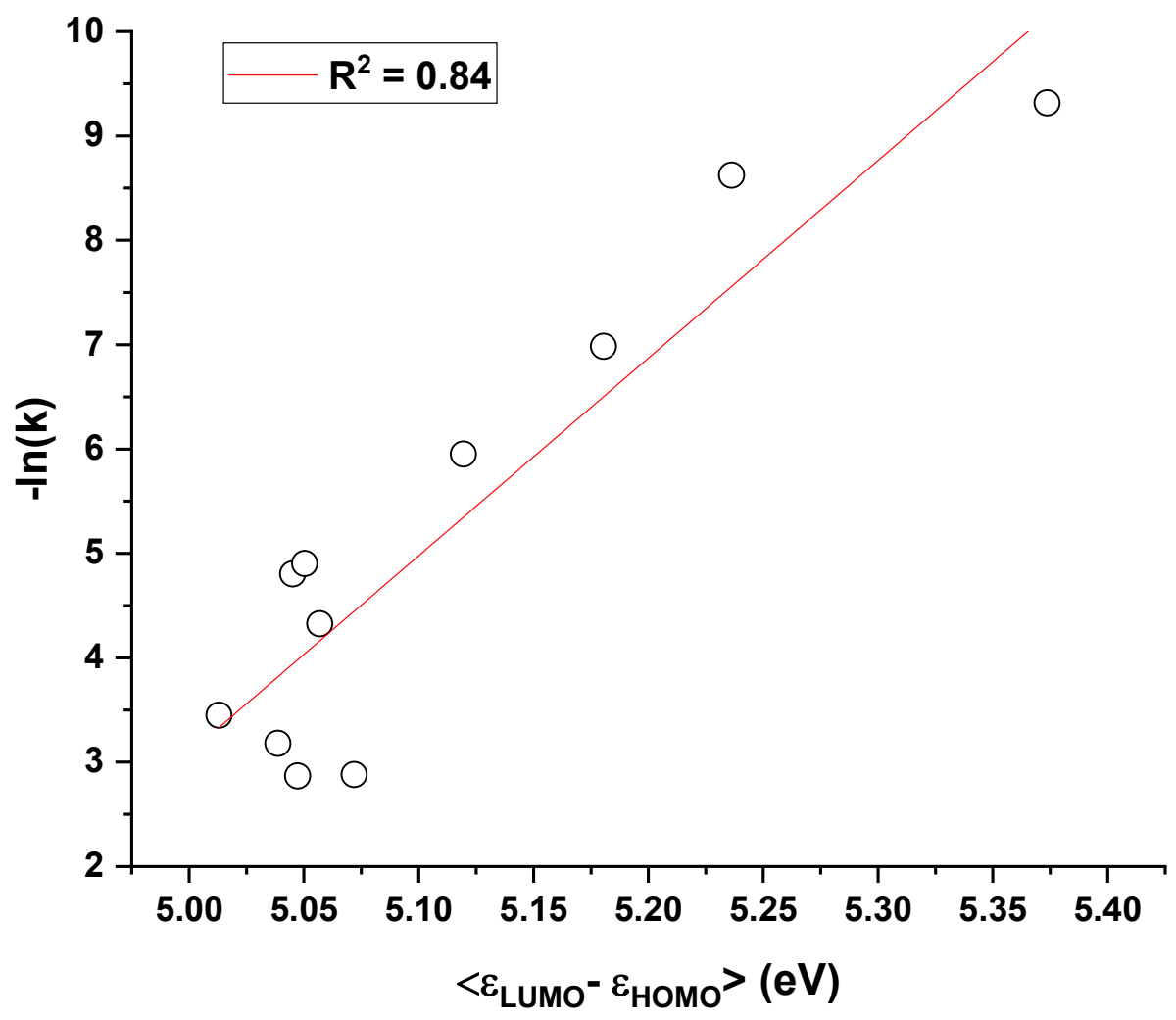

Figure S24. Correlation between experimental $-\ln \left(\mathrm{k}_{\mathrm{A}}\right)$ and the Boltzmann-weighted HOMOLUMO gap for the ${ }^{5} \mathrm{H}_{4}$ conformers of the substrates at the PBE0/DEF2$\mathrm{TZVP} / \mathrm{D} 3 \mathrm{BJ} / \mathrm{CPCM}\left(\mathrm{CH}_{3} \mathrm{CN}\right) / / \mathrm{PBE} 0 / \mathrm{DEF} 2-\mathrm{SVP} / \mathrm{D} 3 \mathrm{BJ} / \mathrm{RIJCOSX}$ level. 


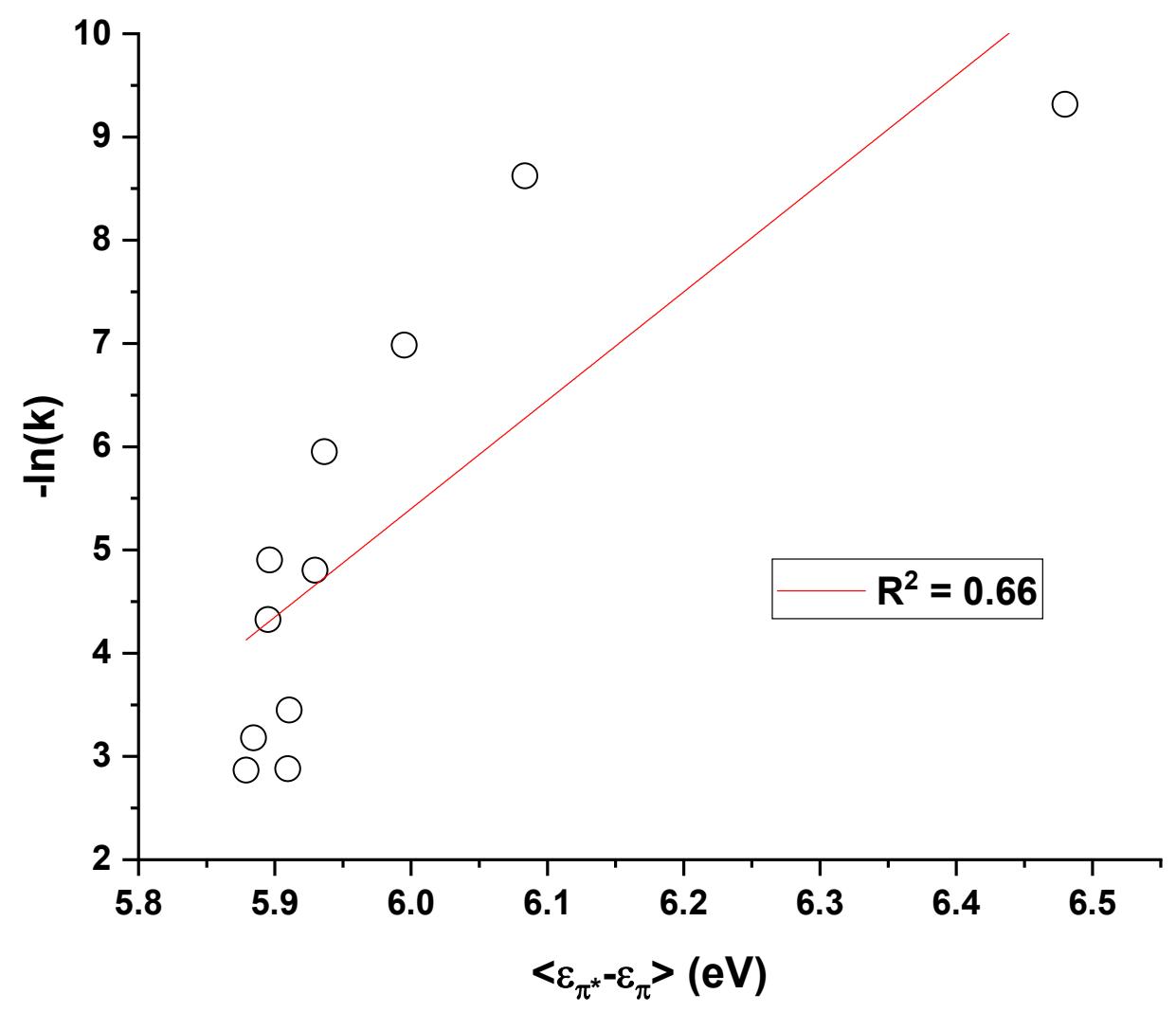

Figure S25. Correlation between experimental $-\ln \left(\mathrm{k}_{\mathrm{A}}\right)$ and the Boltzmann-weighted NBO $\pi^{*}$ TCAI $/ \pi_{\mathrm{C} 1-\mathrm{C} 2}$ gap uncorrected for $\pi \rightarrow \sigma^{*}$ delocalizations for the ${ }^{5} \mathrm{H}_{4}$ conformers of the substrates at the PBE0/DEF2-TZVP/D3BJ/CPCM $\left(\mathrm{CH}_{3} \mathrm{CN}\right) / / \mathrm{PBE} 0 / \mathrm{DEF} 2-\mathrm{SVP} / \mathrm{D} 3 \mathrm{BJ} / \mathrm{RIJCOSX}$ level. 


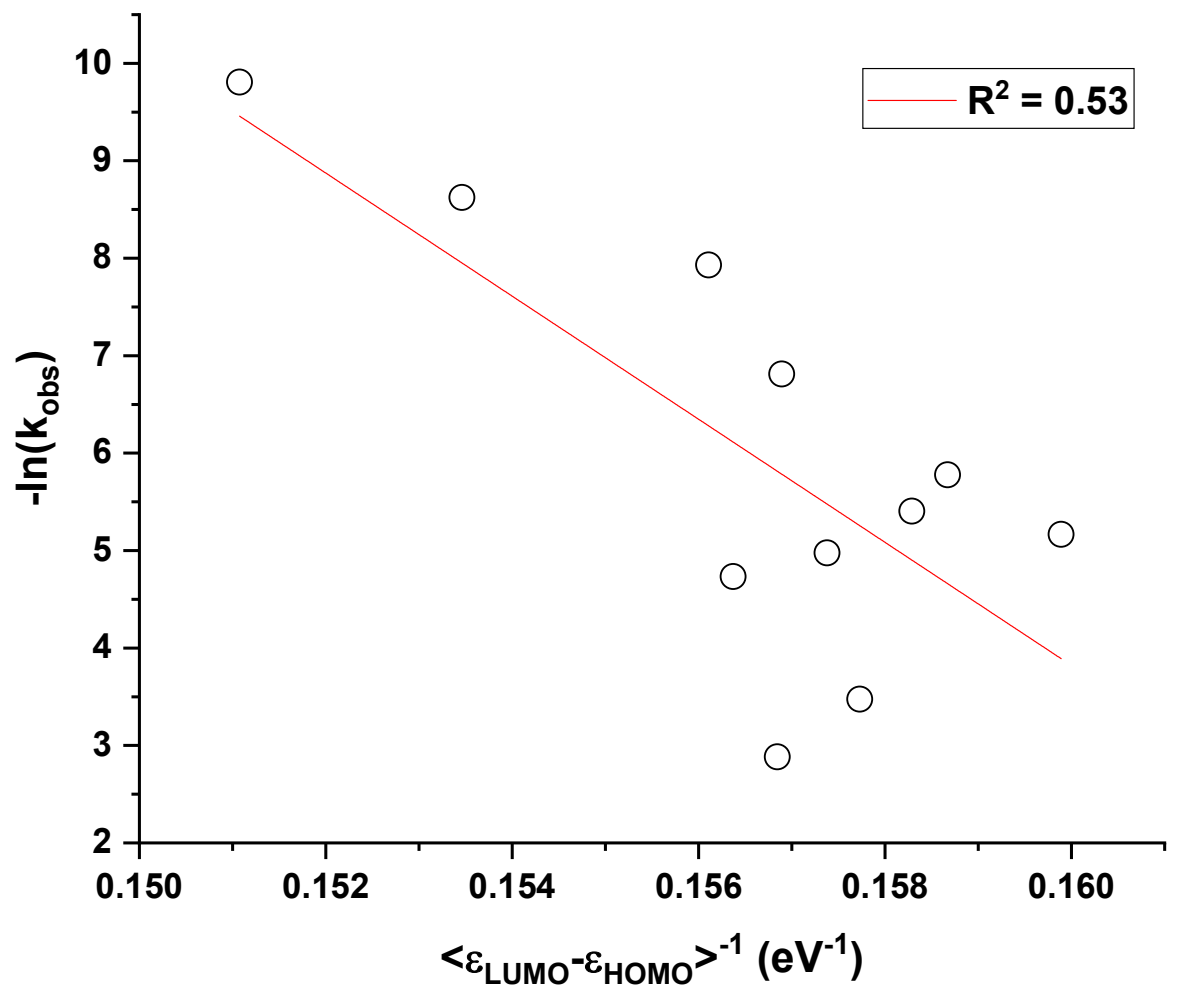

Figure S26. Correlation between experimental $-\ln \left(\mathrm{k}_{\mathrm{obs}}\right)$ and the Boltzmann-weighted HOMOLUMO gap for all conformers of the substrates at the PBE0/DEF2-TZVP/D3BJ/CPCM $\left(\mathrm{CH}_{3} \mathrm{CN}\right)$ //PBE0/DEF2-SVP/D3BJ/RIJCOSX level. 


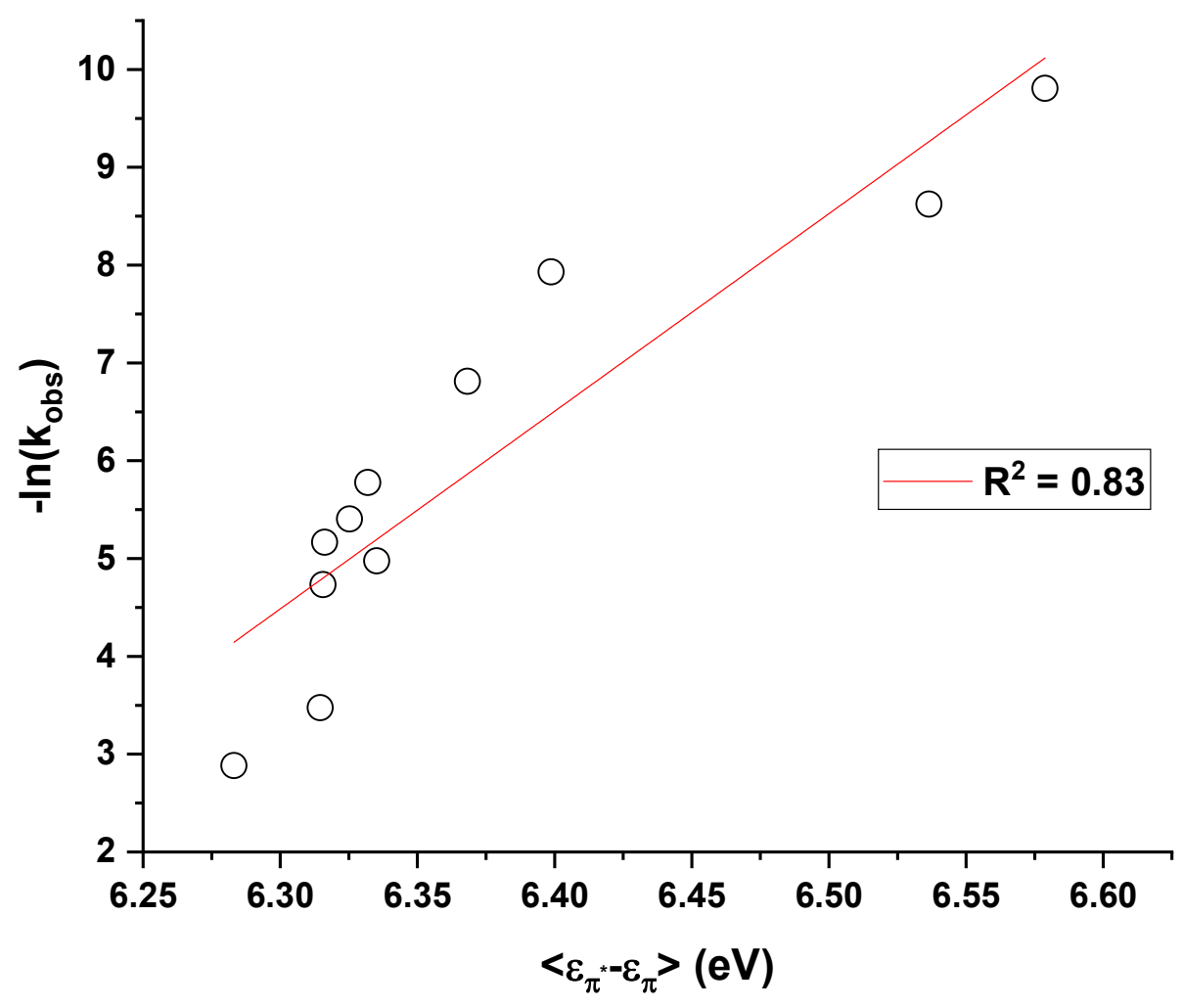

Figure S27. Correlation between experimental $-\ln \left(\mathrm{k}_{\mathrm{obs}}\right)$ and the Boltzmann-weighted NBO $\pi^{*}{ }_{\mathrm{TCAI}} / \pi_{\mathrm{C} 1-\mathrm{C} 2}$ gap corrected for $\pi \rightarrow \sigma^{*}$ delocalizations for all conformers of the substrates at the $\mathrm{PBE} 0 / \mathrm{DEF} 2-\mathrm{TZVP} / \mathrm{D} 3 \mathrm{BJ} / \mathrm{CPCM}\left(\mathrm{CH}_{3} \mathrm{CN}\right) / / \mathrm{PBE} 0 / \mathrm{DEF} 2-\mathrm{SVP} / \mathrm{D} 3 \mathrm{BJ} / \mathrm{RIJCOSX}$ level. 


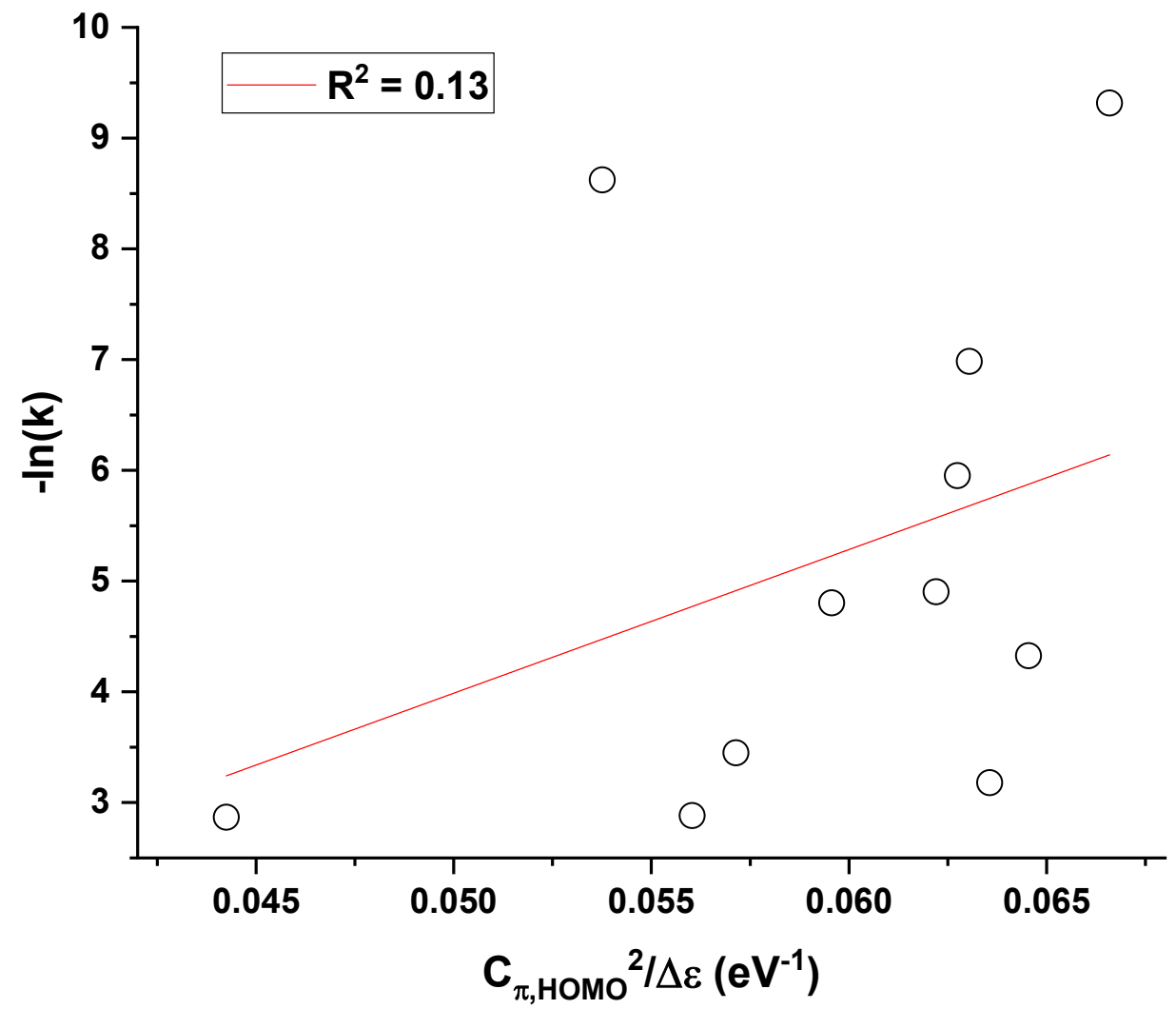

Figure S28. Correlation between $-\ln \left(\mathrm{k}_{\mathrm{A}}\right)$ and the Boltzmann-weighted ${ }^{5} \mathrm{H}_{4} \mathrm{HOMO}$ energies at the PBE0/DEF2-TZVP/D3BJ/CPCM $\left(\mathrm{CH}_{3} \mathrm{CN}\right) / / \mathrm{PBE} 0 / \mathrm{DEF} 2-\mathrm{SVP} / \mathrm{D} 3 \mathrm{BJ} / \mathrm{RIJCOSX}$ level corrected for the $\pi$-bond expansion coefficients as determined from $\mathrm{CMO}$ analyses. 


\section{Supplementary Methods}

\section{Adjustment of experimental reaction rate for ${ }^{5} \mathrm{H}_{4}$ conformer population}

We assume that each substrate is populated by 2 conformers: pseudo-axial (A) and pseudoequatorial (E). Therefore, it follows that the total concentration of glycal $(\mathrm{G})$ in solution is:

$$
[G]=[A]+[E]
$$

Through computational transition state modeling and reaction dynamics, we observe that conformers $\mathrm{A}$ and conformers $\mathrm{E}$ undergo the [2+2] cycloaddition reaction at differing rates, $k_{\mathrm{A}}$ and $k_{\mathrm{E}}$ and that $k_{\mathrm{A}} \gg k_{\mathrm{E}}$. We additionally assume that these rate differences stem from overall glycal reactivity in conformation A or E; $k_{\mathrm{A}}>>k_{\mathrm{E}}$ for the [4+2] cycloaddition reaction as well. In support of this assumption, the ratio between $\mathrm{A}$ and $\mathrm{E}$ (determined by conformational sampling) does not correlate with the [4+2] to [2+2] ratio found to be constant over the period of reaction monitoring. Therefore, the observed rate of consumption for $\mathrm{G}$ is equal to:

$$
\frac{d[G]}{d t}=k_{o b s} *[G]
$$

Additionally:

$$
\frac{d[A]}{d t}=k_{A}[A] ; \frac{d[E]}{d t}=k_{E}[E]
$$

Therefore:

$$
\frac{d[G]}{d t}=\frac{d[A]}{d t}+\frac{d[E]}{d t}=k_{A}[A]+k_{E}[E]
$$

Since $k_{\mathrm{A}}>k_{\mathrm{E}}$,

$$
\frac{d[G]}{d t}=k_{A}[A]
$$

$\rho$ is defined as the fraction of $\mathrm{A}$ in the total mixture, determined by conformational sampling.

$$
k_{o b s} *[G]=k_{A}[A]
$$




$$
k_{o b s}=k_{A} \rho
$$

To account for the ${ }^{5} \mathrm{H}_{4}$ conformer population in the reaction mixture of the NMR experiment, the observed reaction rate is now adjusted by conformational sampling:

$$
k_{A}=k_{a d j}=\frac{k_{o b s}}{\rho}
$$

\section{Computational Methods}

\section{General Computational Methods}

We began our computational investigation by performing conformational scans on each of the reactive substrates $(\mathbf{1 - 7}, \mathbf{9 - 1 5})$ with Tinker $8 .{ }^{1}$ Atom types were assigned using the sdf2tinkerxyz ${ }^{2}$ utility, and the conformational scans were run with the MMFF94 force field. ${ }^{3-7}$ Following these scans, $2.5 \mathrm{kcal} / \mathrm{mol}$ windows were retained for 1-4, 6-7, 9-10, and 12-15. Due to the high conformation density of $\mathbf{5}$ and $\mathbf{1 1}$ these windows were further constrained to 1.2 and $2.0 \mathrm{kcal} / \mathrm{mol}$ respectively.

The conformational windows defined above were further optimized at the PBE0 ${ }^{8-10} / \mathrm{DEF} 2$ $\mathrm{SVP}^{11} / \mathrm{D} 3 \mathrm{BJ}{ }^{12,13} / \mathrm{RIJCOSX}^{14-16}$ level using the Orca electronic structure package, version $4^{17,18}$, with the DEF $2 / \mathrm{J}^{19}$ auxiliary basis set for evaluation of the Coulomb integrals. The compound DFT integration keyword GRID7 was used along with the GRIDX7 keyword for the numerical integrations evaluated in the RIJCOSX approximation. Each structure was confirmed as a local minimum based on the lack of imaginary harmonic frequencies after Hessian calculations. 
The resulting structures were then subjected to single-point evaluations and natural bond orbital analyses at the PBE0/DEF2-TZVP/D3BJ/CPCM $\left(\mathrm{CH}_{3} \mathrm{CN}\right)^{20-23}$ level using the quantum chemistry package Firefly ${ }^{24}$ with NBO $5.9^{25}$. We have used version 8 of Firefly, which is partially based on the GAMESS-US source code ${ }^{26}$. The resulting electronic energies and free energies are listed in Tables SCY1-SCY14. The minimum energy ${ }^{5} \mathrm{H}_{4}$ structure is bolded in each of these tables. The free energies listed in these tables include thermodynamic parameters calculated at the PBE0/DEF2-SVP/D3BJ/RIJCOSX level. Figure S19 shows that the model adequately accounts for solvent effects, at least in so far as implicit solvation is suitable. It can be seen that the implicit solvation//gas method correctly brings down the activation barrier for the cycloaddition of $\mathbf{3}$ relative to gas-phase results.

The minimum-energy ${ }^{5} \mathrm{H}_{4}$ and ${ }^{4} \mathrm{H}_{5}$ conformers for each substrate were re-optimized at the PBE0/DEF2-TZVP/D3BJ/RIJCOSX and PBE0/DEF2-TZVP/D3BJ/SMD $\left(\mathrm{CH}_{3} \mathrm{CN}\right) / \mathrm{RIJCOSX}$ levels with Orca, and the resulting orbital energies are those listed in the main text in Table 2.

\section{Transition State Modeling}

Transition states for the cycloaddition of the lowest-energy ${ }^{5} \mathrm{H}_{4}$ and ${ }^{4} \mathrm{H}_{5}$ conformers of $\mathbf{1 , 3 , 4 , 1 2}$, and 15 were located at the PBE0/DEF2-SVP/D3BJ/CPCM $\left(\mathrm{CH}_{3} \mathrm{CN}\right)$ level with the Gaussian charge scheme. The same level of theory was used to generate the intrinsic reaction coordinates connecting each transitions state to products and reactants. The energetic summaries are shown in Table SCX1. Intrinsic reaction coordinates for the ${ }^{5} \mathrm{H}_{4}$ conformers of $\mathbf{1}, \mathbf{3}, \mathbf{4}, \mathbf{1 2}$, and $\mathbf{1 5}$ are shown in Figure S20. 
Inspection of Table SCX1 and Figures S20 leads to several further questions as well as some conclusions: 1) The activation barriers for the ${ }^{5} \mathrm{H}_{4}$ conformers as determined from $\Delta G_{\text {act }}=$ $G_{T S}-G_{T C A I}-G_{X}$, where $G_{X}$ is the free energy of the applicable substrate, appear to correlate with $\epsilon_{\pi_{C_{1}-C_{2}}}$. Here $\epsilon_{\pi_{C_{1}-C_{2}}}$ is the energy of the localized glycal $\pi$-bond as determined by the NBO analyses. This immediately suggests that an FMO-like analysis of these reactions is applicable, thus supporting our conclusions in the main text; 2) As was discussed in the main text, Table SCX1 suggests that the cycloadditions should be much more rapid for the ${ }^{4} \mathrm{H}_{5}$ conformers as compared with the ${ }^{5} \mathrm{H}_{4}$ conformers. However, this did not agree with simple steric considerations that predicted the stereoselectivity observed experimentally to be diminished in the ${ }^{4} \mathrm{H}_{5}$ conformers. In addition, the correlation between experimental rate constants and valence energies was significantly reduced for the ${ }^{4} \mathrm{H}_{5}$ conformers as can be seen in Figure $\mathrm{S} 17$ where the $\mathrm{R}^{2}$ is reduced from 0.91 to 0.46 .

We thus posited that dynamics effects were present that rendered canonical transition state theory inoperable for the comparison between these systems. A preliminary direct dynamics study has thus been initiated that has so far supported our claim that the dynamics of the ${ }^{4} \mathrm{H}_{5}$ cycloaddition render it much slower than the corresponding ${ }^{5} \mathrm{H}_{4}$ cycloaddition, thus allowing us to correlate our observed reaction rates with the ${ }^{5} \mathrm{H}_{4}$ conformers only. A summary of the results is shown in Figure S22.

The direct dynamics were performed in the gas phase with a program that is based heavily upon $\operatorname{ProgDyn}^{27,28}$ from Singleton et al. DFT energies and gradients were calculated with Orca at the 
PBE0/DEF2-SV(P)/D3BJ/RIJCOSX level. This program distributes weighted energies into the normal modes, which are then used to displace the atoms in random phase and amplitude. The dynamics were then determined according to the Verlet algorithm using $1 \mathrm{fs}$ timesteps. The results reported here are preliminary and will be expanded upon in a future publication.

\section{Additional Correlation Models Explored}

In addition to the model correlation model reported in the main text and described above, we have also assessed the correlation of the observed reaction rates with a variety of other electronic factors. These models are shown and described in Figures S23-S27. It should be noted here that we also observed very nice correlation between the observed rate constant (not corrected for ${ }^{4} \mathrm{H}_{5} /{ }^{5} \mathrm{H}_{4}$ concentration) and the NBO gap uncorrected for delocalization into neighboring $\sigma^{*}$ orbitals, but we chose not to use this model because it seemed less theoretically robust than the model described in the manuscript.

\section{References}

(1) Rackers, J. A.; Wang, Z.; Lu, C.; Laury, M. L.; Lagardère, L.; Schnieders, M. J.;

Piquemal, J.-P.; Ren, P.; Ponder, J. W. Tinker 8: Software Tools for Molecular Design. J.

Chem. Theory Comput. 2018, 14 (10), 5273-5289.

https://doi.org/10.1021/acs.jctc.8b00529.

(2) Tosco, P.; Balle, T.; Shiri, F. SDF2XYZ2SDF: How to Exploit TINKER Power in Cheminformatics Projects. J. Mol. Model. 2011, 17 (11), 3021. https://doi.org/10.1007/s00894-011-1111-7.

(3) Halgren, T. A. Merck Molecular Force Field. I. Basis, Form, Scope, Parameterization, and 
Performance of MMFF94. J. Comput. Chem. 1996, 17 (5-6), 490-519. https://doi.org/10.1002/(SICI)1096-987X(199604)17:5/6<490::AID-JCC1>3.0.CO;2-P.

(4) Halgren, T. A. Merck Molecular Force Field. II. MMFF94 van Der Waals and Electrostatic Parameters for Intermolecular Interactions. J. Comput. Chem. 1996, 17 (5-6), 520-552. https://doi.org/10.1002/(SICI)1096-987X(199604)17:5/6<520::AID$\mathrm{JCC} 2>3.0 . \mathrm{CO} ; 2-\mathrm{W}$.

(5) Halgren, T. A. Merck Molecular Force Field. III. Molecular Geometries and Vibrational Frequencies for MMFF94. J. Comput. Chem. 1996, 17 (5-6), 553-586. https://doi.org/10.1002/(SICI)1096-987X(199604)17:5/6<553::AID-JCC3>3.0.CO;2-T.

(6) Halgren, T. A.; Nachbar, R. B. Merck Molecular Force Field. IV. Conformational Energies and Geometries for MMFF94. J. Comput. Chem. 1996, 17 (5-6), 587-615. https://doi.org/10.1002/(SICI)1096-987X(199604)17:5/6<587::AID-JCC4>3.0.CO;2-Q.

(7) Halgren, T. A. Merck Molecular Force Field. V. Extension of MMFF94 Using Experimental Data, Additional Computational Data, and Empirical Rules. J. Comput. Chem. 1996, 17 (5-6), 616-641. https://doi.org/10.1002/(SICI)1096987X(199604)17:5/6<616::AID-JCC5>3.0.CO;2-X.

(8) Perdew, J. P.; Ernzerhof, M.; Burke, K. Rationale for Mixing Exact Exchange with Density Functional Approximations. J. Chem. Phys. 1996, 105 (22), 9982-9985. https://doi.org/10.1063/1.472933.

(9) Adamo, C.; Barone, V. Toward Reliable Density Functional Methods without Adjustable Parameters: The PBE0 Model. J. Chem. Phys. 1999, 110 (13), 6158-6170. https://doi.org/10.1063/1.478522.

(10) Becke, A. D. A New Mixing of Hartree-Fock and Local Density-functional Theories. J. 
Chem. Phys. 1993, 98 (2), 1372-1377. https://doi.org/10.1063/1.464304.

(11) Weigend, F.; Ahlrichs, R. Balanced Basis Sets of Split Valence, Triple Zeta Valence and Quadruple Zeta Valence Quality for H to Rn: Design and Assessment of Accuracy. Phys. Chem. Chem. Phys. 2005, 7 (18), 3297-3305. https://doi.org/10.1039/B508541A.

(12) Grimme, S.; Ehrlich, S.; Goerigk, L. Effect of the Damping Function in Dispersion Corrected Density Functional Theory. J. Comput. Chem. 2011, 32 (7), 1456-1465. https://doi.org/10.1002/jcc.21759.

(13) Grimme, S.; Antony, J.; Ehrlich, S.; Krieg, H. A Consistent and Accurate Ab Initio Parametrization of Density Functional Dispersion Correction (DFT-D) for the 94 Elements H-Pu. J. Chem. Phys. 2010, 132 (15), 154104. https://doi.org/10.1063/1.3382344.

(14) Ganyushin, D.; Gilka, N.; Taylor, P. R.; Marian, C. M.; Neese, F. The Resolution of the Identity Approximation for Calculations of Spin-Spin Contribution to Zero-Field Splitting Parameters. J. Chem. Phys. 2010, 132 (14), 144111. https://doi.org/10.1063/1.3367718.

(15) Kossmann, S.; Neese, F. Comparison of Two Efficient Approximate Hartee-Fock Approaches. Chem. Phys. Lett. 2009, 481 (4), 240-243. https://doi.org/https://doi.org/10.1016/j.cplett.2009.09.073.

(16) Neese, F.; Wennmohs, F.; Hansen, A.; Becker, U. Efficient, Approximate and Parallel Hartree-Fock and Hybrid DFT Calculations. A 'Chain-of-Spheres' Algorithm for the Hartree-Fock Exchange. Chem. Phys. 2009, 356 (1), 98-109. https://doi.org/https://doi.org/10.1016/j.chemphys.2008.10.036.

(17) Neese, F. The ORCA Program System. Wiley Interdiscip. Rev. Comput. Mol. Sci. 2012, 2 (1), 73-78. https://doi.org/10.1002/wcms.81. 
(18) Neese, F. Software Update: The ORCA Program System, Version 4.0. Wiley Interdiscip. Rev. Comput. Mol. Sci. 2018, 8 (1), e1327. https://doi.org/10.1002/wcms.1327.

(19) Weigend, F. Accurate Coulomb-Fitting Basis Sets for H to Rn. Phys. Chem. Chem. Phys. 2006, 8 (9), 1057-1065. https://doi.org/10.1039/b515623h.

(20) Klamt, A.; Schüürmann, G. COSMO: A New Approach to Dielectric Screening in Solvents with Explicit Expressions for the Screening Energy and Its Gradient. J. Chem. Soc. Perkin Trans. 2 1993, No. 5, 799-805. https://doi.org/10.1039/P29930000799.

(21) Andzelm, J.; Kölmel, C.; Klamt, A. Incorporation of Solvent Effects into Density Functional Calculations of Molecular Energies and Geometries. J. Chem. Phys. 1995, 103 (21), 9312-9320. https://doi.org/10.1063/1.469990.

(22) Barone, V.; Cossi, M. Quantum Calculation of Molecular Energies and Energy Gradients in Solution by a Conductor Solvent Model. J. Phys. Chem. A 1998, 102 (11), 1995-2001. https://doi.org/10.1021/jp9716997.

(23) Cossi, M.; Rega, N.; Scalmani, G.; Barone, V. Energies, Structures, and Electronic Properties of Molecules in Solution with the C-PCM Solvation Model. J. Comput. Chem. 2003, 24 (6), 669-681. https://doi.org/10.1002/jcc.10189.

(24) Granovsky, A. A. Firefly Version 8.

(25) Glendening, E. D.; Badenhoop, A. E.; Reed, A. E.; Carpenter, J. E.; Bohmann, J. A.; Morales, C. M.; Weinhold, F. NBO 5.9. 2012.

(26) Schmidt, M. W.; Baldridge, K. K.; Boatz, J. A.; Elbert, S. T.; Gordon, M. S.; Jensen, J. H.; Koseki, S.; Matsunaga, N.; Nguyen, K. A.; Su, S.; et al. General Atomic and Molecular Electronic Structure System. J. Comput. Chem. 1993, 14 (11), 1347-1363. https://doi.org/10.1002/jcc.540141112. 
(27) Ussing, B. R.; Hang, C.; Singleton, D. A. Dynamic Effects on the Periselectivity, Rate, Isotope Effects, and Mechanism of Cycloadditions of Ketenes with Cyclopentadiene. $J$. Am. Chem. Soc. 2006, 128 (23), 7594-7607. https://doi.org/10.1021/ja0606024.

(28) Kurouchi, H.; Singleton, D. A. Labelling and Determination of the Energy in Reactive Intermediates in Solution Enabled by Energy-Dependent Reaction Selectivity. Nat. Chem. 2018, 10 (2), 237-241. https://doi.org/10.1038/NCHEM.2907. 


\section{Spectral Characterization of 4-18}

3,4,6-tri-O-ethyl-D-glucal (4) - ${ }^{1} \mathrm{H}-\mathrm{NMR}$

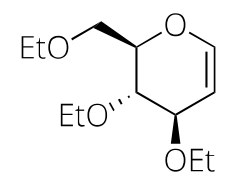

$\mathrm{CDCl}_{3}, 500 \mathrm{MHz}$

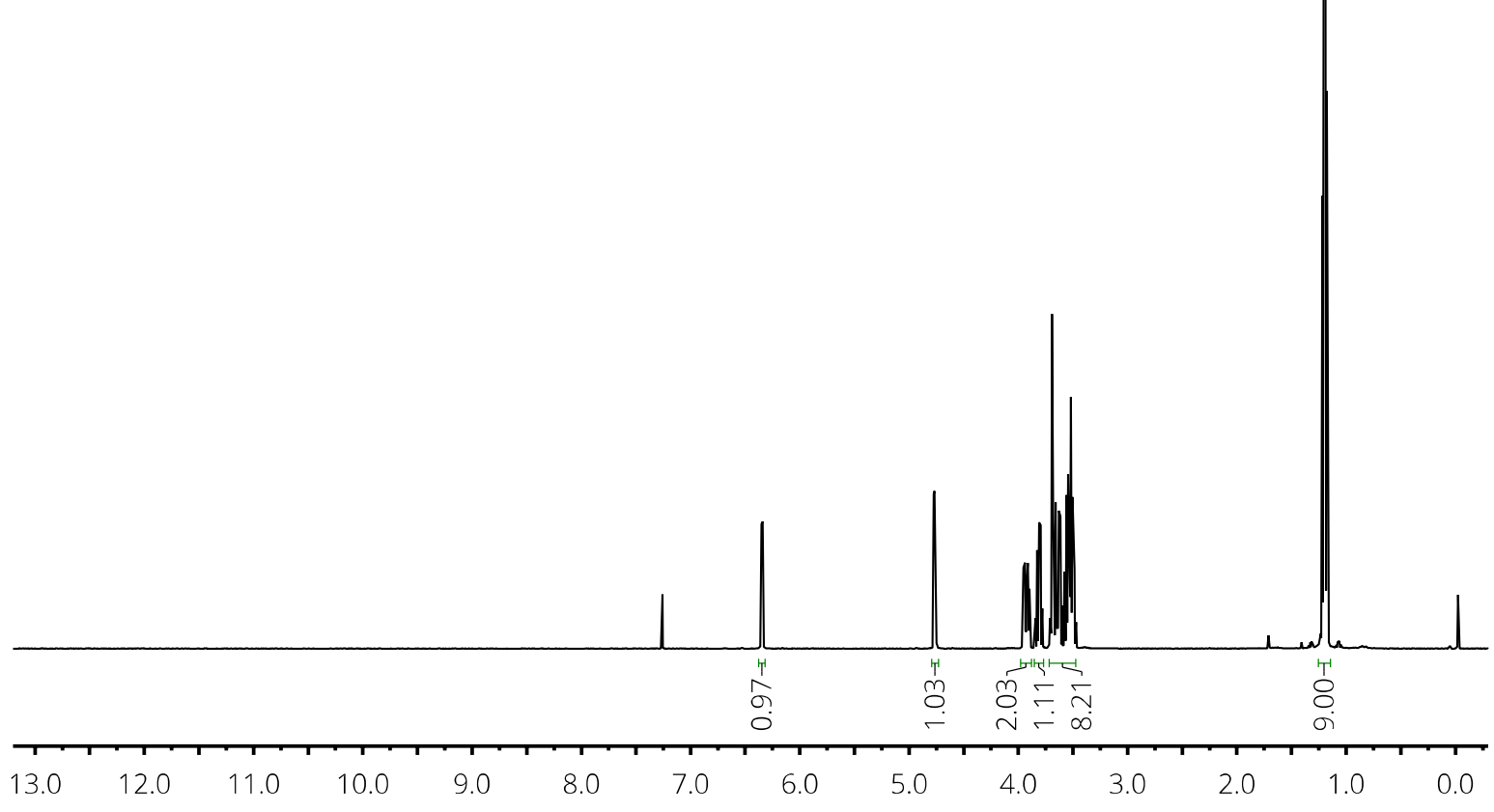


3,4,6-tri-O-ethyl-D-glucal (4) $-{ }^{13} \mathrm{C}\left\{{ }^{1} \mathrm{H}\right\}$-NMR
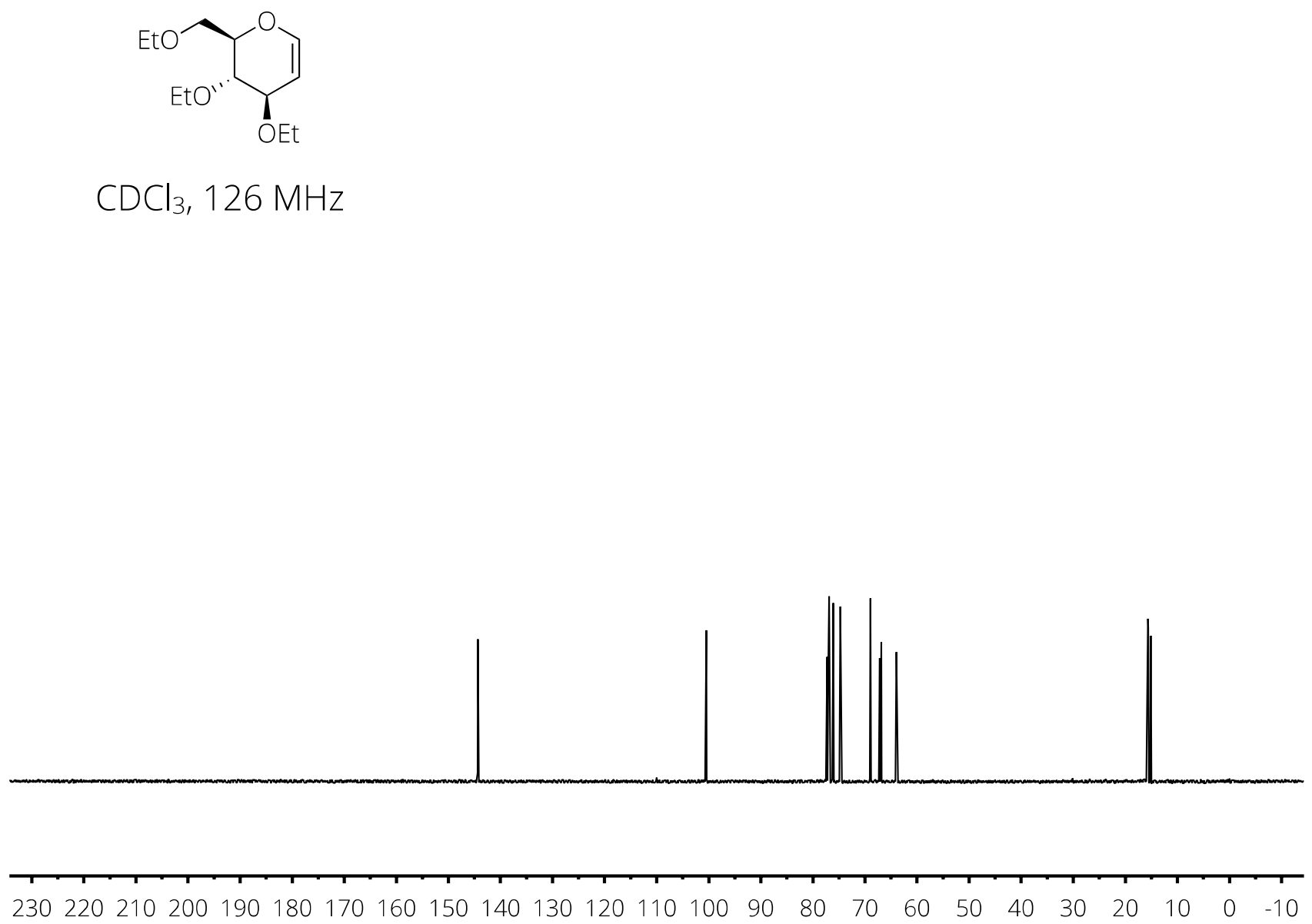

S

38 


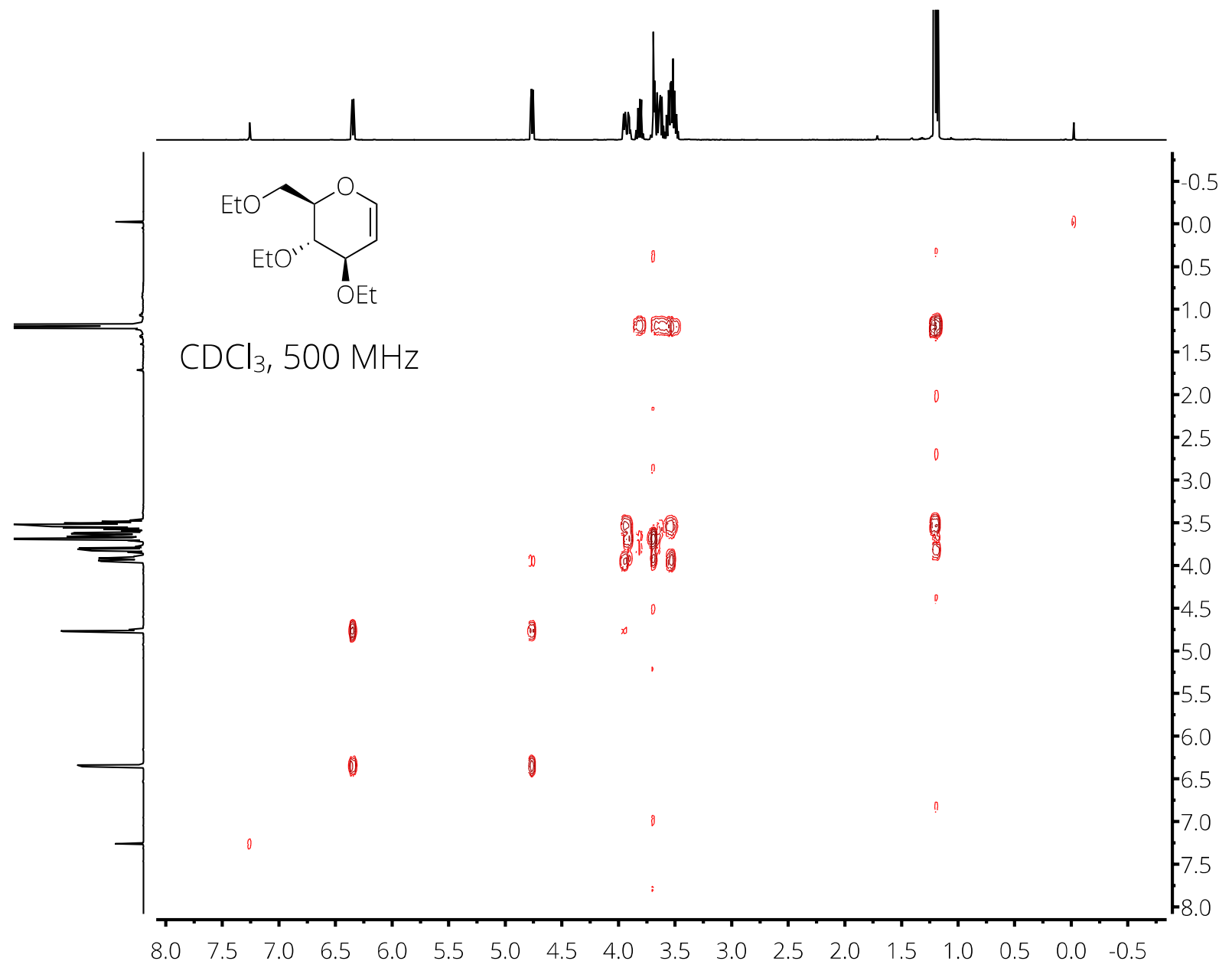




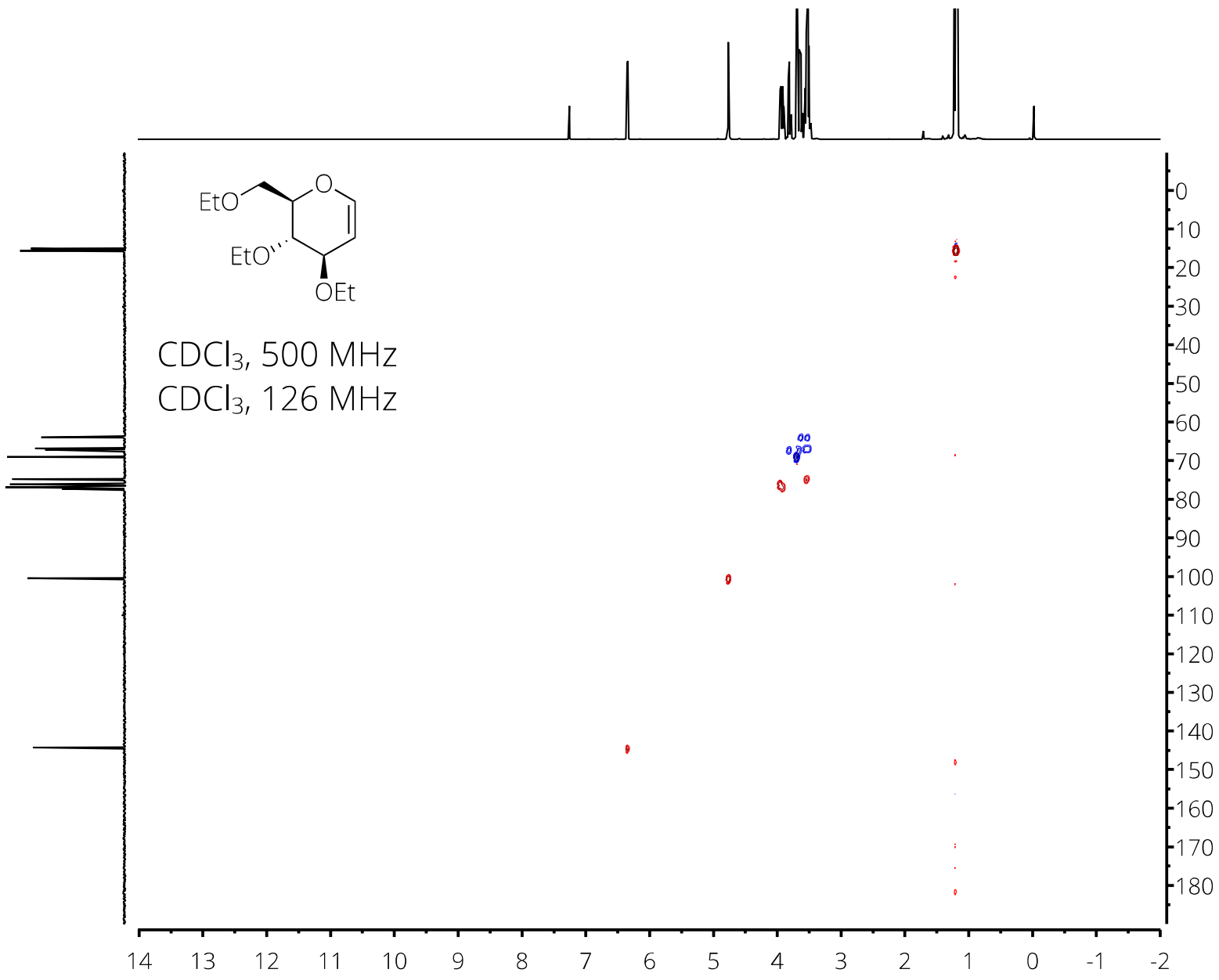


3,4,6-tri-O-triethylsilyl-D-glucal (5) - ${ }^{1} \mathrm{H}-\mathrm{NMR}$

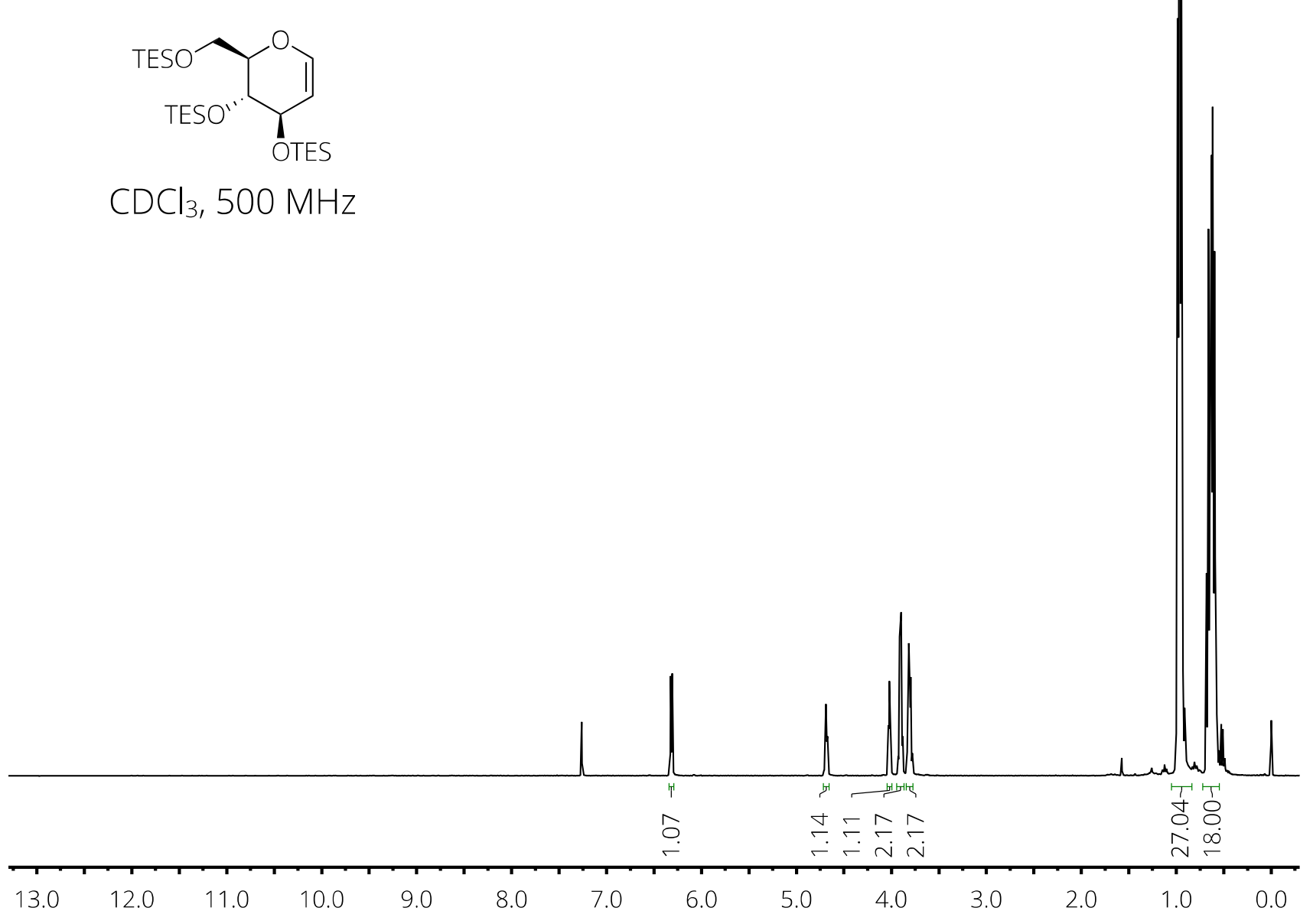

S

41 
3,4,6-tri-O-triethylsilyl-D-glucal (5) - ${ }^{13} \mathrm{C}\left\{{ }^{1} \mathrm{H}\right\}-\mathrm{NMR}$

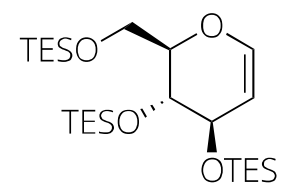

$\mathrm{CDCl}_{3}, 126 \mathrm{MHz}$

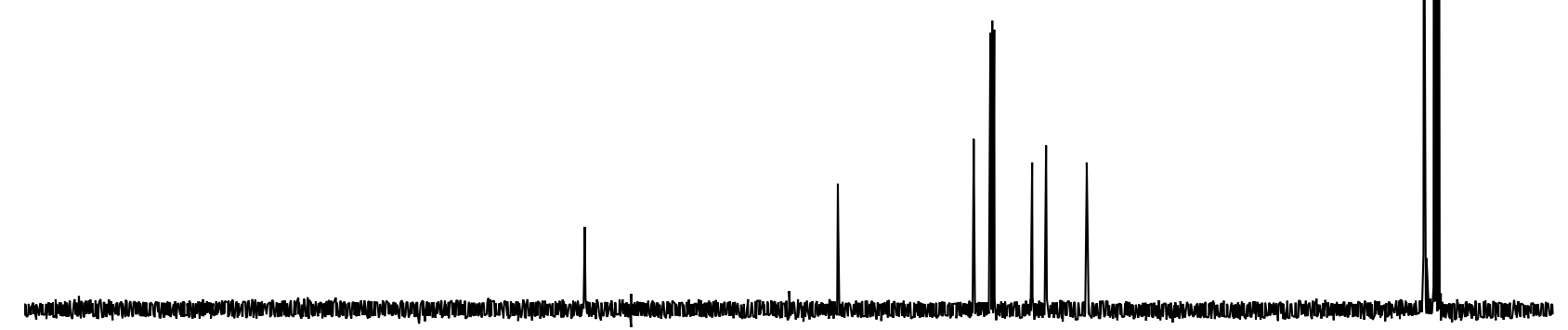

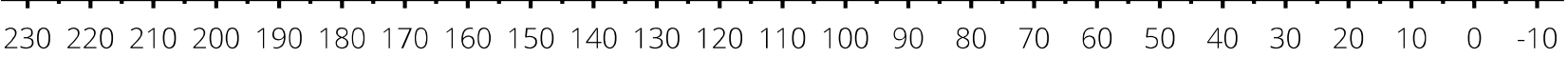


3,4,6-tri-O-triethylsilyl-D-glucal (5) - ${ }^{1} \mathrm{H}^{1} \mathrm{H}-\mathrm{COSY}$

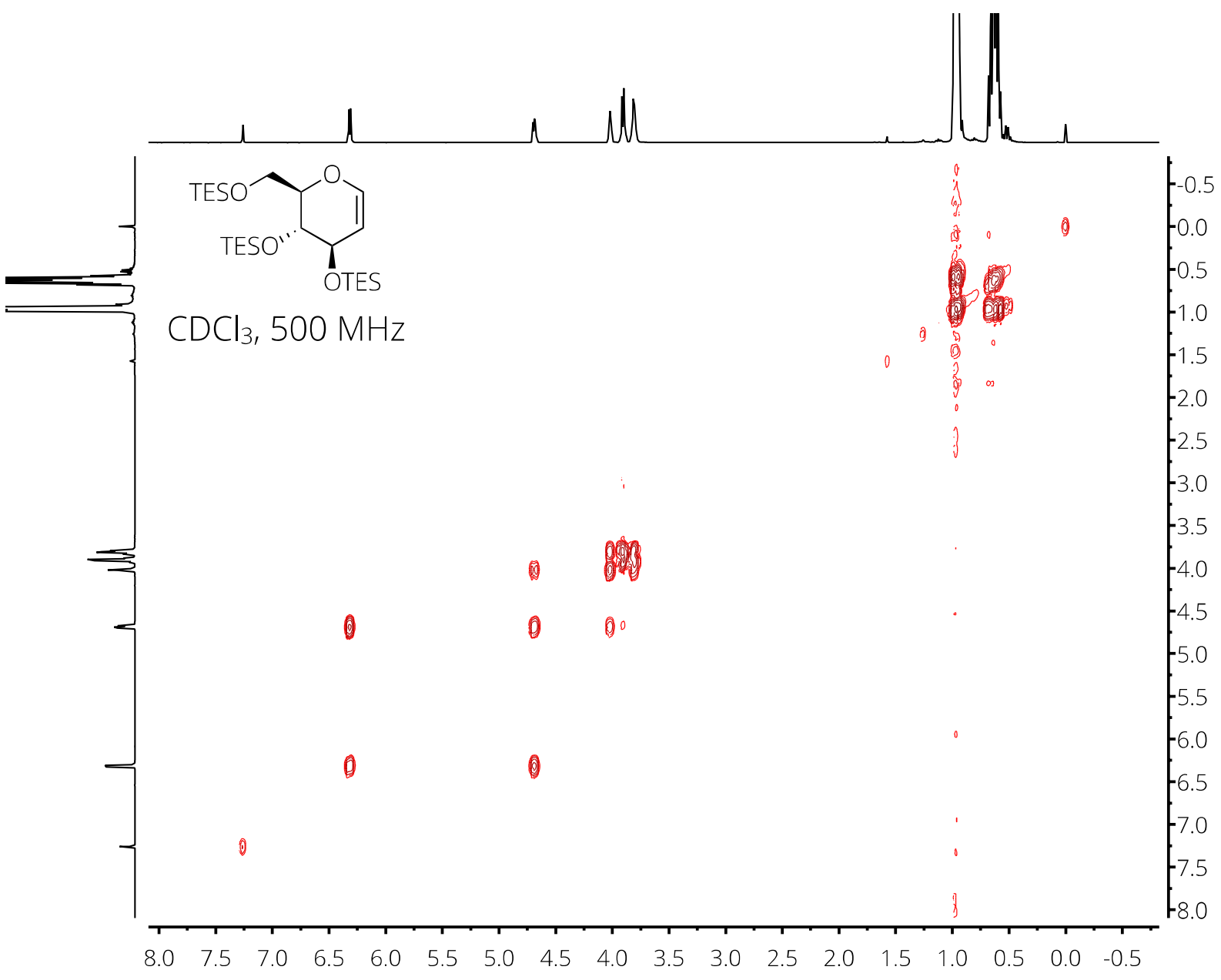

S

43 
3,4,6-tri-O-triethylsilyl-D-glucal (5) $-{ }^{1} \mathrm{H}^{13} \mathrm{C}\left\{{ }^{1} \mathrm{H}\right\}$-HSQC

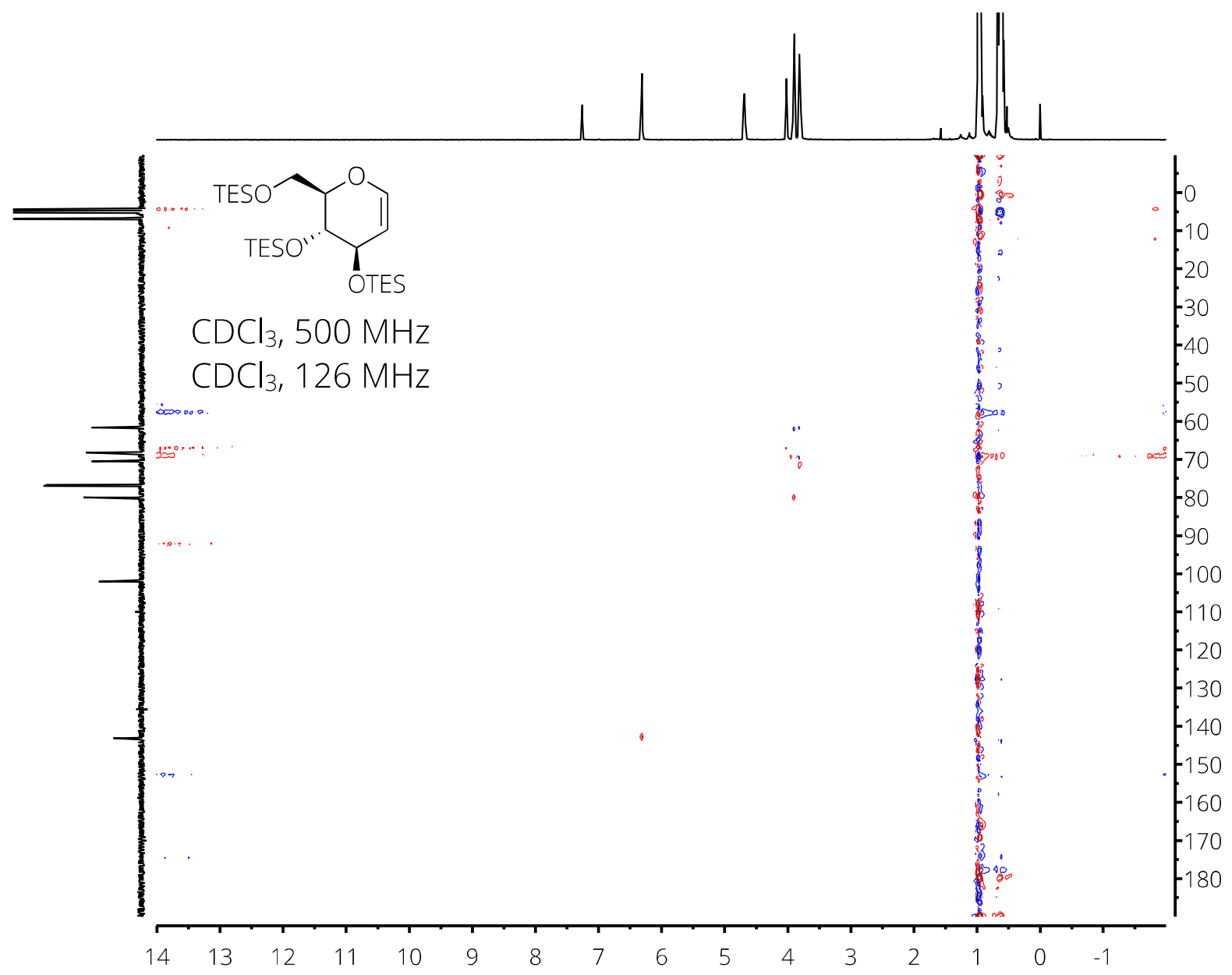

S

44 
3,4,6-tri-O-(p-methoxybenzyl)-D-glucal (6) - ${ }^{1} \mathrm{H}-\mathrm{NMR}$

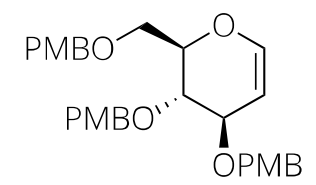

$\mathrm{CDCl}_{3}, 500 \mathrm{MHz}$

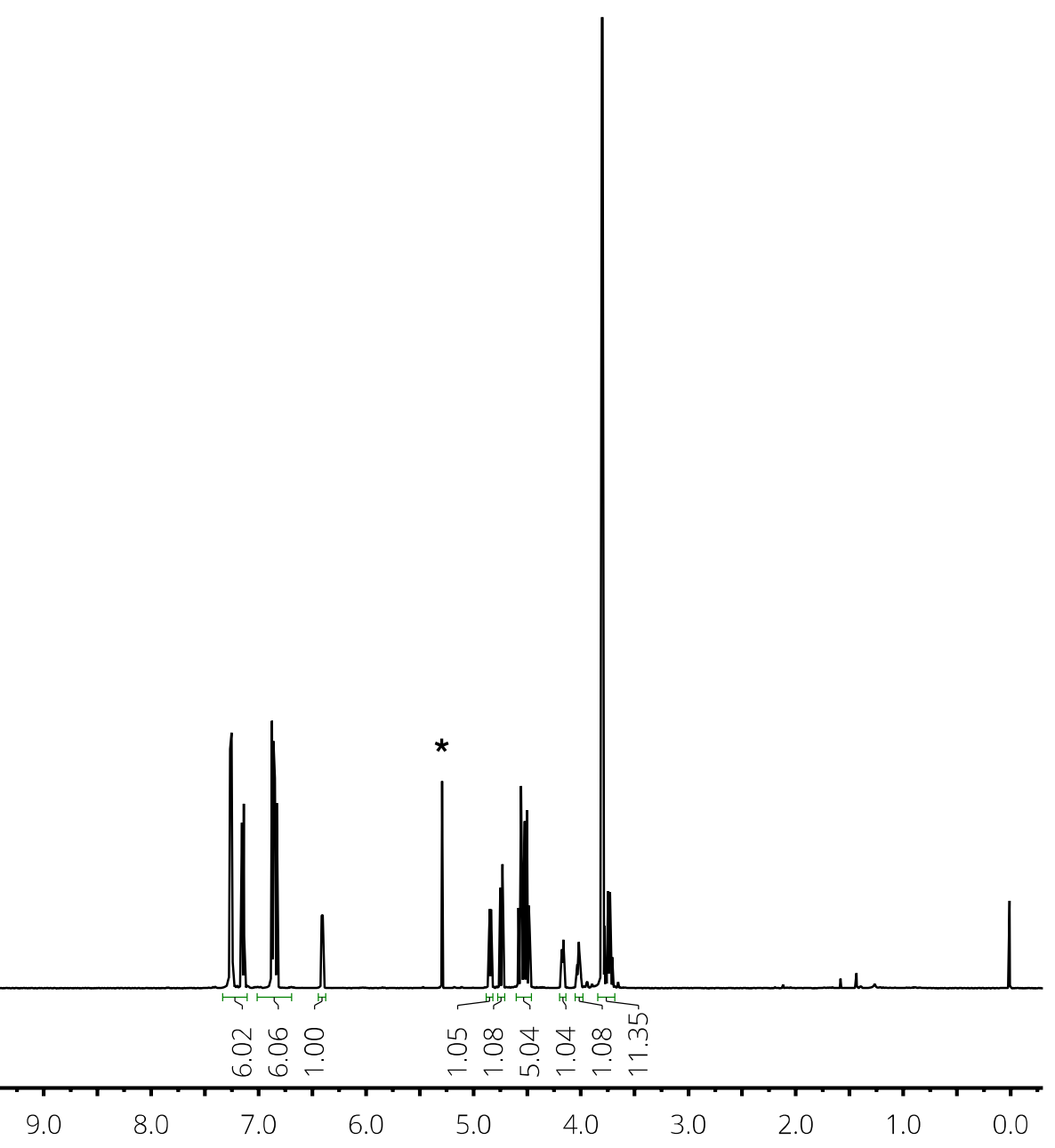

$*=$ solvent $(\mathrm{DCM})$ 


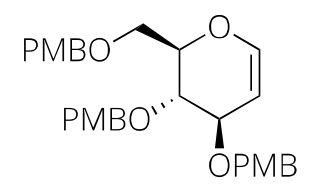

$\mathrm{CDCl}_{3}, 126 \mathrm{MHz}$

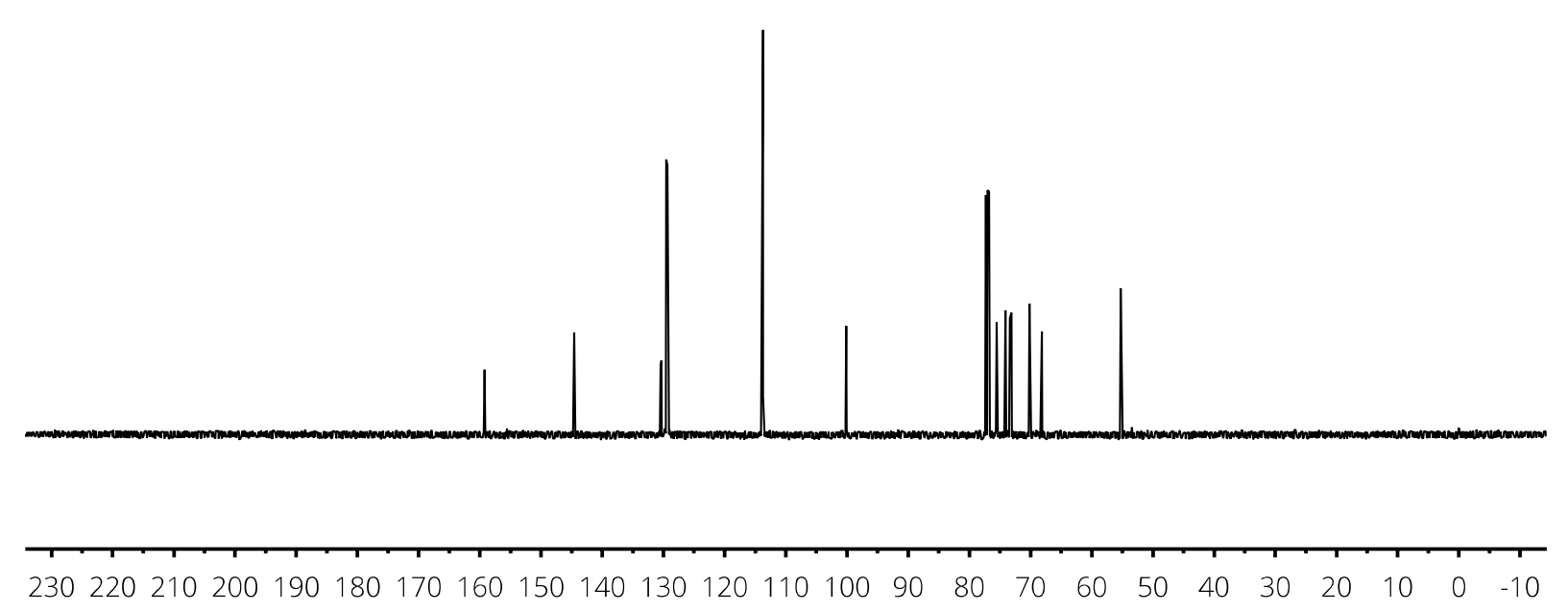


3,4,6-tri-O-(p-methoxybenzyl)-D-glucal (6) - ${ }^{1} \mathrm{H}^{1} \mathrm{H}-\mathrm{COSY}$

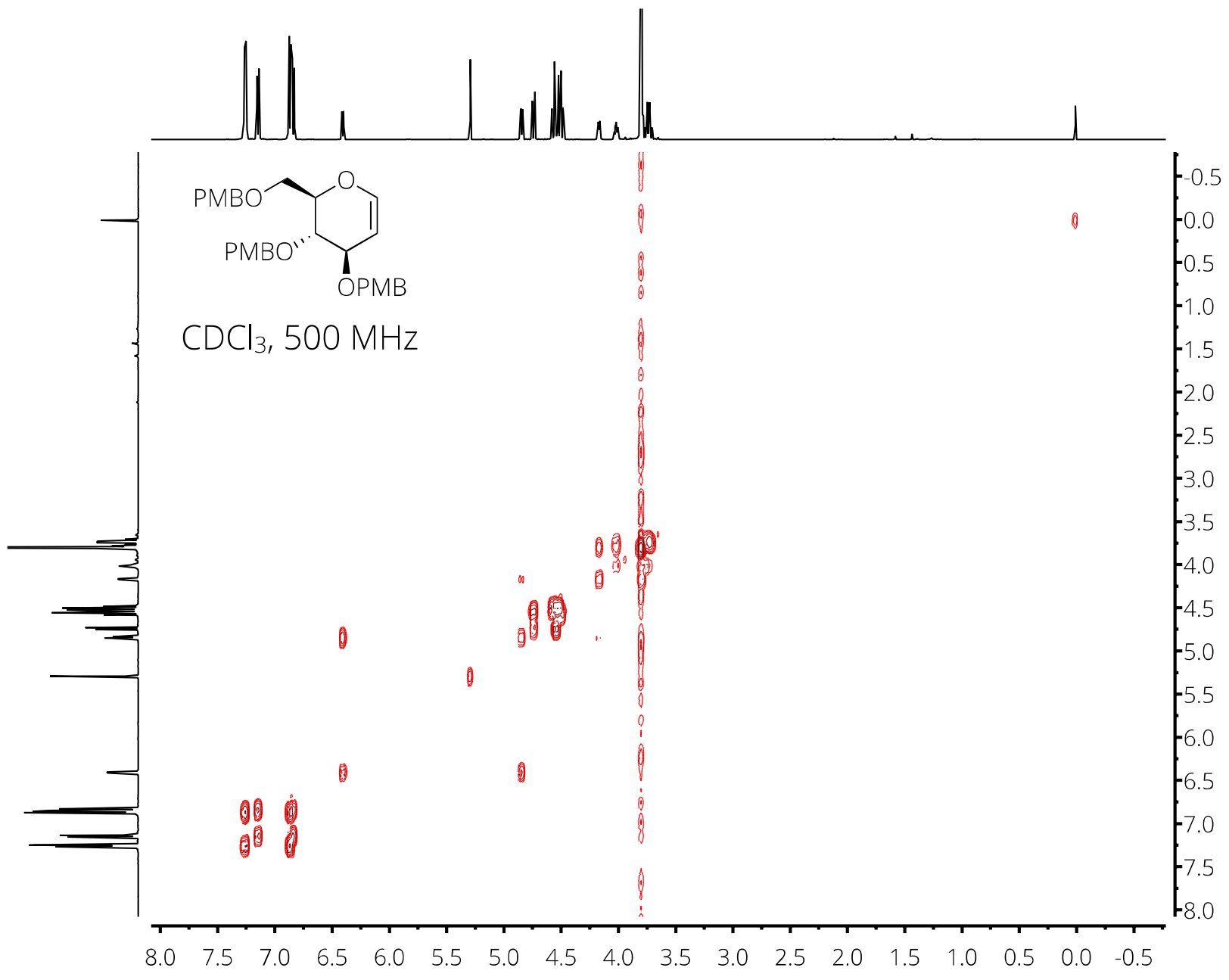

S

47 


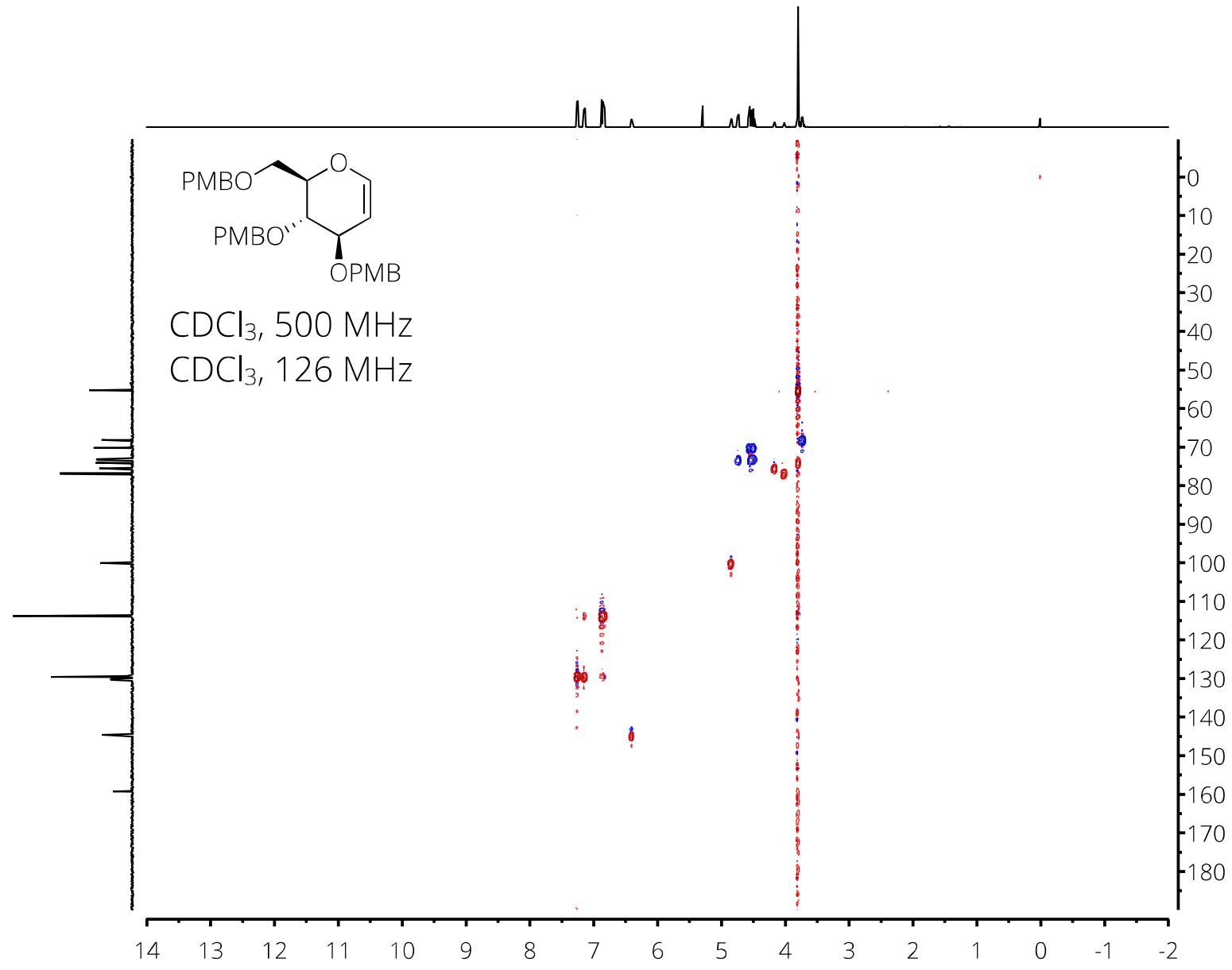


3,4,6-tri-O-(2,2,2-trichloroethylcarbonate)-D-glucal (7) - ${ }^{1} \mathrm{H}-\mathrm{NMR}$

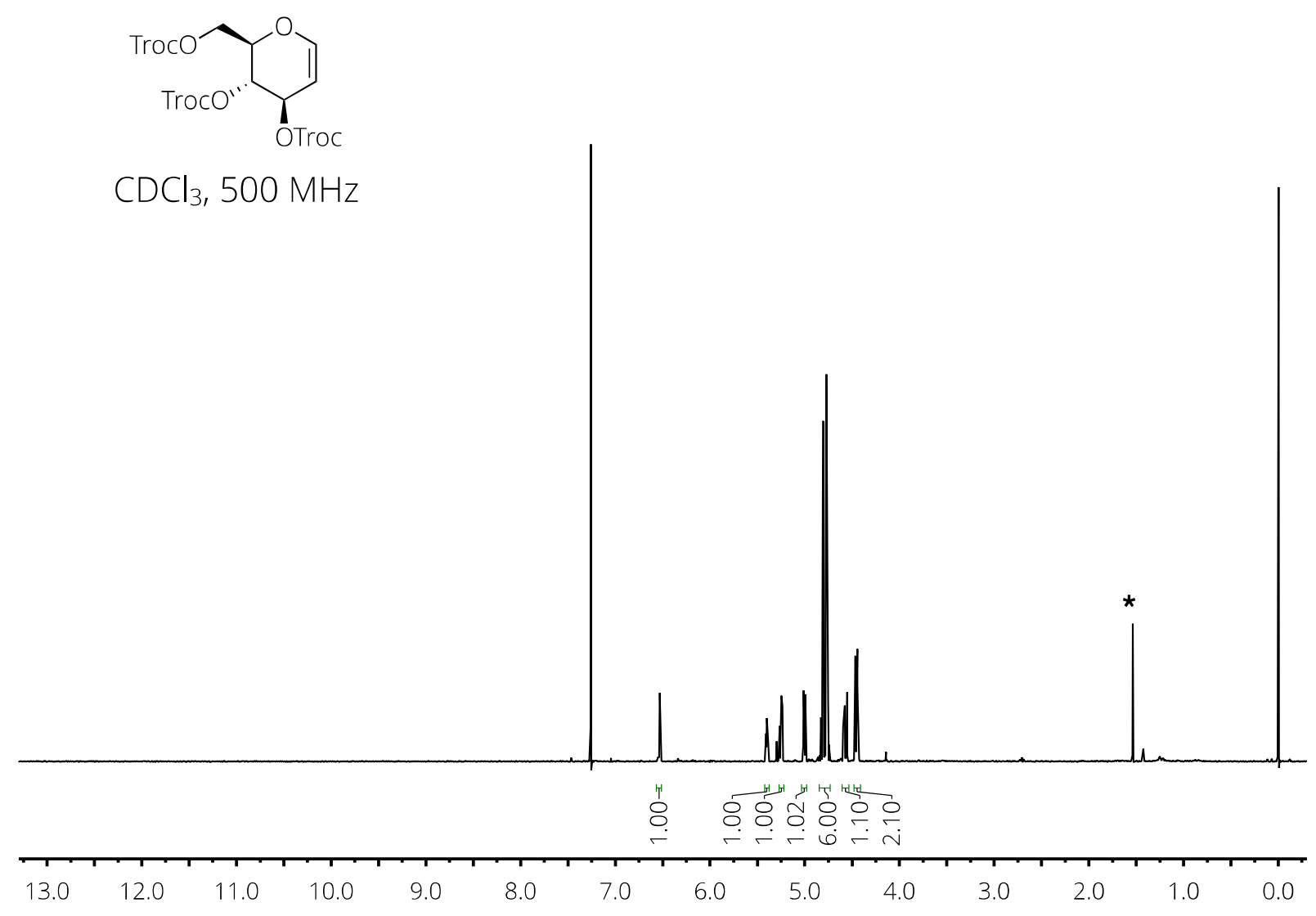

$*$ = solvent (cyclohexane) 
3,4,6-tri-O-(2,2,2-trichloroethylcarbonate)-D-glucal (7) - ${ }^{13} \mathrm{C}\left\{{ }^{1} \mathrm{H}\right\}$-NMR

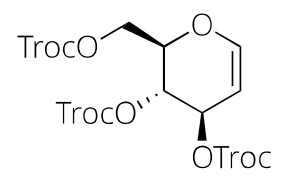

$\mathrm{CDCl}_{3}, 126 \mathrm{MHz}$

$230220210200190180170160150140130120110100 \quad 90 \quad 80 \quad 70 \quad 60 \begin{array}{llllllll}50 & 40 & 30 & 20 & 10 & 0 & -10\end{array}$ 
3,4,6-tri-O-(2,2,2-trichloroethylcarbonate)-D-glucal (7) - ${ }^{1} \mathrm{H}^{1} \mathrm{H}-\mathrm{COSY}$

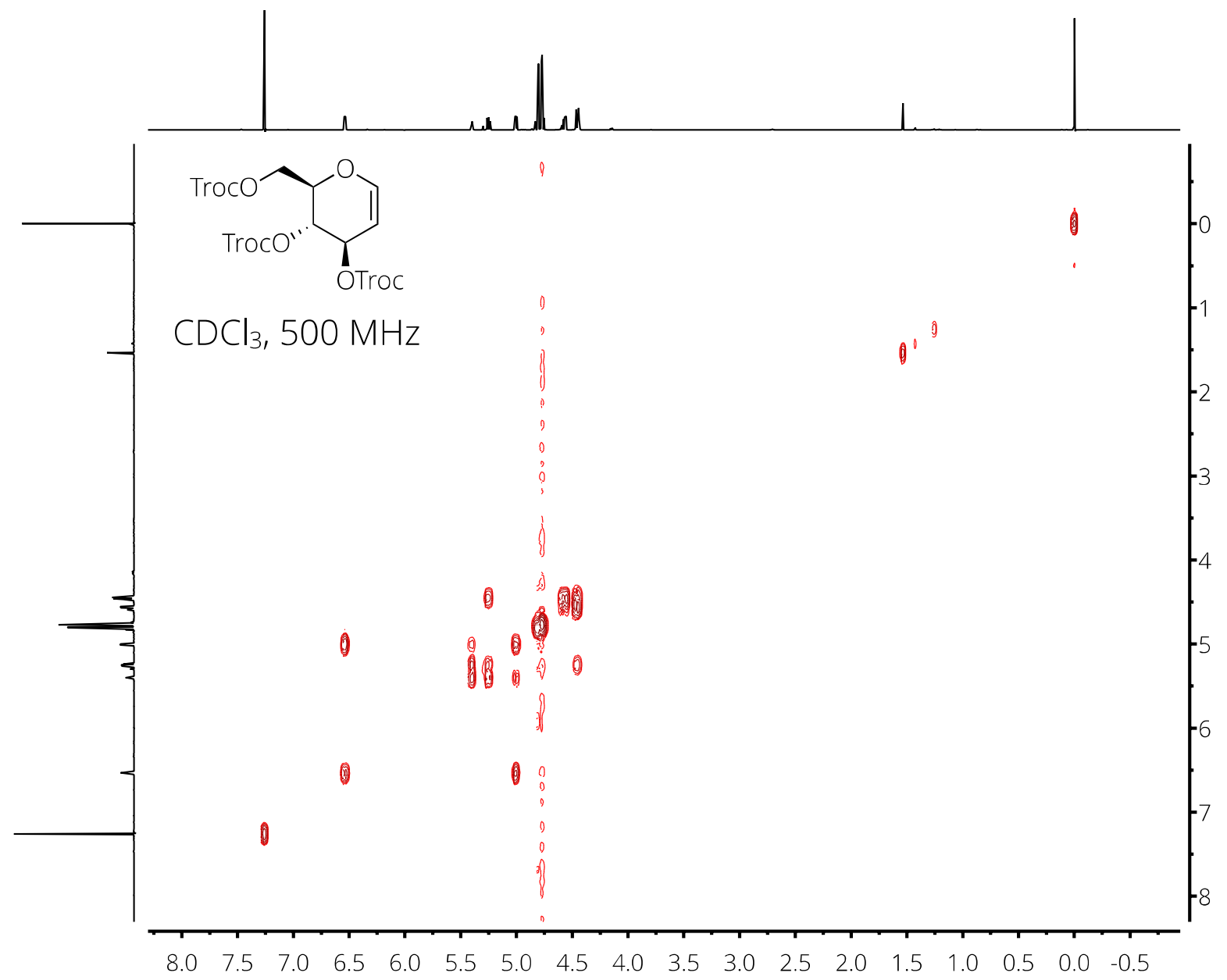


3,4,6-tri-O-(2,2,2-trichloroethylcarbonate)-D-glucal (7) - ${ }^{1} \mathrm{H}^{13} \mathrm{C}\left\{{ }^{1} \mathrm{H}\right\}$-HSQC

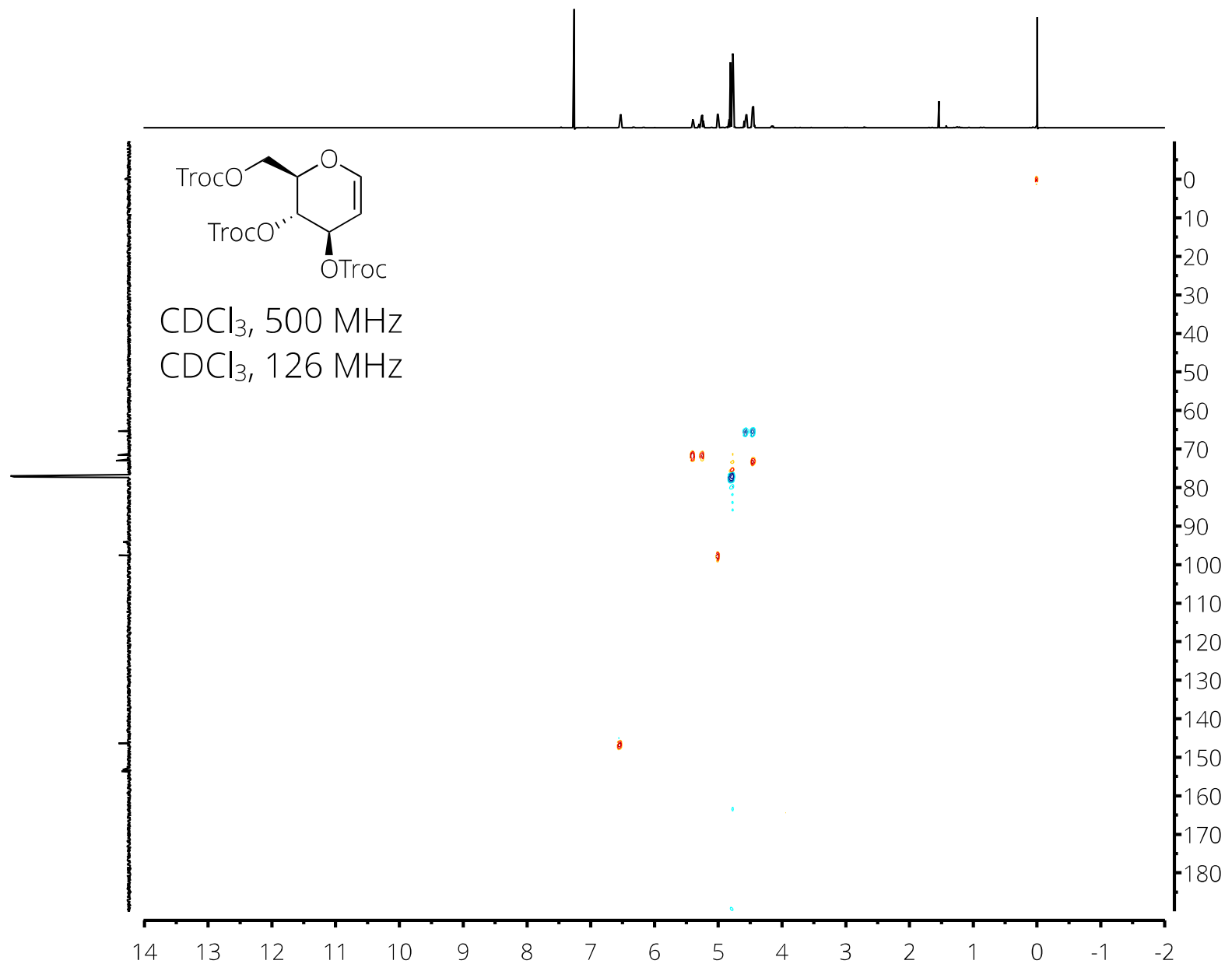

S

52 
3,4,6-tri-O-methoxymethyl-D-glucal (8) - ${ }^{1} \mathrm{H}-\mathrm{NMR}$

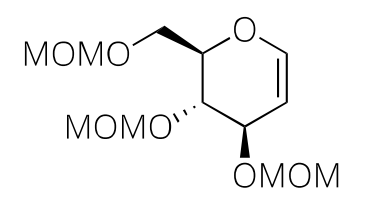

$\mathrm{CDCl}_{3}, 500 \mathrm{MHz}$

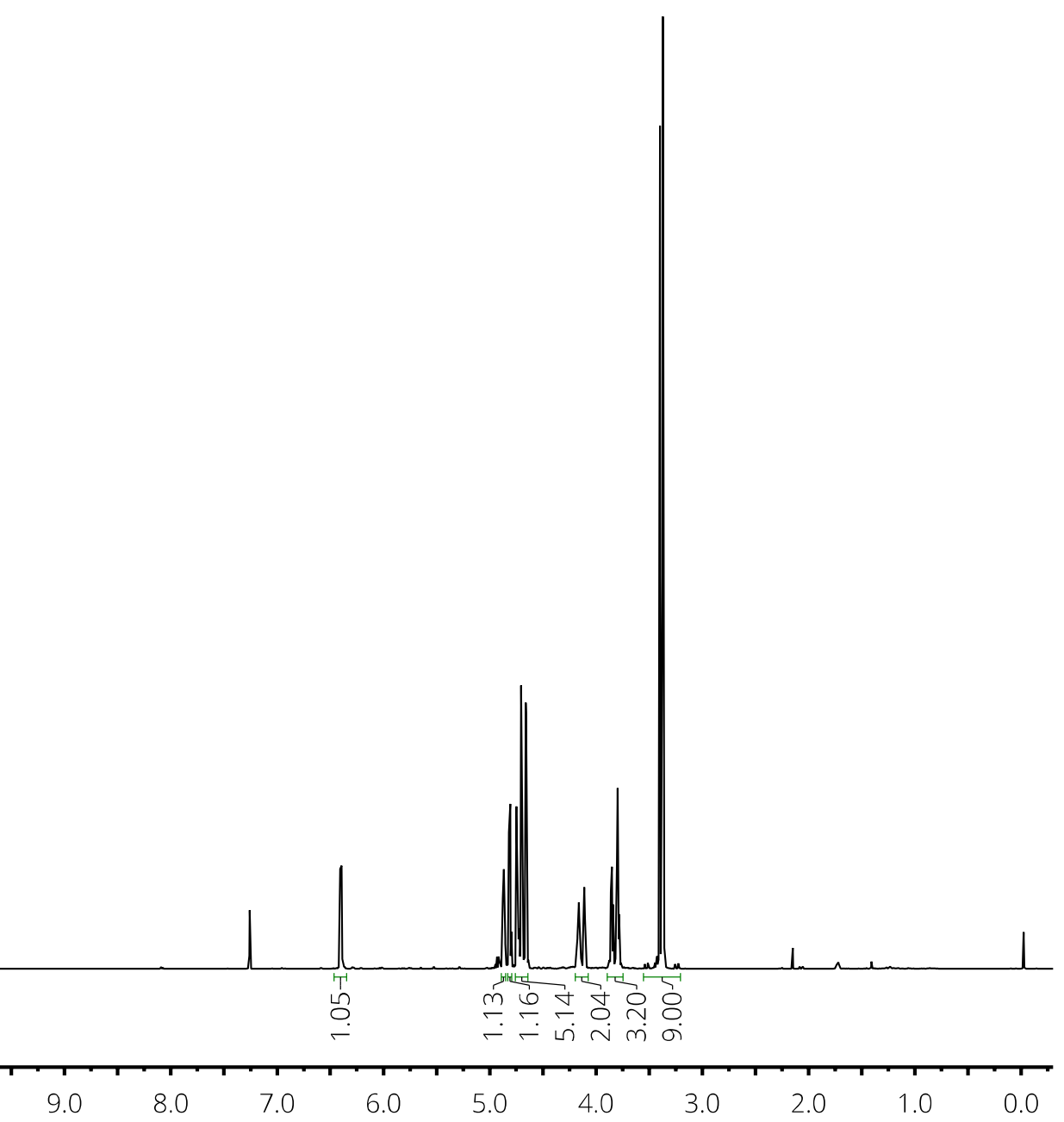




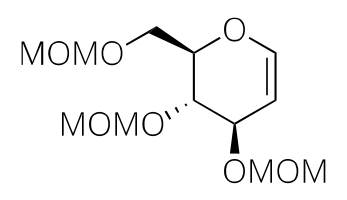

$\mathrm{CDCl}_{3}, 126 \mathrm{MHz}$
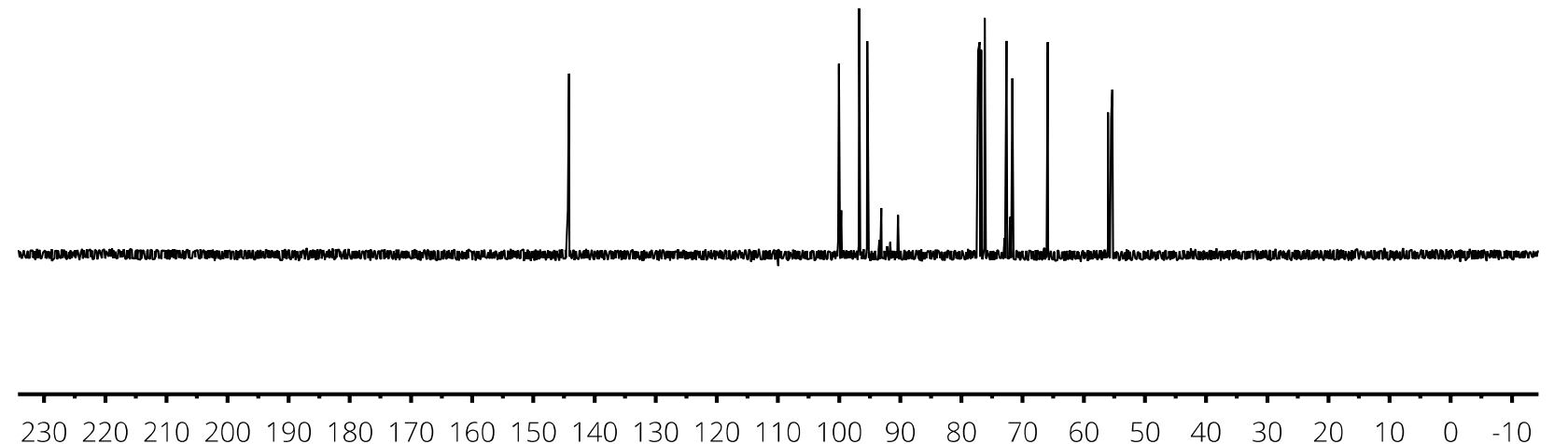


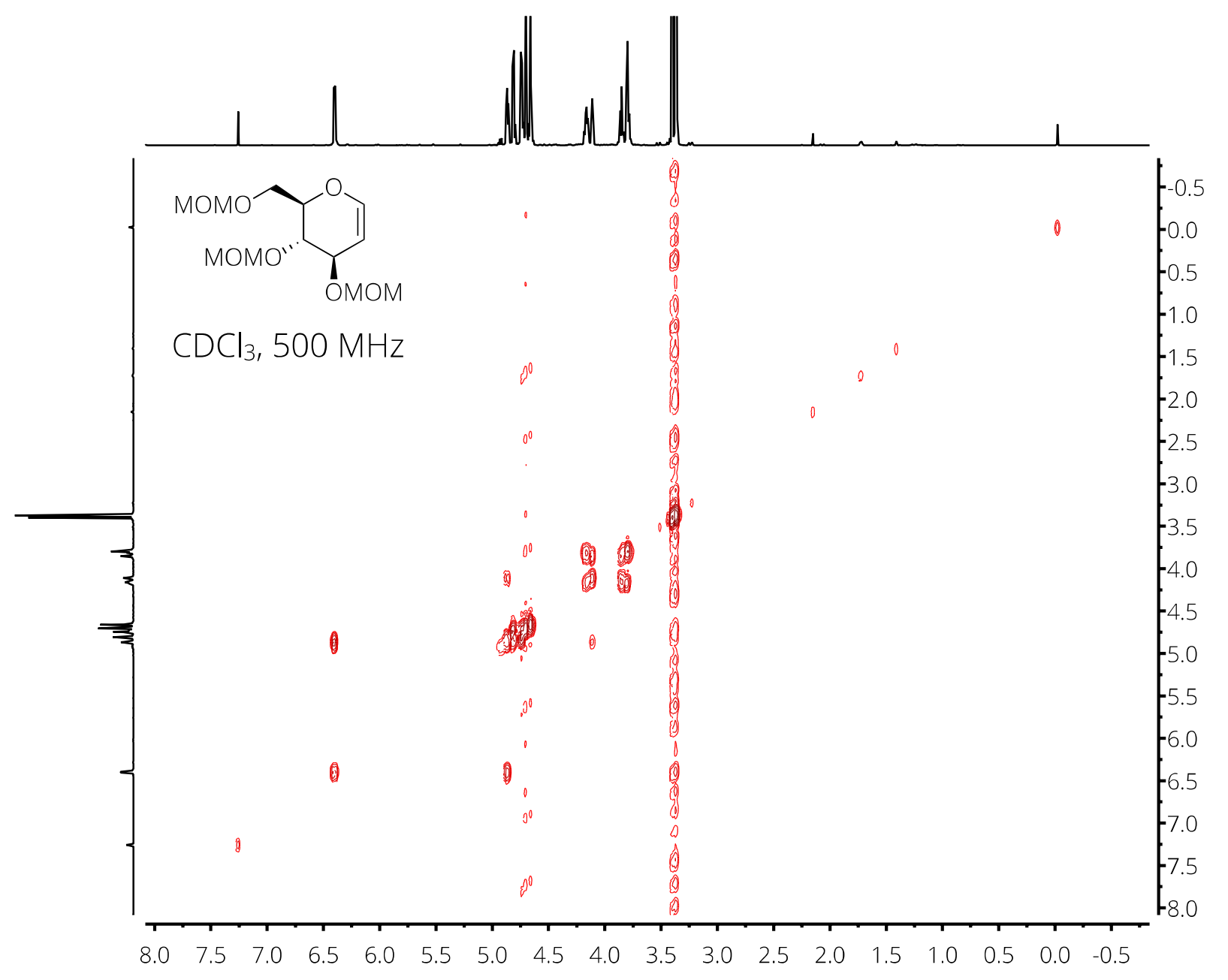


3,4,6-tri- $O$-methoxymethyl-D-glucal $(8)-{ }^{1} \mathrm{H}^{13} \mathrm{C}\left\{{ }^{1} \mathrm{H}\right\}$-HSQC

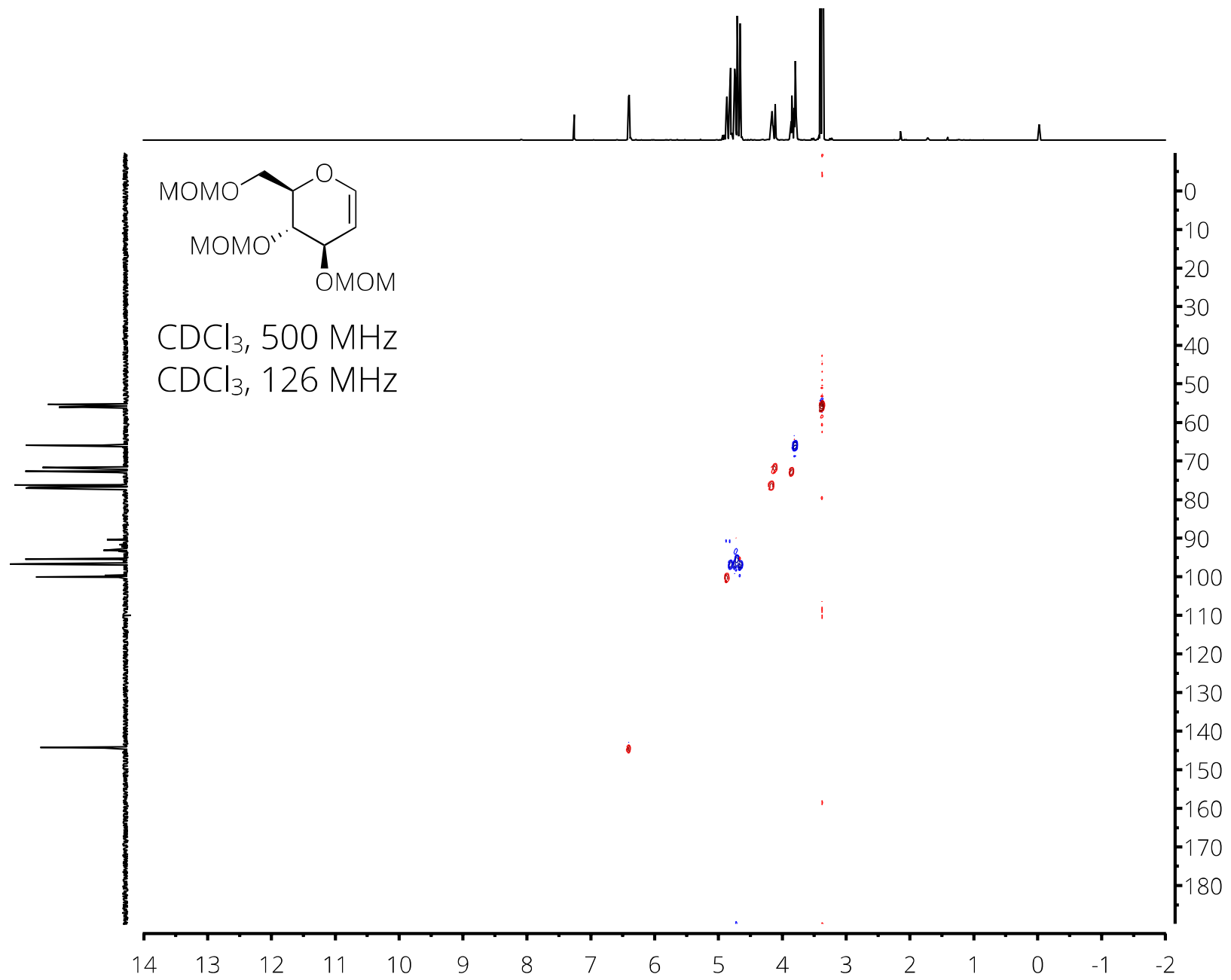




\section{Benzyl 3,4-di-O-benzyl-D-glucuronal (9) - ${ }^{1} \mathrm{H}-\mathrm{NMR}$}

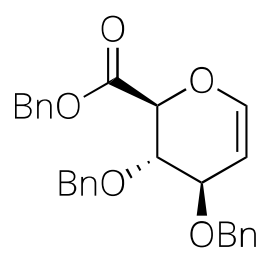

$\mathrm{CDCl}_{3}, 500 \mathrm{MHz}$

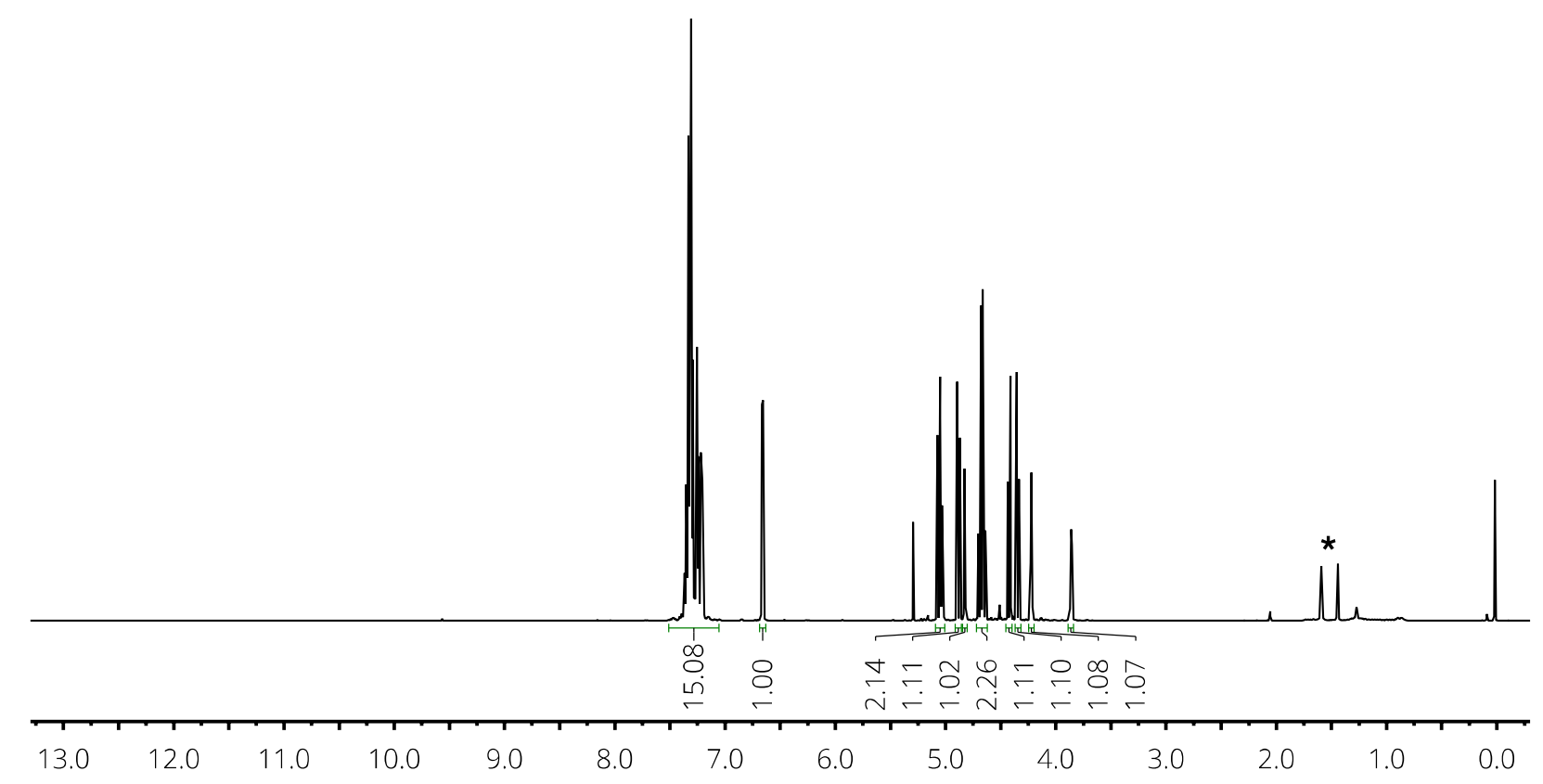

* = solvent (cyclohexane and DCM) 
Benzyl 3,4-di-O-benzyl-D-glucuronal (9) - ${ }^{13} \mathrm{C}\left\{{ }^{1} \mathrm{H}\right\}-\mathrm{NMR}$

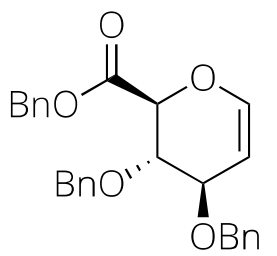

$\mathrm{CDCl}_{3}, 126 \mathrm{MHz}$

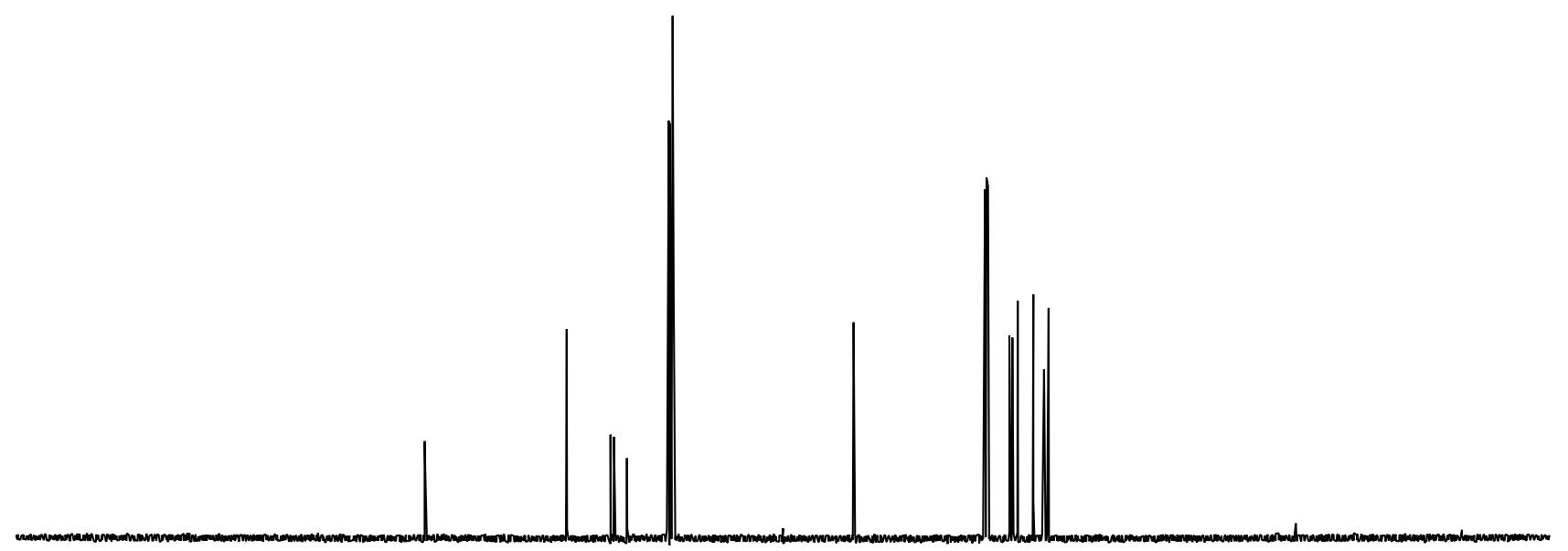

23022021020019018017016015014013012011010090 
Benzyl 3,4-di-O-benzyl-D-glucuronal (9) - ${ }^{1} \mathrm{H}^{1} \mathrm{H}-\mathrm{COSY}$

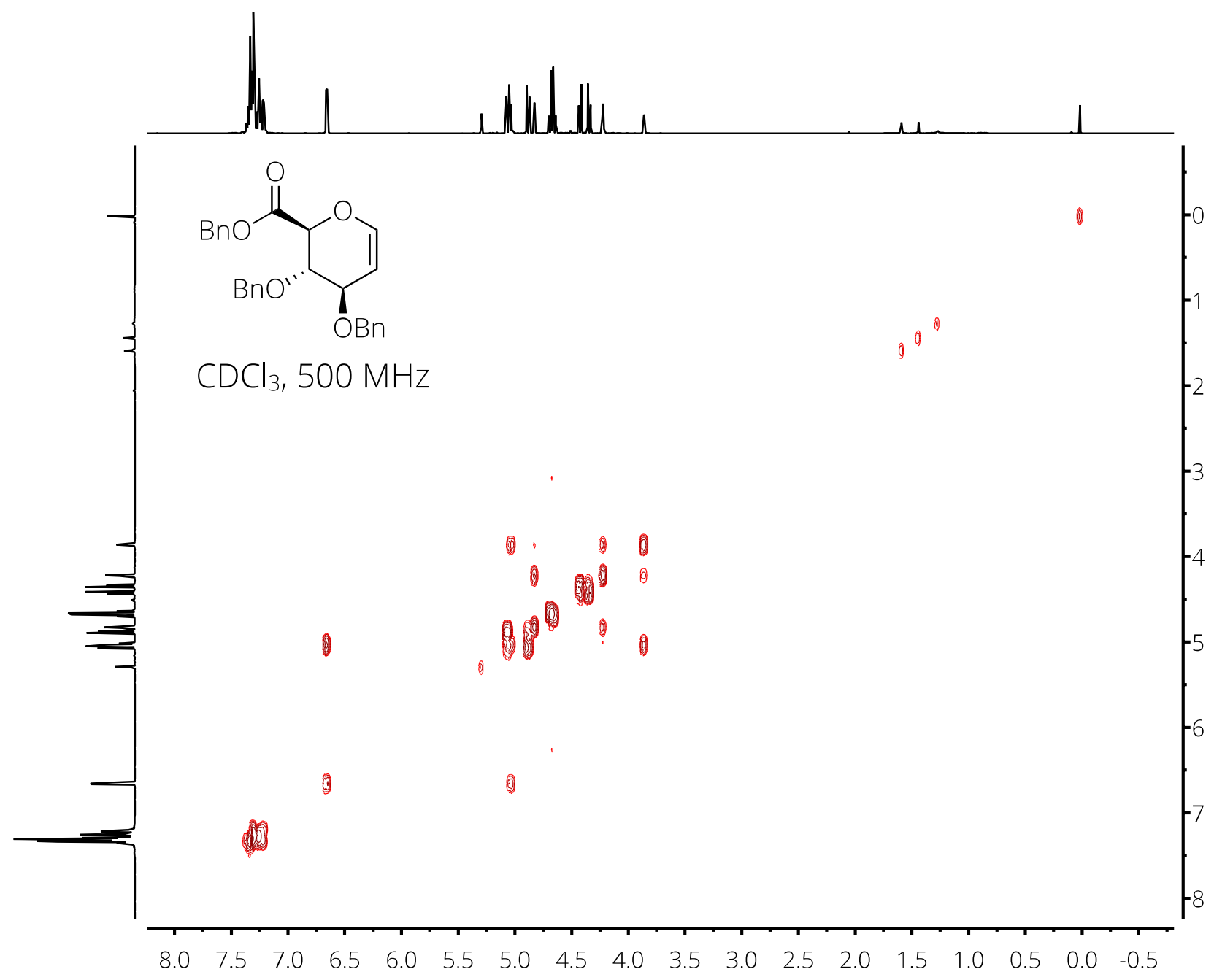


Benzyl 3,4-di-O-benzyl-D-glucuronal (9) - ${ }^{1} \mathrm{H}^{13} \mathrm{C}\left\{{ }^{1} \mathrm{H}\right\}$-HSQC

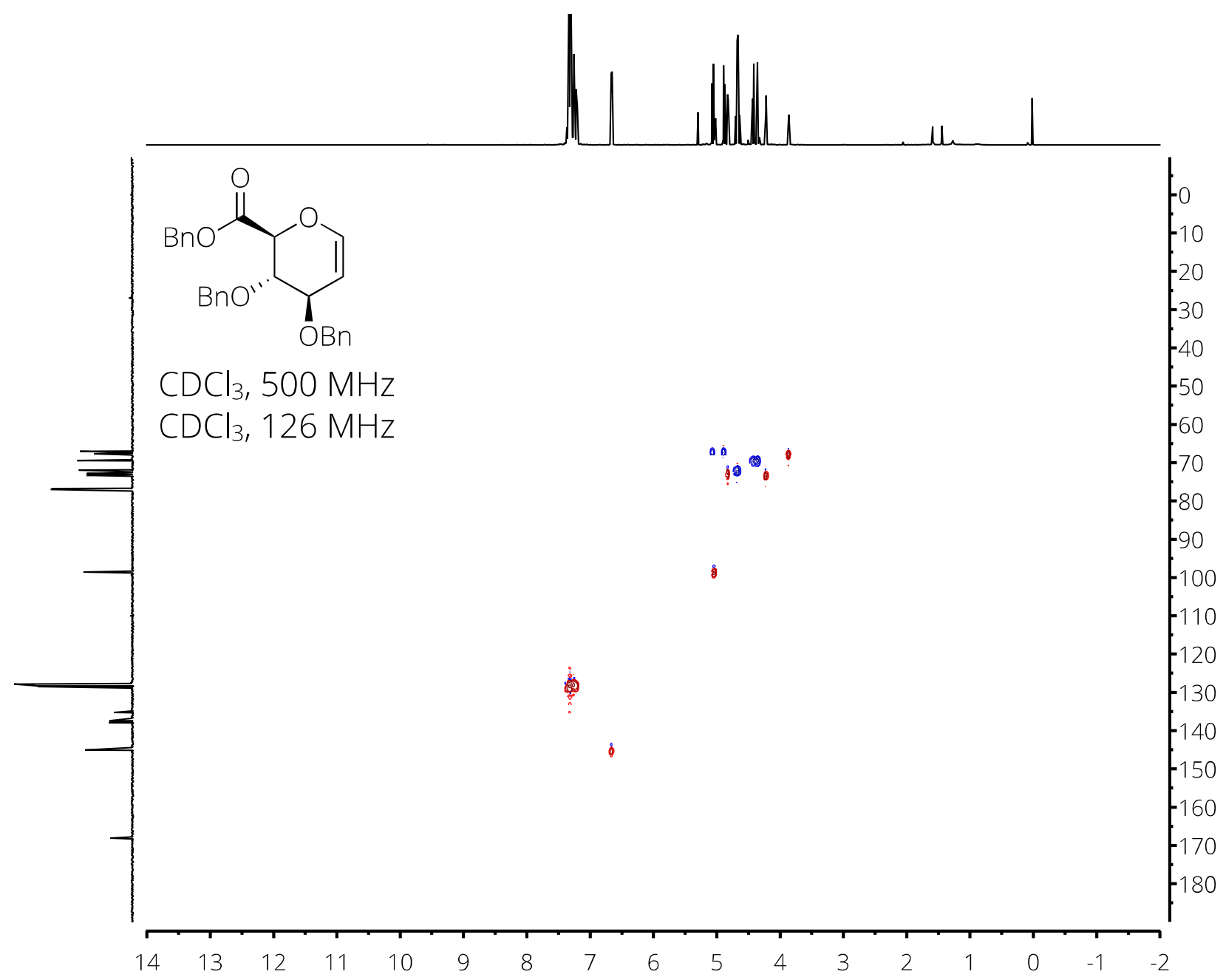


3,4-di-O-acetyl-6-O-triisopropylsilyl-D-glucal (10) - ${ }^{1} \mathrm{H}-\mathrm{NMR}$

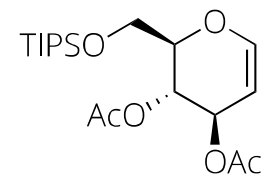

$\mathrm{CDCl}_{3}, 500 \mathrm{MHz}$

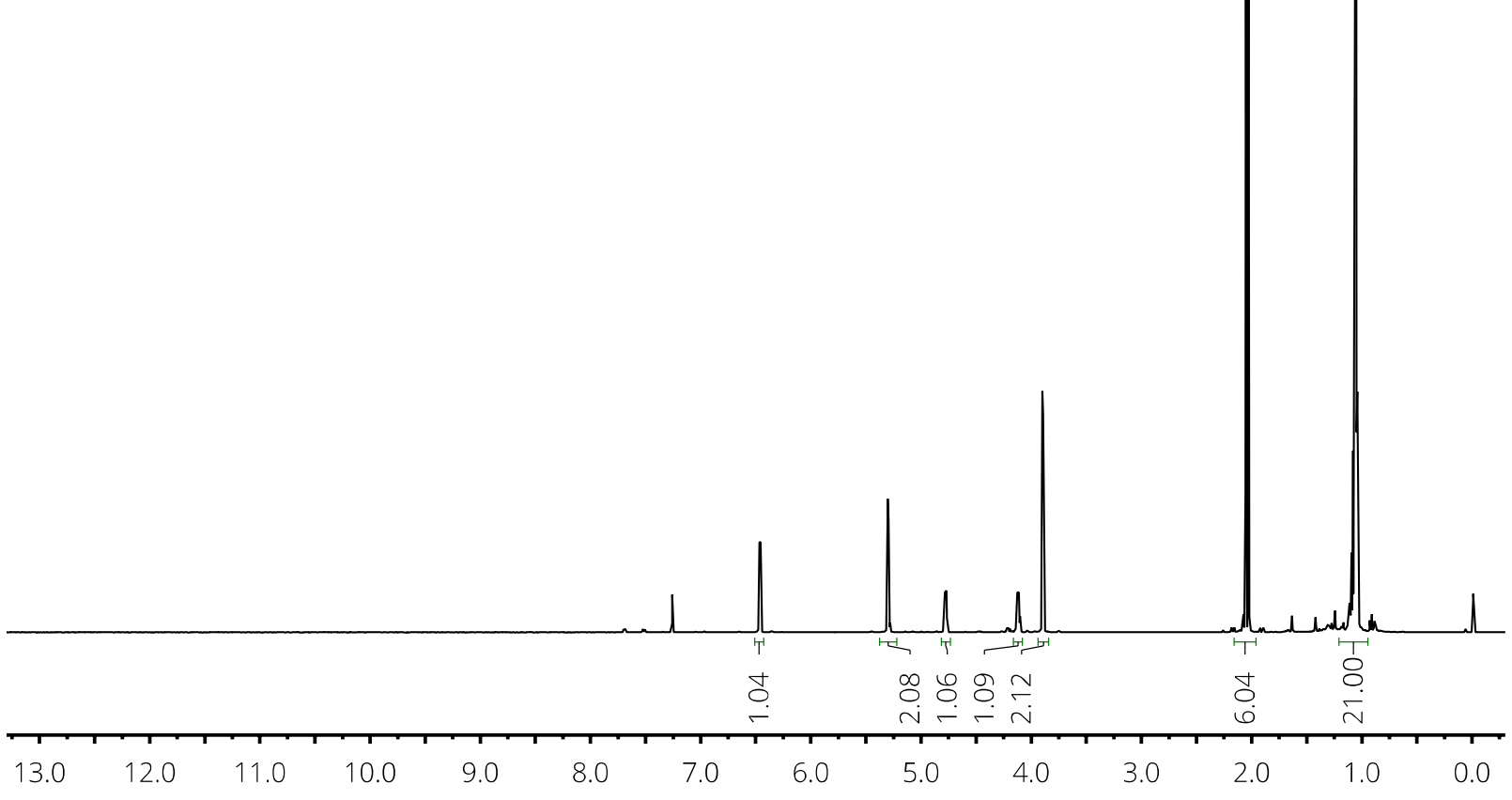


3,4-di- $O$-acetyl-6- $O$-triisopropylsilyl-D-glucal (10) - ${ }^{13} \mathrm{C}\left\{{ }^{1} \mathrm{H}\right\}$-NMR

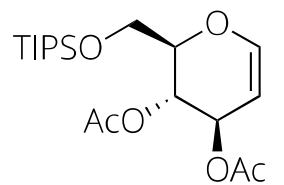

$\mathrm{CDCl}_{3}, 126 \mathrm{MHz}$
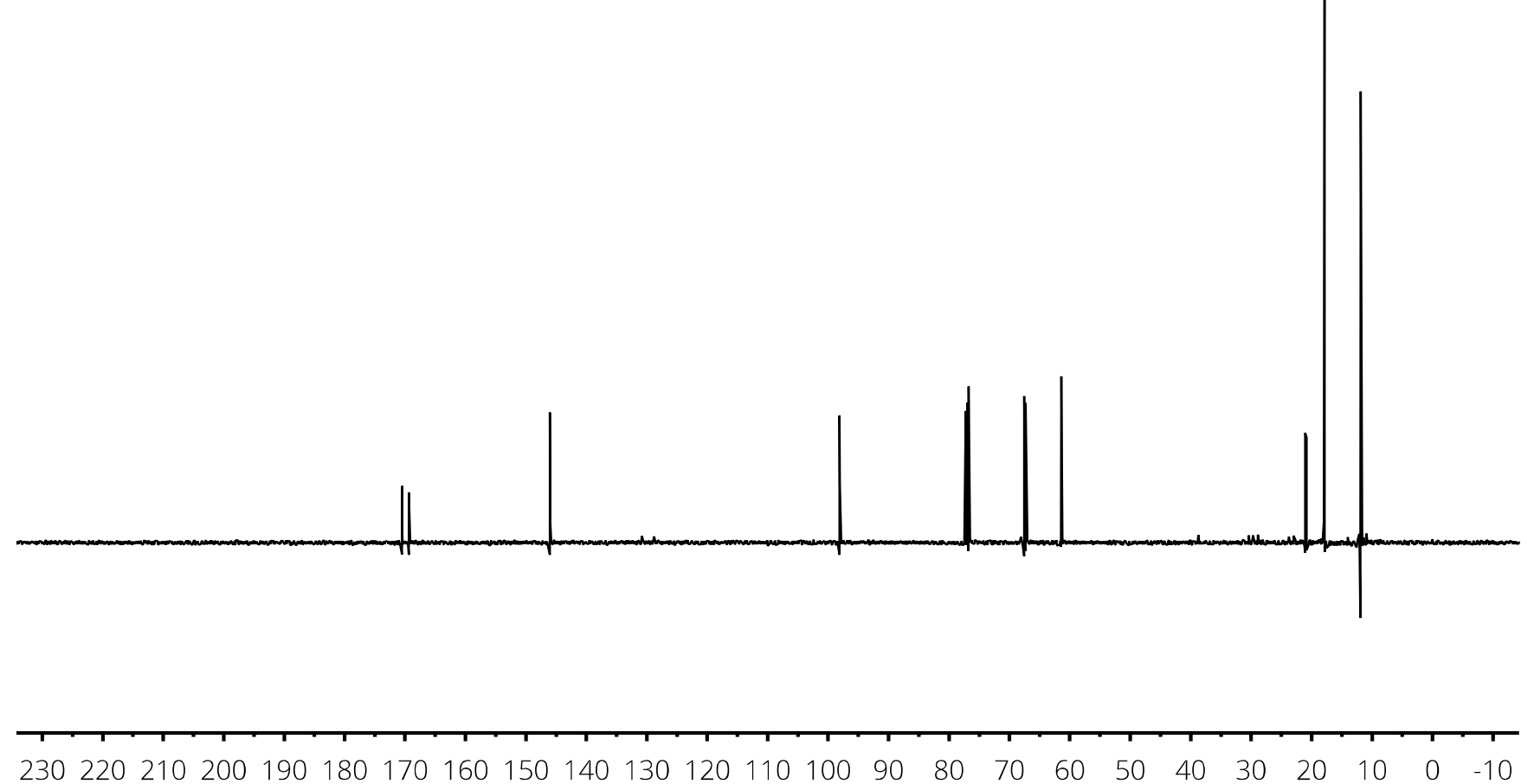


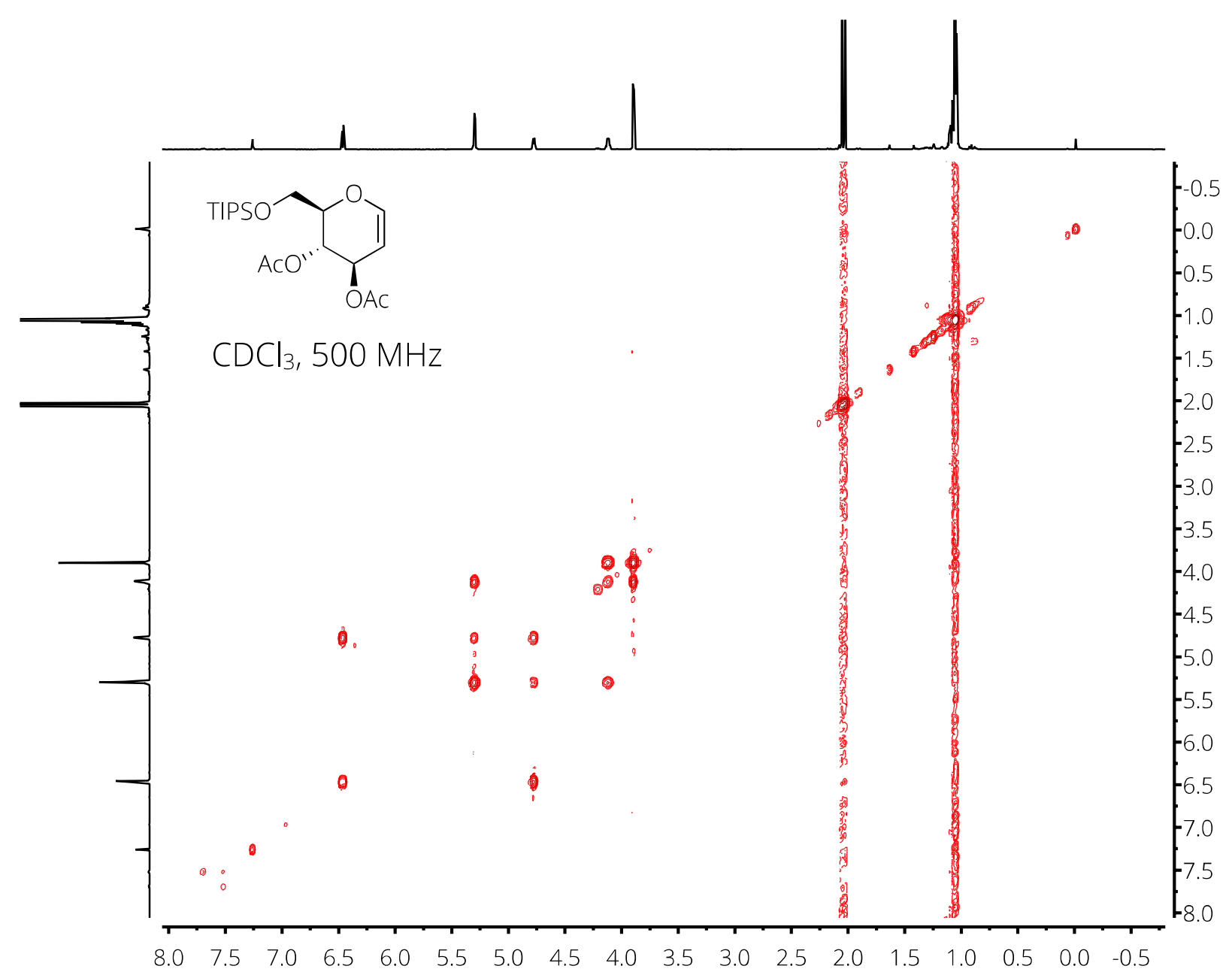




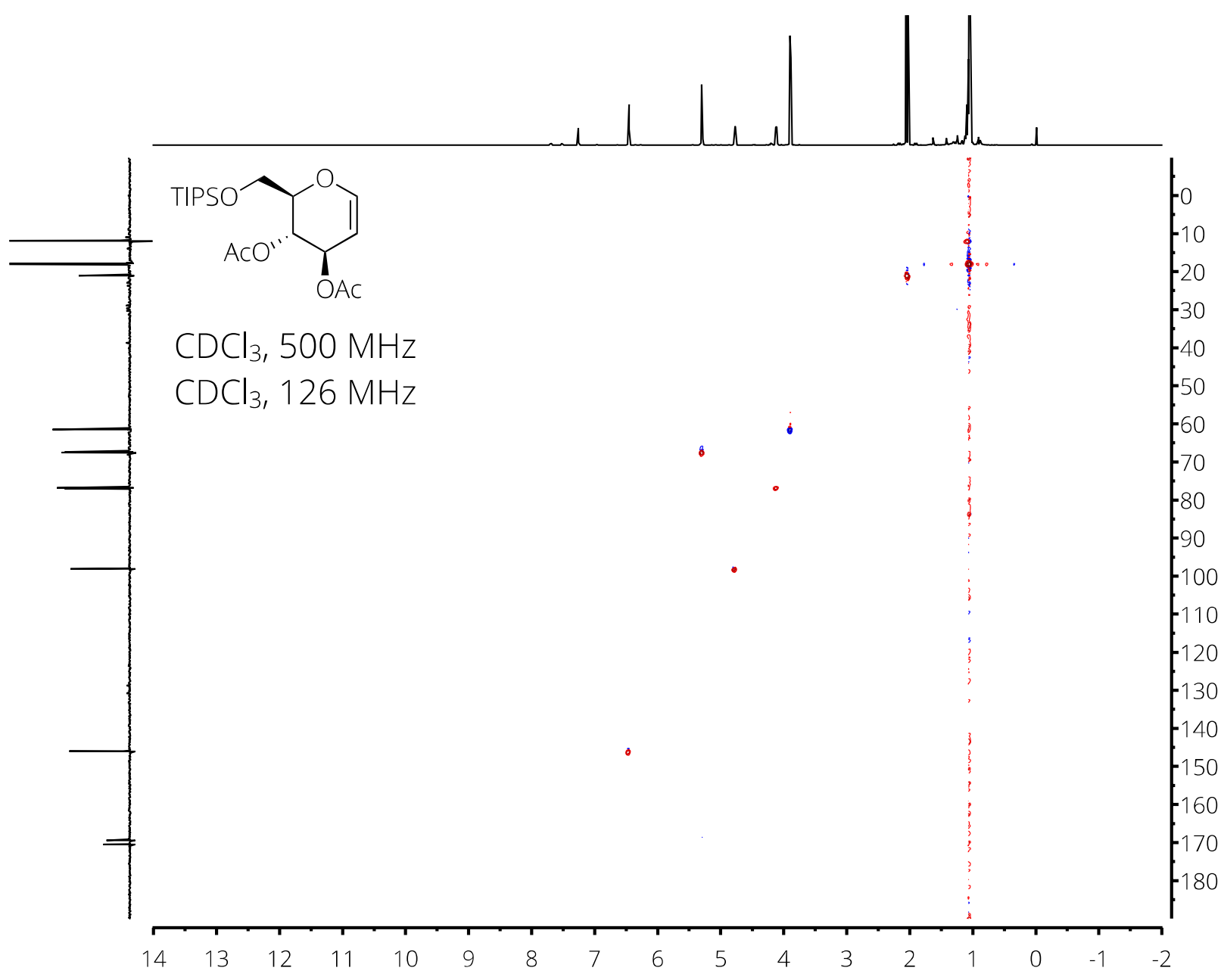




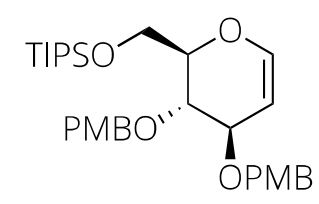

$\mathrm{CDCl}_{3}, 500 \mathrm{MHz}$

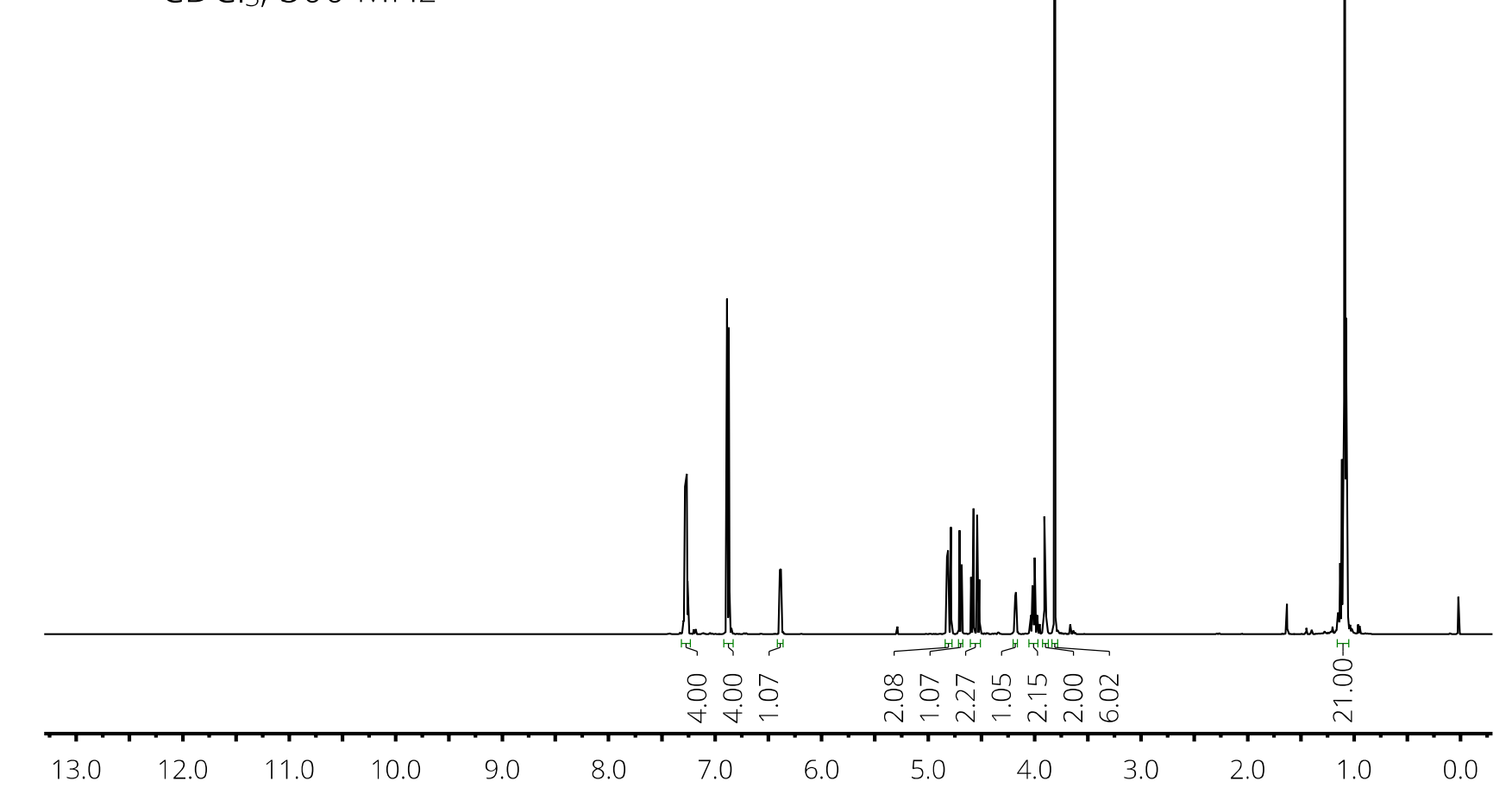




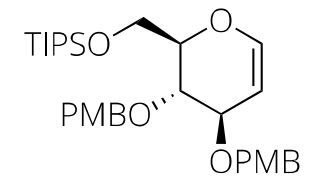

$\mathrm{CDCl}_{3}, 126 \mathrm{MHz}$

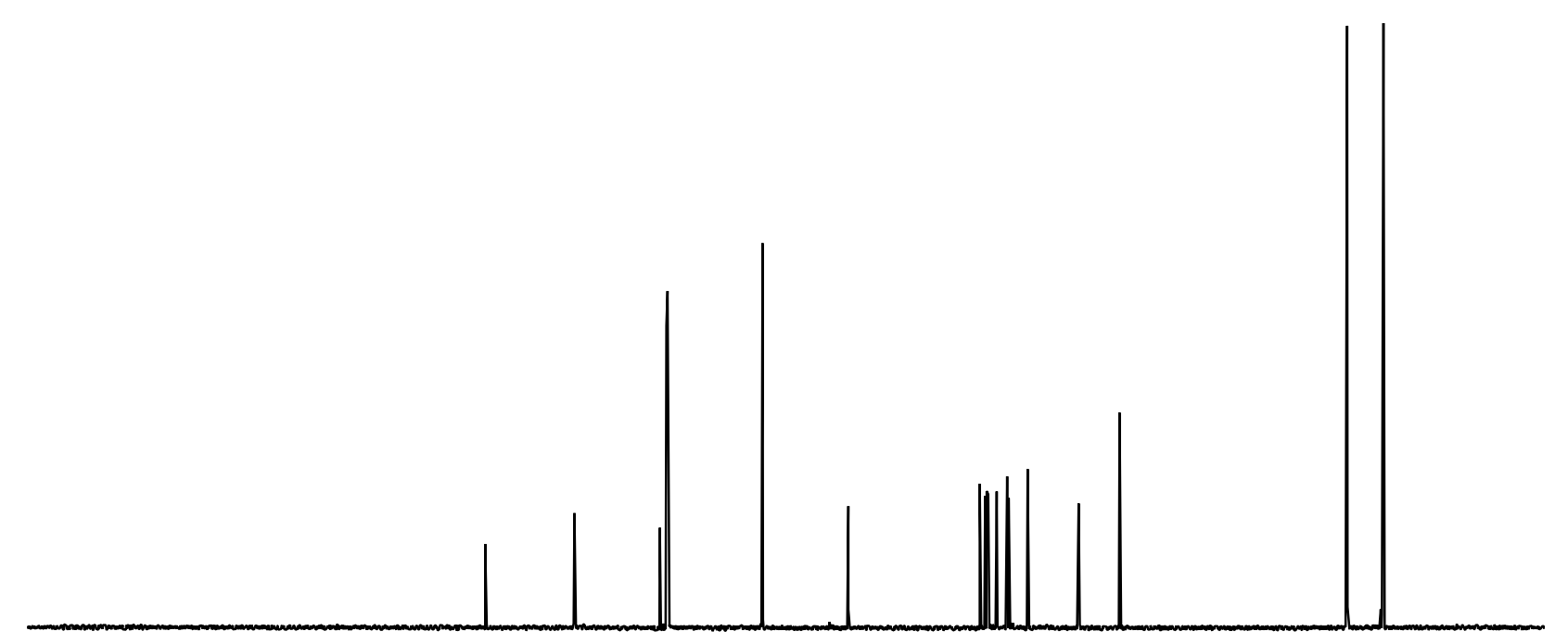

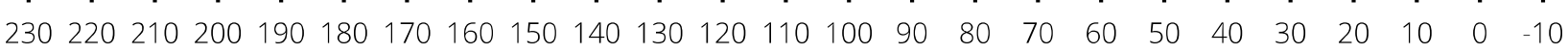




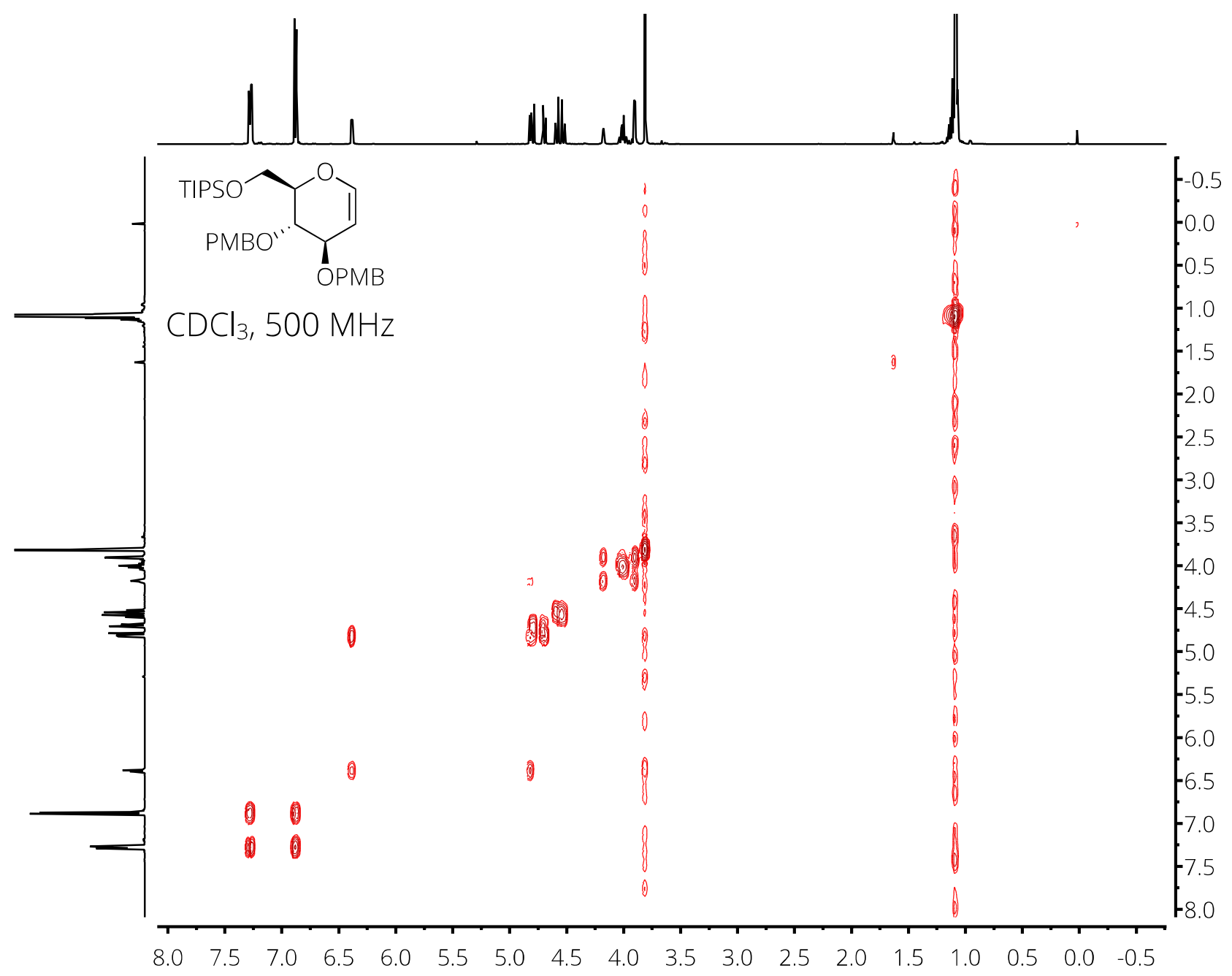




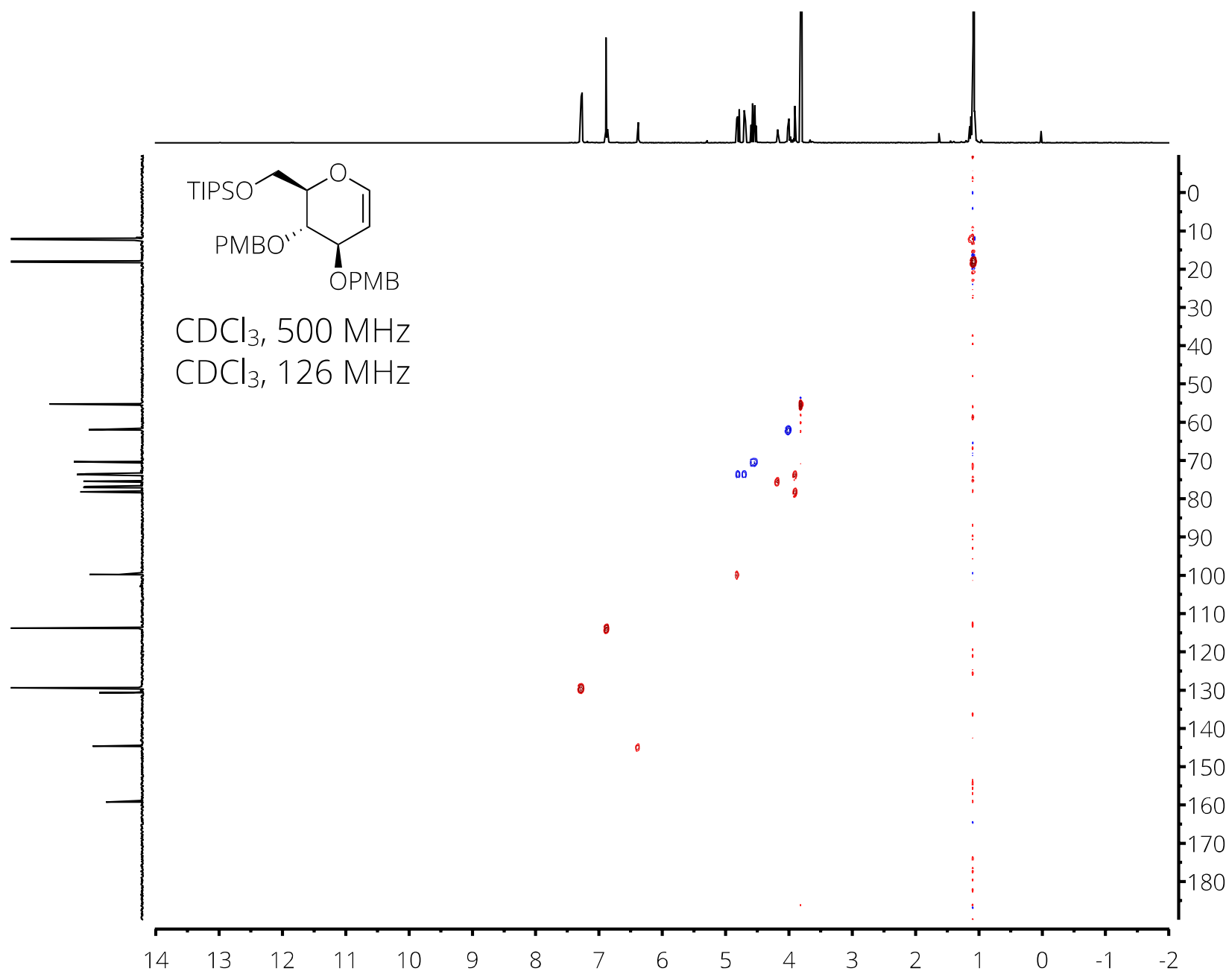


3,4-di-O-benzyl-6-O-triisopropylsilyl-D-glucal (12) - ${ }^{1} \mathrm{H}-\mathrm{NMR}$

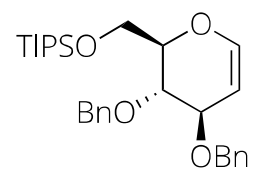

$\mathrm{CDCl}_{3}, 500 \mathrm{MHz}$

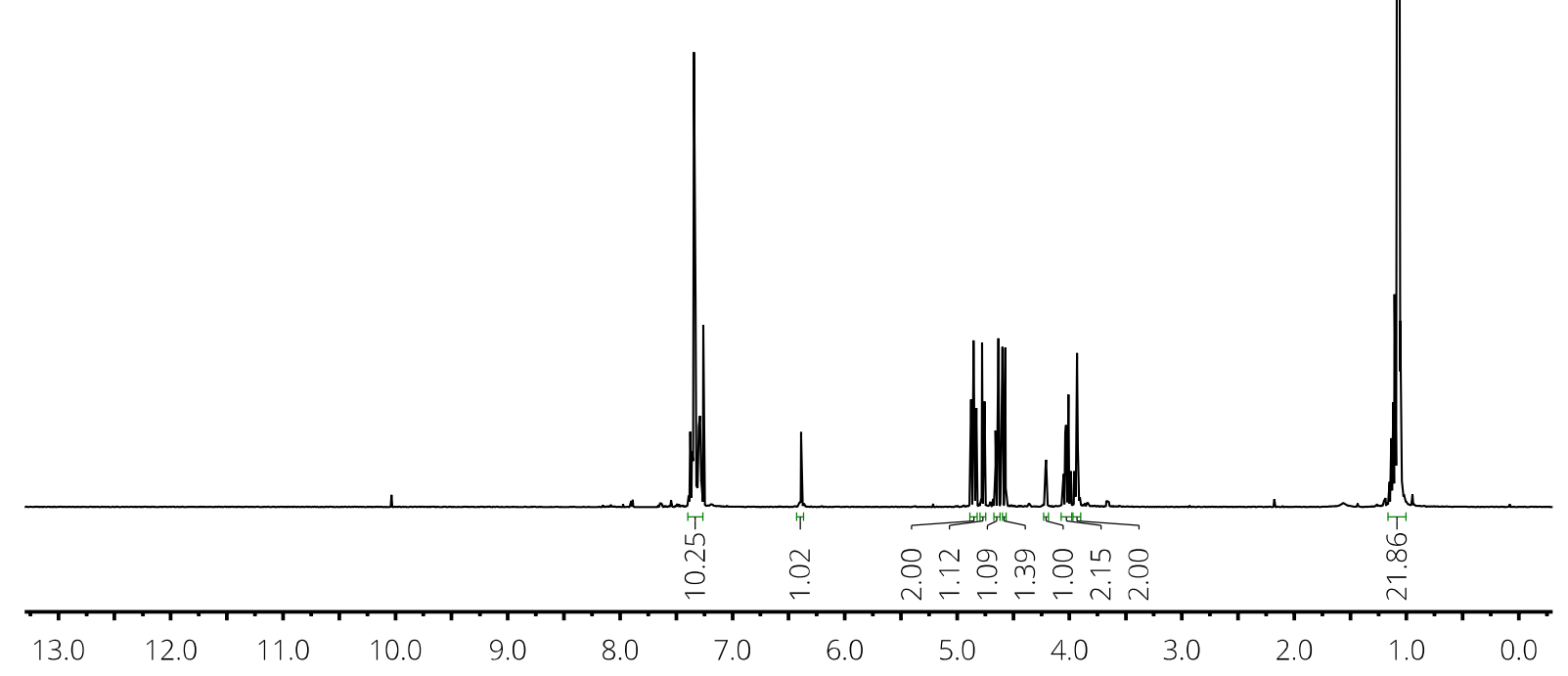




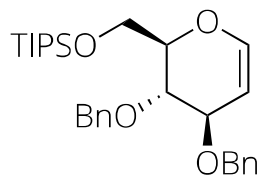

$\mathrm{CDCl}_{3}, 126 \mathrm{MHz}$

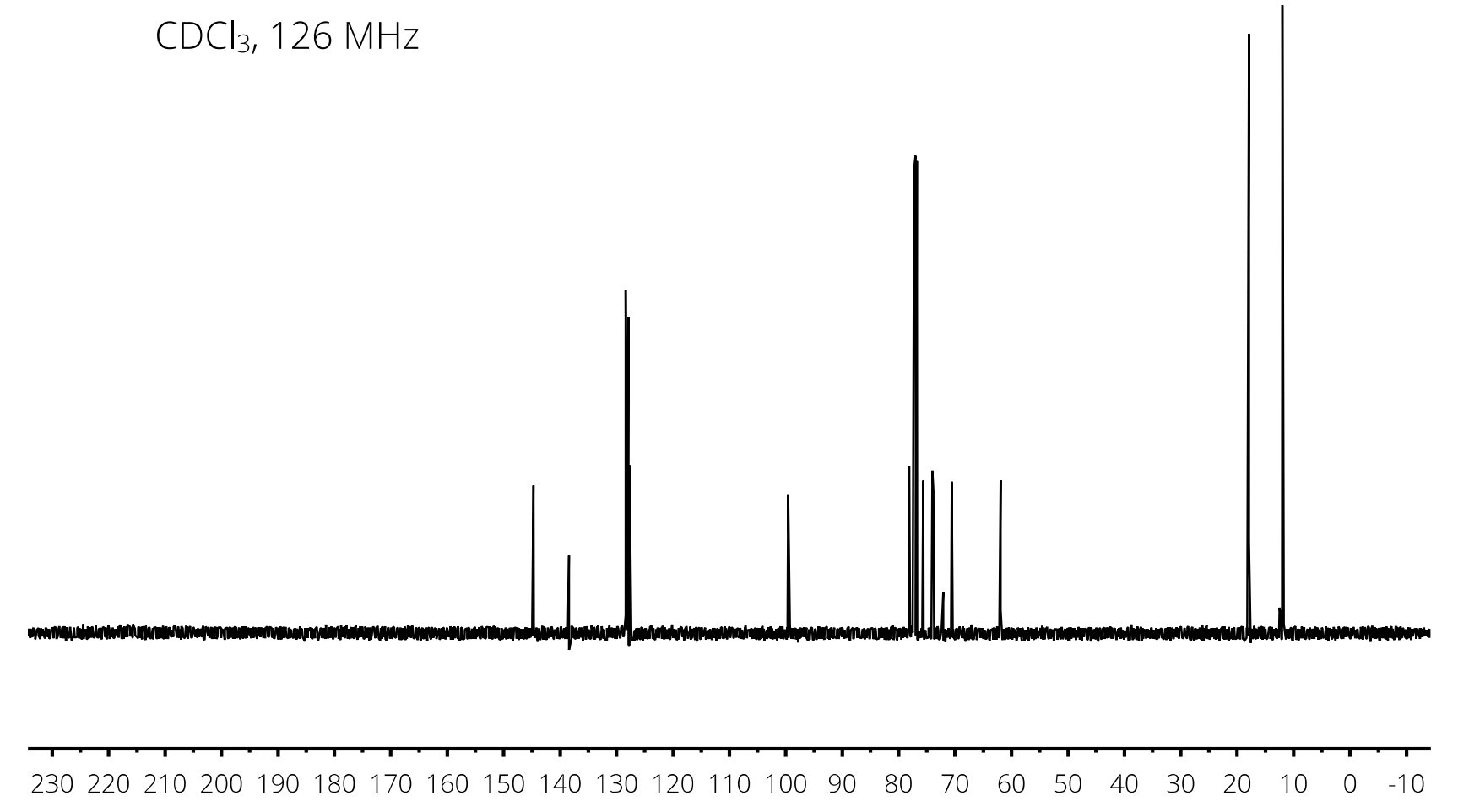




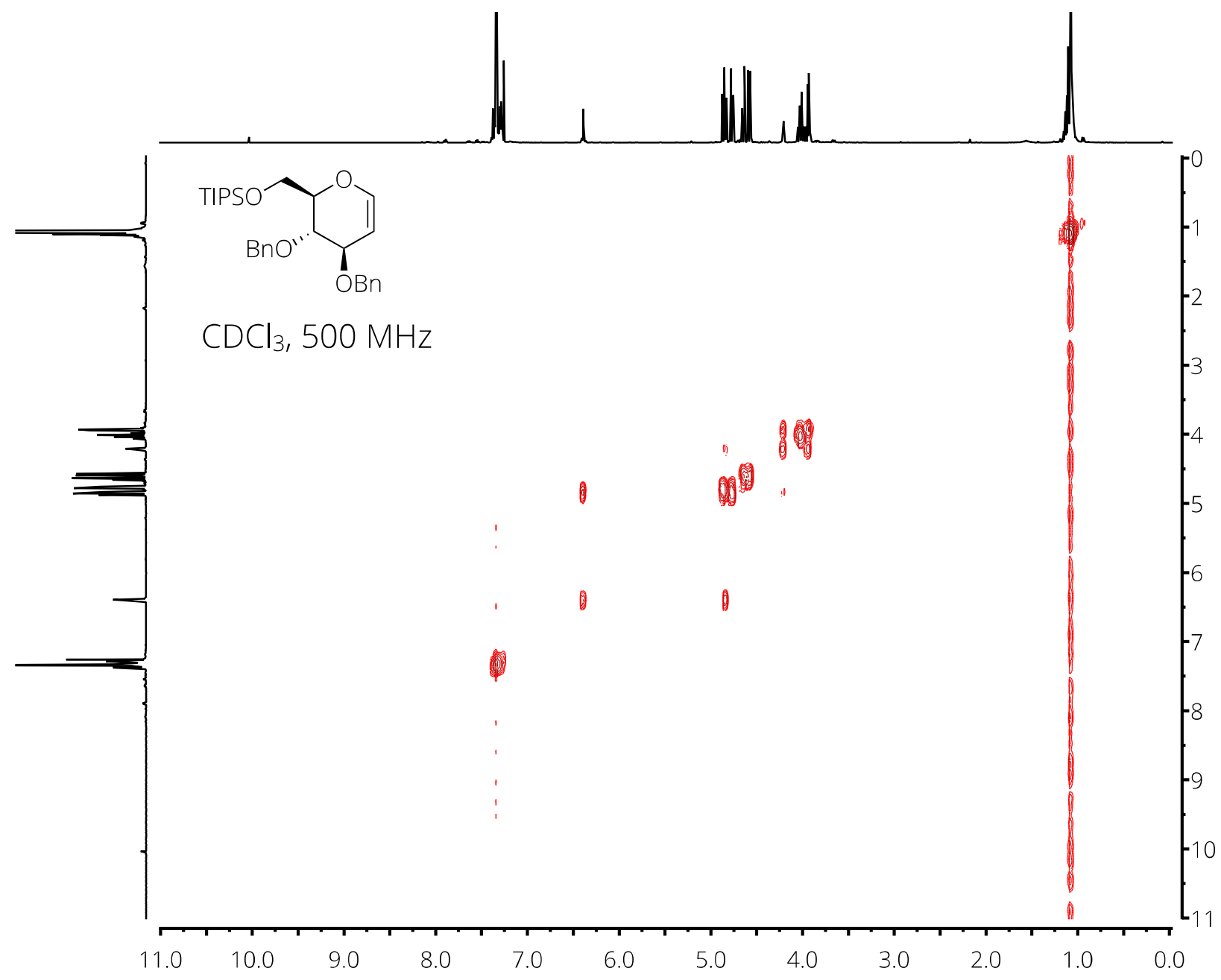




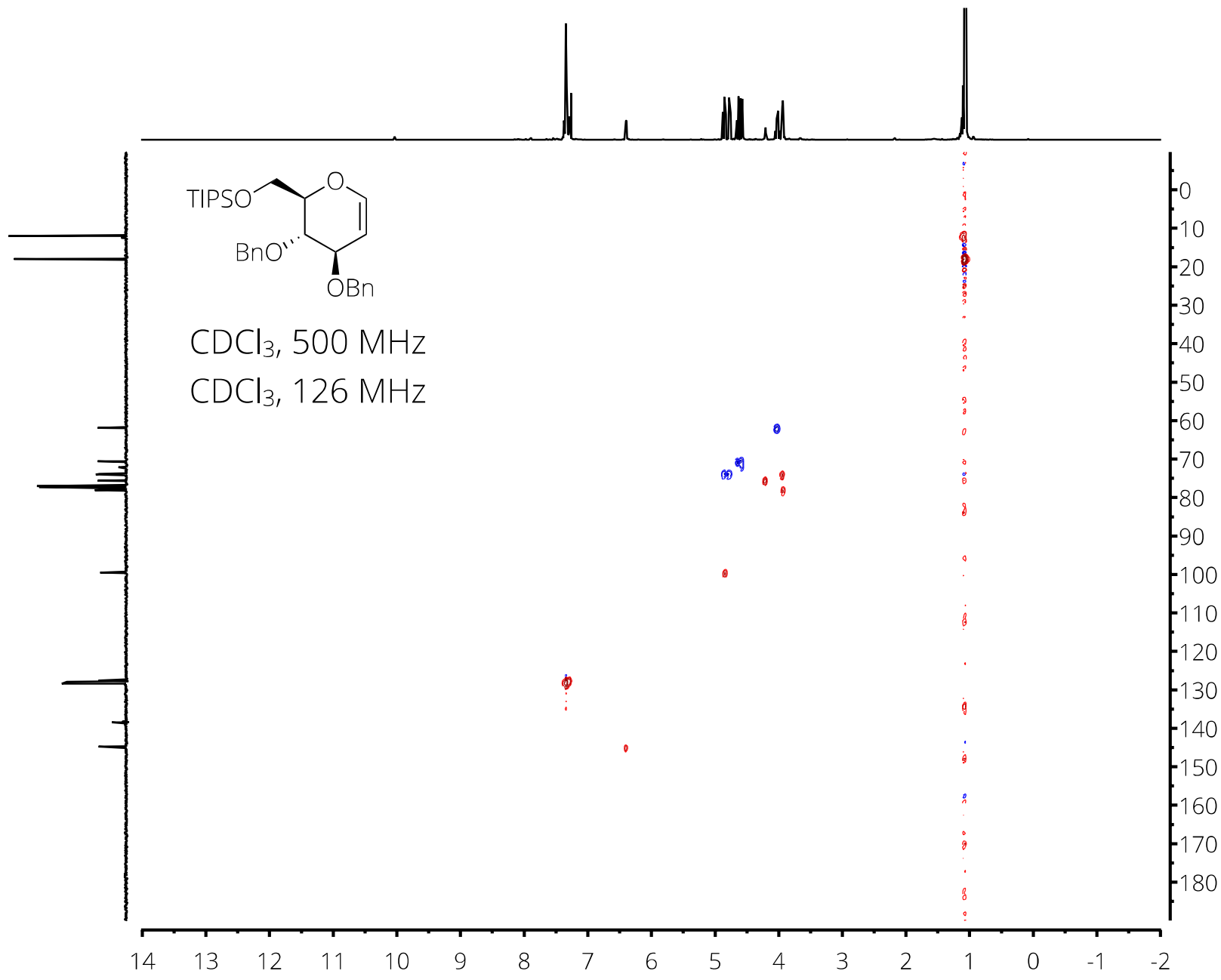


3,4-di-O-benzyl-6-O-acetyl-D-glucal (13) - ${ }^{1} \mathrm{H}-\mathrm{NMR}$

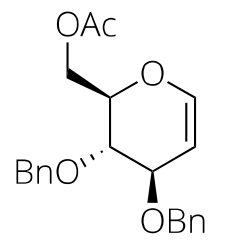

$\mathrm{CDCl}_{3}, 500 \mathrm{MHz}$

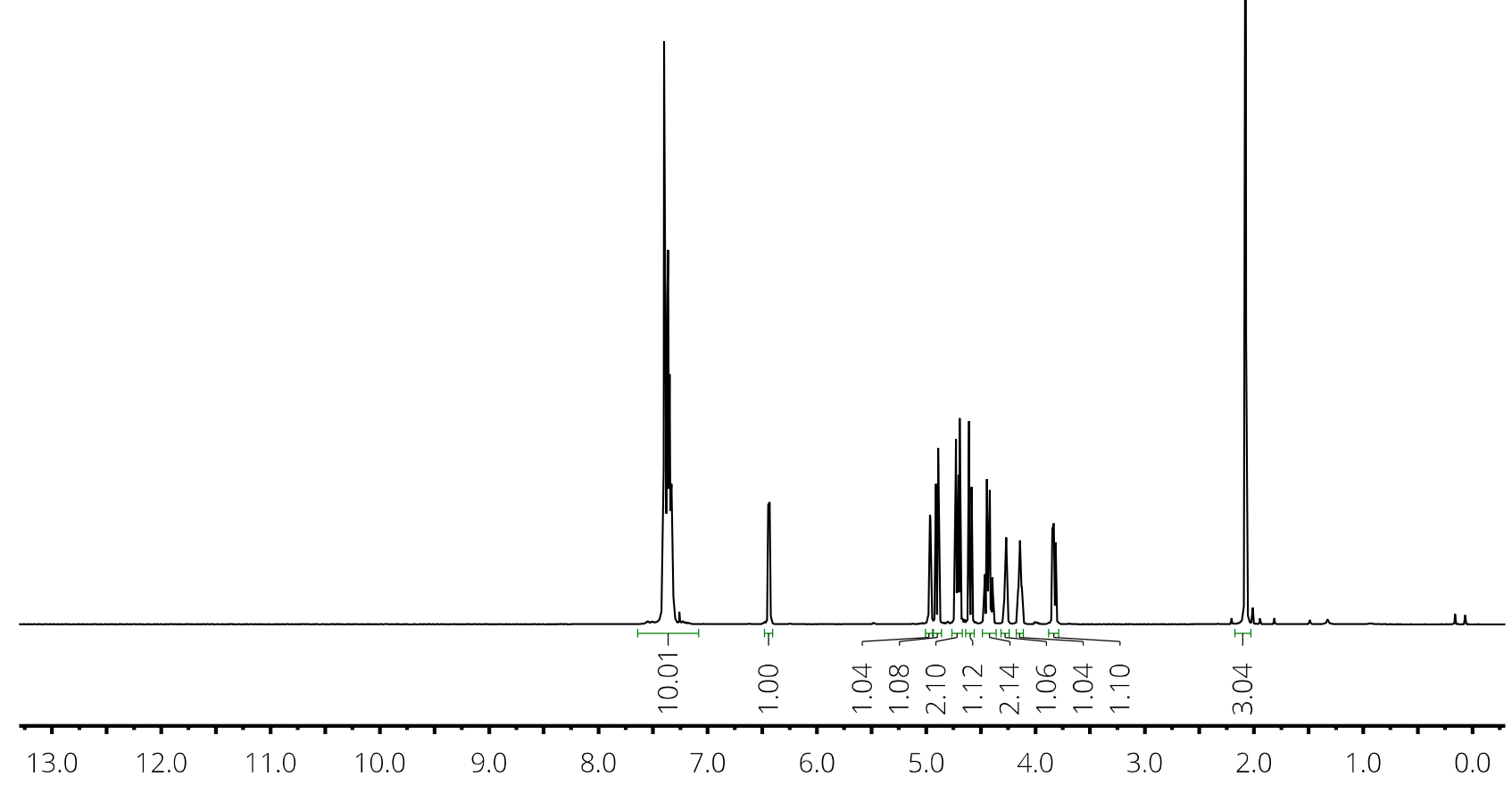


3,4-di- $O$-benzyl-6-O-acetyl-D-glucal (13) - ${ }^{13} \mathrm{C}\left\{{ }^{1} \mathrm{H}\right\}$-NMR

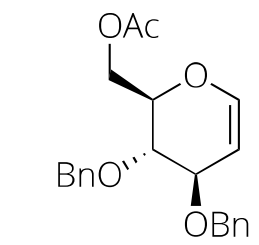

$\mathrm{CDCl}_{3}, 126 \mathrm{MHz}$

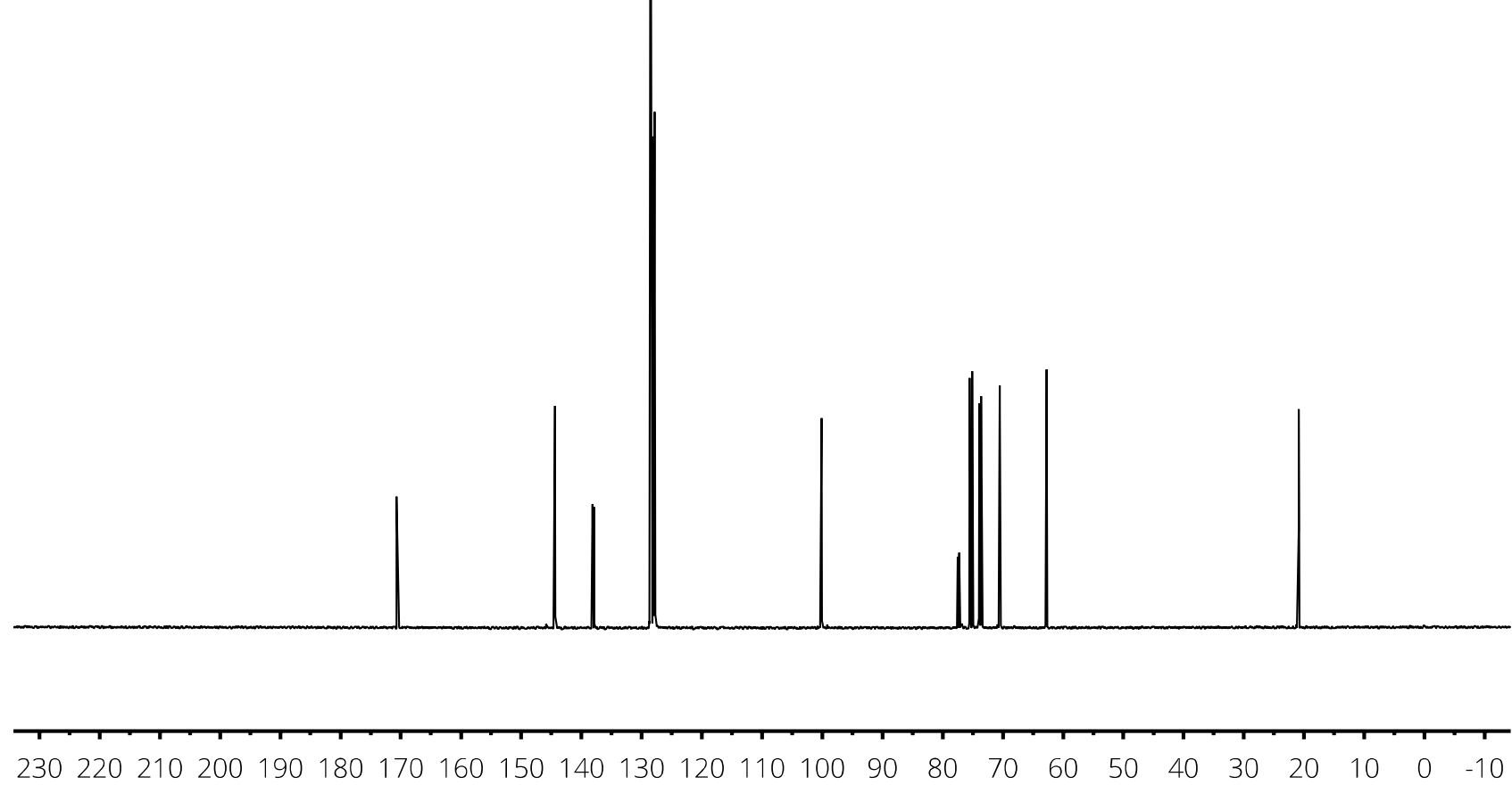


3,4-di-O-benzyl-6-O-acetyl-D-glucal (13) - ${ }^{1} \mathrm{H}^{1} \mathrm{H}-\mathrm{COSY}$

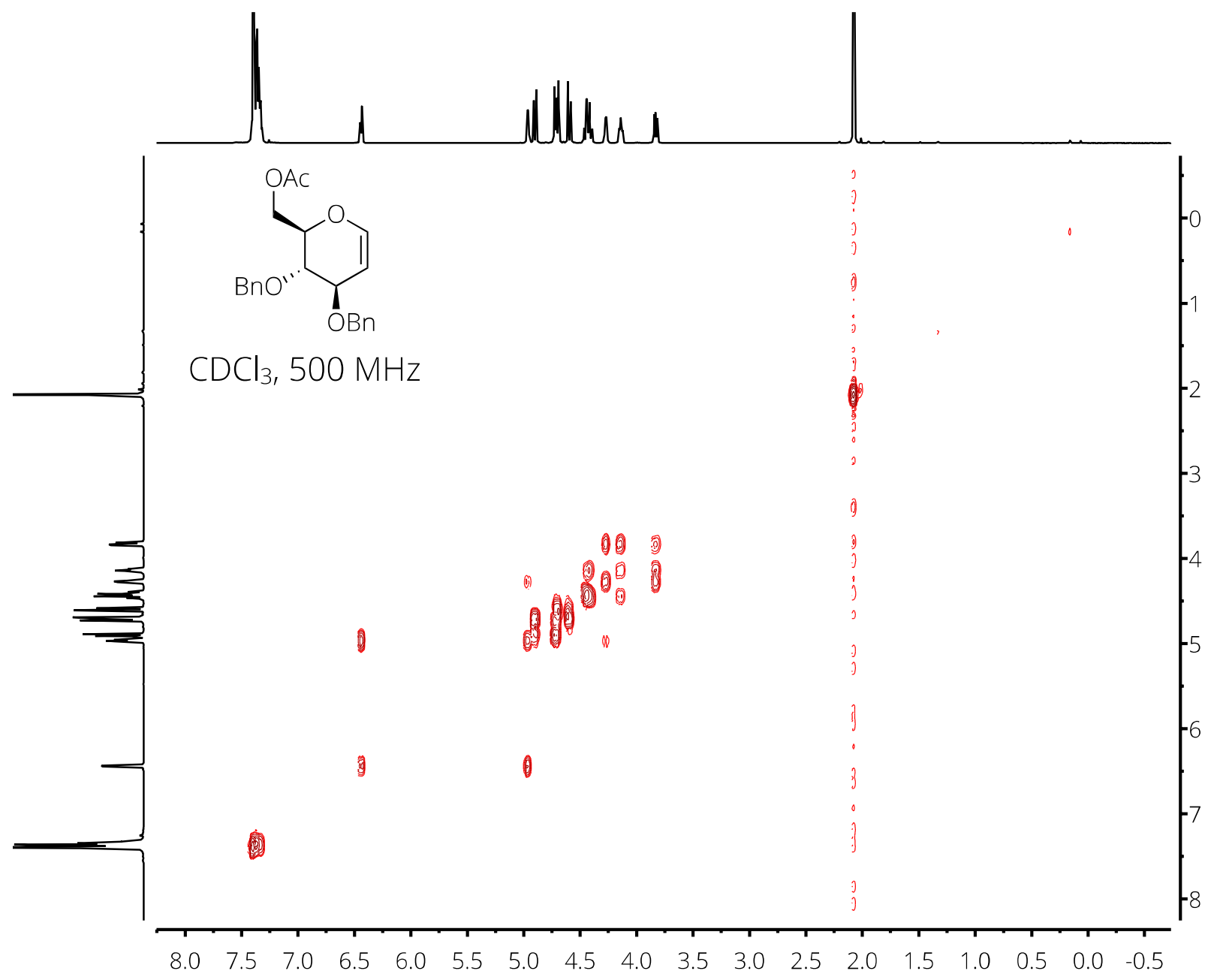

S

75 


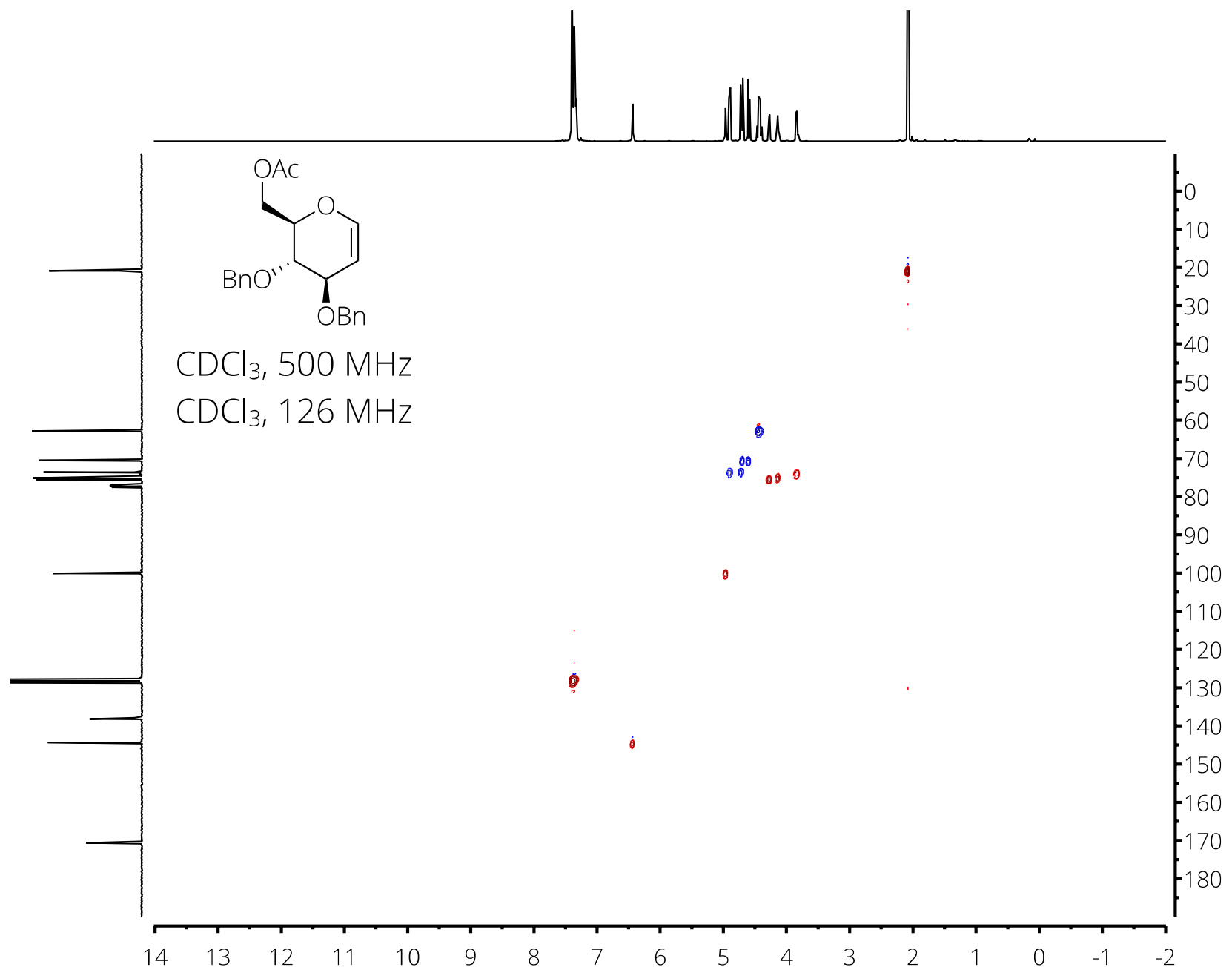




\section{3,4-di-O-benzyl-6-O-methyl-D-glucal (14) - ${ }^{1} \mathrm{H}-\mathrm{NMR}$}

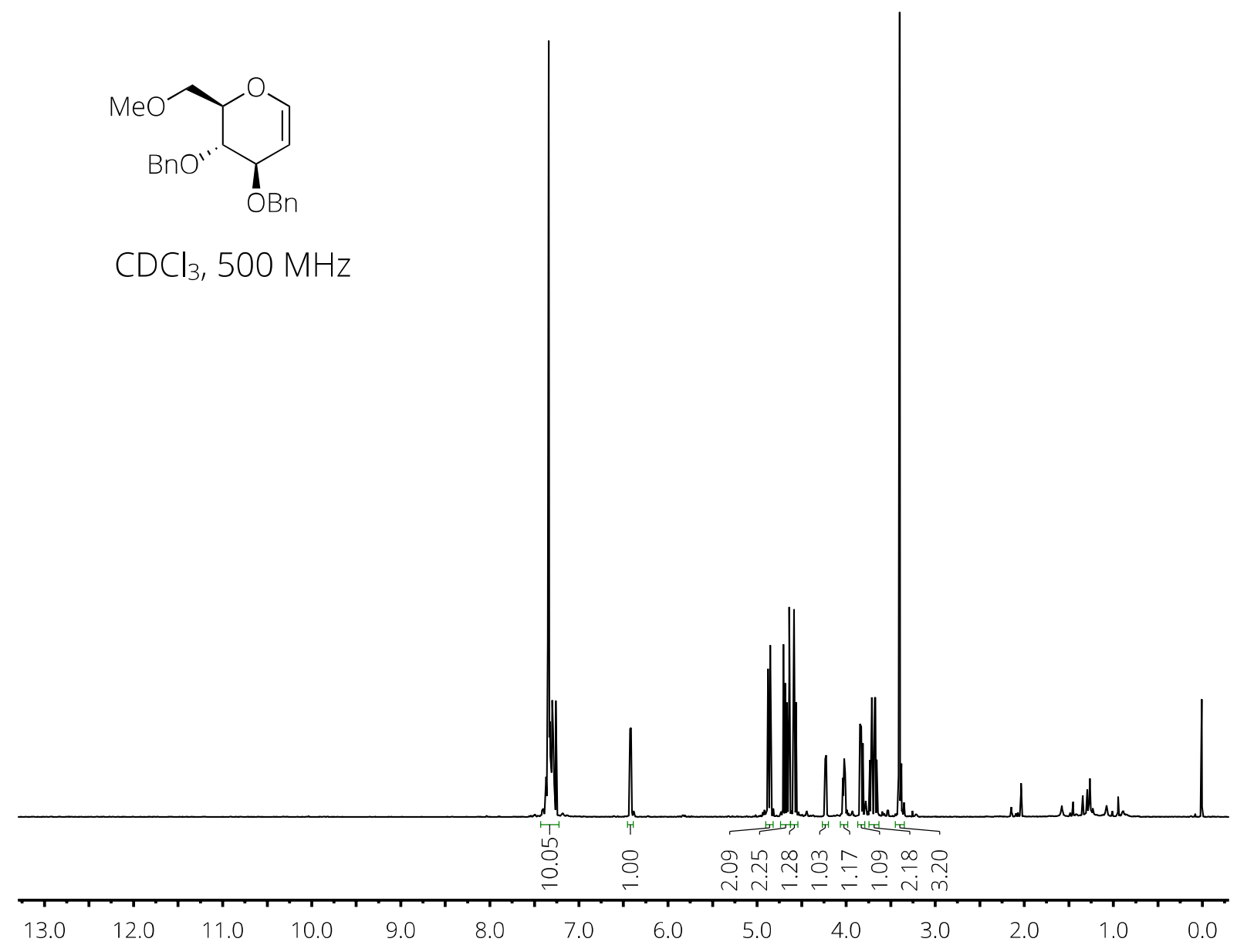




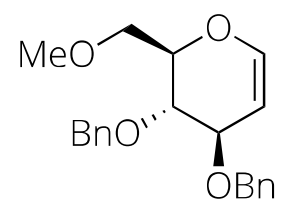

$\mathrm{CDCl}_{3}, 126 \mathrm{MHz}$

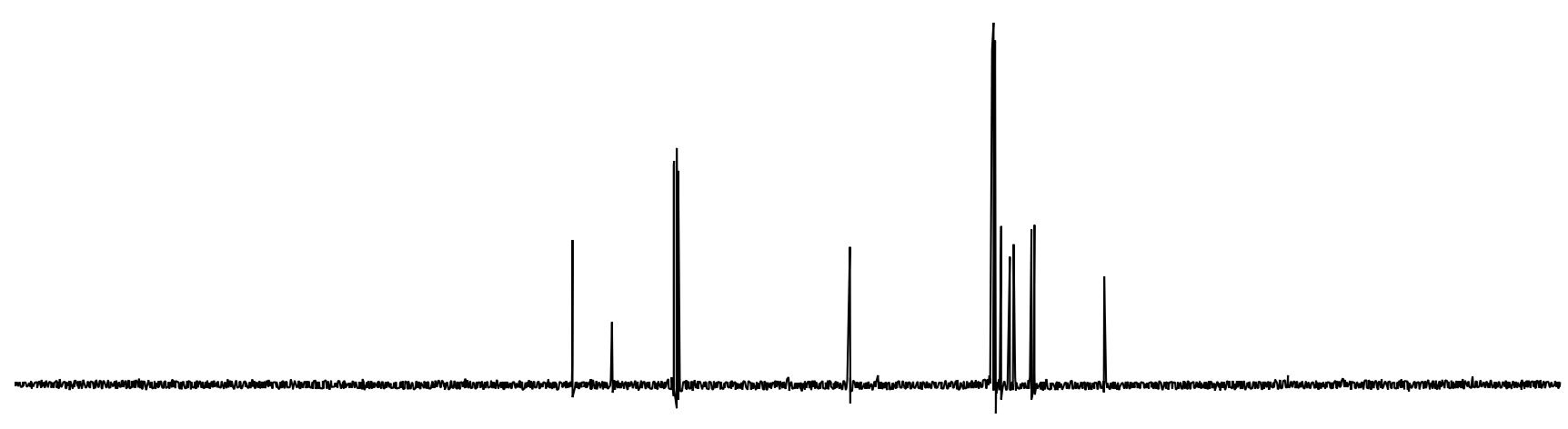

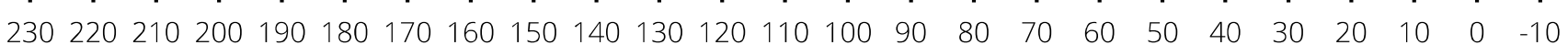




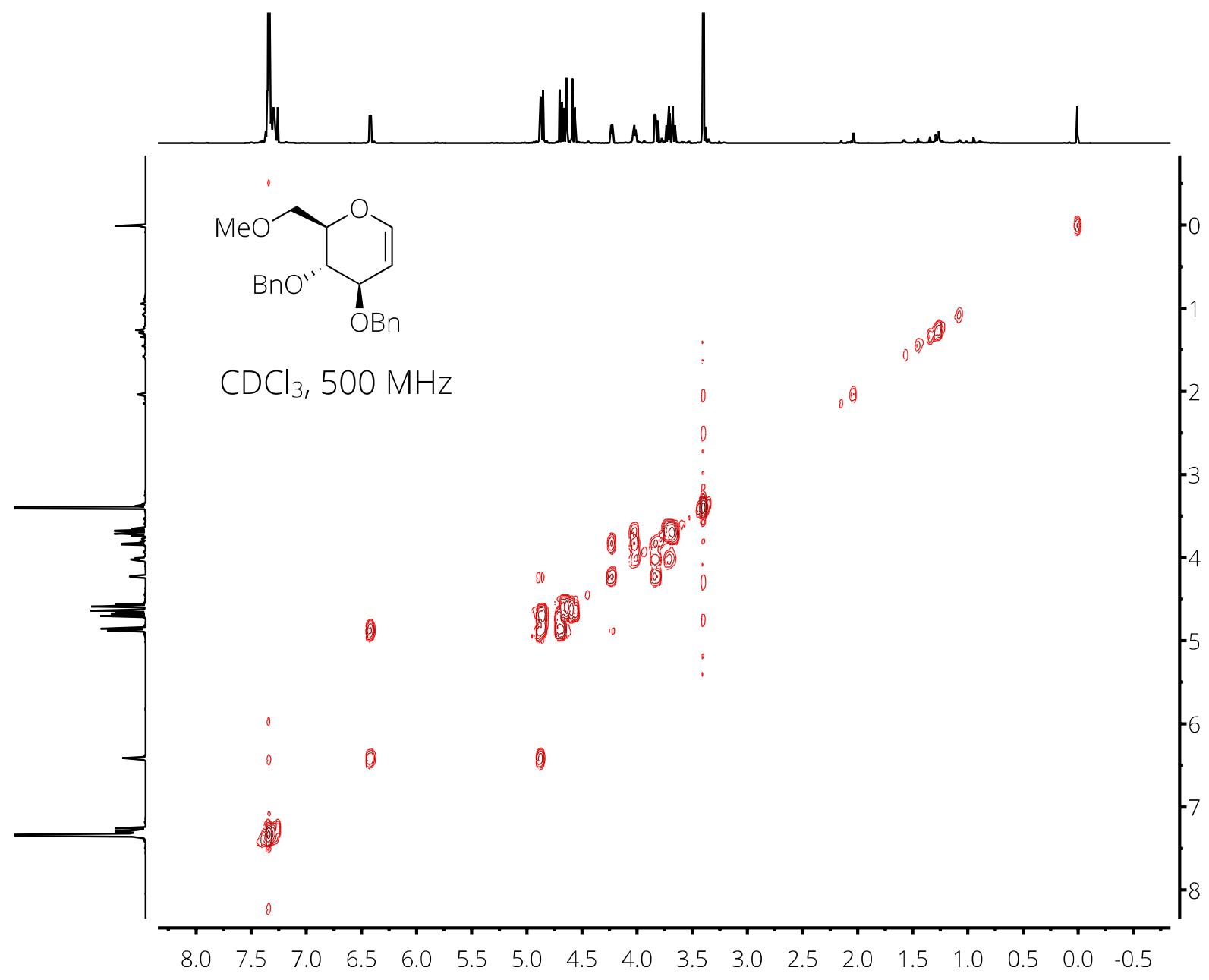




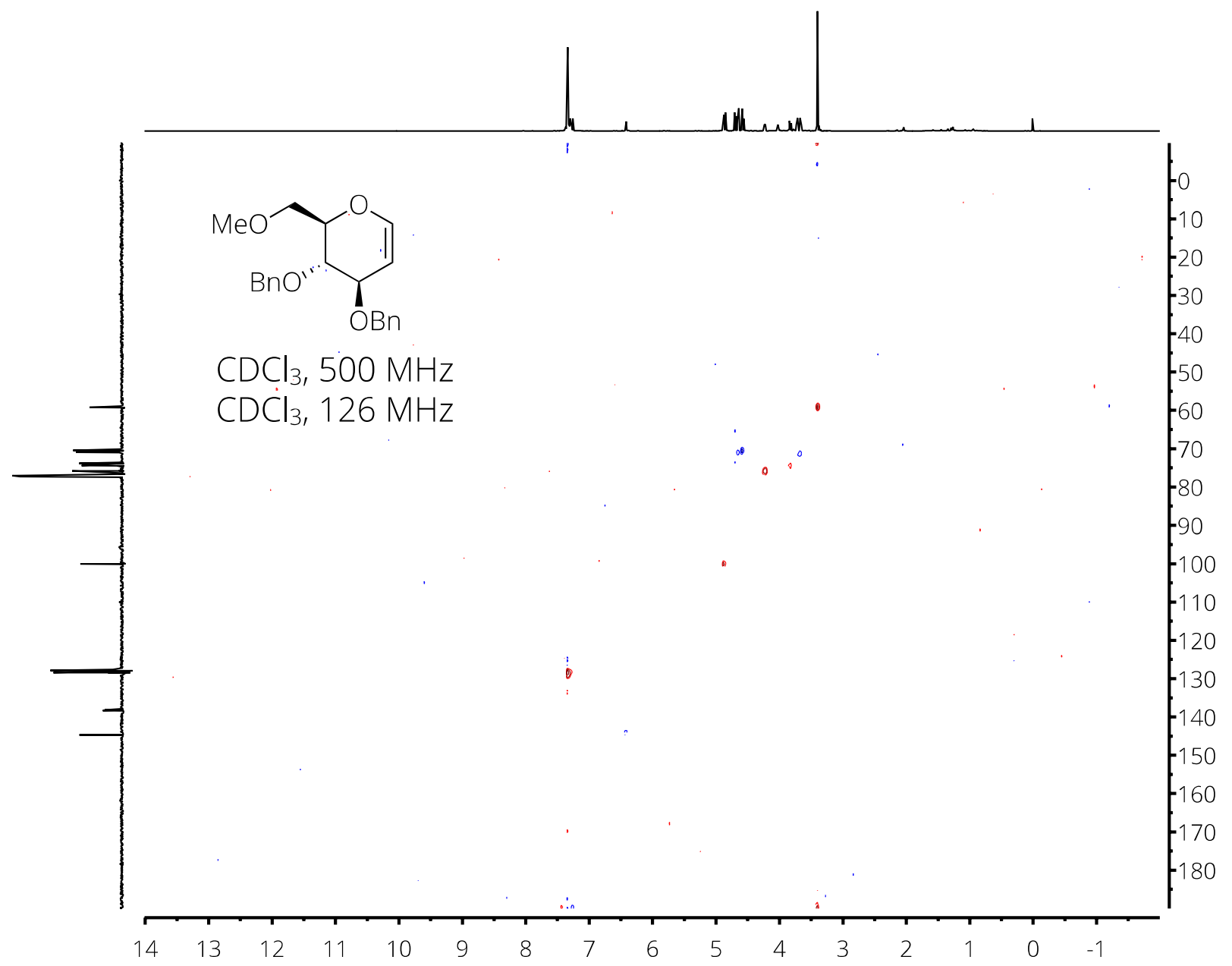


3,4-di-O-benzyl-6-O-(2,2,2-trichloroethylcarbonate)-D-glucal (15) - ${ }^{1} \mathrm{H}-\mathrm{NMR}$

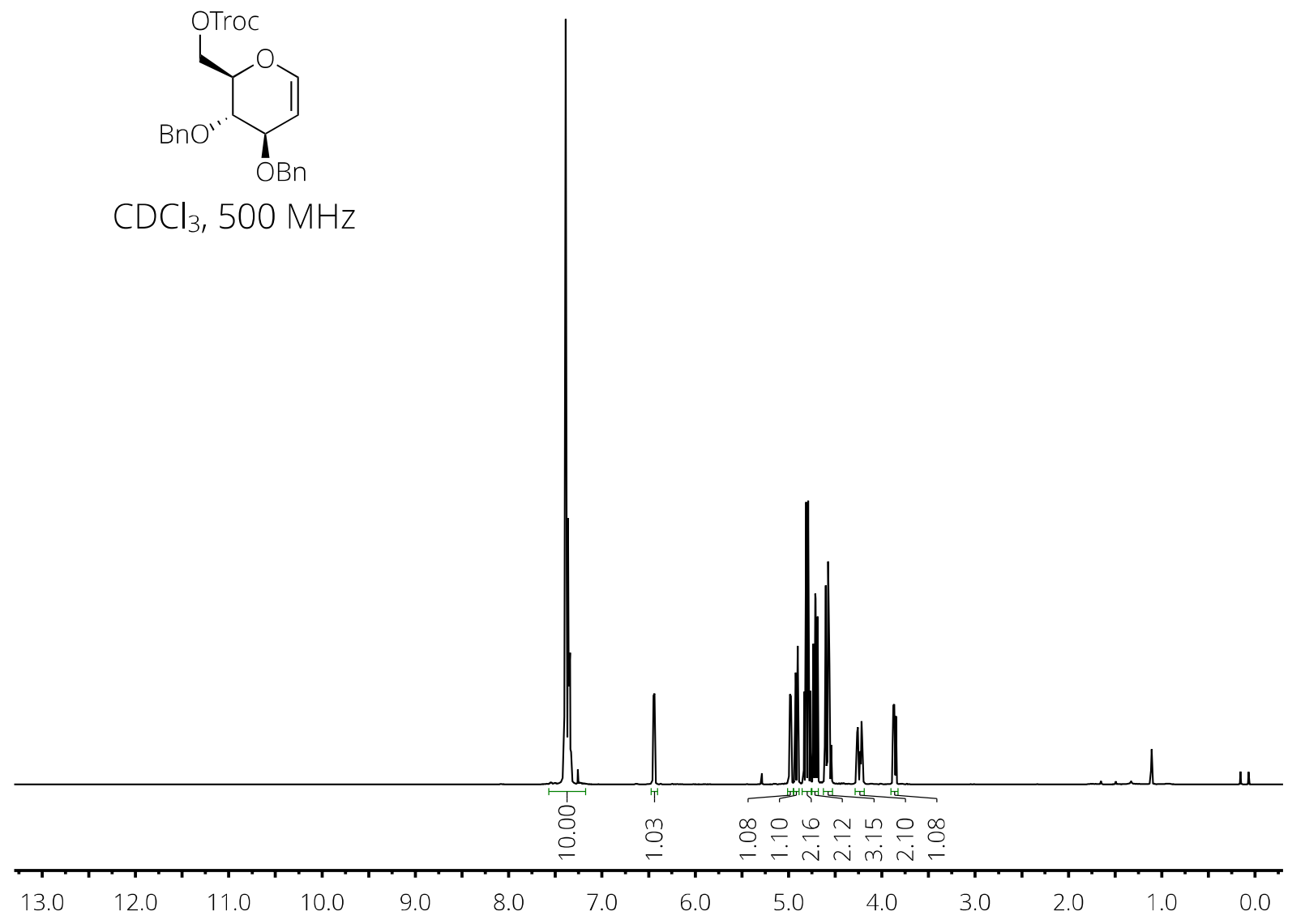


3,4-di- $O$-benzyl-6-O-(2,2,2-trichloroethylcarbonate)-D-glucal (15) - ${ }^{13} \mathrm{C}\left\{{ }^{1} \mathrm{H}\right\}$-NMR

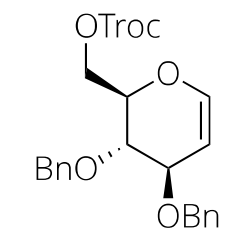

$\mathrm{CDCl}_{3}, 126 \mathrm{MHz}$

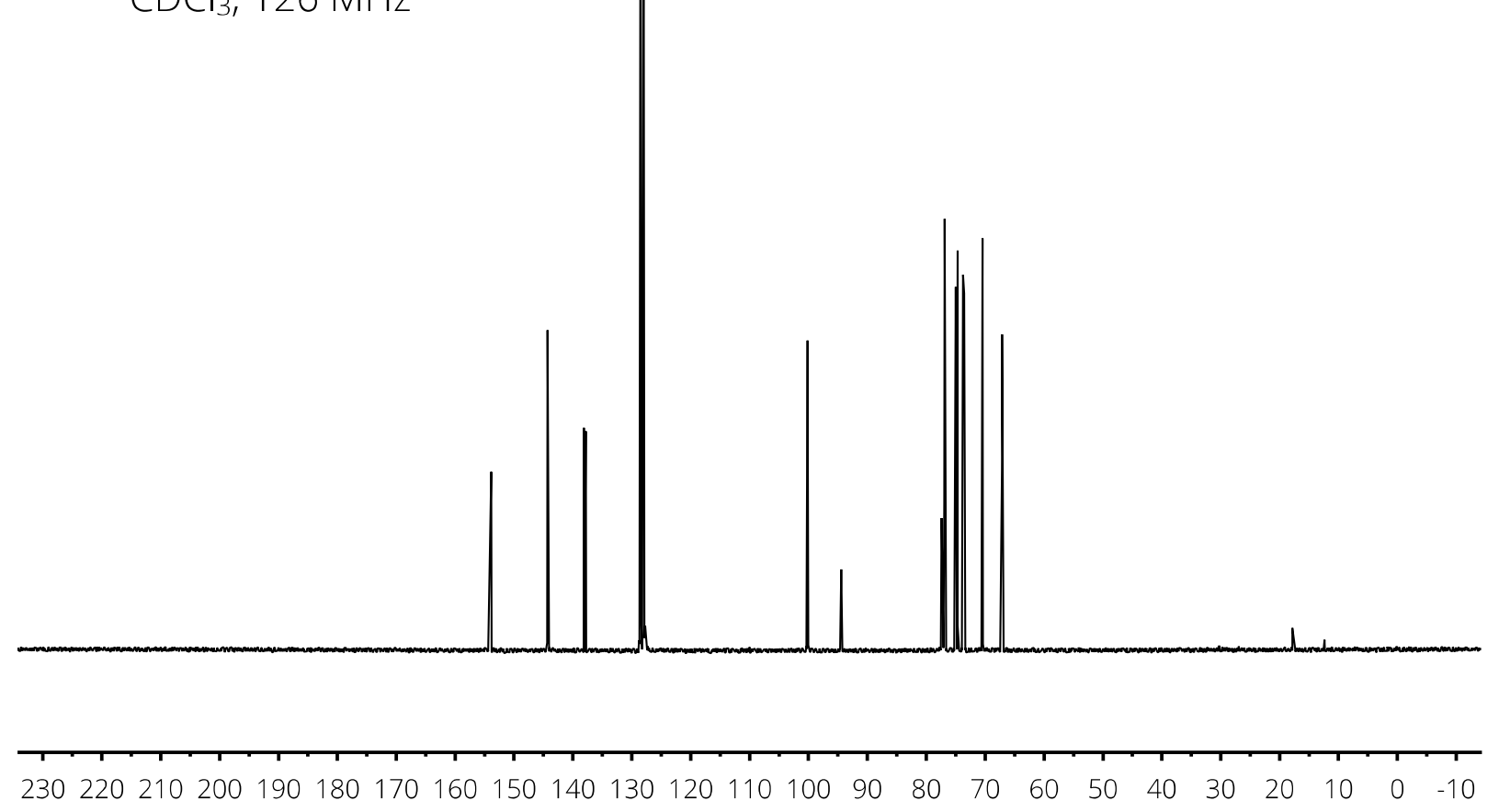


3,4-di-O-benzyl-6-O-(2,2,2-trichloroethylcarbonate)-D-glucal (15) - ${ }^{1} \mathrm{H}^{1} \mathrm{H}-\mathrm{COSY}$

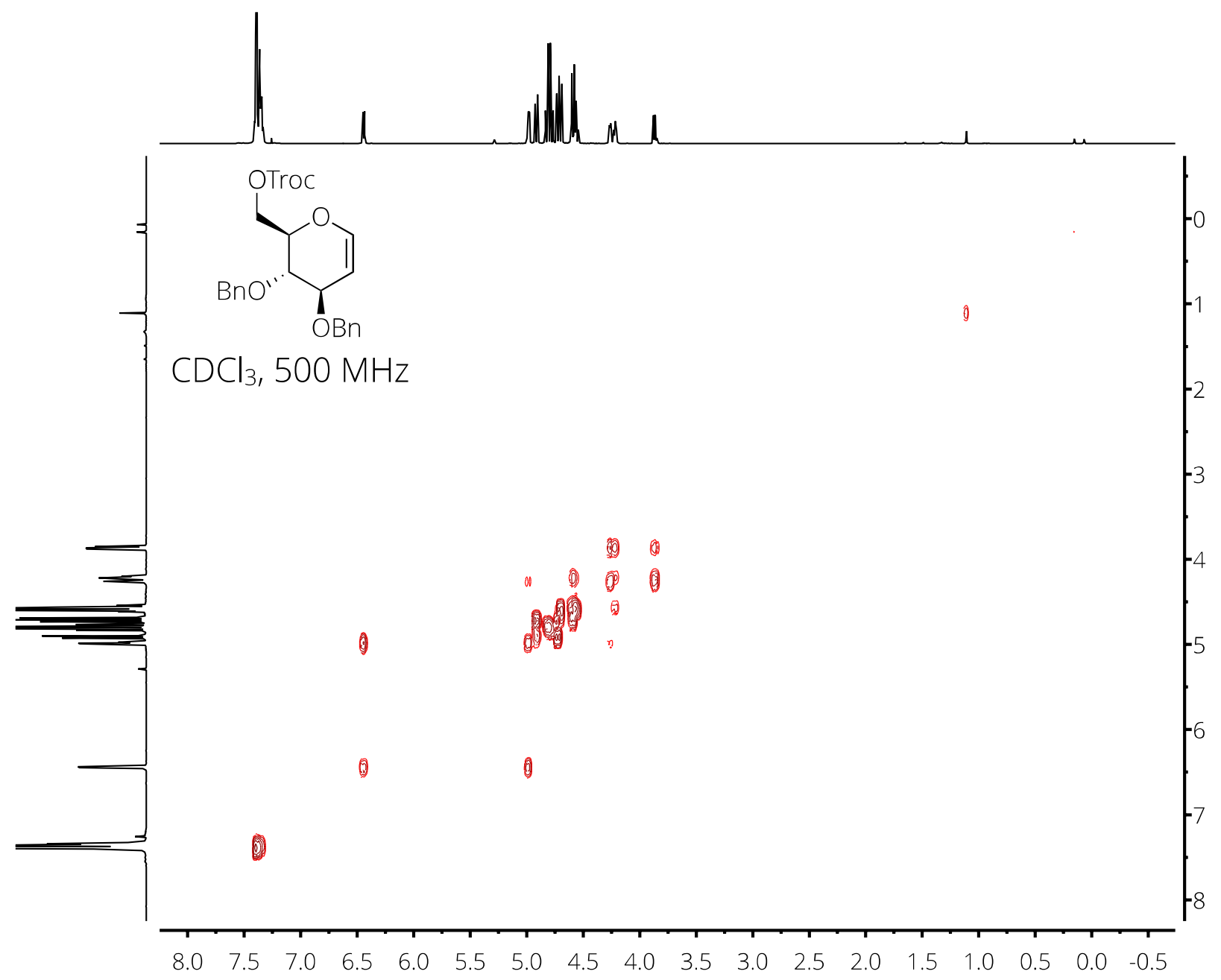




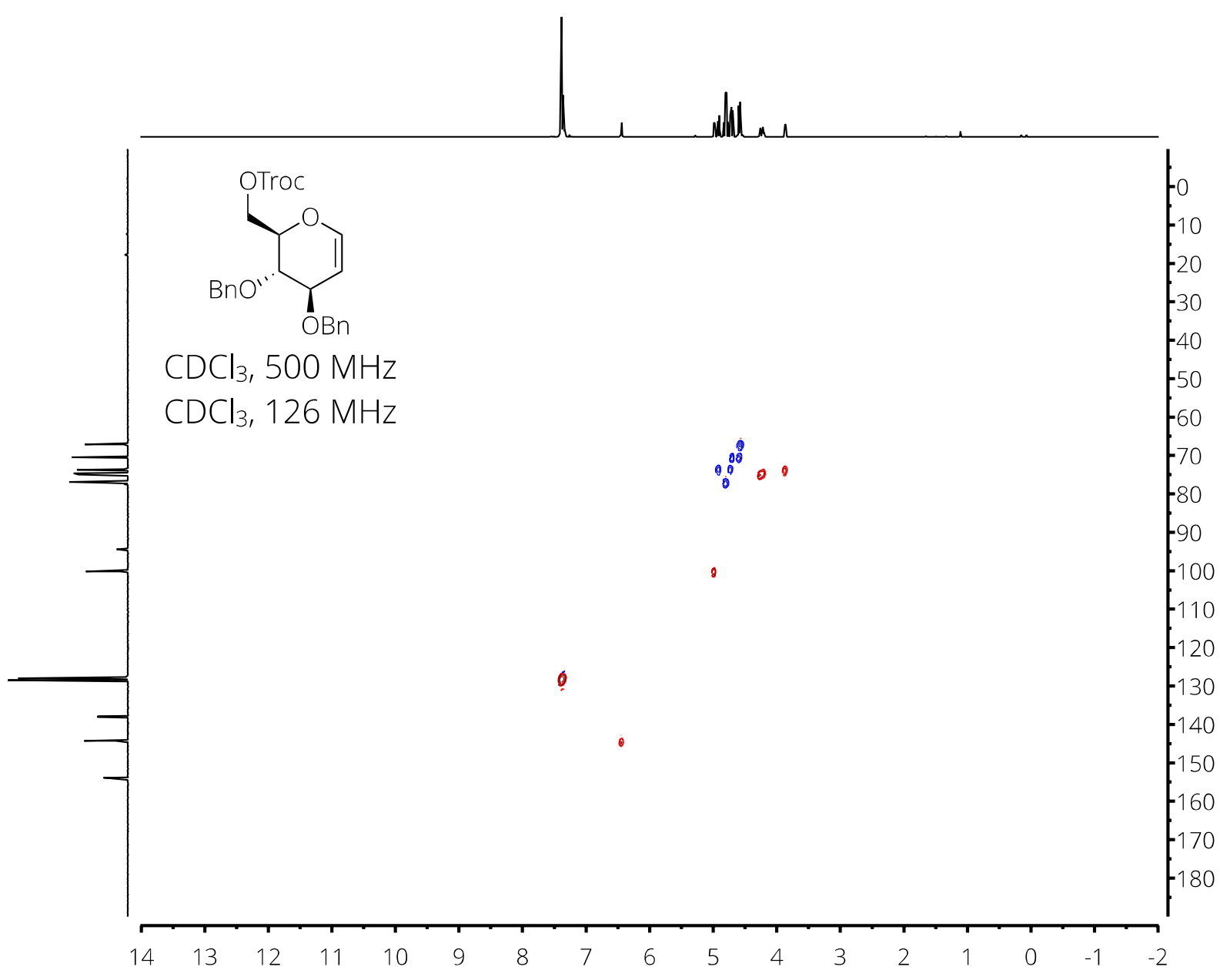


3,4-di-O-benzyl-6-O-trityl-D-glucal (16) - ${ }^{1} \mathrm{H}-\mathrm{NMR}$

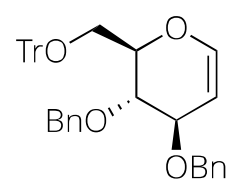

$\mathrm{CDCl}_{3}, 500 \mathrm{MHz}$

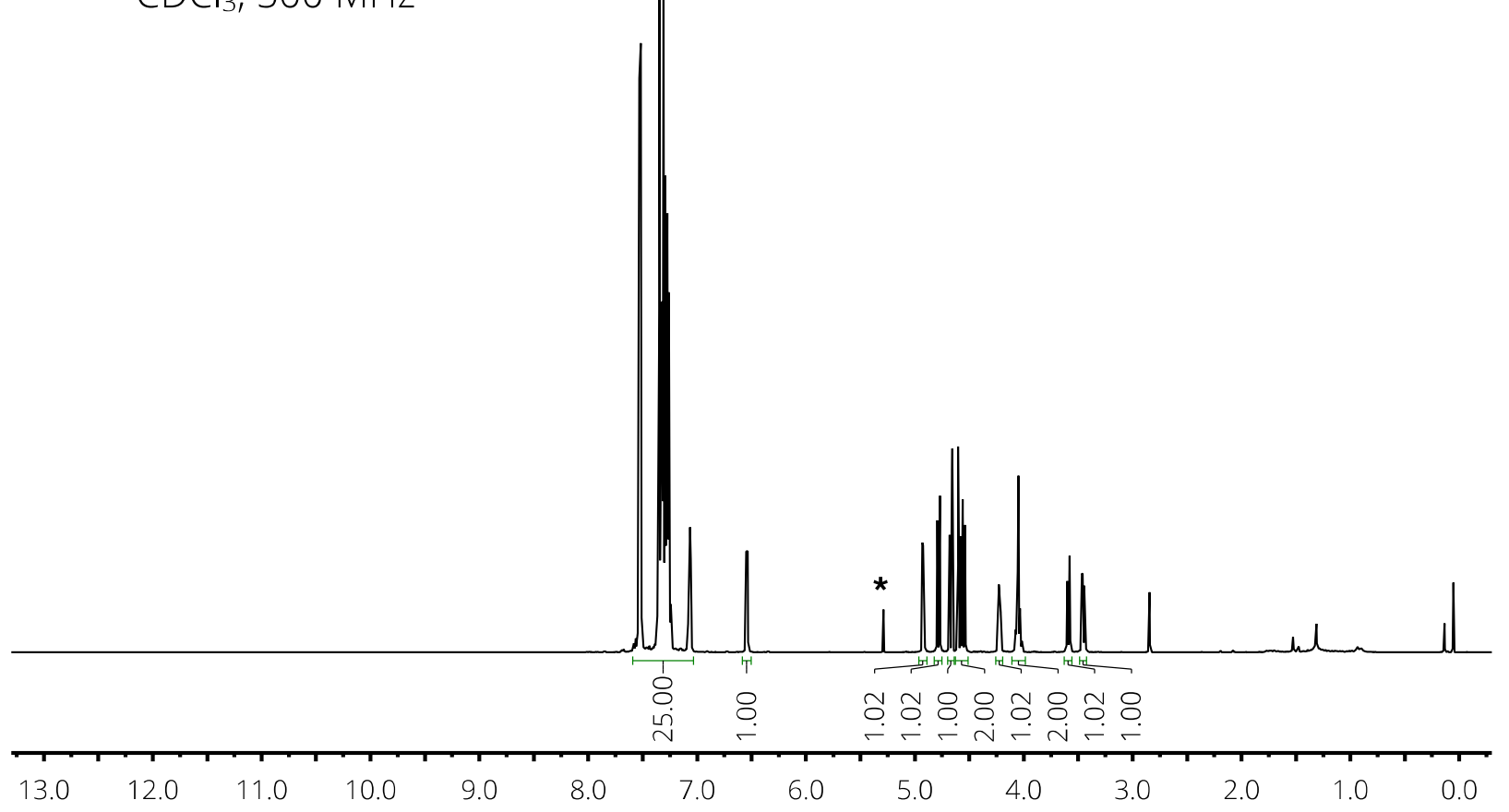

$*=$ solvent $(\mathrm{DCM})$ 


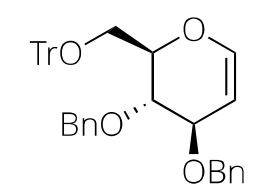

$\mathrm{CDCl}_{3}, 126 \mathrm{MHz}$

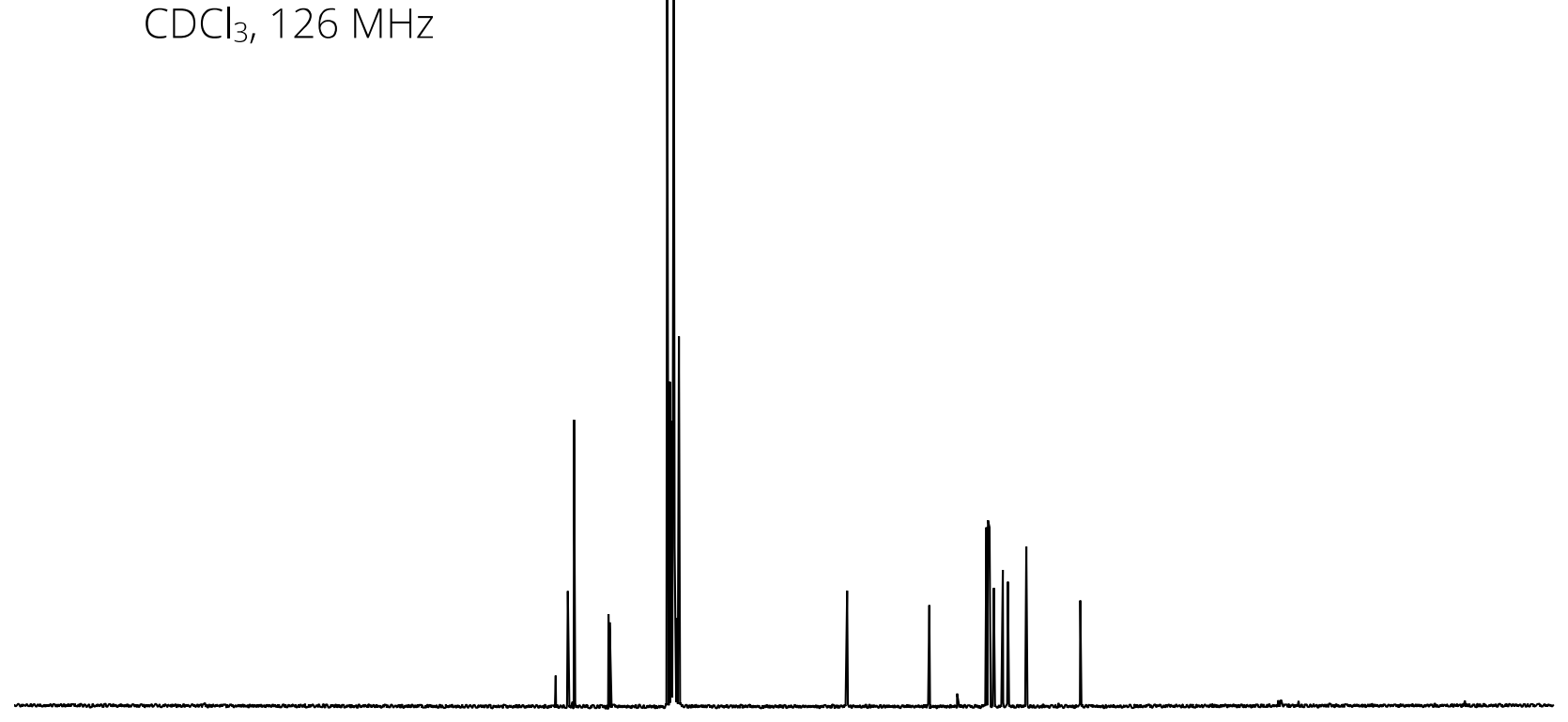

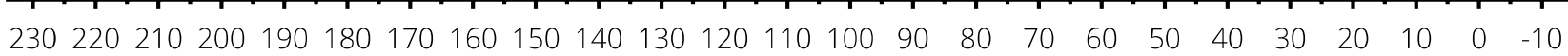


3,4-di-O-benzyl-6-O-trityl-D-glucal (16) - ${ }^{1} \mathrm{H}^{1} \mathrm{H}-\mathrm{COSY}$

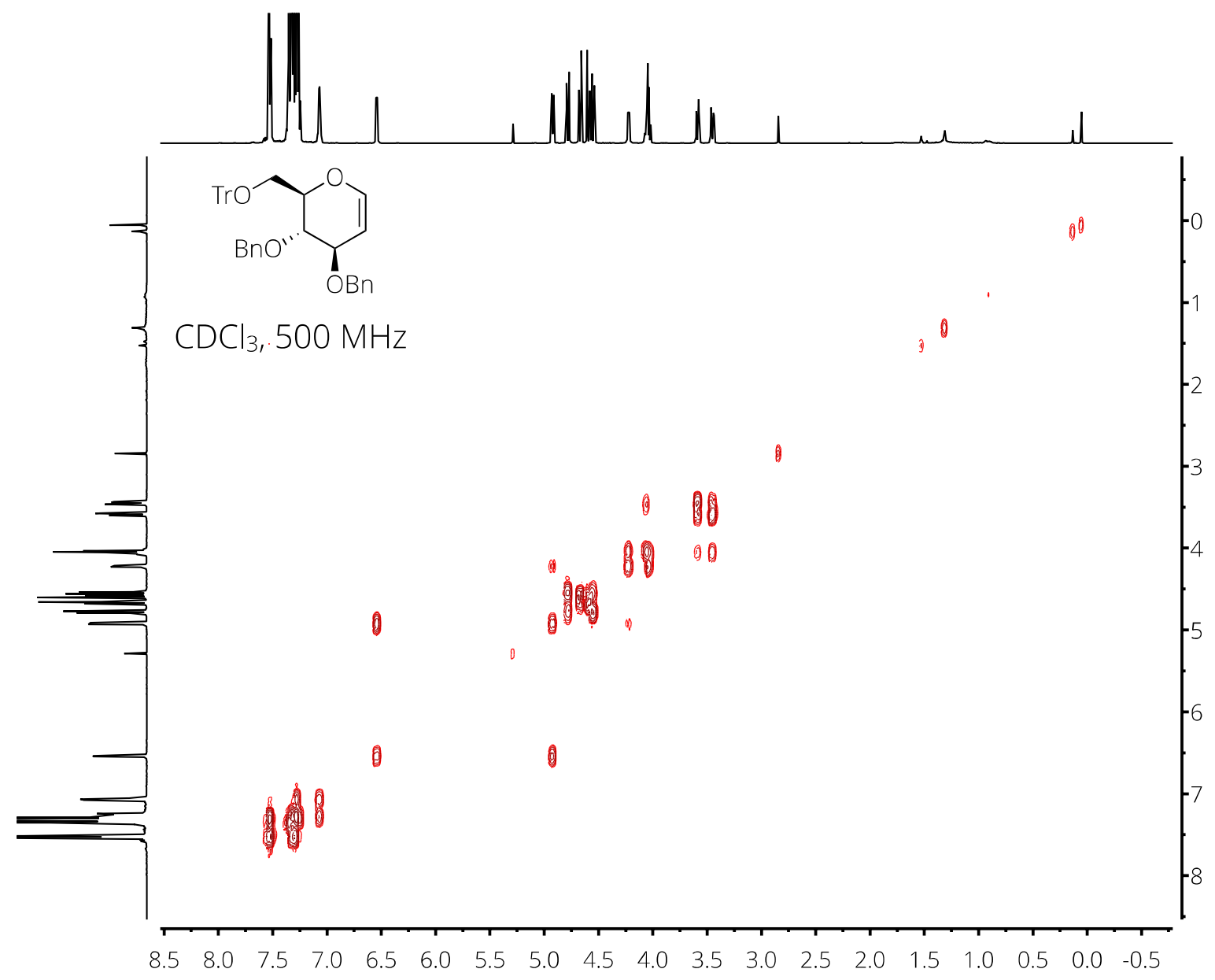




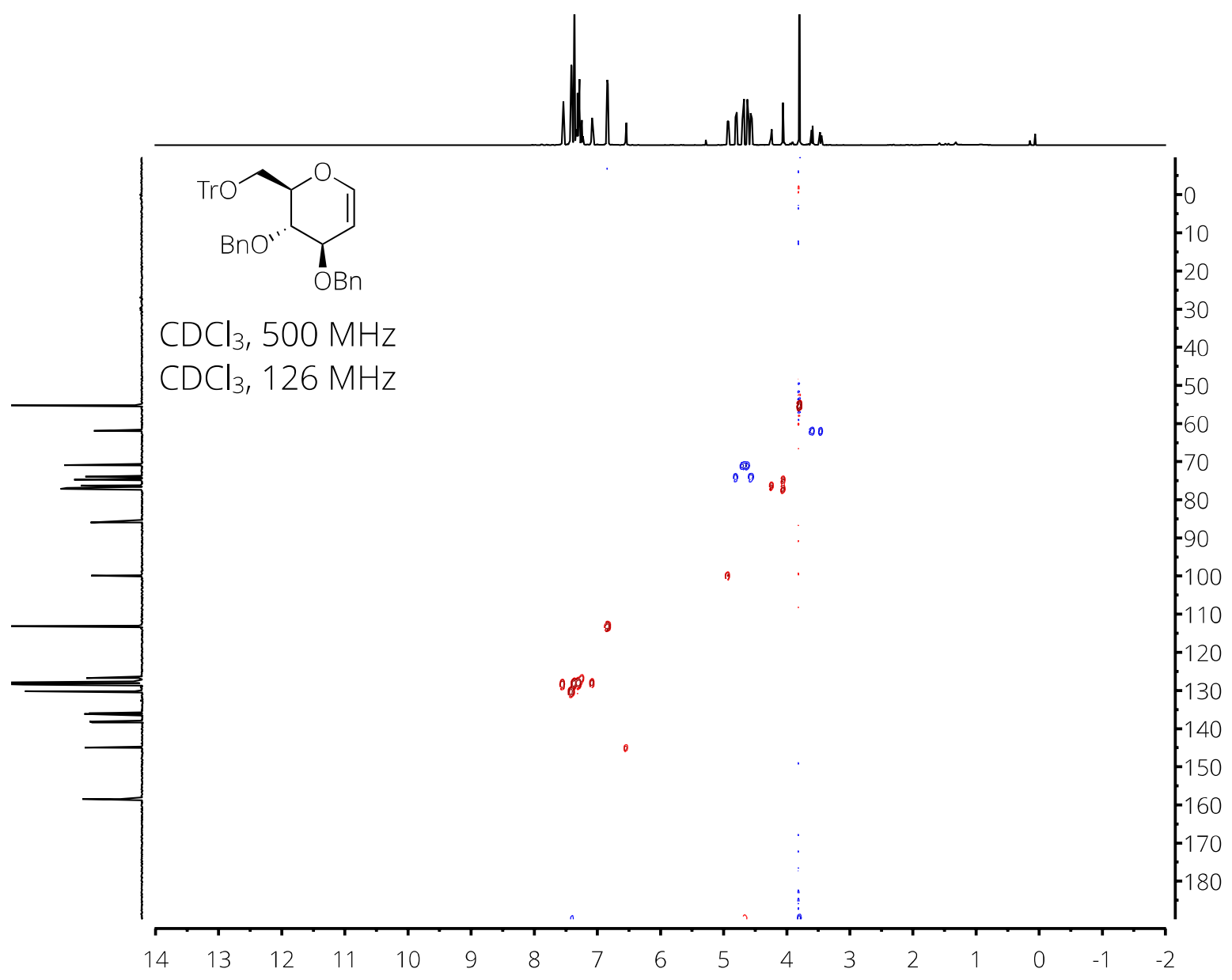


3,4-di-O-benzyl-6-O-(4,4-dimethoxytrityl)-D-glucal (17) - ${ }^{1} \mathrm{H}-\mathrm{NMR}$

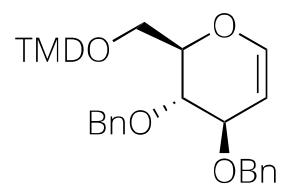

$\mathrm{CDCl}_{3}, 500 \mathrm{MHz}$

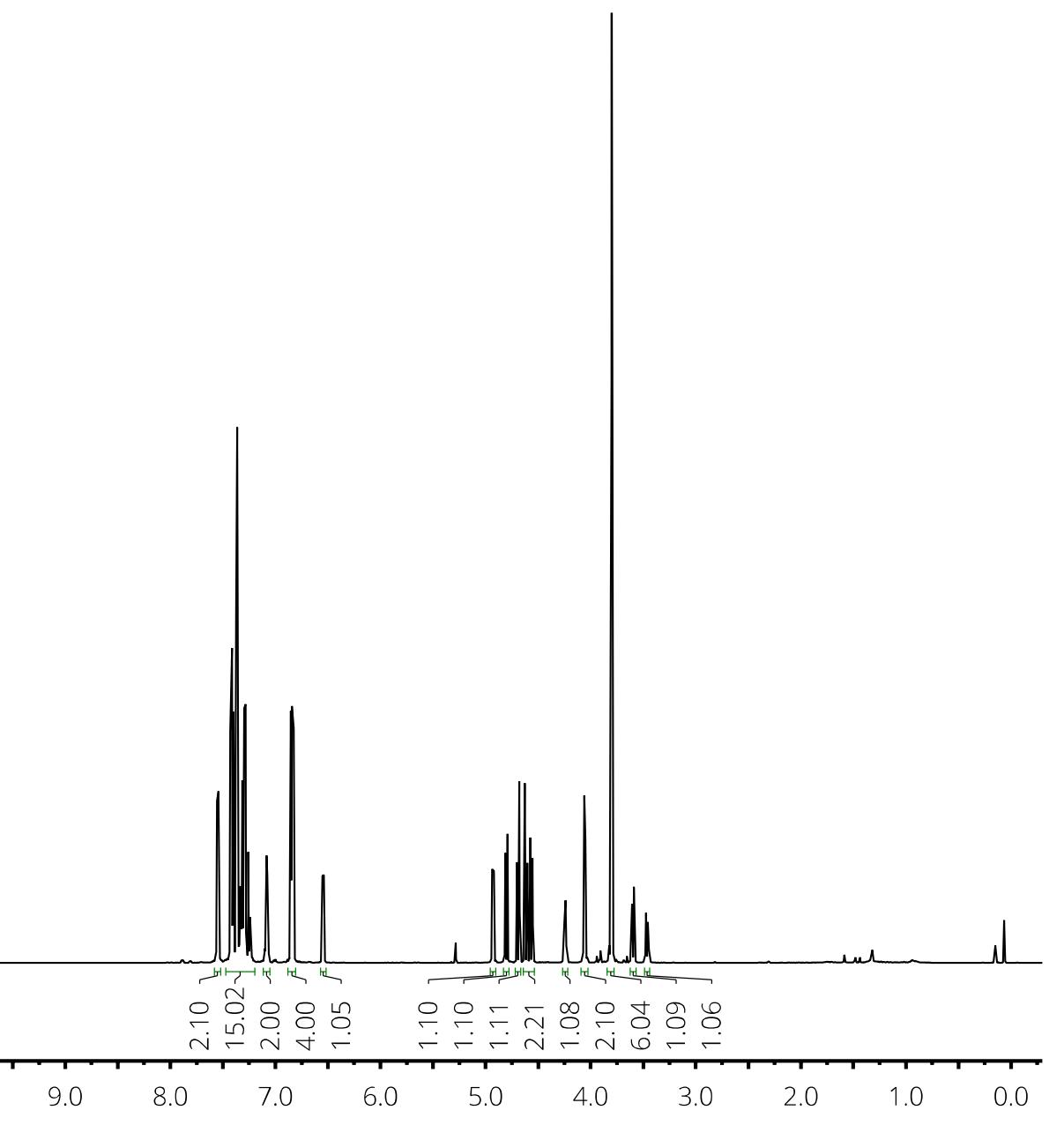




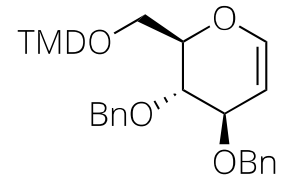

$\mathrm{CDCl}_{3}, 126 \mathrm{MHz}$

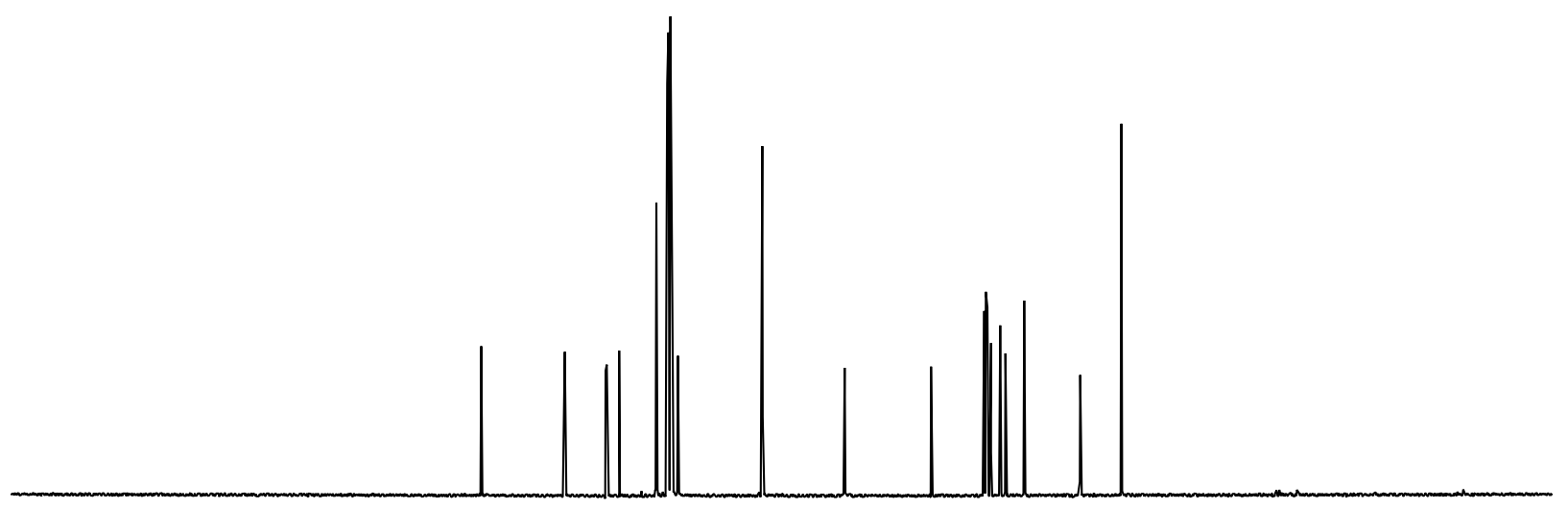

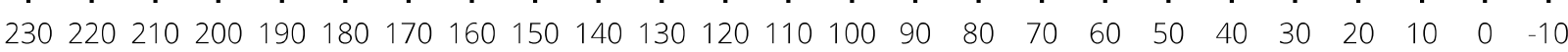




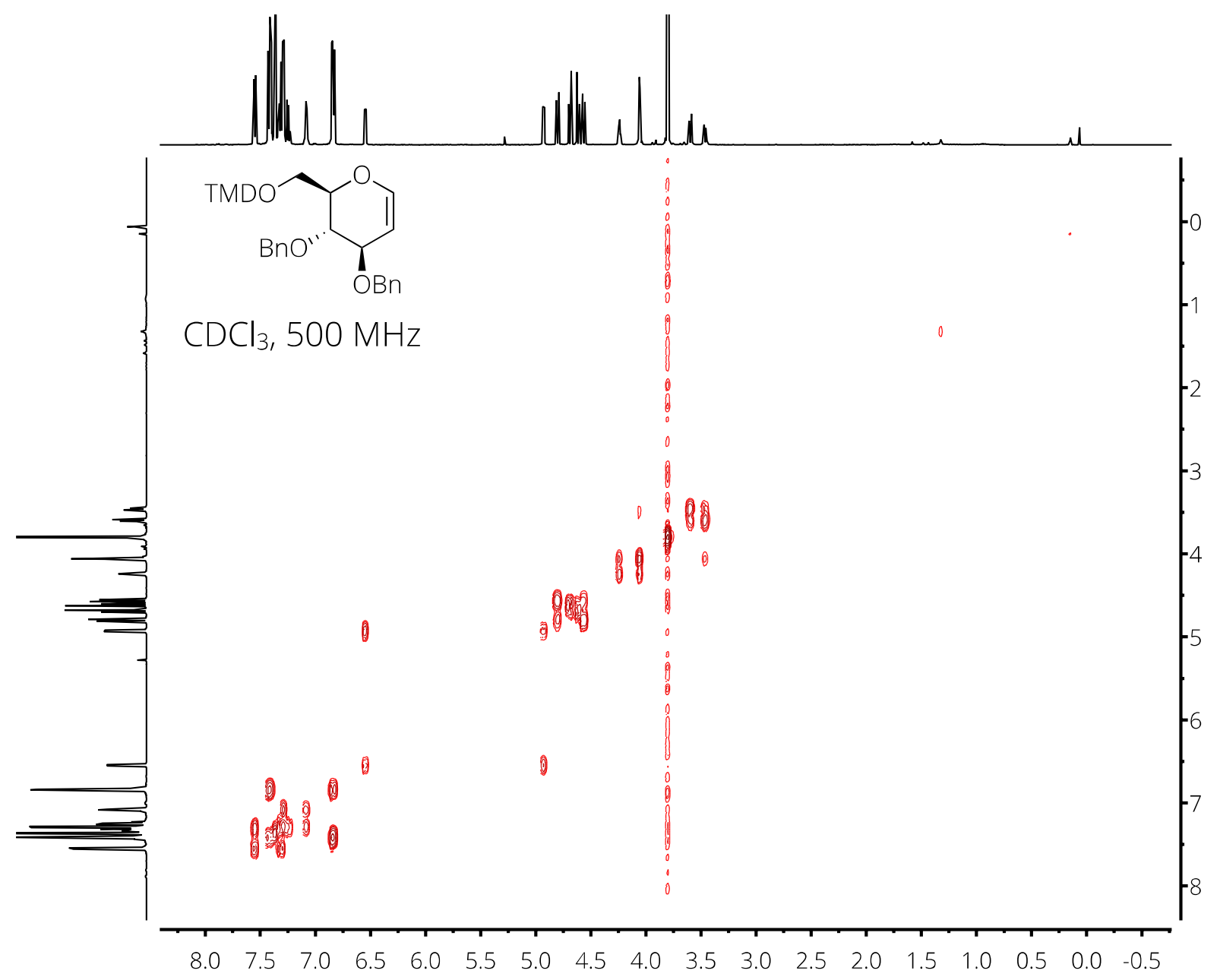




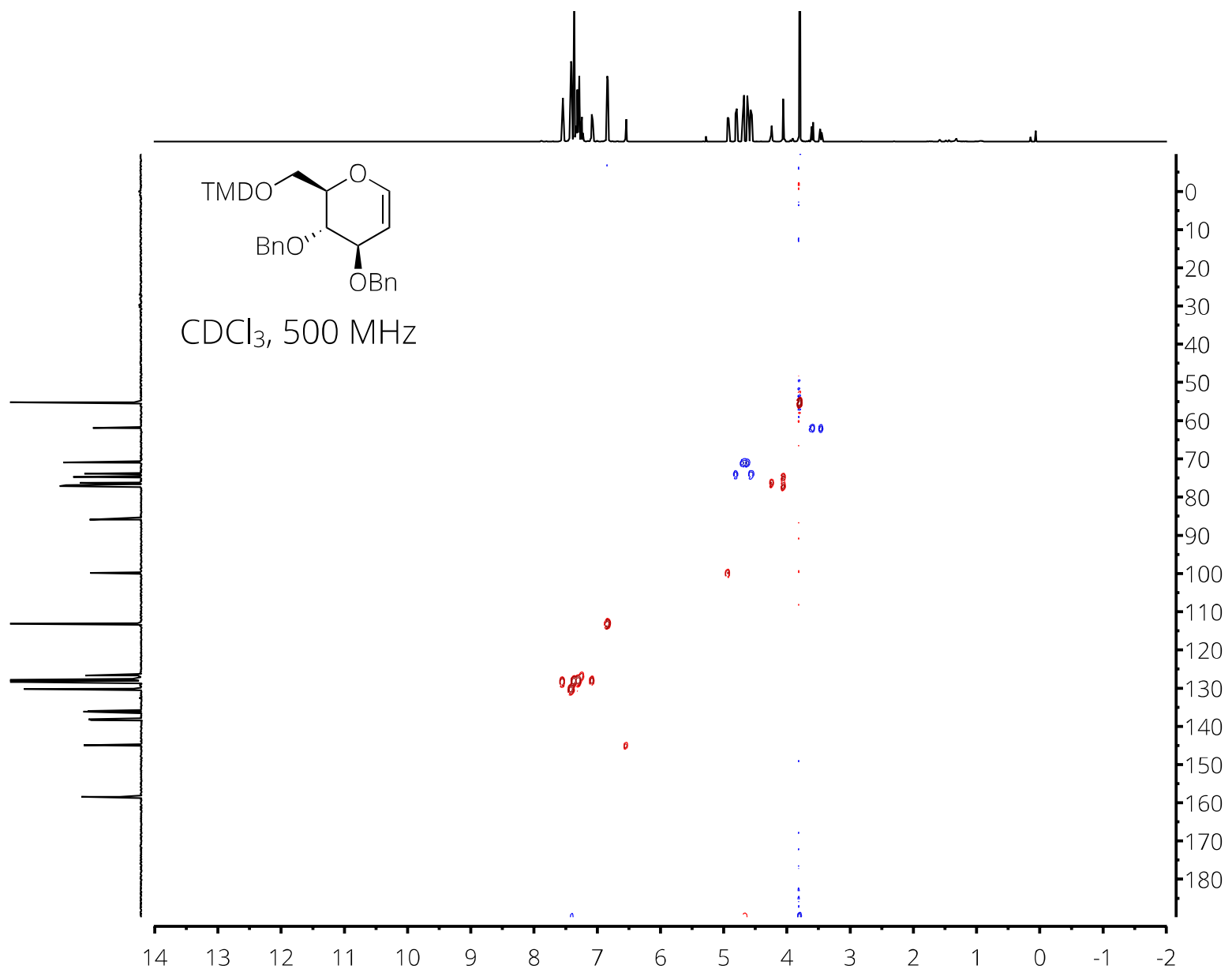




\section{Spectra of Cycloaddition Reaction Mixtures}

3,4,6-tri-O-acetyl-D-glucal (1) - ${ }^{1} \mathrm{H}-\mathrm{NMR}, \mathrm{CD}_{3} \mathrm{CN}, 500 \mathrm{MHz}$

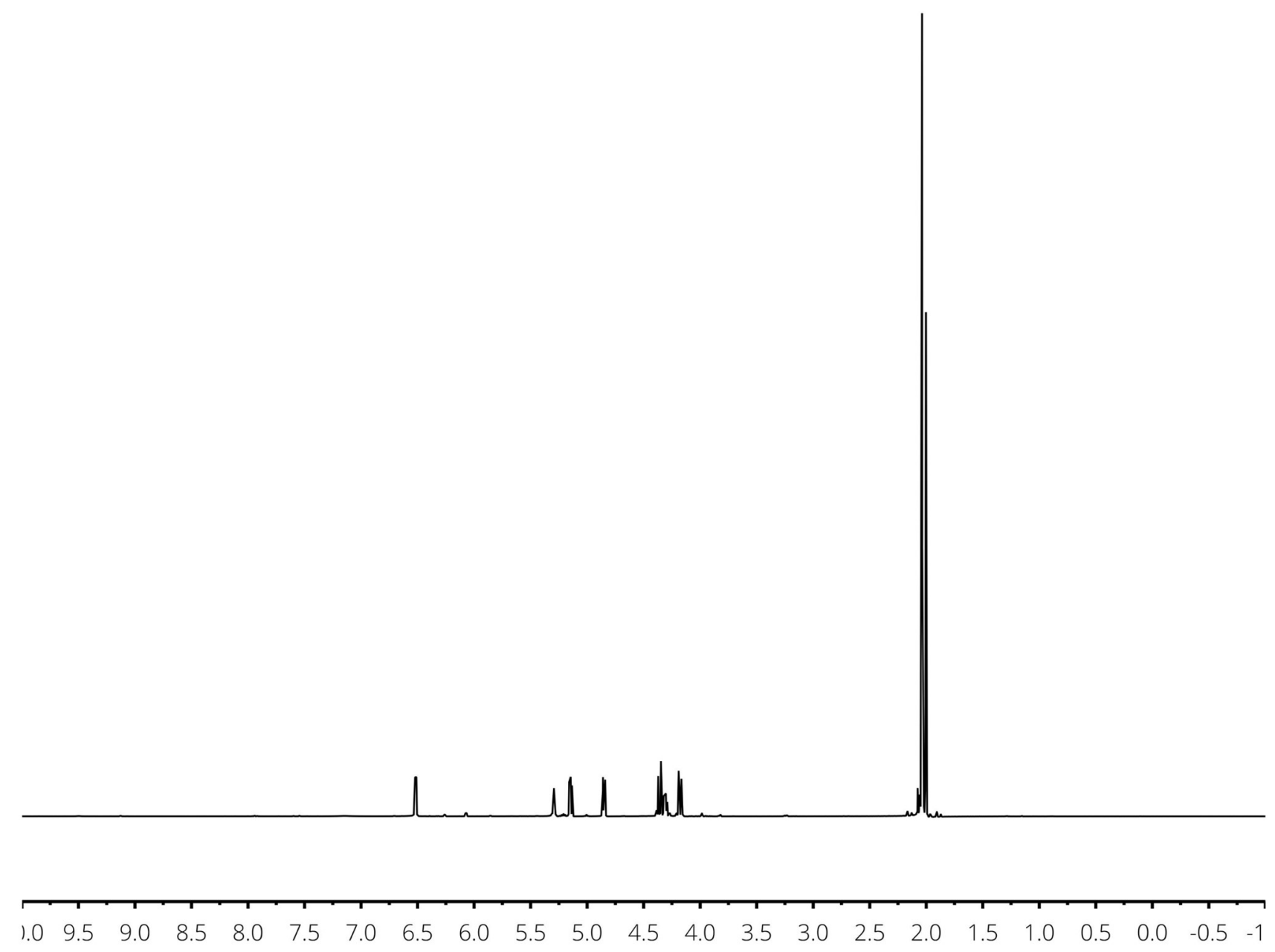



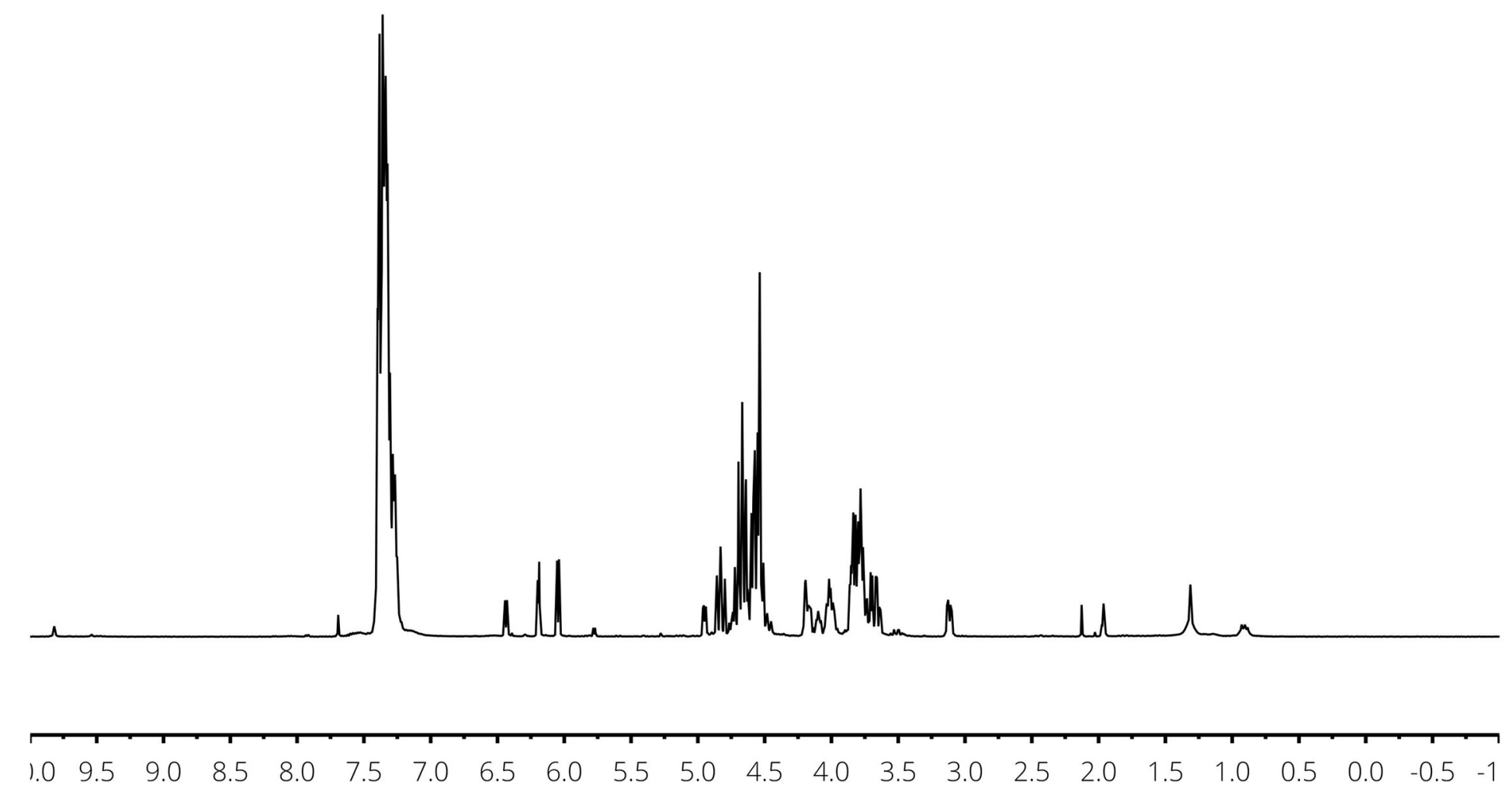
3,4,6-tri-O-methyl-D-glucal (3) - ${ }^{1} \mathrm{H}-\mathrm{NMR}, \mathrm{CD}_{3} \mathrm{CN}, 500 \mathrm{MHz}$

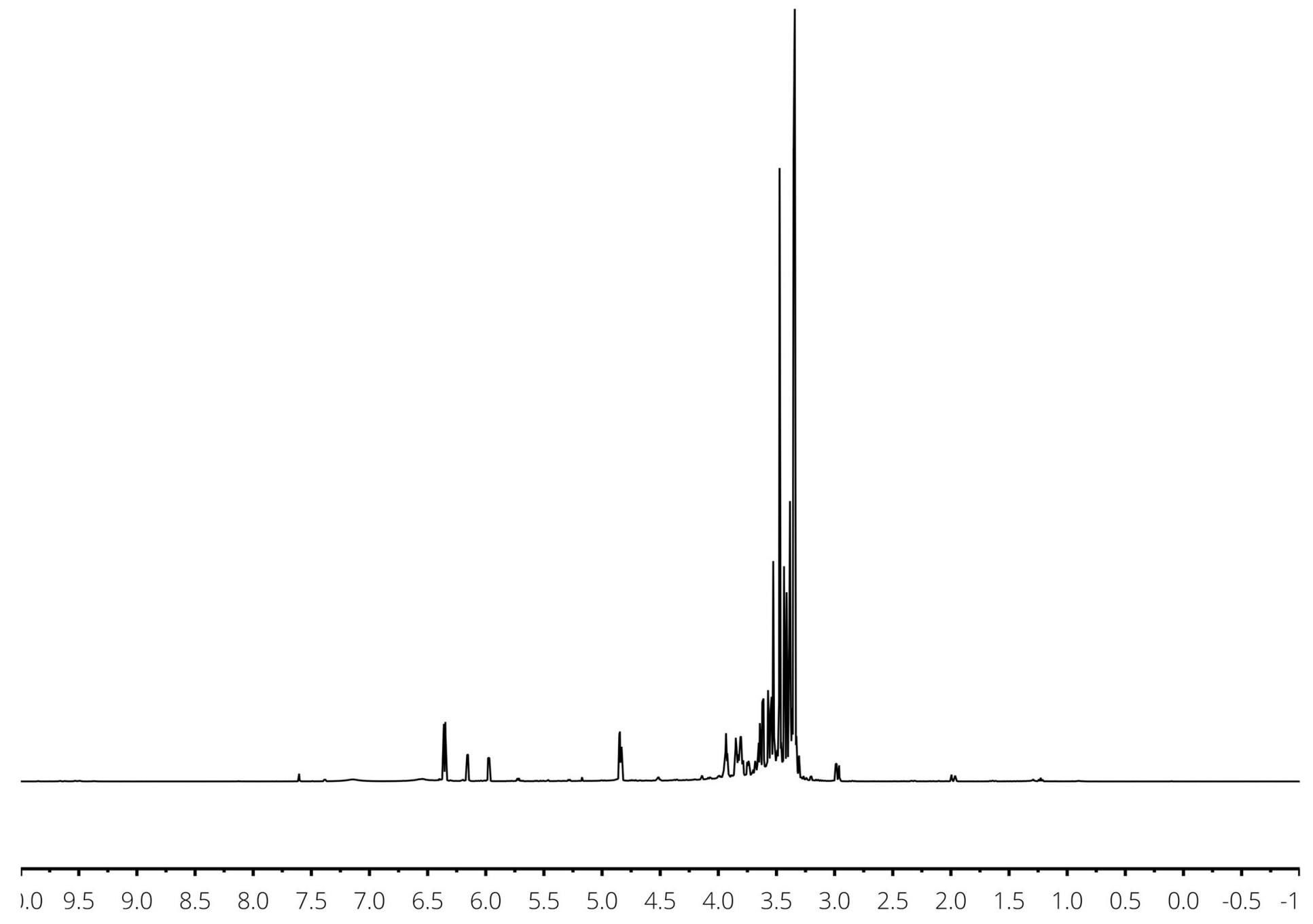


3,4,6-tri- $O$-ethyl-D-glucal (4) - ${ }^{1} \mathrm{H}-\mathrm{NMR}, \mathrm{CD}_{3} \mathrm{CN}, 500 \mathrm{MHz}$

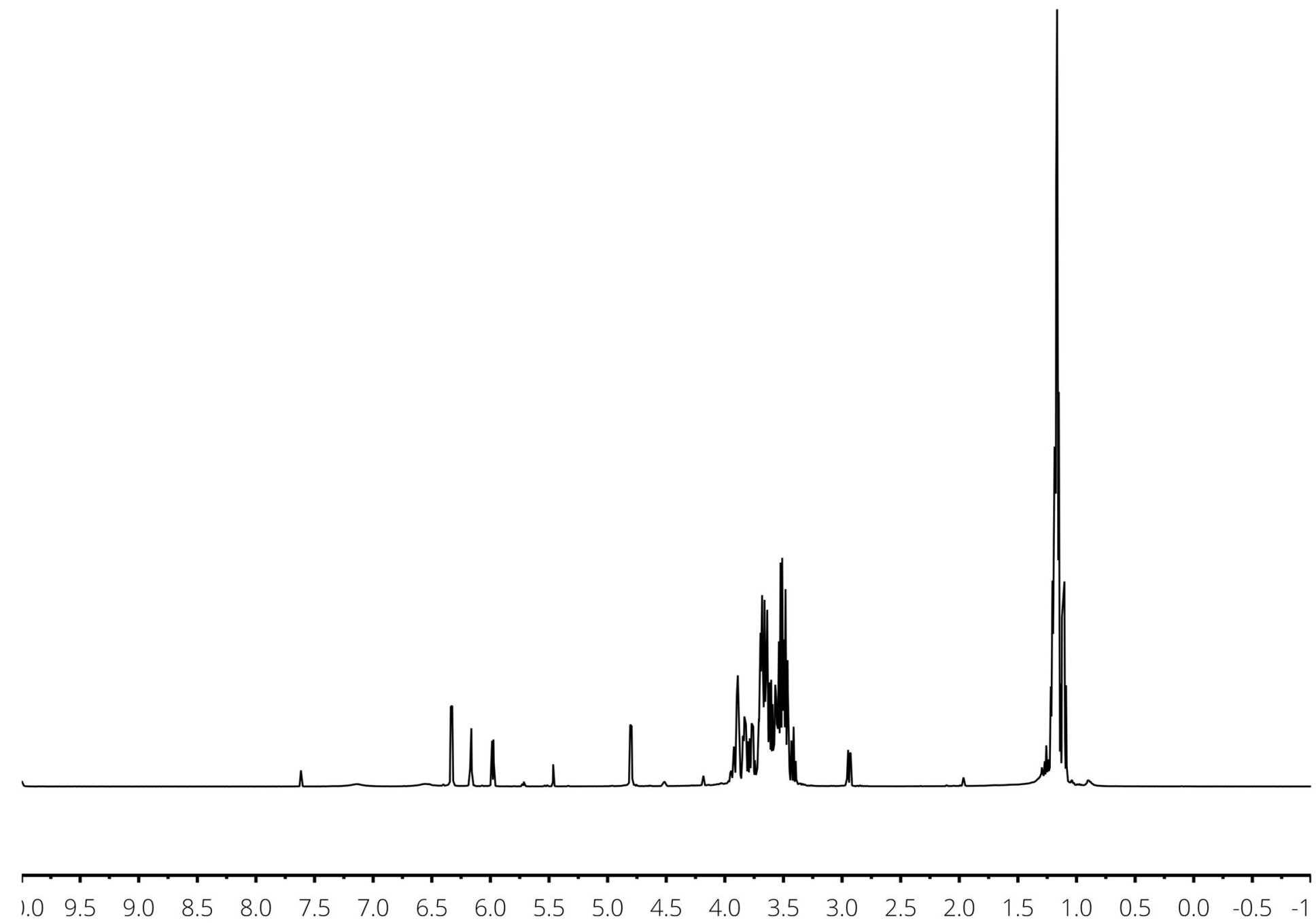


3,4,6-tri- $O$-triethylsilyl-D-glucal (5) - ${ }^{1} \mathrm{H}-\mathrm{NMR}, \mathrm{CD}_{3} \mathrm{CN}, 500 \mathrm{MHz}$

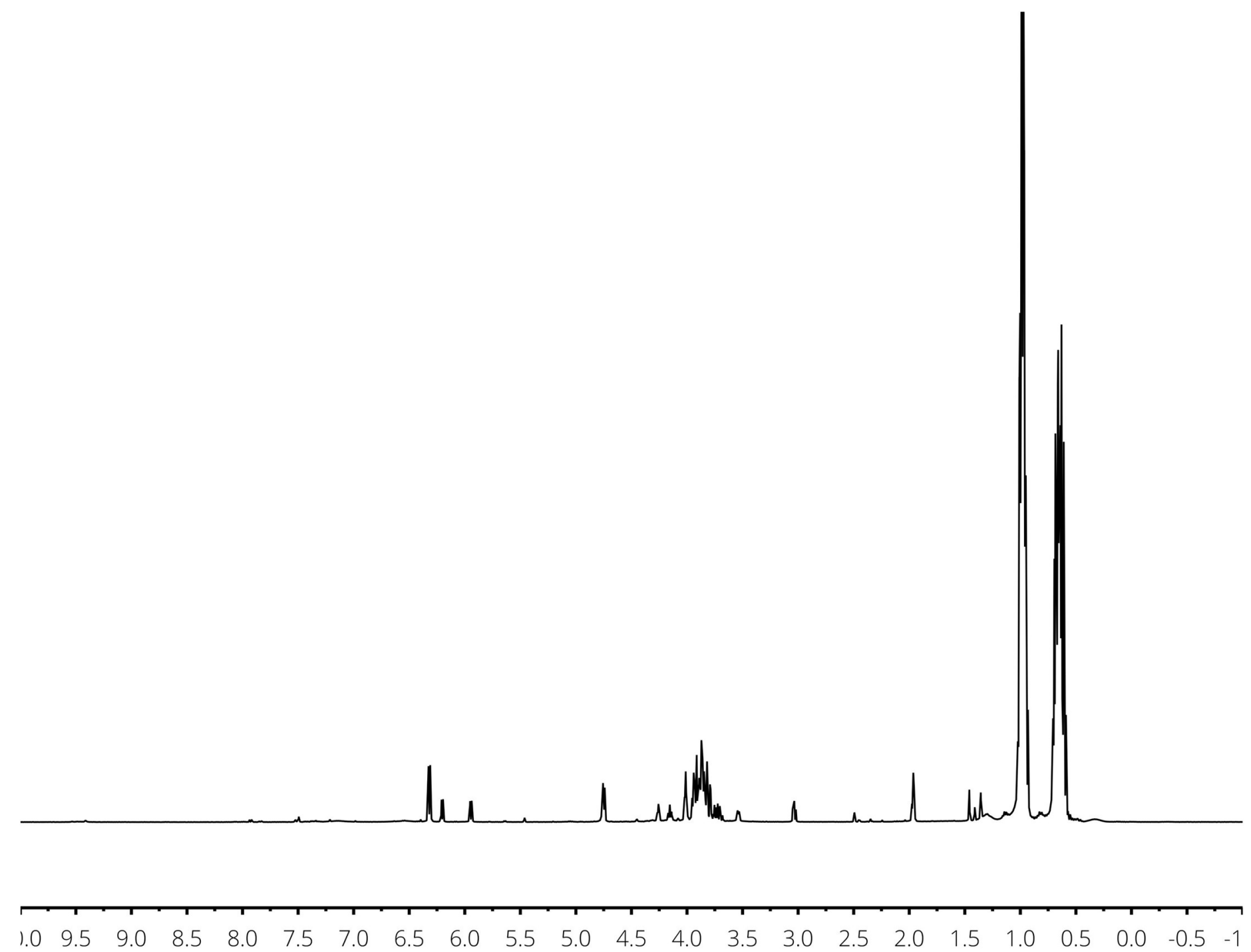


3,4,6-tri-O-p-methoxybenzyl-D-glucal (6) - ${ }^{1} \mathrm{H}-\mathrm{NMR}, \mathrm{CD}_{3} \mathrm{CN}, 500 \mathrm{MHz}$
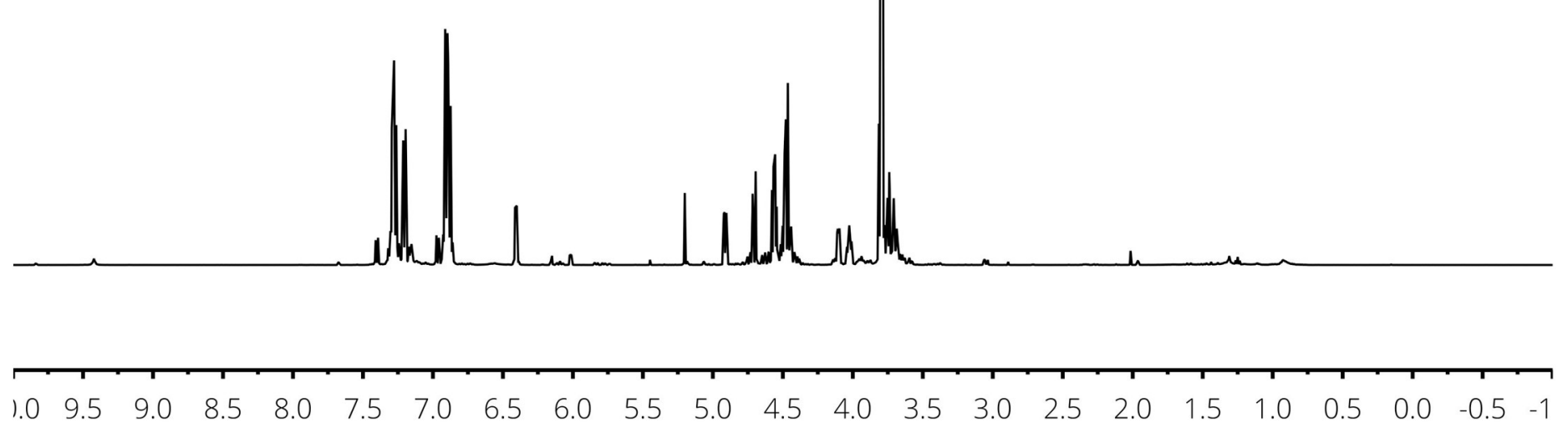

S

98 
3,4,6-tri-O-2,2,2-trichloroethylcarbonate-D-glucal (7) - ${ }^{1} \mathrm{H}-\mathrm{NMR}, \mathrm{CD}_{3} \mathrm{CN}, 500 \mathrm{MHz}$

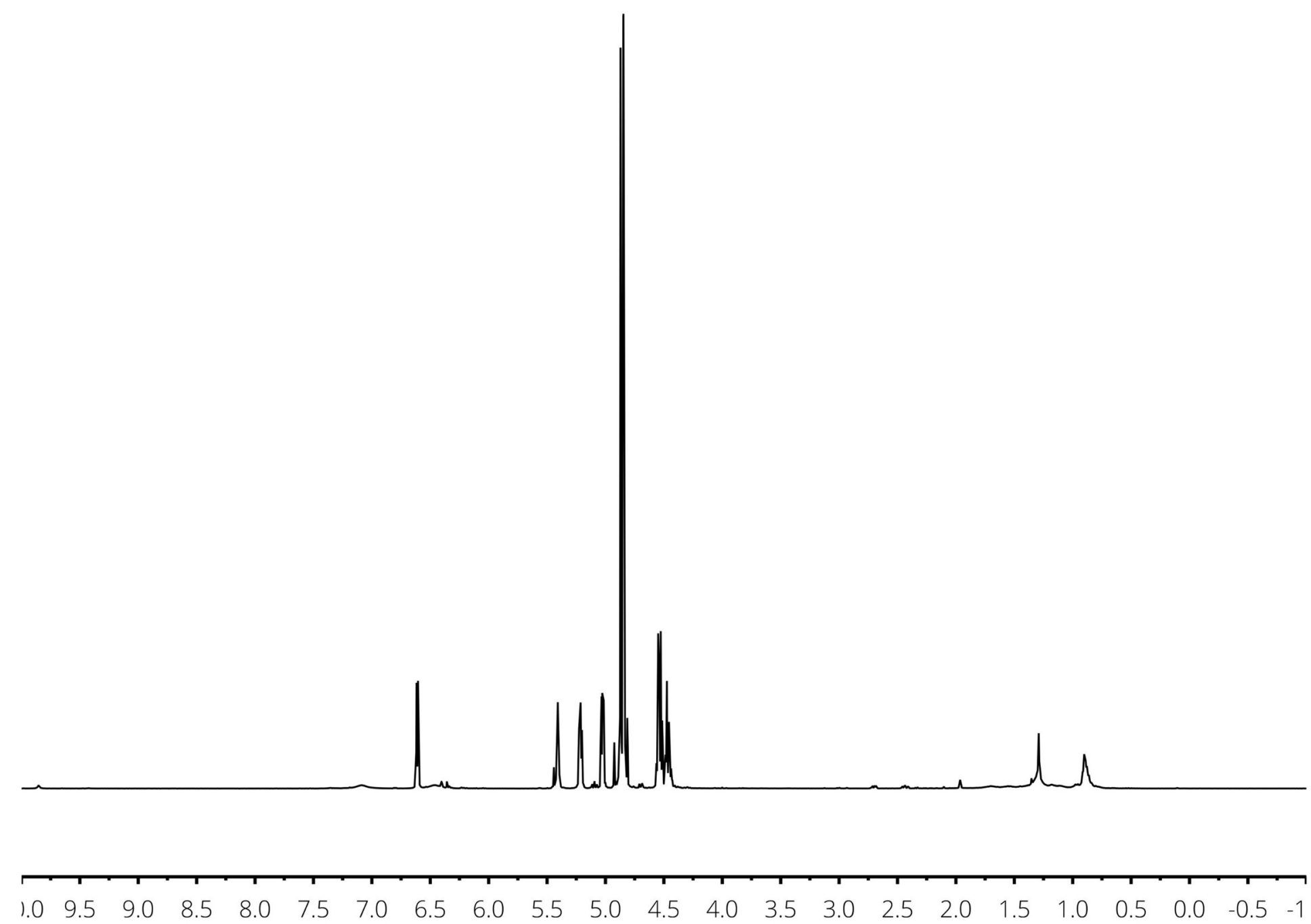



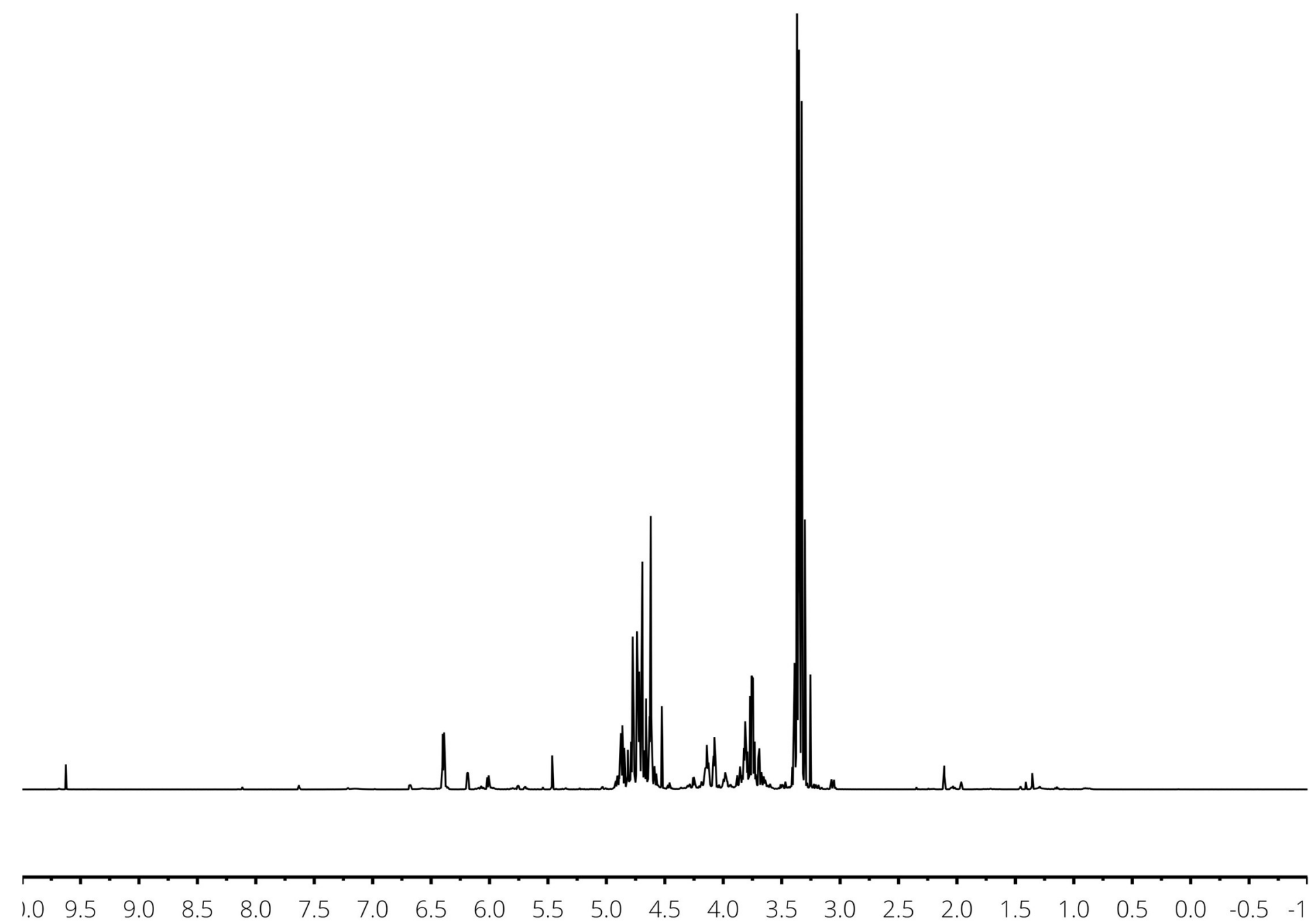
Benzyl 3,4-di-O-benzyl-D-glucuronal (9) - ${ }^{1} \mathrm{H}-\mathrm{NMR}, \mathrm{CD}_{3} \mathrm{CN}, 500 \mathrm{MHz}$
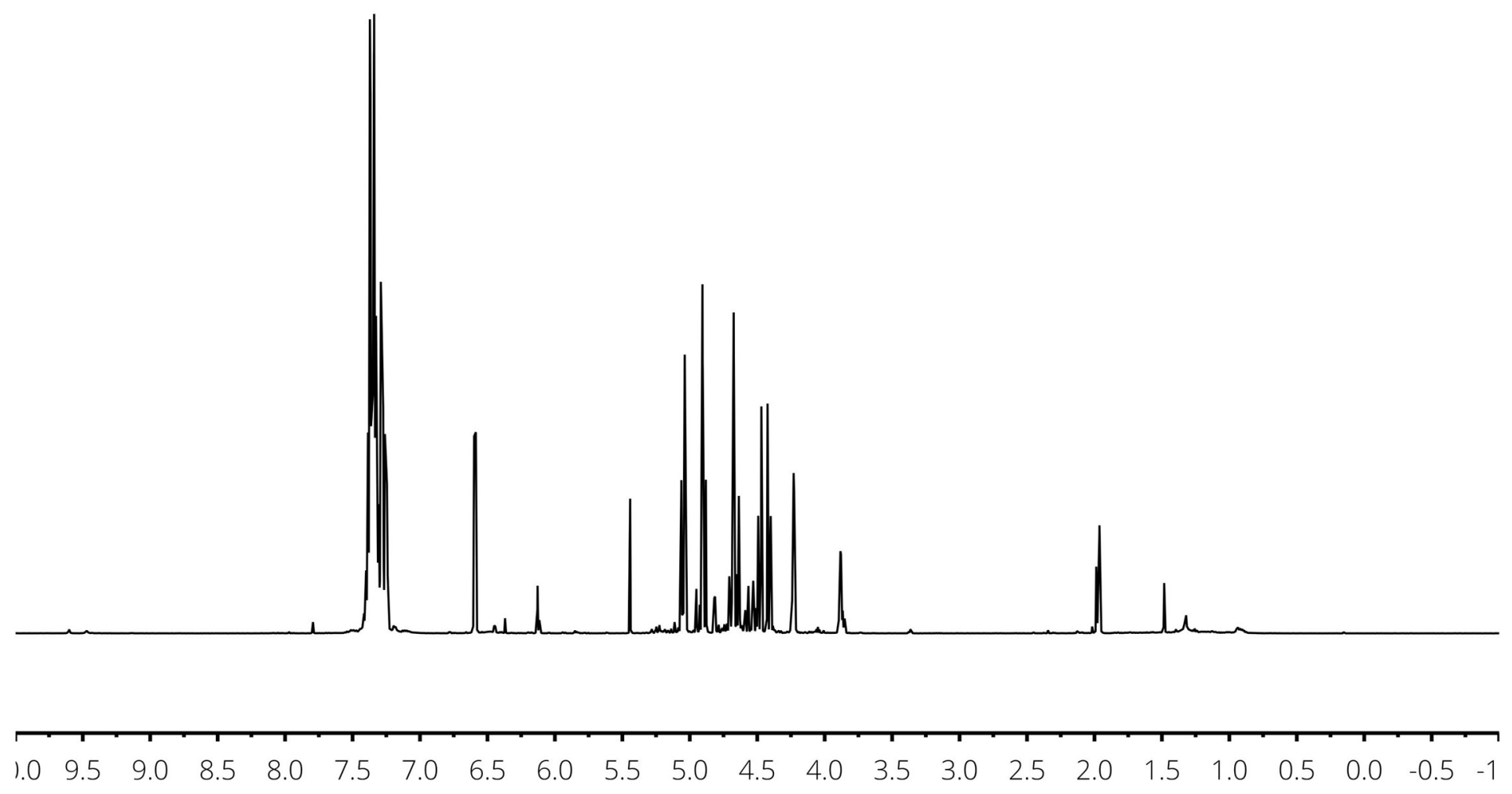


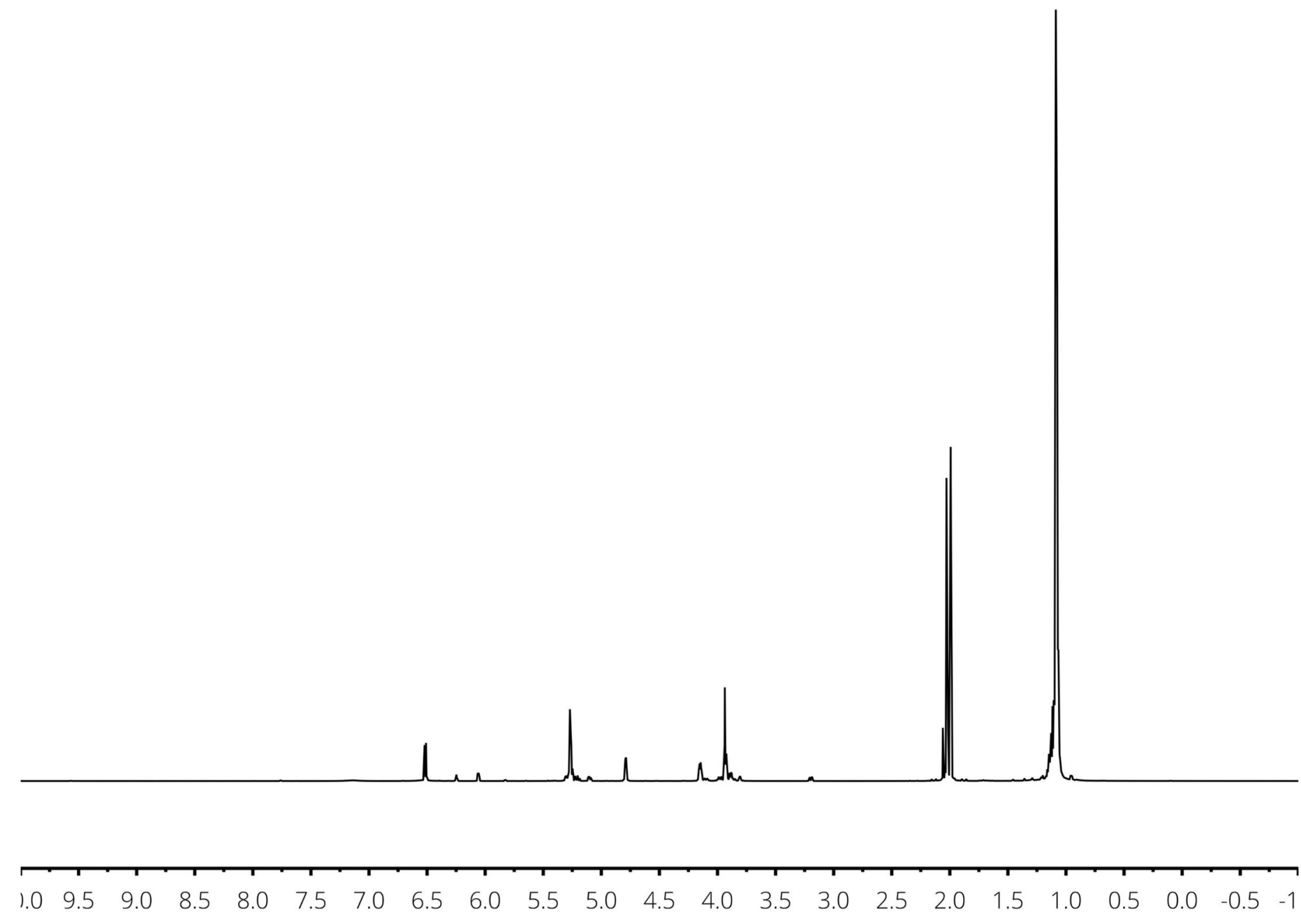




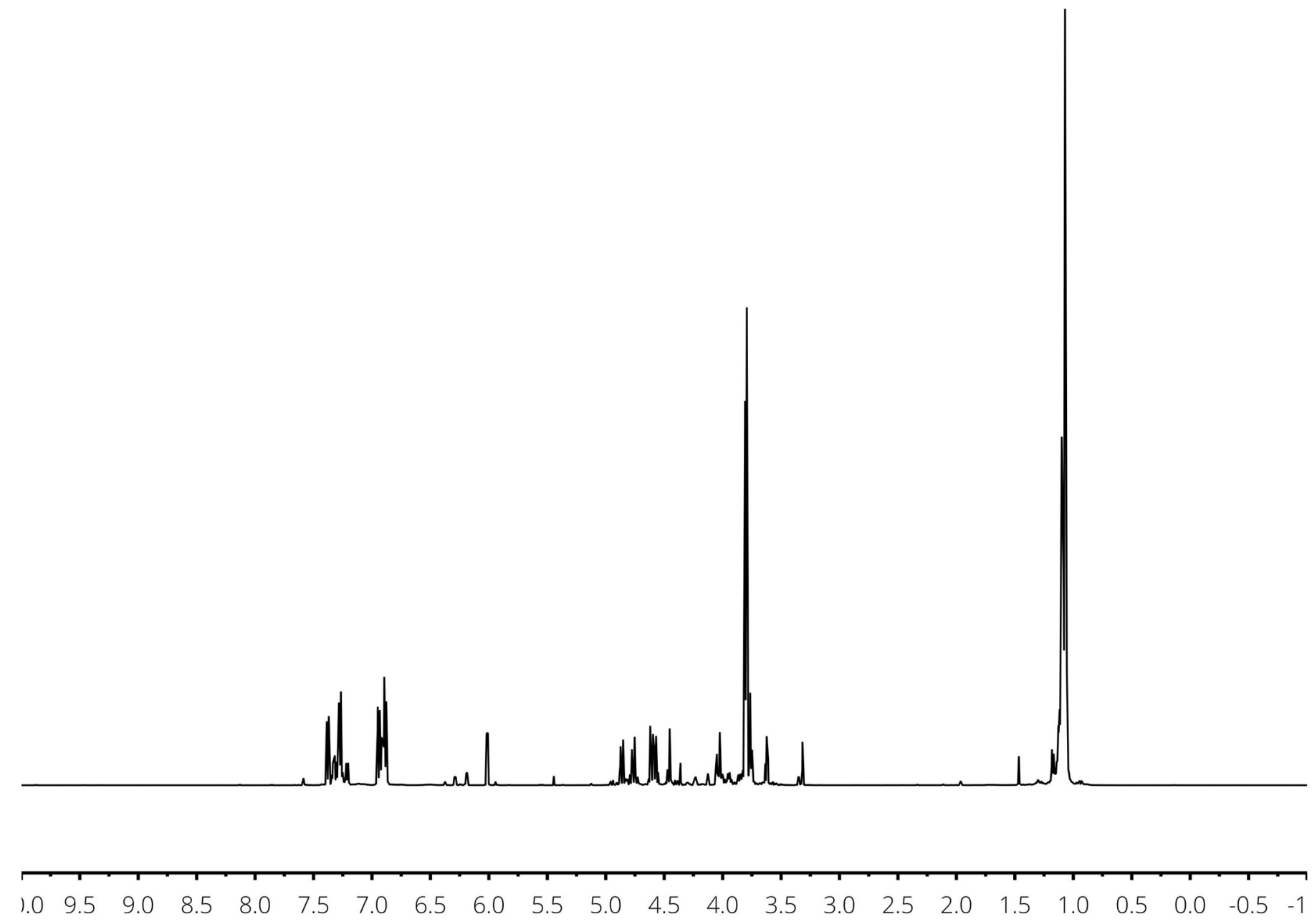




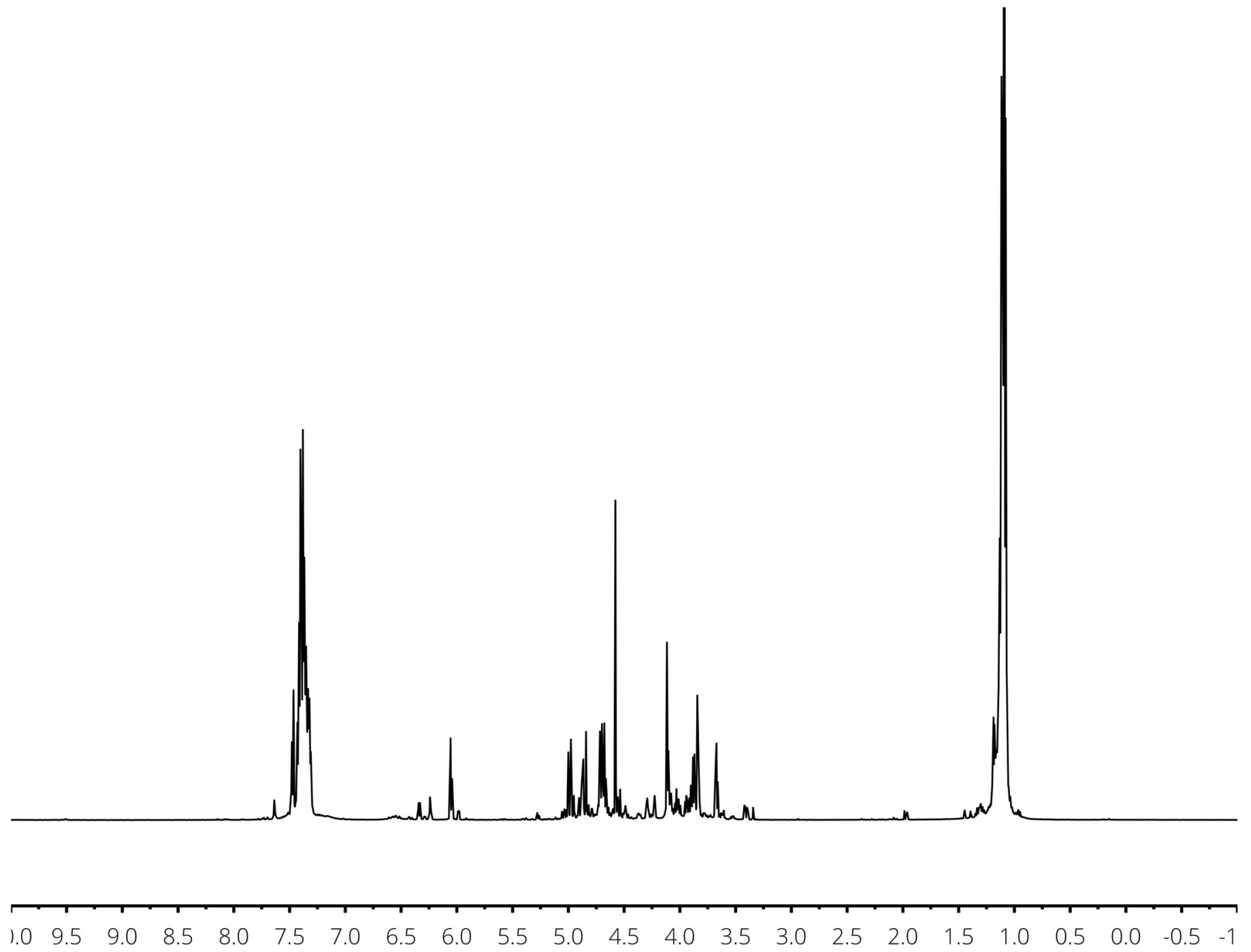




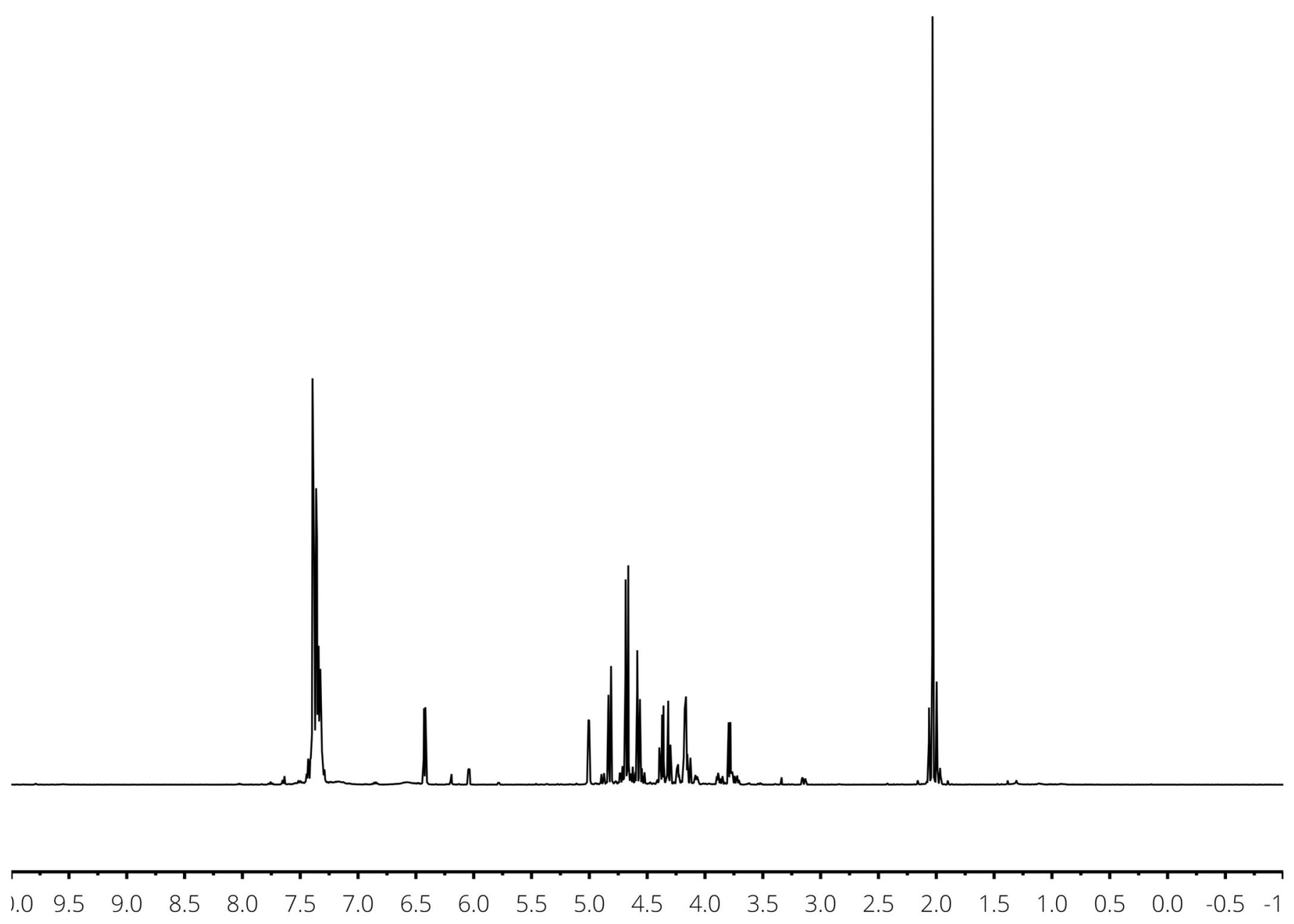



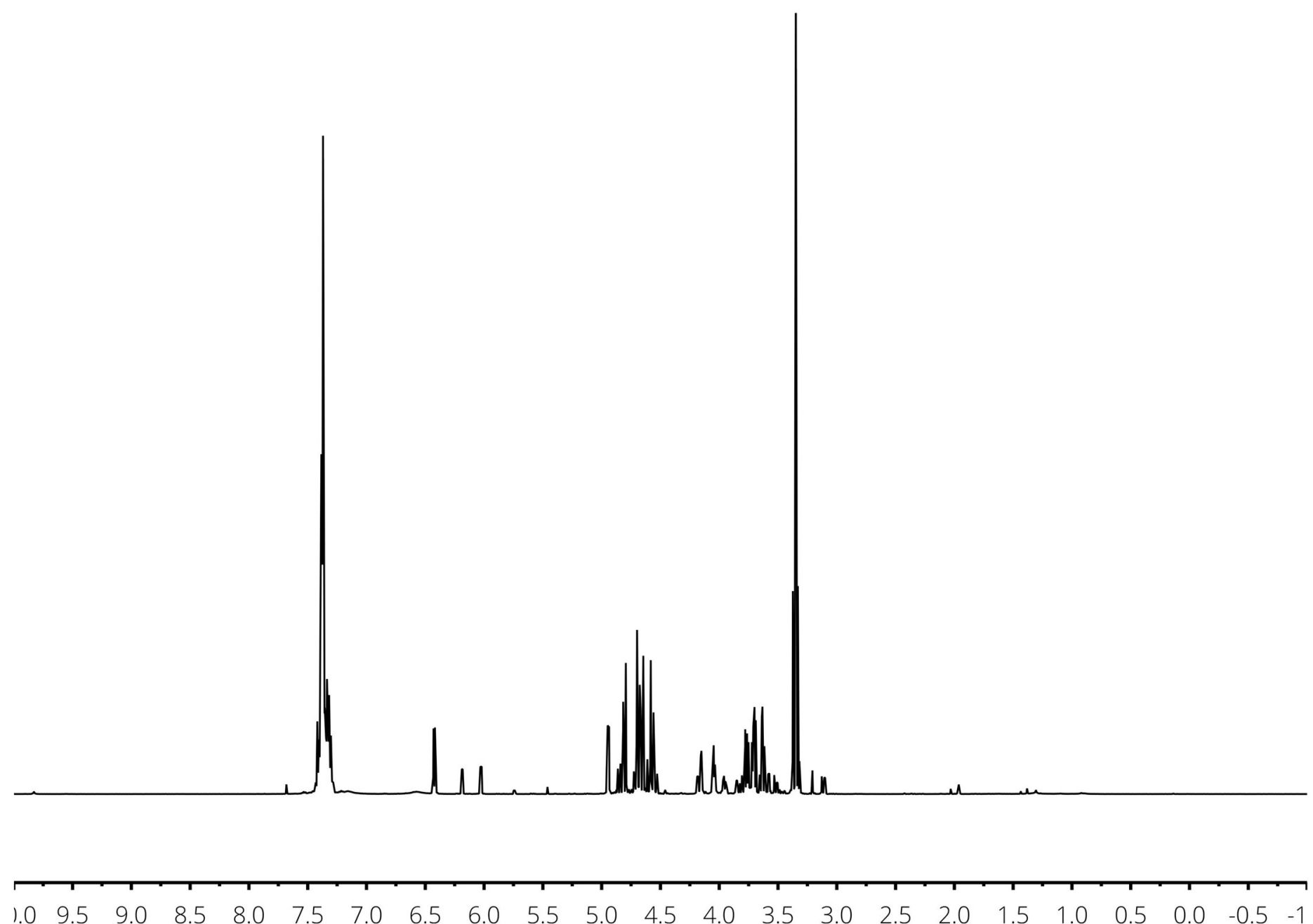
3,4-di-O-benzyl-6-O-2,2,2-trichloroethylcarbonate-D-glucal (14) - ${ }^{1} \mathrm{H}-\mathrm{NMR}, \mathrm{CD}_{3} \mathrm{CN}, 500 \mathrm{MHz}$

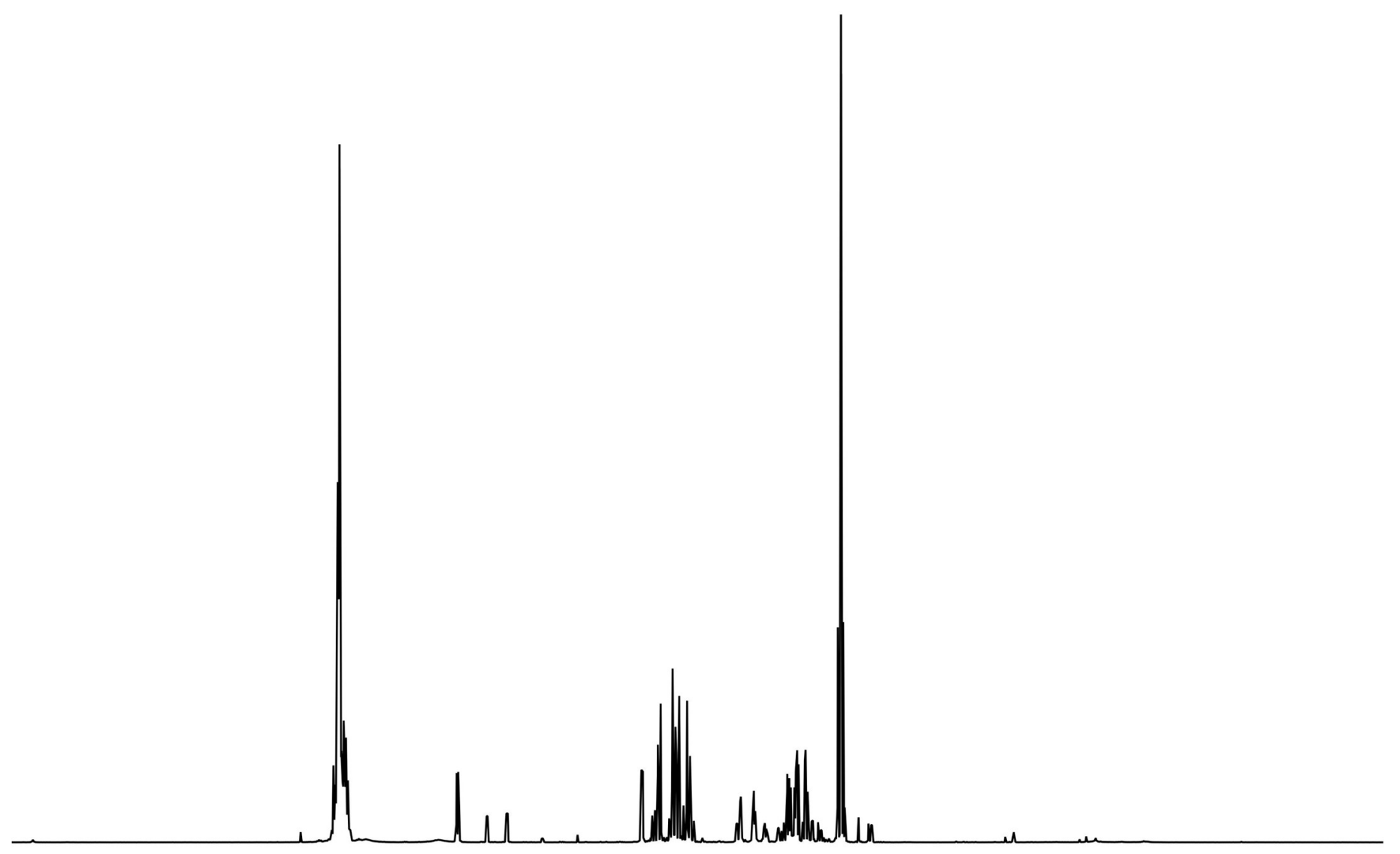

\begin{tabular}{lllllllllllllllllllllllll}
\hline .0 & 9.5 & 9.0 & 8.5 & 8.0 & 7.5 & 7.0 & 6.5 & 6.0 & 5.5 & 5.0 & 4.5 & 4.0 & 3.5 & 3.0 & 2.5 & 2.0 & 1.5 & 1.0 & 0.5 & 0.0 & -0.5 & -1
\end{tabular} 

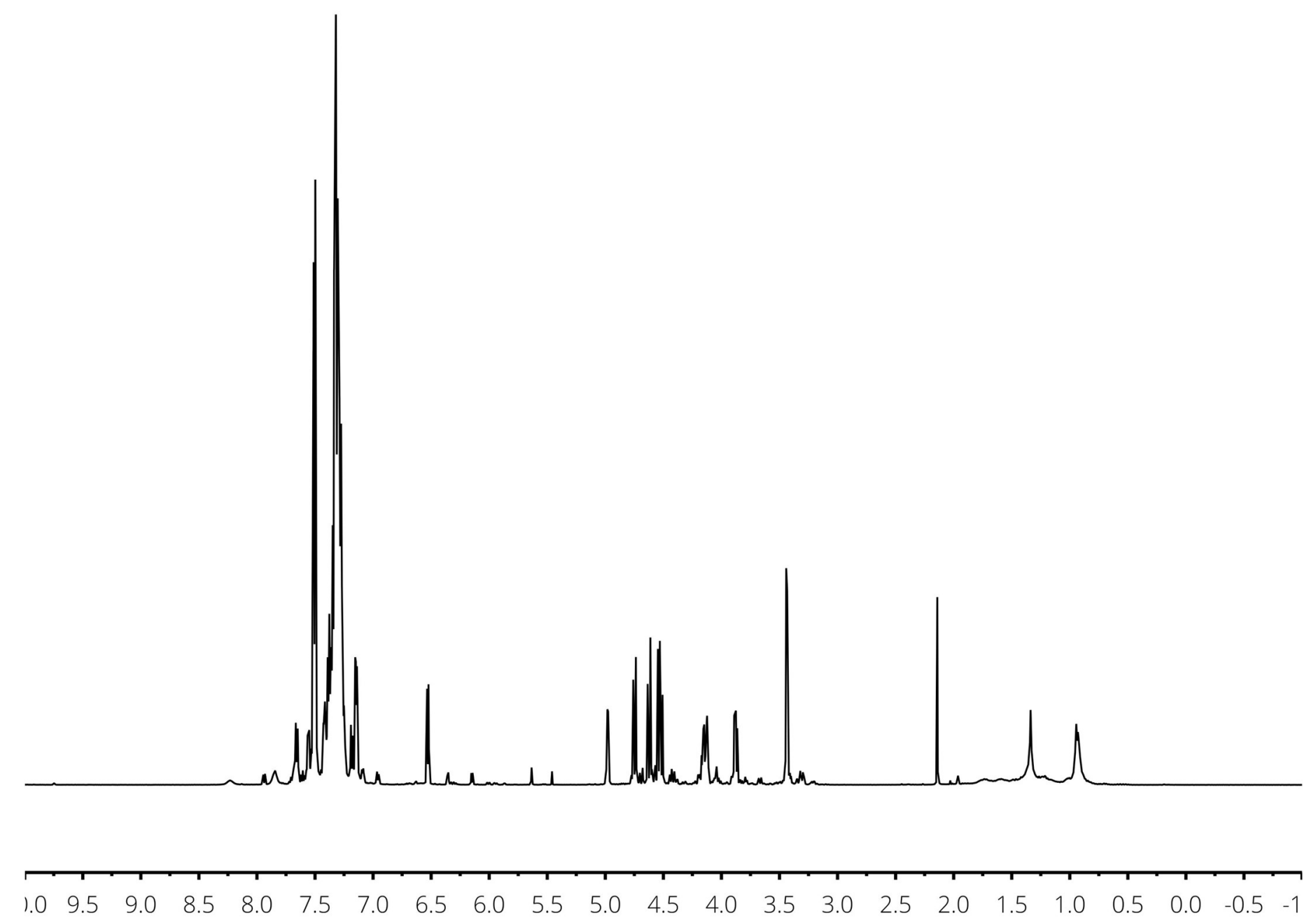


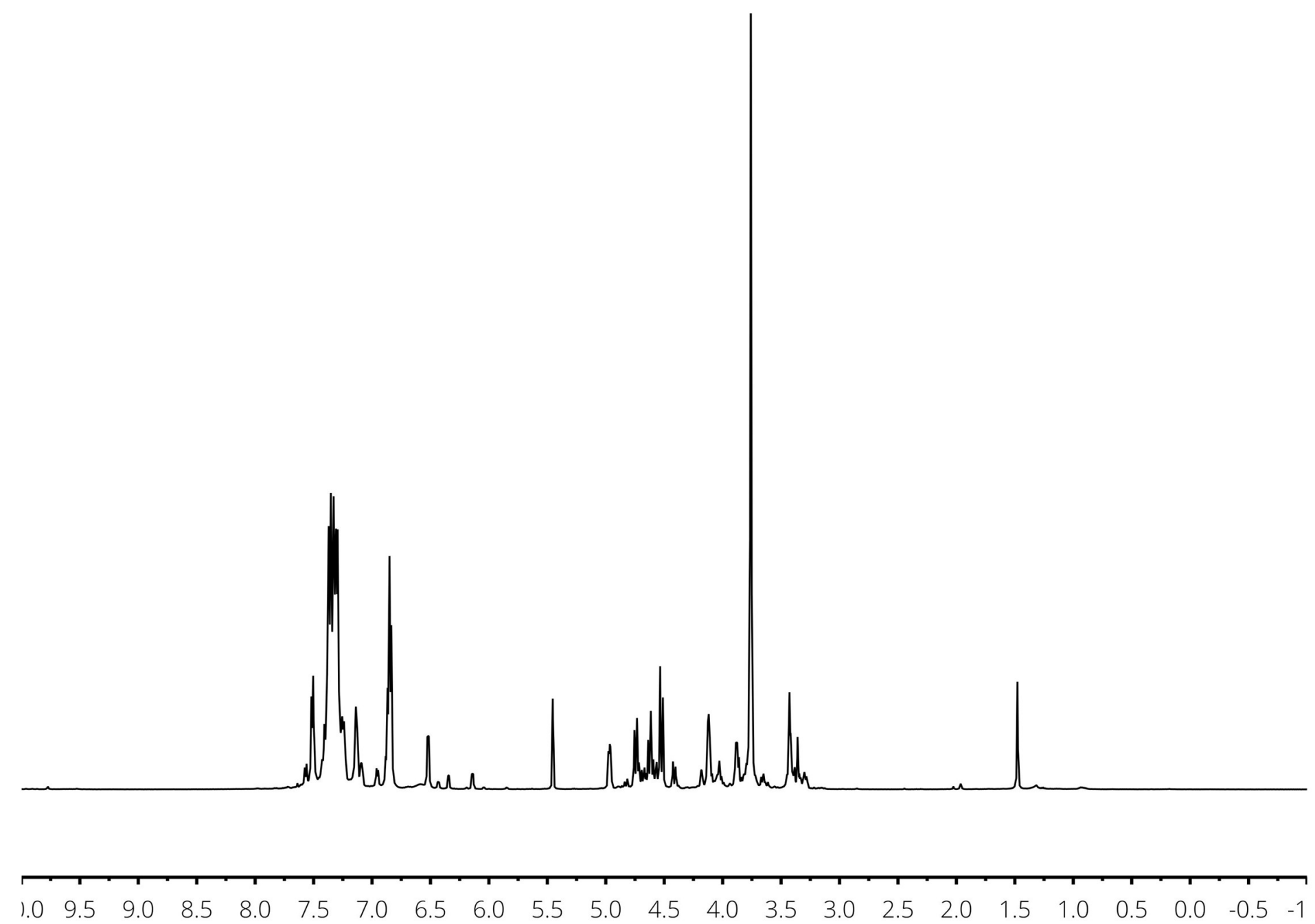




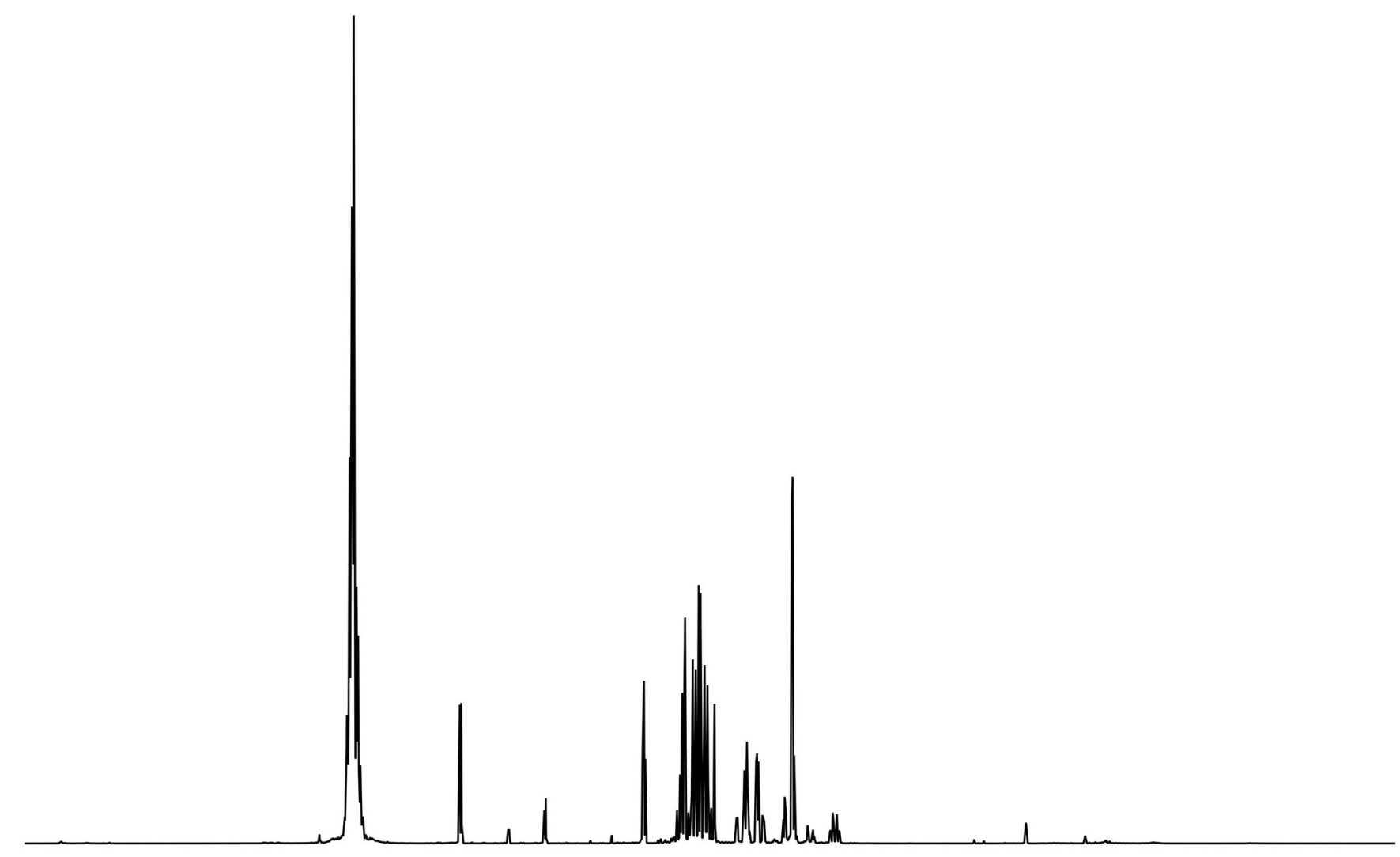

$\begin{array}{lllllllllllllllllllllll}1.0 & 9.5 & 9.0 & 8.5 & 8.0 & 7.5 & 7.0 & 6.5 & 6.0 & 5.5 & 5.0 & 4.5 & 4.0 & 3.5 & 3.0 & 2.5 & 2.0 & 1.5 & 1.0 & 0.5 & 0.0 & -0.5 & -1\end{array}$ 


\title{
Computational Results
}

\author{
Transition State Results
}

Table SCX1. Energetics of cycloaddition between TCAI and various substrates at the PBE0/DEF2-TZVP/CPCM $\left(\mathrm{CH}_{3} \mathrm{CH}\right) / \mathrm{D} 3 \mathrm{BJ} / / \mathrm{PBE} 0 / \mathrm{DEF} 2-\mathrm{SVP} / \mathrm{CPCM}\left(\mathrm{CH}_{3} \mathrm{CN}\right) / \mathrm{D} 3 \mathrm{BJ}$ level.

\begin{tabular}{|c|c|c|c|c|c|c|}
\hline & Conformer & $\mathbf{E}$ (a.u.) & $\mathbf{G}$ (a.u.) & $\begin{array}{c}\Delta \mathbf{E}^{(\mathbf{i})} \\
(\mathbf{k c a l} / \mathbf{m o l})\end{array}$ & $\begin{array}{c}\Delta \mathbf{G} \\
(\mathbf{k c a l} / \mathbf{m o l})\end{array}$ & $\boldsymbol{\varepsilon}^{(\mathbf{i i})} \mathbf{( e V )}$ \\
\hline $\mathbf{1}$ & ${ }^{5} \mathrm{H}_{4}$ & -2692.3051 & -2692.0513 & 12.5 & 28.2 & -8.222 \\
\hline $\mathbf{3}$ & ${ }^{5} \mathrm{H}_{4}$ & -2352.4214 & -2352.1900 & 10.9 & 26.3 & -7.995 \\
\hline $\mathbf{4}$ & ${ }^{5} \mathrm{H}_{4}$ & -2470.2812 & -2469.9681 & 9.6 & 25.5 & -8.006 \\
\hline $\mathbf{1 2}$ & ${ }^{5} \mathrm{H}_{4}$ & -3419.0860 & -3418.4658 & 10.6 & 27.4 & -8.023 \\
\hline $\mathbf{1 5}$ & ${ }^{5} \mathrm{H}_{4}$ & -4420.3171 & -4419.9208 & 12.3 & 27.2 & -8.133 \\
\hline $\mathbf{1}$ & ${ }^{4} \mathrm{H}_{5}$ & -2692.3115 & -2692.0563 & 8.0 & 24.2 & -8.371 \\
\hline $\mathbf{3}$ & ${ }^{4} \mathrm{H}_{5}$ & -2352.4292 & -2352.1983 & 6.2 & 21.6 & -8.109 \\
\hline $\mathbf{4}$ & ${ }^{4} \mathrm{H}_{5}$ & -2470.2874 & -2469.9760 & 5.9 & 21.6 & -8.088 \\
\hline $\mathbf{1 2}$ & ${ }^{4} \mathrm{H}_{5}$ & -3419.0953 & -3418.4741 & 2.2 & 19.4 & -8.141 \\
\hline $\mathbf{1 5}$ & ${ }^{4} \mathrm{H}_{5}$ & -4420.3232 & -4419.9278 & 6.9 & 22.7 & -8.168 \\
\hline
\end{tabular}

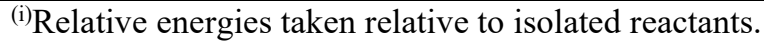

(ii)NBO orbital energy isn't corrected for delocalization.

\section{Energetics for Sampled Conformations}

Table SCY1. Energetics for 1 at the PBE0/D3BJ/CPCM $\left(\mathrm{CH}_{3} \mathrm{CN}\right) / / \mathrm{PBE} 0 / \mathrm{D} 3 \mathrm{BJ} / \mathrm{DEF} 2-$ $\mathrm{SVP} / \mathrm{RIJCOSX}$ level. Relative energies in $\mathrm{kcal} / \mathrm{mol}$.

\begin{tabular}{|c|c|c|c|c|c|c|c|c|}
\hline E (a.u.) & G (a.u.) & $\Delta \mathrm{G}$ & Boltz. & $\begin{array}{l}\text { HOMO } \\
(\mathrm{eV})\end{array}$ & $\begin{array}{c}\pi_{C_{1}-C_{2}} \\
\quad(\mathrm{ev})\end{array}$ & $\begin{array}{c}\Delta E^{(2)}(e V) \\
\left(\pi \rightarrow \sigma_{C_{3}-o}^{*}\right)\end{array}$ & $\begin{array}{c}\Delta E^{(2)}(e V) \\
\left(\pi \rightarrow \sigma_{C_{3}-H}^{*}\right)\end{array}$ & Conformer \\
\hline-992.8365 & -992.6025 & 1.40 & 0.095 & -7.057 & -8.243 & 0.346 & 0.093 & ${ }^{5} \mathrm{H}_{4}$ \\
\hline-992.8373 & -992.6047 & 0.00 & 1.000 & -7.047 & -8.228 & 0.353 & 0.090 & ${ }^{5} \mathbf{H}_{4}$ \\
\hline-992.8369 & -992.6040 & 0.44 & 0.473 & -7.044 & -8.242 & 0.388 & 0.085 & ${ }^{5} \mathrm{H}_{4}$ \\
\hline-992.8369 & -992.6041 & 0.41 & 0.501 & -7.022 & -8.250 & 0.353 & 0.097 & ${ }^{5} \mathrm{H}_{4}$ \\
\hline-992.8343 & -992.5998 & 3.06 & 0.006 & -6.984 & -8.243 & 0.346 & 0.097 & ${ }^{5} \mathrm{H}_{4}$ \\
\hline-992.8377 & -992.6034 & 0.85 & 0.239 & -6.946 & -8.331 & 0.212 & 0.160 & ${ }^{4} \mathrm{H}_{5}$ \\
\hline-992.8338 & -992.5996 & 3.23 & 0.004 & -6.915 & -8.352 & 0.214 & 0.154 & ${ }^{4} \mathrm{H}_{5}$ \\
\hline-992.8314 & -992.5980 & 4.19 & 0.001 & -6.933 & -8.378 & 0.221 & 0.149 & ${ }^{4} \mathrm{H}_{5}$ \\
\hline-992.8362 & -992.6018 & 1.81 & 0.047 & -7.027 & -8.229 & 0.360 & 0.087 & ${ }^{5} \mathrm{H}_{4}$ \\
\hline-992.8377 & -992.6034 & 0.80 & 0.260 & -6.946 & -8.331 & 0.212 & 0.160 & ${ }^{4} \mathrm{H}_{5}$ \\
\hline-992.8362 & -992.6037 & 0.62 & 0.351 & -6.955 & -8.350 & 0.209 & 0.161 & ${ }^{4} \mathrm{H}_{5}$ \\
\hline-992.8341 & -992.6019 & 1.78 & 0.050 & -6.962 & -8.357 & 0.212 & 0.160 & ${ }^{4} \mathrm{H}_{5}$ \\
\hline-992.8334 & -992.6007 & 2.54 & 0.014 & -6.930 & -8.378 & 0.221 & 0.150 & ${ }^{4} \mathrm{H}_{5}$ \\
\hline-992.8339 & -992.5996 & 3.18 & 0.005 & -6.914 & -8.353 & 0.212 & 0.154 & ${ }^{4} \mathrm{H}_{5}$ \\
\hline-992.8367 & -992.6034 & 0.85 & 0.237 & -6.935 & -8.336 & 0.205 & 0.161 & ${ }^{4} \mathrm{H}_{5}$ \\
\hline-992.8355 & -992.6022 & 1.60 & 0.067 & -6.948 & -8.344 & 0.205 & 0.161 & ${ }^{4} \mathrm{H}_{5}$ \\
\hline-992.8338 & -992.6002 & 2.85 & 0.008 & -6.950 & -8.353 & 0.212 & 0.160 & ${ }^{4} \mathrm{H}_{5}$ \\
\hline-992.8324 & -992.5995 & 3.24 & 0.004 & -6.927 & -8.374 & 0.218 & 0.152 & ${ }^{4} \mathrm{H}_{5}$ \\
\hline-992.8334 & -992.5999 & 3.05 & 0.006 & -6.909 & -8.370 & 0.218 & 0.152 & ${ }^{4} \mathrm{H}_{5}$ \\
\hline-992.8300 & -992.5956 & 5.71 & 0.000 & -6.916 & -8.367 & 0.214 & 0.154 & ${ }^{4} \mathrm{H}_{5}$ \\
\hline-992.8326 & -992.5978 & 4.31 & 0.001 & -6.938 & -8.377 & 0.217 & 0.151 & ${ }^{4} \mathrm{H}_{5}$ \\
\hline
\end{tabular}


$\begin{array}{lllllllll}-992.8359 & -992.6025 & 1.36 & 0.100 & -6.944 & -8.337 & 0.211 & 0.160 & { }^{4} \mathrm{H}_{5}\end{array}$

Table SCY2. Energetics for 2 at the PBE0/D3BJ/CPCM $\left(\mathrm{CH}_{3} \mathrm{CN}\right) / / \mathrm{PBE} 0 / \mathrm{D} 3 \mathrm{BJ} / \mathrm{DEF} 2-$ $\mathrm{SVP} / \mathrm{RIJCOSX}$ level. Relative energies in $\mathrm{kcal} / \mathrm{mol}$.

\begin{tabular}{|c|c|c|c|c|c|c|c|c|}
\hline E (a.u.) & G (a.u.) & $\Delta \mathrm{G}$ & Boltz. & $\begin{array}{l}\text { HOMO } \\
(\mathrm{eV})\end{array}$ & $\begin{array}{c}\pi_{C_{1}-C_{2}} \\
\quad(\mathrm{ev})\end{array}$ & $\begin{array}{c}\Delta E^{(2)}(e V) \\
\left(\pi \rightarrow \sigma_{C_{3}-O}^{*}\right)\end{array}$ & $\begin{array}{c}\Delta E^{(2)}(e V) \\
\left(\pi \rightarrow \sigma_{C_{3}-H}^{*}\right)\end{array}$ & Conformer \\
\hline-1345.5702 & -1345.1277 & 3.18 & 0.005 & -6.856 & -8.055 & 0.295 & 0.095 & ${ }^{5} \mathrm{H}_{4}$ \\
\hline-1345.5729 & -1345.1291 & 2.27 & 0.022 & -6.714 & -8.005 & 0.306 & 0.095 & ${ }^{5} \mathrm{H}_{4}$ \\
\hline-1345.5721 & -1345.1289 & 2.39 & 0.018 & -6.687 & -7.990 & 0.323 & 0.091 & ${ }^{5} \mathrm{H}_{4}$ \\
\hline-1345.5732 & -1345.1293 & 2.16 & 0.026 & -6.657 & -7.970 & 0.347 & 0.077 & ${ }^{5} \mathrm{H}_{4}$ \\
\hline-1345.5724 & -1345.1291 & 2.27 & 0.022 & -6.690 & -7.993 & 0.323 & 0.091 & ${ }^{5} \mathrm{H}_{4}$ \\
\hline-1345.5768 & -1345.1322 & 0.34 & 0.564 & -6.778 & -8.009 & 0.301 & 0.102 & ${ }^{5} \mathrm{H}_{4}$ \\
\hline-1345.5725 & -1345.1298 & 1.83 & 0.046 & -6.797 & -8.041 & 0.308 & 0.100 & ${ }^{5} \mathrm{H}_{4}$ \\
\hline-1345.5743 & -1345.1308 & 1.20 & 0.131 & -6.798 & -8.041 & 0.317 & 0.091 & ${ }^{5} \mathrm{H}_{4}$ \\
\hline-1345.5739 & -1345.1302 & 1.57 & 0.070 & -6.784 & -8.058 & 0.322 & 0.099 & ${ }^{5} \mathrm{H}_{4}$ \\
\hline-1345.5717 & -1345.1293 & 2.14 & 0.027 & -6.802 & -8.032 & 0.323 & 0.091 & ${ }^{5} \mathrm{H}_{4}$ \\
\hline-1345.5735 & -1345.1315 & 0.76 & 0.275 & -6.563 & -8.159 & 0.163 & 0.164 & ${ }^{4} \mathrm{H}_{5}$ \\
\hline-1345.5754 & -1345.1311 & 1.00 & 0.184 & -6.675 & -8.011 & 0.305 & 0.098 & ${ }^{5} \mathrm{H}_{4}$ \\
\hline-1345.5750 & -1345.1310 & 1.08 & 0.161 & -6.659 & -8.143 & 0.184 & 0.163 & ${ }^{4} \mathrm{H}_{5}$ \\
\hline-1345.5725 & -1345.1295 & 2.03 & 0.033 & -6.669 & -8.185 & 0.187 & 0.160 & ${ }^{4} \mathrm{H}_{5}$ \\
\hline-1345.5729 & -1345.1291 & 2.30 & 0.021 & -6.715 & -8.007 & 0.306 & 0.095 & ${ }^{5} \mathrm{H}_{4}$ \\
\hline-1345.5738 & -1345.1295 & 2.02 & 0.033 & -6.712 & -8.012 & 0.336 & 0.084 & ${ }^{5} \mathrm{H}_{4}$ \\
\hline-1345.5760 & -1345.1313 & 0.88 & 0.226 & -6.810 & -8.088 & 0.301 & 0.109 & ${ }^{5} \mathrm{H}_{4}$ \\
\hline-1345.5765 & -1345.1325 & 0.14 & 0.789 & -6.813 & -8.059 & 0.307 & 0.095 & ${ }^{5} \mathrm{H}_{4}$ \\
\hline-1345.5731 & -1345.1306 & 1.32 & 0.108 & -6.626 & -8.143 & 0.175 & 0.163 & ${ }^{4} \mathrm{H}_{5}$ \\
\hline-1345.5708 & -1345.1286 & 2.60 & 0.013 & -6.700 & -8.057 & 0.319 & 0.095 & ${ }^{5} \mathrm{H}_{4}$ \\
\hline-1345.5742 & -1345.1304 & 1.47 & 0.084 & -6.784 & -8.117 & 0.281 & 0.120 & ${ }^{5} \mathrm{H}_{4}$ \\
\hline-1345.5743 & -1345.1303 & 1.49 & 0.081 & -6.574 & -8.147 & 0.141 & 0.172 & ${ }^{4} \mathrm{H}_{5}$ \\
\hline-1345.5751 & -1345.1305 & 1.42 & 0.091 & -6.754 & -8.024 & 0.317 & 0.092 & ${ }^{5} \mathrm{H}_{4}$ \\
\hline-1345.5766 & -1345.1327 & 0.00 & 1.000 & -6.812 & -8.054 & 0.308 & 0.094 & ${ }^{5} \mathbf{H}_{4}$ \\
\hline-1345.5735 & -1345.1292 & 2.24 & 0.023 & -6.713 & -8.013 & 0.338 & 0.083 & ${ }^{5} \mathrm{H}_{4}$ \\
\hline-1345.5763 & -1345.1324 & 0.22 & 0.684 & -6.709 & -8.012 & 0.312 & 0.096 & ${ }^{5} \mathrm{H}_{4}$ \\
\hline-1345.5752 & -1345.1319 & 0.49 & 0.434 & -6.591 & -8.146 & 0.171 & 0.163 & ${ }^{4} \mathrm{H}_{5}$ \\
\hline
\end{tabular}

Table SCY3. Energetics for 3 at the PBE0/D3BJ/CPCM $\left(\mathrm{CH}_{3} \mathrm{CN}\right) / / \mathrm{PBE} 0 / \mathrm{D} 3 \mathrm{BJ} / \mathrm{DEF} 2-$ $\mathrm{SVP} / \mathrm{RIJCOSX}$ level. Relative energies in $\mathrm{kcal} / \mathrm{mol}$, orbital energies in $\mathrm{eV}$.

\begin{tabular}{|c|c|c|c|c|c|c|c|c|}
\hline E (a.u.) & G (a.u.) & $\Delta \mathrm{G}$ & Boltz. & HOMO & $\begin{array}{c}\pi_{C_{1}-C_{2}} \\
\quad(\mathrm{ev})\end{array}$ & $\begin{array}{c}\Delta E^{(2)}(e V) \\
\left(\pi \rightarrow \sigma_{C_{3}-o}^{*}\right)\end{array}$ & $\begin{array}{c}\Delta E^{(2)}(e V) \\
\left(\pi \rightarrow \sigma_{C_{3}-H}^{*}\right)\end{array}$ & Conforme \\
\hline-652.9502 & -652.7399 & 0.84 & 0.242 & -6.781 & -8.016 & 0.335 & 0.085 & ${ }^{5} \mathrm{H}_{4}$ \\
\hline-652.9493 & -652.7388 & 1.55 & 0.073 & -6.806 & -8.024 & 0.340 & 0.082 & ${ }^{5} \mathrm{H}_{4}$ \\
\hline-652.9487 & -652.7383 & 1.86 & 0.043 & -6.792 & -8.026 & 0.335 & 0.085 & ${ }^{5} \mathrm{H}_{4}$ \\
\hline-652.9481 & -652.7376 & 2.27 & 0.022 & -6.663 & -8.011 & 0.334 & 0.084 & ${ }^{5} \mathrm{H}_{4}$ \\
\hline-652.9500 & -652.7395 & 1.12 & 0.151 & -6.771 & -8.006 & 0.305 & 0.091 & ${ }^{5} \mathrm{H}_{4}$ \\
\hline-652.9506 & -652.7405 & 0.51 & 0.425 & -6.766 & -8.011 & 0.301 & 0.098 & ${ }^{5} \mathbf{H}_{4}$ \\
\hline-652.9498 & -652.7392 & 1.30 & 0.112 & -6.785 & -8.010 & 0.299 & 0.095 & ${ }^{5} \mathrm{H}_{4}$ \\
\hline-652.9499 & -652.7396 & 1.05 & 0.170 & -6.677 & -8.139 & 0.176 & 0.163 & ${ }^{4} \mathrm{H}_{5}$ \\
\hline-652.9486 & -652.7382 & 1.90 & 0.040 & -6.648 & -8.009 & 0.299 & 0.097 & ${ }^{5} \mathrm{H}_{4}$ \\
\hline-652.9458 & -652.7349 & 4.01 & 0.001 & -6.739 & -8.042 & 0.288 & 0.110 & ${ }^{5} \mathrm{H}_{4}$ \\
\hline-652.9512 & -652.7413 & 0.00 & 1.000 & -6.650 & -8.107 & 0.176 & 0.166 & ${ }^{4} \mathrm{H}_{5}$ \\
\hline-652.9499 & -652.7407 & 0.38 & 0.529 & -6.698 & -8.115 & 0.182 & 0.167 & ${ }^{4} \mathrm{H}_{5}$ \\
\hline-652.9497 & -652.7394 & 1.15 & 0.143 & -6.661 & -8.110 & 0.180 & 0.164 & ${ }^{4} \mathrm{H}_{5}$ \\
\hline-652.9485 & -652.7386 & 1.70 & 0.057 & -6.562 & -8.123 & 0.152 & 0.166 & ${ }^{4} \mathrm{H}_{5}$ \\
\hline-652.9489 & -652.7398 & 0.93 & 0.207 & -6.695 & -8.106 & 0.182 & 0.167 & ${ }^{4} \mathrm{H}_{5}$ \\
\hline-652.9477 & -652.7383 & 1.86 & 0.044 & -6.689 & -8.115 & 0.181 & 0.166 & ${ }^{4} \mathrm{H}_{5}$ \\
\hline
\end{tabular}


Table SCY4. Energetics for 4 at the PBE0/D3BJ/CPCM $\left(\mathrm{CH}_{3} \mathrm{CN}\right) / / \mathrm{PBE} 0 / \mathrm{D} 3 \mathrm{BJ} / \mathrm{DEF} 2-$ $\mathrm{SVP} / \mathrm{RIJCOSX}$ level. Relative energies in $\mathrm{kcal} / \mathrm{mol}$, orbital energies in $\mathrm{eV}$.

\begin{tabular}{|c|c|c|c|c|c|c|c|c|}
\hline E (a.u.) & G (a.u.) & $\Delta \mathrm{G}$ & Boltz. & HOMO & $\begin{array}{c}\pi_{C_{1}-C_{2}} \\
(\mathrm{ev})\end{array}$ & $\begin{array}{c}\Delta E^{(2)}(e V) \\
\left(\pi \rightarrow \sigma_{C_{3}-O}^{*}\right)\end{array}$ & $\begin{array}{c}\Delta E^{(2)}(e V) \\
\left(\pi \rightarrow \sigma_{C_{3}-H}^{*}\right)\end{array}$ & Conformer \\
\hline-770.8052 & -770.5140 & 2.89 & 0.008 & -6.763 & -8.009 & 0.329 & 0.086 & ${ }^{5} \mathrm{H}_{4}$ \\
\hline-770.8076 & -770.5167 & 1.15 & 0.144 & -6.549 & -8.096 & 0.167 & 0.163 & ${ }^{4} \mathrm{H}_{5}$ \\
\hline-770.8064 & -770.5148 & 2.37 & 0.018 & -6.722 & -8.005 & 0.308 & 0.090 & ${ }^{5} \mathrm{H}_{4}$ \\
\hline-770.8063 & -770.5160 & 1.64 & 0.063 & -6.619 & -8.113 & 0.172 & 0.166 & ${ }^{4} \mathrm{H}_{5}$ \\
\hline-770.8046 & -770.5137 & 3.05 & 0.006 & -6.746 & -7.990 & 0.293 & 0.097 & ${ }^{5} \mathrm{H}_{4}$ \\
\hline-770.8062 & -770.5156 & 1.84 & 0.045 & -6.681 & -8.090 & 0.183 & 0.166 & ${ }^{4} \mathrm{H}_{5}$ \\
\hline-770.8051 & -770.5137 & 3.05 & 0.006 & -6.720 & -7.992 & 0.303 & 0.096 & ${ }^{5} \mathrm{H}_{4}$ \\
\hline-770.8070 & -770.5167 & 1.16 & 0.141 & -6.623 & -8.097 & 0.172 & 0.167 & ${ }^{4} \mathrm{H}_{5}$ \\
\hline-770.8063 & -770.5154 & 1.99 & 0.035 & -6.777 & -8.012 & 0.330 & 0.085 & ${ }^{5} \mathrm{H}_{4}$ \\
\hline-770.8069 & -770.5159 & 1.68 & 0.059 & -6.790 & -8.011 & 0.334 & 0.082 & ${ }^{5} \mathrm{H}_{4}$ \\
\hline-770.8051 & -770.5151 & 2.18 & 0.025 & -6.656 & -8.111 & 0.175 & 0.167 & ${ }^{4} \mathrm{H}_{5}$ \\
\hline-770.8055 & -770.5158 & 1.76 & 0.051 & -6.663 & -8.107 & 0.176 & 0.168 & ${ }^{4} \mathrm{H}_{5}$ \\
\hline-770.8055 & -770.5161 & 1.52 & 0.076 & -6.681 & -8.092 & 0.184 & 0.166 & ${ }^{4} \mathrm{H}_{5}$ \\
\hline-770.8064 & -770.5156 & 1.88 & 0.042 & -6.517 & -8.121 & 0.151 & 0.165 & ${ }^{4} \mathrm{H}_{5}$ \\
\hline-770.8079 & -770.5165 & 1.29 & 0.114 & -6.767 & -7.997 & 0.295 & 0.095 & ${ }^{5} \mathrm{H}_{4}$ \\
\hline-770.8059 & -770.5146 & 2.49 & 0.015 & -6.747 & -7.993 & 0.294 & 0.096 & ${ }^{5} \mathrm{H}_{4}$ \\
\hline-770.8063 & -770.5152 & 2.08 & 0.030 & -6.747 & -8.010 & 0.300 & 0.095 & ${ }^{5} \mathrm{H}_{4}$ \\
\hline-770.8040 & -770.5123 & 3.90 & 0.001 & -6.706 & -8.035 & 0.284 & 0.109 & ${ }^{4} \mathrm{H}_{5}$ \\
\hline-770.8076 & -770.5168 & 1.09 & 0.159 & -6.655 & -8.133 & 0.169 & 0.164 & ${ }^{4} \mathrm{H}_{5}$ \\
\hline-770.8071 & -770.5153 & 2.03 & 0.033 & -6.769 & -7.993 & 0.297 & 0.095 & ${ }^{5} \mathrm{H}_{4}$ \\
\hline-770.8070 & -770.5185 & 0.05 & 0.921 & -6.687 & -8.095 & 0.185 & 0.166 & ${ }^{4} \mathrm{H}_{5}$ \\
\hline-770.8060 & -770.5146 & 2.49 & 0.015 & -6.738 & -7.999 & 0.297 & 0.093 & ${ }^{5} \mathrm{H}_{4}$ \\
\hline-770.8075 & -770.5159 & 1.67 & 0.059 & -6.758 & -7.997 & 0.300 & 0.094 & ${ }^{5} \mathrm{H}_{4}$ \\
\hline-770.8084 & -770.5169 & 1.01 & 0.181 & -6.753 & -8.003 & 0.299 & 0.093 & ${ }^{5} \mathbf{H}_{4}$ \\
\hline-770.8076 & -770.5170 & 1.01 & 0.183 & -6.621 & -8.090 & 0.173 & 0.166 & ${ }^{4} \mathrm{H}_{5}$ \\
\hline-770.8071 & -770.5165 & 1.29 & 0.113 & -6.486 & -8.118 & 0.156 & 0.166 & ${ }^{4} \mathrm{H}_{5}$ \\
\hline-770.8079 & -770.5168 & 1.11 & 0.153 & -6.621 & -8.091 & 0.170 & 0.168 & ${ }^{4} \mathrm{H}_{5}$ \\
\hline-770.8083 & -770.5179 & 0.41 & 0.504 & -6.497 & -8.114 & 0.149 & 0.166 & ${ }^{4} \mathrm{H}_{5}$ \\
\hline-770.8065 & -770.5161 & 1.57 & 0.071 & -6.670 & -8.104 & 0.177 & 0.168 & ${ }^{4} \mathrm{H}_{5}$ \\
\hline-770.8073 & -770.5165 & 1.29 & 0.114 & -6.639 & -8.103 & 0.176 & 0.164 & ${ }^{4} \mathrm{H}_{5}$ \\
\hline-770.8053 & -770.5140 & 2.85 & 0.008 & -6.772 & -8.002 & 0.332 & 0.084 & ${ }^{5} \mathrm{H}_{4}$ \\
\hline-770.8058 & -770.5148 & 2.35 & 0.019 & -6.747 & -8.001 & 0.304 & 0.093 & ${ }^{5} \mathrm{H}_{4}$ \\
\hline-770.8089 & -770.5186 & 0.00 & 1.000 & -6.626 & -8.098 & 0.173 & 0.166 & ${ }^{4} \mathrm{H}_{5}$ \\
\hline
\end{tabular}

Table SCY5. Energetics for 5 at the PBE0/D3BJ/CPCM $\left(\mathrm{CH}_{3} \mathrm{CN}\right) / / \mathrm{PBE} 0 / \mathrm{D} 3 \mathrm{BJ} / \mathrm{DEF} 2-$ $\mathrm{SVP} / \mathrm{RIJCOSX}$ level. Relative energies in $\mathrm{kcal} / \mathrm{mol}$, orbital energies in $\mathrm{eV}$.

\begin{tabular}{|c|c|c|c|c|c|c|c|c|}
\hline E (a.u.) & G (a.u.) & $\Delta \mathrm{G}$ & Boltz. & HOMO & $\begin{array}{c}\pi_{C_{1}-C_{2}} \\
(\mathrm{ev})\end{array}$ & $\begin{array}{c}\Delta E^{(2)}(e V) \\
\left(\pi \rightarrow \sigma_{C_{3}-o}^{*}\right)\end{array}$ & $\begin{array}{c}\Delta E^{(2)}(e V) \\
\left(\pi \rightarrow \sigma_{C_{3}-H}^{*}\right)\end{array}$ & Conformer \\
\hline-2114.1820 & -2113.5224 & 1.17 & 0.138 & -6.652 & -7.930 & 0.318 & 0.082 & ${ }^{5} \mathrm{H}_{4}$ \\
\hline-2114.1800 & -2113.5211 & 2.02 & 0.033 & -6.660 & -7.962 & 0.309 & 0.090 & ${ }^{5} \mathrm{H}_{4}$ \\
\hline-2114.1821 & -2113.5211 & 2.04 & 0.032 & -6.655 & -7.984 & 0.315 & 0.096 & ${ }^{5} \mathrm{H}_{4}$ \\
\hline-2114.1804 & -2113.5201 & 2.64 & 0.012 & -6.635 & -7.932 & 0.323 & 0.084 & ${ }^{5} \mathrm{H}_{4}$ \\
\hline-2114.1810 & -2113.5222 & 1.30 & 0.111 & -6.666 & -7.979 & 0.310 & 0.095 & ${ }^{5} \mathrm{H}_{4}$ \\
\hline-2114.1815 & -2113.5216 & 1.72 & 0.055 & -6.650 & -7.963 & 0.318 & 0.092 & ${ }^{5} \mathrm{H}_{4}$ \\
\hline-2114.1812 & -2113.5223 & 1.25 & 0.122 & -6.655 & -7.944 & 0.323 & 0.086 & ${ }^{5} \mathrm{H}_{4}$ \\
\hline-2114.1830 & -2113.5230 & 0.81 & 0.256 & -6.654 & -7.978 & 0.307 & 0.093 & ${ }^{5} \mathrm{H}_{4}$ \\
\hline-2114.1825 & -2113.5227 & 1.02 & 0.179 & -6.646 & -7.972 & 0.305 & 0.094 & ${ }^{5} \mathrm{H}_{4}$ \\
\hline-2114.1823 & -2113.5222 & 1.31 & 0.109 & -6.635 & -7.954 & 0.303 & 0.089 & ${ }^{5} \mathrm{H}_{4}$ \\
\hline-2114.1812 & -2113.5202 & 2.59 & 0.013 & -6.657 & -7.951 & 0.307 & 0.089 & ${ }^{5} \mathrm{H}_{4}$ \\
\hline-2114.1808 & -2113.5220 & 1.48 & 0.082 & -6.636 & -7.954 & 0.309 & 0.091 & ${ }^{5} \mathrm{H}_{4}$ \\
\hline-2114.1815 & -2113.5216 & 1.69 & 0.057 & -6.633 & -7.946 & 0.309 & 0.086 & ${ }^{5} \mathrm{H}_{4}$ \\
\hline-2114.1818 & -2113.5211 & 2.00 & 0.034 & -6.631 & -7.935 & 0.312 & 0.090 & ${ }^{5} \mathrm{H}_{4}$ \\
\hline-2114.1810 & -2113.5199 & 2.76 & 0.010 & -6.644 & -7.972 & 0.314 & 0.095 & ${ }^{5} \mathrm{H}_{4}$ \\
\hline-2114.1805 & -2113.5197 & 2.92 & 0.007 & -6.653 & -7.997 & 0.313 & 0.099 & ${ }^{5} \mathrm{H}_{4}$ \\
\hline-2114.1826 & -2113.5218 & 1.58 & 0.070 & -6.645 & -7.948 & 0.309 & 0.088 & ${ }^{5} \mathrm{H}_{4}$ \\
\hline
\end{tabular}




\begin{tabular}{|c|c|c|c|c|c|c|c|}
\hline-2114.1803 & -2113.5216 & 1.71 & 0.056 & -6.638 & -7.945 & 0.320 & 0.087 \\
\hline-2114.1816 & -2113.5225 & 1.12 & 0.151 & -6.657 & -7.954 & 0.316 & 0.093 \\
\hline-2114.1815 & -2113.5199 & 2.74 & 0.010 & -6.627 & -7.950 & 0.320 & 0.091 \\
\hline-2114.1815 & -2113.5199 & 2.74 & 0.010 & -6.627 & -7.950 & 0.320 & 0.091 \\
\hline-2114.1809 & -2113.5200 & 2.74 & 0.010 & -6.647 & -7.958 & 0.309 & 0.092 \\
\hline-2114.1800 & -2113.5188 & 3.44 & 0.003 & -6.662 & -8.027 & 0.297 & 0.103 \\
\hline-2114.1816 & -2113.5220 & 1.44 & 0.088 & -6.646 & -7.954 & 0.318 & 0.090 \\
\hline-2114.1804 & -2113.5218 & 1.60 & 0.067 & -6.642 & -7.923 & 0.307 & 0.083 \\
\hline-2114.1816 & -2113.5213 & 1.86 & 0.043 & -6.645 & -7.948 & 0.317 & 0.091 \\
\hline-2114.1812 & -2113.5221 & 1.37 & 0.099 & -6.657 & -7.968 & 0.313 & 0.096 \\
\hline-2114.1815 & -2113.5220 & 1.48 & 0.082 & -6.651 & -7.950 & 0.312 & 0.088 \\
\hline-2114.1820 & -2113.5228 & 0.98 & 0.191 & -6.653 & -7.957 & 0.316 & 0.091 \\
\hline-2114.1816 & -2113.5208 & 2.18 & 0.025 & -6.655 & -7.988 & 0.316 & 0.095 \\
\hline-2114.1809 & -2113.5202 & 2.62 & 0.012 & -6.642 & -7.937 & 0.313 & 0.087 \\
\hline-2114.1816 & -2113.5221 & 1.42 & 0.091 & -6.650 & -7.937 & 0.318 & 0.084 \\
\hline-2114.1810 & -2113.5213 & 1.92 & 0.039 & -6.644 & -7.937 & 0.317 & 0.083 \\
\hline-2114.1785 & -2113.5195 & 3.00 & 0.006 & -6.638 & -7.914 & 0.302 & 0.083 \\
\hline-2114.1812 & -2113.5215 & 1.78 & 0.050 & -6.647 & -7.939 & 0.308 & 0.087 \\
\hline-2114.1728 & -2113.5129 & 7.19 & 0.000 & -6.617 & -7.947 & 0.315 & 0.090 \\
\hline-2114.1801 & -2113.5204 & 2.45 & 0.016 & -6.640 & -7.932 & 0.326 & 0.083 \\
\hline-2114.1812 & -2113.5218 & 1.55 & 0.073 & -6.644 & -7.939 & 0.305 & 0.086 \\
\hline-2114.1808 & -2113.5214 & 1.83 & 0.045 & -6.659 & -7.984 & 0.309 & 0.098 \\
\hline-2114.1811 & -2113.5212 & 1.97 & 0.036 & -6.630 & -7.936 & 0.314 & 0.083 \\
\hline-2114.1817 & -2113.5222 & 1.33 & 0.106 & -6.649 & -7.954 & 0.318 & 0.090 \\
\hline-2114.1826 & -2113.5234 & 0.58 & 0.377 & -6.648 & -7.952 & 0.310 & 0.088 \\
\hline-2114.1811 & -2113.5221 & 1.40 & 0.094 & -6.652 & -7.955 & 0.317 & 0.093 \\
\hline-2114.1814 & -2113.5210 & 2.09 & 0.030 & -6.650 & -7.932 & 0.316 & 0.085 \\
\hline-2114.1819 & -2113.5224 & 1.19 & 0.134 & -6.652 & -7.962 & 0.308 & 0.092 \\
\hline-2114.1801 & -2113.5207 & 2.28 & 0.021 & -6.653 & -7.962 & 0.314 & 0.091 \\
\hline-2114.1815 & -2113.5218 & 1.58 & 0.069 & -6.658 & -7.966 & 0.309 & 0.091 \\
\hline-2114.1814 & -2113.5203 & 2.51 & 0.014 & -6.645 & -7.963 & 0.308 & 0.092 \\
\hline-2114.1821 & -2113.5228 & 0.95 & 0.203 & -6.656 & -7.964 & 0.309 & 0.091 \\
\hline-2114.1824 & -2113.5217 & 1.64 & 0.062 & -6.639 & -7.968 & 0.320 & 0.092 \\
\hline-2114.1826 & -2113.5224 & 1.21 & 0.131 & -6.646 & -7.948 & 0.313 & 0.085 \\
\hline-2114.1820 & -2113.5222 & 1.34 & 0.105 & -6.648 & -7.943 & 0.314 & 0.087 \\
\hline-2114.1829 & -2113.5219 & 1.54 & 0.074 & -6.646 & -7.970 & 0.320 & 0.092 \\
\hline-2114.1808 & -2113.5217 & 1.63 & 0.064 & -6.638 & -7.917 & 0.310 & 0.079 \\
\hline-2114.1815 & -2113.5218 & 1.58 & 0.070 & -6.654 & -7.967 & 0.314 & 0.094 \\
\hline-2114.1825 & -2113.5230 & 0.80 & 0.259 & -6.631 & -7.924 & 0.312 & 0.082 \\
\hline-2114.1812 & -2113.5205 & 2.40 & 0.017 & -6.647 & -7.944 & 0.312 & 0.089 \\
\hline-2114.1807 & -2113.5213 & 1.92 & 0.039 & -6.649 & -7.943 & 0.310 & 0.087 \\
\hline-2114.1820 & -2113.5211 & 2.04 & 0.032 & -6.642 & -7.948 & 0.307 & 0.088 \\
\hline-2114.1811 & -2113.5214 & 1.86 & 0.043 & -6.641 & -7.940 & 0.325 & 0.085 \\
\hline-2114.1799 & -2113.5205 & 2.39 & 0.018 & -6.634 & -7.929 & 0.307 & 0.083 \\
\hline-2114.1820 & -2113.5228 & 0.97 & 0.193 & -6.656 & -7.961 & 0.310 & 0.090 \\
\hline-2114.1801 & -2113.5204 & 2.44 & 0.016 & -6.647 & -7.948 & 0.319 & 0.090 \\
\hline-2114.1815 & -2113.5204 & 2.49 & 0.015 & -6.647 & -7.962 & 0.309 & 0.092 \\
\hline-2114.1813 & -2113.5213 & 1.89 & 0.041 & -6.649 & -7.961 & 0.312 & 0.091 \\
\hline-2114.1818 & -2113.5219 & 1.54 & 0.074 & -6.632 & -7.945 & 0.308 & 0.088 \\
\hline-2114.1833 & -2113.5243 & 0.00 & 1.000 & -6.660 & -7.966 & 0.309 & 0.088 \\
\hline-2114.1818 & -2113.5223 & 1.24 & 0.123 & -6.654 & -7.965 & 0.308 & 0.091 \\
\hline-2114.1818 & -2113.5223 & 1.24 & 0.123 & -6.654 & -7.965 & 0.308 & 0.091 \\
\hline-2114.1818 & -2113.5212 & 1.96 & 0.037 & -6.653 & -7.986 & 0.315 & 0.096 \\
\hline-2114.1821 & -2113.5214 & 1.84 & 0.045 & -6.646 & -7.951 & 0.308 & 0.088 \\
\hline-2114.1810 & -2113.5214 & 1.86 & 0.043 & -6.653 & -7.965 & 0.318 & 0.093 \\
\hline-2114.1823 & -2113.5229 & 0.88 & 0.227 & -6.659 & -7.968 & 0.314 & 0.093 \\
\hline-2114.1805 & -2113.5209 & 2.14 & 0.027 & -6.649 & -7.952 & 0.317 & 0.091 \\
\hline-2114.1814 & -2113.5219 & 1.50 & 0.079 & -6.650 & -7.935 & 0.312 & 0.085 \\
\hline-2114.1809 & -2113.5211 & 2.03 & 0.032 & -6.659 & -7.979 & 0.313 & 0.097 \\
\hline-2114.1807 & -2113.5209 & 2.16 & 0.026 & -6.654 & -7.978 & 0.315 & 0.097 \\
\hline-2114.1818 & -2113.5225 & 1.16 & 0.142 & -6.638 & -7.953 & 0.308 & 0.089 \\
\hline-2114.1801 & -2113.5209 & 2.15 & 0.027 & -6.634 & -7.929 & 0.307 & 0.083 \\
\hline
\end{tabular}


Table SCY6. Energetics for 6 at the PBE0/D3BJ/CPCM $\left(\mathrm{CH}_{3} \mathrm{CN}\right) / / \mathrm{PBE} / \mathrm{D} 3 \mathrm{BJ} / \mathrm{DEF} 2-$ $\mathrm{SVP} / \mathrm{RIJCOSX}$ level. Relative energies in $\mathrm{kcal} / \mathrm{mol}$, orbital energies in $\mathrm{eV}$.

\begin{tabular}{|c|c|c|c|c|c|c|c|c|}
\hline E (a.u.) & G (a.u.) & $\Delta \mathrm{G}$ & Boltz. & $\begin{array}{c}\text { HOMO } \\
-3\end{array}$ & $\begin{array}{c}\pi_{C_{1}-C_{2}} \\
(\mathrm{ev})\end{array}$ & $\begin{array}{c}\Delta E^{(2)}(e V) \\
\left(\pi \rightarrow \sigma_{C_{3}-O}^{*}\right)\end{array}$ & $\begin{array}{c}\Delta E^{(2)}(e V) \\
\left(\pi \rightarrow \sigma_{C_{3}-H}^{*}\right)\end{array}$ & Conformer \\
\hline-1688.9299 & -1688.3935 & 0.53 & 0.406 & -6.794 & -8.081 & 0.299 & 0.110 & ${ }^{5} \mathrm{H}_{4}$ \\
\hline-1688.9279 & -1688.3921 & 1.39 & 0.095 & -6.785 & -8.061 & 0.304 & 0.104 & ${ }^{5} \mathrm{H}_{4}$ \\
\hline-1688.9278 & -1688.3913 & 1.91 & 0.040 & -6.821 & -7.990 & 0.331 & 0.085 & ${ }^{5} \mathrm{H}_{4}$ \\
\hline-1688.9296 & -1688.3933 & 0.66 & 0.329 & -6.791 & -8.070 & 0.304 & 0.102 & ${ }^{5} \mathrm{H}_{4}$ \\
\hline-1688.9281 & -1688.3910 & 2.12 & 0.028 & -6.757 & -7.993 & 0.306 & 0.096 & ${ }^{5} \mathrm{H}_{4}$ \\
\hline-1688.9301 & -1688.3925 & 1.16 & 0.141 & -6.716 & -7.975 & 0.310 & 0.091 & ${ }^{5} \mathrm{H}_{4}$ \\
\hline-1688.9282 & -1688.3910 & 2.09 & 0.029 & -6.761 & -7.998 & 0.305 & 0.097 & ${ }^{5} \mathrm{H}_{4}$ \\
\hline-1688.9307 & -1688.3943 & 0.00 & 1.000 & -6.795 & -8.070 & 0.312 & 0.097 & ${ }^{5} \mathbf{H}_{4}$ \\
\hline-1688.9245 & -1688.3897 & 2.88 & 0.008 & -6.793 & -7.956 & 0.316 & 0.089 & ${ }^{5} \mathrm{H}_{4}$ \\
\hline-1688.9294 & -1688.3928 & 0.94 & 0.204 & -6.774 & -7.988 & 0.344 & 0.080 & ${ }^{5} \mathrm{H}_{4}$ \\
\hline-1688.9299 & -1688.3932 & 0.71 & 0.300 & -6.793 & -7.985 & 0.294 & 0.103 & ${ }^{5} \mathrm{H}_{4}$ \\
\hline-1688.9297 & -1688.3930 & 0.86 & 0.233 & -6.732 & -7.987 & 0.307 & 0.094 & ${ }^{5} \mathrm{H}_{4}$ \\
\hline-1688.9288 & -1688.3930 & 0.81 & 0.255 & -6.777 & -8.084 & 0.298 & 0.110 & ${ }^{5} \mathrm{H}_{4}$ \\
\hline-1688.9274 & -1688.3913 & 1.93 & 0.038 & -6.801 & -7.997 & 0.315 & 0.090 & ${ }^{5} \mathrm{H}_{4}$ \\
\hline-1688.9295 & -1688.3929 & 0.92 & 0.212 & -6.812 & -8.007 & 0.297 & 0.098 & ${ }^{5} \mathrm{H}_{4}$ \\
\hline-1688.9288 & -1688.3928 & 0.96 & 0.197 & -6.658 & -8.093 & 0.164 & 0.162 & ${ }^{4} \mathrm{H}_{5}$ \\
\hline-1688.9300 & -1688.3936 & 0.47 & 0.452 & -6.793 & -8.079 & 0.299 & 0.110 & ${ }^{5} \mathrm{H}_{4}$ \\
\hline-1688.9283 & -1688.3924 & 1.22 & 0.127 & -6.788 & -8.062 & 0.307 & 0.102 & ${ }^{5} \mathrm{H}_{4}$ \\
\hline-1688.9275 & -1688.3904 & 2.46 & 0.016 & -6.820 & -7.990 & 0.306 & 0.097 & ${ }^{5} \mathrm{H}_{4}$ \\
\hline-1688.9285 & -1688.3931 & 0.77 & 0.272 & -6.805 & -8.062 & 0.310 & 0.097 & ${ }^{5} \mathrm{H}_{4}$ \\
\hline-1688.9276 & -1688.3917 & 1.68 & 0.059 & -6.785 & -7.974 & 0.309 & 0.094 & ${ }^{5} \mathrm{H}_{4}$ \\
\hline-1688.9291 & -1688.3932 & 0.72 & 0.295 & -6.752 & -7.980 & 0.311 & 0.094 & ${ }^{5} \mathrm{H}_{4}$ \\
\hline-1688.9263 & -1688.3893 & 3.13 & 0.005 & -6.751 & -7.934 & 0.341 & 0.069 & ${ }^{5} \mathrm{H}_{4}$ \\
\hline-1688.9300 & -1688.3929 & 0.87 & 0.231 & -6.714 & -7.978 & 0.310 & 0.092 & ${ }^{5} \mathrm{H}_{4}$ \\
\hline-1688.9284 & -1688.3912 & 1.95 & 0.037 & -6.760 & -7.996 & 0.304 & 0.098 & ${ }^{5} \mathrm{H}_{4}$ \\
\hline-1688.9248 & -1688.3880 & 3.97 & 0.001 & -6.800 & -7.949 & 0.343 & 0.069 & ${ }^{5} \mathrm{H}_{4}$ \\
\hline-1688.9302 & -1688.3925 & 1.12 & 0.151 & -6.716 & -7.975 & 0.310 & 0.091 & ${ }^{5} \mathrm{H}_{4}$ \\
\hline-1688.9293 & -1688.3920 & 1.47 & 0.083 & -6.734 & -7.991 & 0.315 & 0.092 & ${ }^{5} \mathrm{H}_{4}$ \\
\hline-1688.9287 & -1688.3936 & 0.49 & 0.440 & -6.805 & -8.041 & 0.304 & 0.098 & ${ }^{5} \mathrm{H}_{4}$ \\
\hline-1688.9273 & -1688.3915 & 1.79 & 0.049 & -6.807 & -8.006 & 0.313 & 0.091 & ${ }^{5} \mathrm{H}_{4}$ \\
\hline-1688.9304 & -1688.3932 & 0.70 & 0.306 & -6.805 & -7.989 & 0.297 & 0.102 & ${ }^{5} \mathrm{H}_{4}$ \\
\hline-1688.9304 & -1688.3933 & 0.67 & 0.320 & -6.846 & -7.959 & 0.306 & 0.095 & ${ }^{5} \mathrm{H}_{4}$ \\
\hline-1688.9298 & -1688.3934 & 0.60 & 0.363 & -6.792 & -8.084 & 0.298 & 0.111 & ${ }^{5} \mathrm{H}_{4}$ \\
\hline-1688.9282 & -1688.3926 & 1.09 & 0.159 & -6.769 & -7.977 & 0.307 & 0.095 & ${ }^{5} \mathrm{H}_{4}$ \\
\hline-1688.9259 & -1688.3903 & 2.53 & 0.014 & -6.795 & -8.030 & 0.335 & 0.097 & ${ }^{5} \mathrm{H}_{4}$ \\
\hline-1688.9268 & -1688.3908 & 2.23 & 0.023 & -6.794 & -8.002 & 0.328 & 0.098 & ${ }^{5} \mathrm{H}_{4}$ \\
\hline-1688.9258 & -1688.3905 & 2.41 & 0.017 & -6.765 & -7.976 & 0.343 & 0.080 & ${ }^{5} \mathrm{H}_{4}$ \\
\hline-1688.9289 & -1688.3929 & 0.90 & 0.219 & -6.754 & -7.983 & 0.312 & 0.093 & ${ }^{5} \mathrm{H}_{4}$ \\
\hline-1688.9298 & -1688.3936 & 0.49 & 0.437 & -6.785 & -8.102 & 0.299 & 0.110 & ${ }^{5} \mathrm{H}_{4}$ \\
\hline-1688.9304 & -1688.3940 & 0.23 & 0.679 & -6.764 & -7.990 & 0.308 & 0.097 & ${ }^{5} \mathrm{H}_{4}$ \\
\hline-1688.9301 & -1688.3941 & 0.15 & 0.776 & -6.766 & -7.991 & 0.307 & 0.097 & ${ }^{5} \mathrm{H}_{4}$ \\
\hline-1688.9271 & -1688.3911 & 2.05 & 0.031 & -6.810 & -8.007 & 0.316 & 0.090 & ${ }^{5} \mathrm{H}_{4}$ \\
\hline-1688.9244 & -1688.3898 & 2.82 & 0.009 & -6.771 & -7.944 & 0.317 & 0.088 & ${ }^{5} \mathrm{H}_{4}$ \\
\hline-1688.9286 & -1688.3919 & 1.55 & 0.074 & -6.763 & -7.971 & 0.310 & 0.094 & ${ }^{5} \mathrm{H}_{4}$ \\
\hline-1688.9272 & -1688.3915 & 1.79 & 0.049 & -6.795 & -8.070 & 0.298 & 0.109 & ${ }^{5} \mathrm{H}_{4}$ \\
\hline-1688.9253 & -1688.3894 & 3.11 & 0.005 & -6.725 & -8.132 & 0.199 & 0.159 & ${ }^{4} \mathrm{H}_{5}$ \\
\hline-1688.9283 & -1688.3923 & 1.25 & 0.122 & -6.685 & -8.129 & 0.180 & 0.163 & ${ }^{4} \mathrm{H}_{5}$ \\
\hline-1688.9276 & -1688.3916 & 1.72 & 0.055 & -6.610 & -8.113 & 0.159 & 0.162 & ${ }^{4} \mathrm{H}_{5}$ \\
\hline-1688.9300 & -1688.3935 & 0.50 & 0.431 & -6.793 & -8.078 & 0.299 & 0.110 & ${ }^{5} \mathrm{H}_{4}$ \\
\hline-1688.9272 & -1688.3914 & 1.86 & 0.043 & -6.743 & -7.979 & 0.313 & 0.095 & ${ }^{5} \mathrm{H}_{4}$ \\
\hline-1688.9261 & -1688.3886 & 3.58 & 0.002 & -6.769 & -8.061 & 0.328 & 0.101 & ${ }^{5} \mathrm{H}_{4}$ \\
\hline-1688.9304 & -1688.3939 & 0.29 & 0.608 & -6.769 & -7.991 & 0.308 & 0.096 & ${ }^{5} \mathrm{H}_{4}$ \\
\hline-1688.9297 & -1688.3928 & 0.99 & 0.187 & -6.820 & -8.005 & 0.298 & 0.098 & ${ }^{5} \mathrm{H}_{4}$ \\
\hline-1688.9263 & -1688.3908 & 2.23 & 0.023 & -6.783 & -8.071 & 0.298 & 0.109 & ${ }^{5} \mathrm{H}_{4}$ \\
\hline
\end{tabular}




\begin{tabular}{|c|c|c|c|c|c|c|c|}
\hline-1688.9288 & -1688.3926 & 1.07 & 0.163 & -6.797 & -8.065 & 0.302 & 0.101 \\
\hline-1688.9297 & -1688.3932 & 0.73 & 0.292 & -6.791 & -8.082 & 0.298 & 0.111 \\
\hline-1688.9272 & -1688.3900 & 2.73 & 0.010 & -6.784 & -7.993 & 0.304 & 0.095 \\
\hline-1688.9271 & -1688.3910 & 2.11 & 0.028 & -6.804 & -7.985 & 0.314 & 0.088 \\
\hline-1688.9276 & -1688.3928 & 0.99 & 0.189 & -6.776 & -8.079 & 0.298 & 0.110 \\
\hline-1688.9278 & -1688.3925 & 1.14 & 0.145 & -6.806 & -8.042 & 0.300 & 0.098 \\
\hline-1688.9299 & -1688.3936 & 0.45 & 0.468 & -6.807 & -7.985 & 0.295 & 0.101 \\
\hline-1688.9295 & -1688.3928 & 0.93 & 0.208 & -6.790 & -8.157 & 0.213 & 0.154 \\
\hline-1688.9287 & -1688.3923 & 1.30 & 0.111 & -6.679 & -8.124 & 0.169 & 0.167 \\
\hline-1688.9286 & -1688.3926 & 1.07 & 0.164 & -6.667 & -8.078 & 0.176 & 0.165 \\
\hline-1688.9261 & -1688.3886 & 3.58 & 0.002 & -6.769 & -8.062 & 0.327 & 0.101 \\
\hline-1688.9269 & -1688.3899 & 2.78 & 0.009 & -6.828 & -8.031 & 0.315 & 0.096 \\
\hline-1688.9293 & -1688.3930 & 0.85 & 0.238 & -6.788 & -8.074 & 0.302 & 0.105 \\
\hline-1688.9273 & -1688.3912 & 1.98 & 0.036 & -6.758 & -7.984 & 0.313 & 0.092 \\
\hline-1688.9283 & -1688.3916 & 1.71 & 0.056 & -6.796 & -7.993 & 0.295 & 0.099 \\
\hline-1688.9272 & -1688.3903 & 2.56 & 0.013 & -6.763 & -7.960 & 0.344 & 0.070 \\
\hline-1688.9285 & -1688.3920 & 1.46 & 0.085 & -6.803 & -7.982 & 0.332 & 0.082 \\
\hline-1688.9274 & -1688.3916 & 1.69 & 0.058 & -6.798 & -8.065 & 0.298 & 0.108 \\
\hline-1688.9292 & -1688.3932 & 0.71 & 0.301 & -6.771 & -8.128 & 0.292 & 0.114 \\
\hline-1688.9303 & -1688.3925 & 1.12 & 0.150 & -6.812 & -7.990 & 0.294 & 0.100 \\
\hline-1688.9299 & -1688.3936 & 0.48 & 0.447 & -6.803 & -8.069 & 0.309 & 0.098 \\
\hline-1688.9260 & -1688.3904 & 2.44 & 0.016 & -6.811 & -8.029 & 0.322 & 0.096 \\
\hline-1688.9305 & -1688.3933 & 0.63 & 0.345 & -6.787 & -7.976 & 0.296 & 0.101 \\
\hline-1688.9306 & -1688.3938 & 0.33 & 0.571 & -6.799 & -7.967 & 0.296 & 0.100 \\
\hline-1688.9264 & -1688.3901 & 2.66 & 0.011 & -6.596 & -8.126 & 0.141 & 0.171 \\
\hline
\end{tabular}

Table SCY7. Energetics for 7 at the PBE0/D3BJ/CPCM $\left(\mathrm{CH}_{3} \mathrm{CN}\right) / / \mathrm{PBE} 0 / \mathrm{D} 3 \mathrm{BJ} / \mathrm{DEF} 2-$ $\mathrm{SVP} / \mathrm{RIJCOSX}$ level. Relative energies in $\mathrm{kcal} / \mathrm{mol}$, orbital energies in $\mathrm{eV}$.

\begin{tabular}{|c|c|c|c|c|c|c|c|c|}
\hline E (a.u.) & G (a.u.) & $\Delta \mathrm{G}$ & Boltz. & HOMO & $\begin{array}{c}\pi_{C_{1}-C_{2}} \\
(\mathrm{ev})\end{array}$ & $\begin{array}{c}\Delta E^{(2)}(e V) \\
\left(\pi \rightarrow \sigma_{C_{3}-o}^{*}\right)\end{array}$ & $\begin{array}{c}\Delta E^{(2)}(e V) \\
\left(\pi \rightarrow \sigma_{C_{3}-H}^{*}\right)\end{array}$ & Conformer \\
\hline-5471.3843 & -5471.1491 & 3.13 & 0.005 & -7.206 & -8.346 & 0.382 & 0.086 & ${ }^{5} \mathrm{H}_{4}$ \\
\hline-5471.3859 & -5471.1506 & 2.15 & 0.027 & -7.250 & -8.353 & 0.373 & 0.088 & ${ }^{5} \mathrm{H}_{4}$ \\
\hline-5471.3845 & -5471.1493 & 2.98 & 0.006 & -7.235 & -8.351 & 0.374 & 0.083 & ${ }^{5} \mathrm{H}_{4}$ \\
\hline-5471.3872 & -5471.1531 & 0.59 & 0.371 & -7.233 & -8.378 & 0.385 & 0.085 & ${ }^{5} \mathrm{H}_{4}$ \\
\hline-5471.3847 & -5471.1495 & 2.87 & 0.008 & -7.204 & -8.342 & 0.369 & 0.088 & ${ }^{5} \mathrm{H}_{4}$ \\
\hline-5471.3820 & -5471.1473 & 4.26 & 0.001 & -7.074 & -8.540 & 0.379 & 0.088 & ${ }^{5} \mathrm{H}_{4}$ \\
\hline-5471.3814 & -5471.1464 & 4.84 & 0.000 & -7.058 & -8.560 & 0.389 & 0.091 & ${ }^{5} \mathrm{H}_{4}$ \\
\hline-5471.3858 & -5471.1510 & 1.92 & 0.039 & -7.083 & -8.536 & 0.372 & 0.089 & ${ }^{5} \mathrm{H}_{4}$ \\
\hline-5471.3815 & -5471.1465 & 4.76 & 0.000 & -7.026 & -8.515 & 0.376 & 0.094 & ${ }^{5} \mathrm{H}_{4}$ \\
\hline-5471.3813 & -5471.1444 & 6.04 & 0.000 & -7.049 & -8.578 & 0.374 & 0.089 & ${ }^{5} \mathrm{H}_{4}$ \\
\hline-5471.3833 & -5471.1487 & 3.40 & 0.003 & -7.106 & -8.453 & 0.368 & 0.100 & ${ }^{5} \mathrm{H}_{4}$ \\
\hline-5471.3823 & -5471.1476 & 4.08 & 0.001 & -7.026 & -8.535 & 0.372 & 0.089 & ${ }^{5} \mathrm{H}_{4}$ \\
\hline-5471.3802 & -5471.1469 & 4.51 & 0.000 & -7.038 & -8.520 & 0.379 & 0.088 & ${ }^{5} \mathrm{H}_{4}$ \\
\hline-5471.3838 & -5471.1488 & 3.31 & 0.004 & -7.087 & -8.463 & 0.372 & 0.082 & ${ }^{5} \mathrm{H}_{4}$ \\
\hline-5471.3844 & -5471.1477 & 3.97 & 0.001 & -7.061 & -8.556 & 0.379 & 0.089 & ${ }^{5} \mathrm{H}_{4}$ \\
\hline-5471.3864 & -5471.1514 & 1.67 & 0.060 & -7.247 & -8.362 & 0.386 & 0.090 & ${ }^{5} \mathrm{H}_{4}$ \\
\hline-5471.3849 & -5471.1498 & 2.68 & 0.011 & -7.226 & -8.341 & 0.384 & 0.085 & ${ }^{5} \mathrm{H}_{4}$ \\
\hline-5471.3854 & -5471.1500 & 2.54 & 0.014 & -7.236 & -8.324 & 0.379 & 0.088 & ${ }^{5} \mathrm{H}_{4}$ \\
\hline-5471.3845 & -5471.1498 & 2.67 & 0.011 & -7.242 & -8.332 & 0.368 & 0.088 & ${ }^{5} \mathrm{H}_{4}$ \\
\hline-5471.3854 & -5471.1500 & 2.56 & 0.013 & -7.198 & -8.382 & 0.378 & 0.092 & ${ }^{5} \mathrm{H}_{4}$ \\
\hline-5471.3829 & -5471.1484 & 3.53 & 0.003 & -7.076 & -8.516 & 0.377 & 0.093 & ${ }^{5} \mathrm{H}_{4}$ \\
\hline-5471.3833 & -5471.1475 & 4.11 & 0.001 & -7.059 & -8.553 & 0.369 & 0.085 & ${ }^{5} \mathrm{H}_{4}$ \\
\hline-5471.3869 & -5471.1510 & 1.91 & 0.040 & -7.055 & -8.496 & 0.383 & 0.086 & ${ }^{5} \mathrm{H}_{4}$ \\
\hline-5471.3811 & -5471.1465 & 4.78 & 0.000 & -7.032 & -8.521 & 0.376 & 0.091 & ${ }^{5} \mathrm{H}_{4}$ \\
\hline-5471.3853 & -5471.1496 & 2.81 & 0.009 & -7.038 & -8.624 & 0.368 & 0.084 & ${ }^{5} \mathrm{H}_{4}$ \\
\hline-5471.3843 & -5471.1482 & 3.68 & 0.002 & -7.077 & -8.595 & 0.359 & 0.106 & ${ }^{5} \mathrm{H}_{4}$ \\
\hline-5471.3799 & -5471.1440 & 6.35 & 0.000 & -7.044 & -8.544 & 0.379 & 0.094 & ${ }^{5} \mathrm{H}_{4}$ \\
\hline-5471.3815 & -5471.1465 & 4.73 & 0.000 & -7.026 & -8.516 & 0.371 & 0.094 & ${ }^{5} \mathrm{H}_{4}$ \\
\hline-5471.3839 & -5471.1476 & 4.05 & 0.001 & -7.075 & -8.559 & 0.383 & 0.086 & ${ }^{5} \mathrm{H}_{4}$ \\
\hline-5471.3833 & -5471.1477 & 4.01 & 0.001 & -7.063 & -8.575 & 0.387 & 0.093 & ${ }^{5} \mathrm{H}_{4}$ \\
\hline
\end{tabular}




\begin{tabular}{|c|c|c|c|c|c|c|c|c|}
\hline-5471.3845 & -5471.1484 & 3.54 & 0.003 & -7.182 & -8.361 & 0.378 & 0.092 & ${ }^{5} \mathrm{H}_{4}$ \\
\hline-5471.3836 & -5471.1486 & 3.44 & 0.003 & -7.241 & -8.360 & 0.370 & 0.094 & ${ }^{5} \mathrm{H}_{4}$ \\
\hline-5471.3844 & -5471.1491 & 3.09 & 0.005 & -7.203 & -8.344 & 0.378 & 0.092 & ${ }^{5} \mathrm{H}_{4}$ \\
\hline-5471.3870 & -5471.1529 & 0.73 & 0.290 & -7.232 & -8.373 & 0.367 & 0.086 & ${ }^{5} \mathrm{H}_{4}$ \\
\hline-5471.3835 & -5471.1473 & 4.23 & 0.001 & -7.051 & -8.531 & 0.385 & 0.086 & ${ }^{5} \mathrm{H}_{4}$ \\
\hline-5471.3801 & -5471.1443 & 6.16 & 0.000 & -7.011 & -8.577 & 0.224 & 0.150 & ${ }^{4} \mathrm{H}_{5}$ \\
\hline-5471.3872 & -5471.1525 & 0.96 & 0.198 & -7.109 & -8.426 & 0.220 & 0.151 & ${ }^{4} \mathrm{H}_{5}$ \\
\hline-5471.3821 & -5471.1476 & 4.07 & 0.001 & -7.031 & -8.529 & 0.225 & 0.149 & ${ }^{4} \mathrm{H}_{5}$ \\
\hline-5471.3836 & -5471.1474 & 4.21 & 0.001 & -7.085 & -8.579 & 0.229 & 0.149 & ${ }^{4} \mathrm{H}_{5}$ \\
\hline-5471.3904 & -5471.1541 & 0.00 & 1.000 & -7.069 & -8.557 & 0.367 & 0.086 & ${ }^{5} \mathrm{H}_{4}$ \\
\hline-5471.3823 & -5471.1463 & 4.88 & 0.000 & -7.044 & -8.579 & 0.211 & 0.157 & ${ }^{4} \mathrm{H}_{5}$ \\
\hline-5471.3855 & -5471.1500 & 2.58 & 0.013 & -7.038 & -8.630 & 0.215 & 0.157 & ${ }^{4} \mathrm{H}_{5}$ \\
\hline-5471.3846 & -5471.1488 & 3.29 & 0.004 & -7.080 & -8.545 & 0.236 & 0.153 & ${ }^{4} \mathrm{H}_{5}$ \\
\hline-5471.3855 & -5471.1499 & 2.60 & 0.012 & -7.040 & -8.625 & 0.229 & 0.161 & ${ }^{4} \mathrm{H}_{5}$ \\
\hline-5471.3865 & -5471.1515 & 1.63 & 0.064 & -7.248 & -8.364 & 0.221 & 0.151 & ${ }^{4} \mathrm{H}_{5}$ \\
\hline-5471.3849 & -5471.1493 & 2.98 & 0.007 & -7.189 & -8.334 & 0.223 & 0.152 & ${ }^{4} \mathrm{H}_{5}$ \\
\hline-5471.3866 & -5471.1527 & 0.88 & 0.226 & -7.225 & -8.368 & 0.216 & 0.153 & ${ }^{4} \mathrm{H}_{5}$ \\
\hline-5471.3862 & -5471.1505 & 2.22 & 0.023 & -7.204 & -8.350 & 0.229 & 0.149 & ${ }^{4} \mathrm{H}_{5}$ \\
\hline-5471.3862 & -5471.1507 & 2.09 & 0.029 & -7.240 & -8.341 & 0.222 & 0.152 & ${ }^{4} \mathrm{H}_{5}$ \\
\hline-5471.3833 & -5471.1471 & 4.35 & 0.001 & -7.058 & -8.541 & 0.196 & 0.159 & ${ }^{4} \mathrm{H}_{5}$ \\
\hline-5471.3792 & -5471.1444 & 6.04 & 0.000 & -7.063 & -8.550 & 0.216 & 0.157 & ${ }^{4} \mathrm{H}_{5}$ \\
\hline-5471.3826 & -5471.1466 & 4.70 & 0.000 & -7.046 & -8.581 & 0.223 & 0.156 & ${ }^{4} \mathrm{H}_{5}$ \\
\hline-5471.3865 & -5471.1499 & 2.64 & 0.012 & -7.049 & -8.518 & 0.213 & 0.161 & ${ }^{4} \mathrm{H}_{5}$ \\
\hline-5471.3831 & -5471.1475 & 4.14 & 0.001 & -7.027 & -8.524 & 0.225 & 0.149 & ${ }^{4} \mathrm{H}_{5}$ \\
\hline-5471.3835 & -5471.1475 & 4.13 & 0.001 & -7.059 & -8.558 & 0.227 & 0.162 & ${ }^{4} \mathrm{H}_{5}$ \\
\hline-5471.3859 & -5471.1507 & 2.14 & 0.027 & -7.068 & -8.496 & 0.223 & 0.156 & ${ }^{4} \mathrm{H}_{5}$ \\
\hline-5471.3795 & -5471.1434 & 6.70 & 0.000 & -7.022 & -8.546 & 0.221 & 0.156 & ${ }^{4} \mathrm{H}_{5}$ \\
\hline-5471.3848 & -5471.1490 & 3.15 & 0.005 & -7.083 & -8.539 & 0.214 & 0.155 & ${ }^{4} \mathrm{H}_{5}$ \\
\hline-5471.3850 & -5471.1495 & 2.86 & 0.008 & -7.242 & -8.332 & 0.226 & 0.149 & ${ }^{4} \mathrm{H}_{5}$ \\
\hline-5471.3871 & -5471.1531 & 0.62 & 0.352 & -7.229 & -8.379 & 0.221 & 0.151 & ${ }^{4} \mathrm{H}_{5}$ \\
\hline-5471.3853 & -5471.1501 & 2.51 & 0.014 & -7.201 & -8.338 & 0.224 & 0.149 & ${ }^{4} \mathrm{H}_{5}$ \\
\hline-5471.3845 & -5471.1498 & 2.66 & 0.011 & -7.238 & -8.327 & 0.222 & 0.151 & ${ }^{4} \mathrm{H}_{5}$ \\
\hline-5471.3873 & -5471.1533 & 0.50 & 0.430 & -7.230 & -8.372 & 0.218 & 0.157 & ${ }^{4} \mathrm{H}_{5}$ \\
\hline-5471.3869 & -5471.1526 & 0.89 & 0.222 & -7.069 & -8.510 & 0.228 & 0.148 & ${ }^{4} \mathrm{H}_{5}$ \\
\hline-5471.3835 & -5471.1475 & 4.12 & 0.001 & -7.058 & -8.558 & 0.219 & 0.154 & ${ }^{4} \mathrm{H}_{5}$ \\
\hline-5471.3852 & -5471.1514 & 1.67 & 0.059 & -7.137 & -8.444 & 0.225 & 0.149 & ${ }^{4} \mathrm{H}_{5}$ \\
\hline-5471.3866 & -5471.1510 & 1.91 & 0.040 & -7.060 & -8.493 & 0.223 & 0.152 & ${ }^{4} \mathrm{H}_{5}$ \\
\hline-5471.3864 & -5471.1513 & 1.76 & 0.051 & -7.057 & -8.529 & 0.217 & 0.157 & ${ }^{4} \mathrm{H}_{5}$ \\
\hline-5471.3832 & -5471.1475 & 4.11 & 0.001 & -7.026 & -8.531 & 0.230 & 0.160 & ${ }^{4} \mathrm{H}_{5}$ \\
\hline-5471.3846 & -5471.1488 & 3.29 & 0.004 & -7.081 & -8.538 & 0.194 & 0.160 & ${ }^{4} \mathrm{H}_{5}$ \\
\hline-5471.3842 & -5471.1489 & 3.24 & 0.004 & -7.049 & -8.521 & 0.222 & 0.156 & ${ }^{4} \mathrm{H}_{5}$ \\
\hline-5471.3847 & -5471.1489 & 3.21 & 0.004 & -7.082 & -8.541 & 0.214 & 0.157 & ${ }^{4} \mathrm{H}_{5}$ \\
\hline-5471.3780 & -5471.1415 & 7.89 & 0.000 & -7.033 & -8.534 & 0.219 & 0.157 & ${ }^{4} \mathrm{H}_{5}$ \\
\hline-5471.3845 & -5471.1492 & 3.06 & 0.006 & -7.203 & -8.339 & 0.214 & 0.158 & ${ }^{4} \mathrm{H}_{5}$ \\
\hline-5471.3847 & -5471.1507 & 2.10 & 0.029 & -7.266 & -8.369 & 0.218 & 0.153 & ${ }^{4} \mathrm{H}_{5}$ \\
\hline-5471.3842 & -5471.1488 & 3.33 & 0.004 & -7.234 & -8.352 & 0.223 & 0.150 & ${ }^{4} \mathrm{H}_{5}$ \\
\hline-5471.3852 & -5471.1500 & 2.57 & 0.013 & -7.176 & -8.367 & 0.224 & 0.148 & ${ }^{4} \mathrm{H}_{5}$ \\
\hline-5471.3860 & -5471.1505 & 2.23 & 0.023 & -7.240 & -8.340 & 0.217 & 0.156 & ${ }^{4} \mathrm{H}_{5}$ \\
\hline-5471.3880 & -5471.1540 & 0.05 & 0.921 & -7.076 & -8.532 & 0.216 & 0.155 & ${ }^{4} \mathrm{H}_{5}$ \\
\hline-5471.3851 & -5471.1497 & 2.76 & 0.009 & -7.086 & -8.572 & 0.226 & 0.147 & ${ }^{4} \mathrm{H}_{5}$ \\
\hline-5471.3847 & -5471.1493 & 3.00 & 0.006 & -7.070 & -8.526 & 0.224 & 0.148 & ${ }^{4} \mathrm{H}_{5}$ \\
\hline-5471.3841 & -5471.1484 & 3.55 & 0.003 & -7.061 & -8.528 & 0.222 & 0.151 & ${ }^{4} \mathrm{H}_{5}$ \\
\hline-5471.3848 & -5471.1486 & 3.43 & 0.003 & -7.076 & -8.529 & 0.226 & 0.148 & ${ }^{4} \mathrm{H}_{5}$ \\
\hline-5471.3844 & -5471.1477 & 3.97 & 0.001 & -7.062 & -8.552 & 0.222 & 0.152 & ${ }^{4} \mathrm{H}_{5}$ \\
\hline-5471.3844 & -5471.1477 & 4.03 & 0.001 & -7.060 & -8.556 & 0.222 & 0.150 & ${ }^{4} \mathrm{H}_{5}$ \\
\hline-5471.3834 & -5471.1471 & 4.34 & 0.001 & -7.053 & -8.536 & 0.230 & 0.153 & ${ }^{4} \mathrm{H}_{5}$ \\
\hline-5471.3844 & -5471.1485 & 3.50 & 0.003 & -7.158 & -8.339 & 0.224 & 0.151 & ${ }^{4} \mathrm{H}_{5}$ \\
\hline-5471.3858 & -5471.1506 & 2.17 & 0.026 & -7.250 & -8.352 & 0.222 & 0.152 & ${ }^{4} \mathrm{H}_{5}$ \\
\hline-5471.3876 & -5471.1527 & 0.85 & 0.240 & -7.206 & -8.359 & 0.220 & 0.152 & ${ }^{4} \mathrm{H}_{5}$ \\
\hline-5471.3846 & -5471.1493 & 2.97 & 0.007 & -7.203 & -8.342 & 0.219 & 0.153 & ${ }^{4} \mathrm{H}_{5}$ \\
\hline-5471.3740 & -5471.1381 & 10.02 & 0.000 & -7.040 & -8.536 & 0.234 & 0.153 & ${ }^{4} \mathrm{H}_{5}$ \\
\hline-5471.3836 & -5471.1473 & 4.23 & 0.001 & -7.085 & -8.582 & 0.224 & 0.151 & ${ }^{4} \mathrm{H}_{5}$ \\
\hline
\end{tabular}




$\begin{array}{lllllllll}-5471.3840 & -5471.1481 & 3.76 & 0.002 & -7.074 & -8.557 & 0.226 & 0.149 & { }^{4} \mathrm{H}_{5} \\ -5471.3828 & -5471.1481 & 3.75 & 0.002 & -7.090 & -8.535 & 0.197 & 0.158 & { }^{4} \mathrm{H}_{5} \\ -5471.3810 & -5471.1459 & 5.12 & 0.000 & -7.051 & -8.571 & 0.219 & 0.157 & { }^{4} \mathrm{H}_{5} \\ -5471.3754 & -5471.1393 & 9.24 & 0.000 & -7.041 & -8.539 & 0.213 & 0.160 & { }^{4} \mathrm{H}_{5} \\ -5471.3827 & -5471.1470 & 4.45 & 0.001 & -7.013 & -8.535 & 0.218 & 0.153 & { }^{4} \mathrm{H}_{5} \\ -5471.3863 & -5471.1514 & 1.71 & 0.056 & -7.249 & -8.366 & 0.225 & 0.149 & { }^{4} \mathrm{H}_{5} \\ -5471.3872 & -5471.1532 & 0.55 & 0.397 & -7.232 & -8.372 & 0.213 & 0.162 & { }^{4} \mathrm{H}_{5} \\ -5471.3869 & -5471.1516 & 1.53 & 0.076 & -7.212 & -8.357 & 0.223 & 0.162 & { }^{4} \mathrm{H}_{5} \\ -5471.3818 & -5471.1473 & 4.25 & 0.001 & -7.077 & -8.525 & 0.216 & 0.155 & { }^{4} \mathrm{H}_{5} \\ -5471.3833 & -5471.1485 & 3.49 & 0.003 & -7.105 & -8.449 & 0.219 & 0.153 & { }^{4} \mathrm{H}_{5} \\ -5471.3856 & -5471.1502 & 2.43 & 0.017 & -7.040 & -8.624 & 0.218 & 0.150 & { }^{4} \mathrm{H}_{5} \\ -5471.3826 & -5471.1473 & 4.23 & 0.001 & -7.074 & -8.576 & 0.220 & 0.153 & { }^{4} \mathrm{H}_{5} \\ -5471.3786 & -5471.1439 & 6.40 & 0.000 & -7.052 & -8.543 & 0.218 & 0.153 & { }^{4} \mathrm{H}_{5} \\ -5471.3845 & -5471.1497 & 2.74 & 0.010 & -7.067 & -8.509 & 0.216 & 0.153 & { }^{4} \mathrm{H}_{5} \\ -5471.3818 & -5471.1473 & 4.23 & 0.001 & -7.032 & -8.529 & 0.197 & 0.159 & { }^{4} \mathrm{H}_{5} \\ -5471.3835 & -5471.1476 & 4.07 & 0.001 & -7.056 & -8.559 & 0.221 & 0.153 & { }^{4} \mathrm{H}_{5} \\ -5471.3835 & -5471.1476 & 4.09 & 0.001 & -7.058 & -8.556 & 0.229 & 0.147 & { }^{4} \mathrm{H}_{5} \\ -5471.3822 & -5471.1475 & 4.14 & 0.001 & -7.101 & -8.446 & 0.229 & 0.149 & { }^{4} \mathrm{H}_{5}\end{array}$

Table SCY8. Energetics for 9 at the PBE0/D3BJ/CPCM $\left(\mathrm{CH}_{3} \mathrm{CN}\right) / / \mathrm{PBE} 0 / \mathrm{D} 3 \mathrm{BJ} / \mathrm{DEF} 2-$ $\mathrm{SVP} / \mathrm{RIJCOSX}$ level. Relative energies in $\mathrm{kcal} / \mathrm{mol}$, orbital energies in $\mathrm{eV}$.

\begin{tabular}{|c|c|c|c|c|c|c|c|c|}
\hline E (a.u.) & G (a.u.) & $\Delta \mathrm{G}$ & Boltz. & HOMO & $\begin{array}{c}\pi_{C_{1}-C_{2}} \\
(\mathrm{ev})\end{array}$ & $\begin{array}{c}\Delta E^{(2)}(e V) \\
\left(\pi \rightarrow \sigma_{C_{3}-O}^{*}\right)\end{array}$ & $\begin{array}{c}\Delta E^{(2)}(e V) \\
\left(\pi \rightarrow \sigma_{C_{3}-H}^{*}\right)\end{array}$ & Conformer \\
\hline-1419.5797 & -1419.1539 & 6.95 & 0.000 & -6.815 & -8.060 & 0.318 & 0.093 & ${ }^{5} \mathrm{H}_{4}$ \\
\hline-1419.5792 & -1419.1536 & 7.13 & 0.000 & -6.779 & -8.019 & 0.335 & 0.075 & ${ }^{5} \mathrm{H}_{4}$ \\
\hline-1419.5789 & -1419.1549 & 6.30 & 0.000 & -6.832 & -8.062 & 0.323 & 0.090 & ${ }^{5} \mathrm{H}_{4}$ \\
\hline-1419.5799 & -1419.1552 & 6.09 & 0.000 & -6.918 & -8.093 & 0.349 & 0.069 & ${ }^{5} \mathrm{H}_{4}$ \\
\hline-1419.5906 & -1419.1649 & 0.00 & 1.000 & -7.037 & -8.190 & 0.307 & 0.100 & ${ }^{5} \mathrm{H}_{4}$ \\
\hline-1419.5795 & -1419.1548 & 6.37 & 0.000 & -6.904 & -8.100 & 0.351 & 0.072 & ${ }^{5} \mathrm{H}_{4}$ \\
\hline-1419.5822 & -1419.1576 & 4.58 & 0.000 & -6.885 & -8.096 & 0.309 & 0.097 & ${ }^{5} \mathrm{H}_{4}$ \\
\hline-1419.5822 & -1419.1565 & 5.31 & 0.000 & -6.838 & -8.112 & 0.302 & 0.094 & ${ }^{5} \mathrm{H}_{4}$ \\
\hline-1419.5829 & -1419.1581 & 4.30 & 0.001 & -7.022 & -8.116 & 0.311 & 0.087 & ${ }^{5} \mathrm{H}_{4}$ \\
\hline-1419.5809 & -1419.1575 & 4.66 & 0.000 & -7.000 & -8.098 & 0.306 & 0.087 & ${ }^{5} \mathrm{H}_{4}$ \\
\hline-1419.5811 & -1419.1559 & 5.70 & 0.000 & -6.903 & -8.111 & 0.348 & 0.072 & ${ }^{5} \mathrm{H}_{4}$ \\
\hline-1419.5822 & -1419.1564 & 5.35 & 0.000 & -6.943 & -8.118 & 0.318 & 0.088 & ${ }^{5} \mathrm{H}_{4}$ \\
\hline-1419.5806 & -1419.1552 & 6.08 & 0.000 & -6.923 & -8.129 & 0.350 & 0.080 & ${ }^{5} \mathrm{H}_{4}$ \\
\hline-1419.5825 & -1419.1567 & 5.18 & 0.000 & -6.843 & -8.117 & 0.301 & 0.095 & ${ }^{5} \mathrm{H}_{4}$ \\
\hline-1419.5821 & -1419.1563 & 5.40 & 0.000 & -6.837 & -8.113 & 0.300 & 0.095 & ${ }^{5} \mathrm{H}_{4}$ \\
\hline
\end{tabular}

Table SCY9. Energetics for 10 at the PBE0/D3BJ/CPCM $\left(\mathrm{CH}_{3} \mathrm{CN}\right) / / \mathrm{PBE} 0 / \mathrm{D} 3 \mathrm{BJ} / \mathrm{DEF} 2-$ SVP/RIJCOSX level. Relative energies in $\mathrm{kcal} / \mathrm{mol}$, orbital energies in $\mathrm{eV}$.

$\begin{array}{ccccccccc}\text { E (a.u.) } & \mathrm{G} \text { (a.u.) } & \Delta \mathrm{G} & \text { Boltz. } & \mathrm{HOMO} & \begin{array}{c}\pi_{C_{1}-C_{2}} \\ (\mathrm{ev})\end{array} & \begin{array}{c}\Delta E^{(2)}(\mathrm{eV}) \\ \left(\pi \rightarrow \sigma_{C_{3}}^{*} \mathrm{O}\right)\end{array} & \begin{array}{c}\Delta E^{(2)}(\mathrm{eV}) \\ \left(\pi \rightarrow \sigma_{C_{3}-\mathrm{H}}^{*}\right)\end{array} & \text { Conformer } \\ -1484.4543 & -1483.9959 & 0.31 & 0.598 & -6.875 & -8.171 & 0.352 & 0.086 & { }^{5} \mathrm{H}_{4} \\ -1484.4504 & -1483.9912 & 3.21 & 0.004 & -6.871 & -8.161 & 0.352 & 0.085 & { }^{5} \mathrm{H}_{4} \\ -1484.4503 & -1483.9912 & 3.23 & 0.004 & -6.871 & -8.163 & 0.353 & 0.085 & { }^{5} \mathrm{H}_{4} \\ -1484.4519 & -1483.9928 & 2.23 & 0.023 & -6.869 & -8.171 & 0.353 & 0.089 & { }^{5} \mathrm{H}_{4} \\ -1484.4530 & -1483.9936 & 1.73 & 0.054 & -6.866 & -8.171 & 0.352 & 0.087 & { }^{5} \mathrm{H}_{4} \\ -1484.4544 & -1483.9958 & 0.35 & 0.558 & -6.870 & -8.174 & 0.352 & 0.087 & { }^{5} \mathrm{H}_{4} \\ -1484.4517 & -1483.9940 & 1.49 & 0.080 & -6.877 & -8.179 & 0.352 & 0.091 & { }^{5} \mathrm{H}_{4} \\ -1484.4521 & -1483.9926 & 2.33 & 0.020 & -6.875 & -8.162 & 0.352 & 0.083 & { }^{5} \mathrm{H}_{4} \\ -1484.4515 & -1483.9923 & 2.51 & 0.014 & -6.876 & -8.173 & 0.353 & 0.086 & { }^{5} \mathrm{H}_{4} \\ -1484.4538 & -1483.9961 & 0.17 & 0.747 & -6.919 & -8.230 & 0.382 & 0.087 & { }^{5} \mathrm{H}_{4} \\ -1484.4519 & -1483.9927 & 2.29 & 0.021 & -6.864 & -8.165 & 0.352 & 0.085 & { }^{5} \mathrm{H}_{4} \\ -1484.4528 & -1483.9943 & 1.31 & 0.109 & -6.873 & -8.166 & 0.350 & 0.086 & { }^{5} \mathrm{H}_{4} \\ -1484.4513 & -1483.9930 & 2.09 & 0.029 & -6.875 & -8.178 & 0.352 & 0.091 & { }^{5} \mathrm{H}_{4} \\ -1484.4516 & -1483.9936 & 1.72 & 0.055 & -6.873 & -8.172 & 0.352 & 0.089 & { }^{5} \mathrm{H}_{4} \\ -1484.4543 & -1483.9957 & 0.40 & 0.505 & -6.945 & -8.250 & 0.360 & 0.089 & { }^{5} \mathrm{H}_{4}\end{array}$




$\begin{array}{lllllllll}-1484.4514 & -1483.9922 & 2.57 & 0.013 & -6.865 & -8.160 & 0.352 & 0.085 & { }^{5} \mathrm{H}_{4} \\ -1484.4530 & -1483.9959 & 0.30 & 0.603 & -6.938 & -8.222 & 0.384 & 0.087 & { }^{5} \mathrm{H}_{4} \\ -1484.4531 & -1483.9941 & 1.42 & 0.091 & -6.876 & -8.159 & 0.353 & 0.083 & { }^{5} \mathrm{H}_{4} \\ -1484.4520 & -1483.9924 & 2.44 & 0.016 & -6.876 & -8.160 & 0.352 & 0.083 & { }^{5} \mathrm{H}_{4} \\ -1484.4535 & -1483.9963 & 0.00 & 1.000 & -6.930 & -8.227 & 0.383 & 0.089 & { }^{5} \mathrm{H}_{4} \\ -1484.4518 & -1483.9936 & 1.70 & 0.057 & -6.873 & -8.160 & 0.353 & 0.085 & { }^{5} \mathrm{H}_{4} \\ -1484.4491 & -1483.9906 & 3.61 & 0.002 & -6.875 & -8.173 & 0.358 & 0.084 & { }^{5} \mathrm{H}_{4} \\ -1484.4525 & -1483.9941 & 1.43 & 0.089 & -6.857 & -8.177 & 0.349 & 0.090 & { }^{5} \mathrm{H}_{4} \\ -1484.4529 & -1483.9945 & 1.15 & 0.143 & -6.874 & -8.162 & 0.353 & 0.085 & { }^{5} \mathrm{H}_{4} \\ -1484.4517 & -1483.9931 & 2.06 & 0.031 & -6.861 & -8.159 & 0.353 & 0.084 & { }^{5} \mathrm{H}_{4} \\ -1484.4540 & -1483.9954 & 0.60 & 0.363 & -6.883 & -8.181 & 0.353 & 0.088 & { }^{5} \mathrm{H}_{4} \\ -1484.4532 & -1483.9940 & 1.50 & 0.080 & -6.872 & -8.175 & 0.352 & 0.087 & { }^{5} \mathrm{H}_{4} \\ -1484.4517 & -1483.9942 & 1.36 & 0.101 & -6.878 & -8.189 & 0.351 & 0.093 & { }^{5} \mathrm{H}_{4}\end{array}$

Table SCY10. Energetics for 11 at the PBE0/D3BJ/CPCM $\left(\mathrm{CH}_{3} \mathrm{CN}\right) / / \mathrm{PBE} 0 / \mathrm{D} 3 \mathrm{BJ} / \mathrm{DEF} 2-$ $\mathrm{SVP} / \mathrm{RIJCOSX}$ level. Relative energies in $\mathrm{kcal} / \mathrm{mol}$, orbital energies in $\mathrm{eV}$.

\begin{tabular}{|c|c|c|c|c|c|c|c|c|}
\hline E (a.u.) & G (a.u.) & $\Delta \mathrm{G}$ & Boltz. & $\begin{array}{c}\text { HOMO } \\
-2\end{array}$ & $\begin{array}{c}\pi_{C_{1}-C_{2}} \\
(\mathrm{ev})\end{array}$ & $\begin{array}{c}\Delta E^{(2)}(e V) \\
\left(\pi \rightarrow \sigma_{C_{3}-o}^{*}\right)\end{array}$ & $\begin{array}{c}\Delta E^{(2)}(e V) \\
\left(\pi \rightarrow \sigma_{C_{3}-H}^{*}\right)\end{array}$ & Conformer \\
\hline-1948.5148 & -1947.8556 & 1.87 & 0.042 & -6.706 & -8.039 & 0.306 & 0.102 & ${ }^{5} \mathrm{H}_{4}$ \\
\hline-1948.5145 & -1947.8551 & 2.15 & 0.027 & -6.732 & -8.054 & 0.297 & 0.109 & ${ }^{5} \mathrm{H}_{4}$ \\
\hline-1948.5134 & -1947.8543 & 2.69 & 0.011 & -6.728 & -8.055 & 0.297 & 0.108 & ${ }^{5} \mathrm{H}_{4}$ \\
\hline-1948.5154 & -1947.8555 & 1.91 & 0.040 & -6.779 & -7.984 & 0.323 & 0.090 & ${ }^{5} \mathrm{H}_{4}$ \\
\hline-1948.5148 & -1947.8539 & 2.93 & 0.007 & -6.757 & -7.980 & 0.311 & 0.096 & ${ }^{5} \mathrm{H}_{4}$ \\
\hline-1948.5150 & -1947.8557 & 1.77 & 0.051 & -6.703 & -7.953 & 0.313 & 0.091 & ${ }^{5} \mathrm{H}_{4}$ \\
\hline-1948.5142 & -1947.8550 & 2.23 & 0.023 & -6.768 & -8.047 & 0.312 & 0.100 & ${ }^{5} \mathrm{H}_{4}$ \\
\hline-1948.5158 & -1947.8556 & 1.84 & 0.045 & -6.750 & -7.976 & 0.318 & 0.092 & ${ }^{5} \mathrm{H}_{4}$ \\
\hline-1948.5139 & -1947.8543 & 2.66 & 0.011 & -6.720 & -8.086 & 0.288 & 0.116 & ${ }^{5} \mathrm{H}_{4}$ \\
\hline-1948.5161 & -1947.8559 & 1.66 & 0.060 & -6.768 & -7.994 & 0.304 & 0.098 & ${ }^{5} \mathrm{H}_{4}$ \\
\hline-1948.5149 & -1947.8548 & 2.36 & 0.018 & -6.723 & -8.053 & 0.299 & 0.108 & ${ }^{5} \mathrm{H}_{4}$ \\
\hline-1948.5108 & -1947.8522 & 3.98 & 0.001 & -6.764 & -7.960 & 0.324 & 0.092 & ${ }^{5} \mathrm{H}_{4}$ \\
\hline-1948.5130 & -1947.8548 & 2.36 & 0.019 & -6.753 & -8.016 & 0.330 & 0.098 & ${ }^{5} \mathrm{H}_{4}$ \\
\hline-1948.5161 & -1947.8551 & 2.17 & 0.026 & -6.713 & -7.952 & 0.317 & 0.091 & ${ }^{5} \mathrm{H}_{4}$ \\
\hline-1948.5150 & -1947.8544 & 2.59 & 0.013 & -6.759 & -8.007 & 0.296 & 0.101 & ${ }^{5} \mathrm{H}_{4}$ \\
\hline-1948.5129 & -1947.8533 & 3.27 & 0.004 & -6.719 & -7.953 & 0.318 & 0.091 & ${ }^{5} \mathrm{H}_{4}$ \\
\hline-1948.5134 & -1947.8546 & 2.50 & 0.015 & -6.766 & -8.003 & 0.320 & 0.093 & ${ }^{5} \mathrm{H}_{4}$ \\
\hline-1948.5151 & -1947.8558 & 1.71 & 0.056 & -6.734 & -8.062 & 0.296 & 0.111 & ${ }^{5} \mathrm{H}_{4}$ \\
\hline-1948.5150 & -1947.8557 & 1.81 & 0.047 & -6.734 & -8.061 & 0.296 & 0.111 & ${ }^{5} \mathrm{H}_{4}$ \\
\hline-1948.5135 & -1947.8551 & 2.20 & 0.024 & -6.761 & -8.027 & 0.320 & 0.098 & ${ }^{5} \mathrm{H}_{4}$ \\
\hline-1948.5145 & -1947.8554 & 1.95 & 0.037 & -6.772 & -8.049 & 0.310 & 0.100 & ${ }^{5} \mathrm{H}_{4}$ \\
\hline-1948.5169 & -1947.8562 & 1.45 & 0.086 & -6.746 & -7.994 & 0.300 & 0.098 & ${ }^{5} \mathrm{H}_{4}$ \\
\hline-1948.5129 & -1947.8547 & 2.39 & 0.018 & -6.642 & -8.093 & 0.168 & 0.163 & ${ }^{4} \mathrm{H}_{5}$ \\
\hline-1948.5154 & -1947.8558 & 1.73 & 0.054 & -6.694 & -8.024 & 0.307 & 0.100 & ${ }^{5} \mathrm{H}_{4}$ \\
\hline-1948.5147 & -1947.8547 & 2.41 & 0.017 & -6.726 & -8.047 & 0.300 & 0.107 & ${ }^{5} \mathrm{H}_{4}$ \\
\hline-1948.5125 & -1947.8531 & 3.43 & 0.003 & -6.727 & -7.951 & 0.322 & 0.088 & ${ }^{5} \mathrm{H}_{4}$ \\
\hline-1948.5115 & -1947.8532 & 3.37 & 0.003 & -6.722 & -7.936 & 0.340 & 0.077 & ${ }^{5} \mathrm{H}_{4}$ \\
\hline-1948.5135 & -1947.8550 & 2.24 & 0.023 & -6.757 & -8.027 & 0.320 & 0.098 & ${ }^{5} \mathrm{H}_{4}$ \\
\hline-1948.5141 & -1947.8538 & 3.02 & 0.006 & -6.780 & -7.968 & 0.311 & 0.095 & ${ }^{5} \mathrm{H}_{4}$ \\
\hline-1948.5144 & -1947.8557 & 1.82 & 0.047 & -6.771 & -7.981 & 0.336 & 0.084 & ${ }^{5} \mathrm{H}_{4}$ \\
\hline-1948.5134 & -1947.8536 & 3.13 & 0.005 & -6.721 & -8.059 & 0.296 & 0.110 & ${ }^{5} \mathrm{H}_{4}$ \\
\hline-1948.5133 & -1947.8540 & 2.87 & 0.008 & -6.720 & -8.052 & 0.298 & 0.108 & ${ }^{5} \mathrm{H}_{4}$ \\
\hline-1948.5141 & -1947.8557 & 1.80 & 0.048 & -6.768 & -8.045 & 0.311 & 0.101 & ${ }^{5} \mathrm{H}_{4}$ \\
\hline-1948.5139 & -1947.8550 & 2.25 & 0.022 & -6.768 & -8.043 & 0.314 & 0.099 & ${ }^{5} \mathrm{H}_{4}$ \\
\hline-1948.5137 & -1947.8541 & 2.82 & 0.009 & -6.716 & -8.080 & 0.288 & 0.116 & ${ }^{5} \mathrm{H}_{4}$ \\
\hline-1948.5149 & -1947.8561 & 1.57 & 0.071 & -6.710 & -8.041 & 0.305 & 0.103 & ${ }^{5} \mathrm{H}_{4}$ \\
\hline-1948.5162 & -1947.8563 & 1.42 & 0.092 & -6.695 & -7.953 & 0.317 & 0.089 & ${ }^{5} \mathrm{H}_{4}$ \\
\hline-1948.5132 & -1947.8527 & 3.67 & 0.002 & -6.758 & -8.004 & 0.322 & 0.091 & ${ }^{5} \mathrm{H}_{4}$ \\
\hline-1948.5153 & -1947.8549 & 2.29 & 0.021 & -6.712 & -7.947 & 0.319 & 0.089 & ${ }^{5} \mathrm{H}_{4}$ \\
\hline-1948.5139 & -1947.8554 & 2.01 & 0.034 & -6.741 & -7.973 & 0.316 & 0.097 & ${ }^{5} \mathrm{H}_{4}$ \\
\hline-1948.5139 & -1947.8541 & 2.83 & 0.008 & -6.691 & -7.940 & 0.319 & 0.088 & ${ }^{5} \mathrm{H}_{4}$ \\
\hline-1948.5150 & -1947.8552 & 2.13 & 0.027 & -6.714 & -7.953 & 0.320 & 0.089 & ${ }^{5} \mathrm{H}_{4}$ \\
\hline
\end{tabular}




\begin{tabular}{|c|c|c|c|c|c|c|c|c|}
\hline-1948.5122 & -1947.8514 & 4.48 & 0.001 & -6.708 & -7.929 & 0.334 & 0.075 & ${ }^{5} \mathrm{H}_{4}$ \\
\hline-1948.5139 & -1947.8552 & 2.13 & 0.027 & -6.771 & -8.041 & 0.312 & 0.099 & ${ }^{5} \mathrm{H}_{4}$ \\
\hline-1948.5147 & -1947.8543 & 2.66 & 0.011 & -6.803 & -7.982 & 0.320 & 0.094 & ${ }^{5} \mathrm{H}_{4}$ \\
\hline-1948.5155 & -1947.8565 & 1.32 & 0.108 & -6.798 & -7.984 & 0.327 & 0.090 & ${ }^{5} \mathrm{H}_{4}$ \\
\hline-1948.5158 & -1947.8560 & 1.63 & 0.064 & -6.767 & -7.969 & 0.309 & 0.095 & ${ }^{5} \mathrm{H}_{4}$ \\
\hline-1948.5152 & -1947.8542 & 2.74 & 0.010 & -6.704 & -7.982 & 0.315 & 0.093 & ${ }^{5} \mathrm{H}_{4}$ \\
\hline-1948.5165 & -1947.8567 & 1.15 & 0.143 & -6.759 & -7.990 & 0.301 & 0.097 & ${ }^{5} \mathrm{H}_{4}$ \\
\hline-1948.5162 & -1947.8560 & 1.61 & 0.066 & -6.728 & -7.984 & 0.307 & 0.098 & ${ }^{5} \mathrm{H}_{4}$ \\
\hline-1948.5130 & -1947.8529 & 3.54 & 0.003 & -6.720 & -7.947 & 0.332 & 0.083 & ${ }^{5} \mathrm{H}_{4}$ \\
\hline-1948.5166 & -1947.8568 & 1.09 & 0.160 & -6.702 & -7.953 & 0.315 & 0.091 & ${ }^{5} \mathrm{H}_{4}$ \\
\hline-1948.5162 & -1947.8564 & 1.35 & 0.102 & -6.788 & -7.975 & 0.315 & 0.095 & ${ }^{5} \mathrm{H}_{4}$ \\
\hline-1948.5153 & -1947.8549 & 2.28 & 0.021 & -6.733 & -7.954 & 0.316 & 0.091 & ${ }^{5} \mathrm{H}_{4}$ \\
\hline-1948.5153 & -1947.8553 & 2.04 & 0.032 & -6.695 & -7.970 & 0.316 & 0.089 & ${ }^{5} \mathrm{H}_{4}$ \\
\hline-1948.5165 & -1947.8566 & 1.20 & 0.131 & -6.725 & -7.956 & 0.314 & 0.091 & ${ }^{5} \mathrm{H}_{4}$ \\
\hline-1948.5162 & -1947.8571 & 0.90 & 0.219 & -6.724 & -7.943 & 0.325 & 0.086 & ${ }^{5} \mathrm{H}_{4}$ \\
\hline-1948.5130 & -1947.8540 & 2.84 & 0.008 & -6.762 & -8.045 & 0.316 & 0.100 & ${ }^{5} \mathrm{H}_{4}$ \\
\hline-1948.5138 & -1947.8559 & 1.70 & 0.057 & -6.675 & -8.090 & 0.168 & 0.160 & ${ }^{4} \mathrm{H}_{5}$ \\
\hline-1948.5109 & -1947.8537 & 3.02 & 0.006 & -6.738 & -7.942 & 0.340 & 0.080 & ${ }^{5} \mathrm{H}_{4}$ \\
\hline-1948.5155 & -1947.8564 & 1.33 & 0.106 & -6.727 & -7.977 & 0.313 & 0.095 & ${ }^{5} \mathrm{H}_{4}$ \\
\hline-1948.5165 & -1947.8574 & 0.75 & 0.280 & -6.797 & -7.966 & 0.325 & 0.088 & ${ }^{5} \mathrm{H}_{4}$ \\
\hline-1948.5176 & -1947.8580 & 0.34 & 0.562 & -6.791 & -7.999 & 0.299 & 0.101 & ${ }^{5} \mathrm{H}_{4}$ \\
\hline-1948.5129 & -1947.8536 & 3.13 & 0.005 & -6.748 & -8.021 & 0.323 & 0.098 & ${ }^{5} \mathrm{H}_{4}$ \\
\hline-1948.5150 & -1947.8550 & 2.21 & 0.024 & -6.734 & -8.063 & 0.297 & 0.109 & ${ }^{5} \mathrm{H}_{4}$ \\
\hline-1948.5138 & -1947.8544 & 2.59 & 0.013 & -6.731 & -8.061 & 0.297 & 0.109 & ${ }^{5} \mathrm{H}_{4}$ \\
\hline-1948.5161 & -1947.8562 & 1.50 & 0.080 & -6.782 & -7.989 & 0.311 & 0.099 & ${ }^{5} \mathrm{H}_{4}$ \\
\hline-1948.5165 & -1947.8567 & 1.16 & 0.141 & -6.719 & -7.953 & 0.314 & 0.092 & ${ }^{5} \mathrm{H}_{4}$ \\
\hline-1948.5160 & -1947.8566 & 1.23 & 0.126 & -6.746 & -8.082 & 0.292 & 0.113 & ${ }^{5} \mathrm{H}_{4}$ \\
\hline-1948.5176 & -1947.8578 & 0.49 & 0.436 & -6.783 & -7.983 & 0.296 & 0.100 & ${ }^{5} \mathrm{H}_{4}$ \\
\hline-1948.5177 & -1947.8580 & 0.35 & 0.555 & -6.793 & -7.997 & 0.300 & 0.101 & ${ }^{5} \mathrm{H}_{4}$ \\
\hline-1948.5170 & -1947.8577 & 0.56 & 0.387 & -6.794 & -7.984 & 0.293 & 0.099 & ${ }^{5} \mathrm{H}_{4}$ \\
\hline-1948.5182 & -1947.8586 & 0.00 & 1.000 & -6.791 & -7.990 & 0.297 & 0.100 & ${ }^{5} \mathrm{H}_{4}$ \\
\hline-1948.5152 & -1947.8565 & 1.26 & 0.118 & -6.727 & -8.024 & 0.304 & 0.101 & ${ }^{5} \mathrm{H}_{4}$ \\
\hline-1948.5142 & -1947.8546 & 2.47 & 0.015 & -6.715 & -8.102 & 0.282 & 0.120 & ${ }^{5} \mathrm{H}_{4}$ \\
\hline-1948.5153 & -1947.8561 & 1.56 & 0.071 & -6.711 & -8.048 & 0.305 & 0.104 & ${ }^{5} \mathrm{H}_{4}$ \\
\hline-1948.5151 & -1947.8554 & 1.99 & 0.035 & -6.724 & -8.104 & 0.280 & 0.121 & ${ }^{5} \mathrm{H}_{4}$ \\
\hline-1948.5161 & -1947.8570 & 1.00 & 0.184 & -6.724 & -8.056 & 0.305 & 0.105 & ${ }^{5} \mathrm{H}_{4}$ \\
\hline-1948.5147 & -1947.8539 & 2.90 & 0.007 & -6.700 & -7.954 & 0.317 & 0.090 & ${ }^{5} \mathrm{H}_{4}$ \\
\hline-1948.5152 & -1947.8565 & 1.28 & 0.116 & -6.739 & -8.053 & 0.297 & 0.109 & ${ }^{5} \mathrm{H}_{4}$ \\
\hline-1948.5149 & -1947.8573 & 0.82 & 0.252 & -6.706 & -8.038 & 0.306 & 0.102 & ${ }^{5} \mathrm{H}_{4}$ \\
\hline-1948.5139 & -1947.8543 & 2.69 & 0.011 & -6.716 & -8.106 & 0.282 & 0.120 & ${ }^{5} \mathrm{H}_{4}$ \\
\hline-1948.5135 & -1947.8537 & 3.02 & 0.006 & -6.725 & -8.062 & 0.296 & 0.110 & ${ }^{5} \mathrm{H}_{4}$ \\
\hline-1948.5135 & -1947.8544 & 2.60 & 0.012 & -6.768 & -8.008 & 0.320 & 0.094 & ${ }^{5} \mathrm{H}_{4}$ \\
\hline
\end{tabular}

Table SCY11. Energetics for 12 at the PBE0/D3BJ/CPCM $\left(\mathrm{CH}_{3} \mathrm{CN}\right) / / \mathrm{PBE} 0 / \mathrm{D} 3 \mathrm{BJ} / \mathrm{DEF} 2-$ SVP/RIJCOSX level. Relative energies in $\mathrm{kcal} / \mathrm{mol}$, orbital energies in $\mathrm{eV}$.

\begin{tabular}{|c|c|c|c|c|c|c|c|c|}
\hline E (a.u.) & G (a.u.) & $\Delta \mathrm{G}$ & Boltz. & HOMO & $\begin{array}{c}\pi_{C_{1}-C_{2}} \\
(\mathrm{ev})\end{array}$ & $\begin{array}{c}\Delta E^{(2)}(e V) \\
\left(\pi \rightarrow \sigma_{C_{3}-o}^{*}\right)\end{array}$ & $\begin{array}{c}\Delta E^{(2)}(e V) \\
\left(\pi \rightarrow \sigma_{C_{3}-H}^{*}\right)\end{array}$ & Conformer \\
\hline-1719.6143 & -1719.0161 & 1.77 & 0.050 & -6.644 & -7.985 & 0.330 & 0.088 & ${ }^{5} \mathrm{H}_{4}$ \\
\hline-1719.6105 & -1719.0146 & 2.71 & 0.010 & -6.649 & -8.099 & 0.188 & 0.161 & ${ }^{4} \mathrm{H}_{5}$ \\
\hline-1719.6112 & -1719.0127 & 3.93 & 0.001 & -6.633 & -7.967 & 0.327 & 0.091 & ${ }^{5} \mathrm{H}_{4}$ \\
\hline-1719.6096 & -1719.0122 & 4.26 & 0.001 & -6.660 & -8.165 & 0.191 & 0.158 & ${ }^{4} \mathrm{H}_{5}$ \\
\hline-1719.6131 & -1719.0160 & 1.86 & 0.043 & -6.739 & -8.052 & 0.304 & 0.104 & ${ }^{5} \mathrm{H}_{4}$ \\
\hline-1719.6153 & -1719.0172 & 1.10 & 0.156 & -6.742 & -8.020 & 0.310 & 0.096 & ${ }^{5} \mathrm{H}_{4}$ \\
\hline-1719.6127 & -1719.0143 & 2.92 & 0.007 & -6.729 & -8.046 & 0.304 & 0.104 & ${ }^{5} \mathrm{H}_{4}$ \\
\hline-1719.6162 & -1719.0190 & 0.00 & 1.000 & -6.748 & -8.041 & 0.307 & 0.096 & ${ }^{5} \mathrm{H}_{4}$ \\
\hline-1719.6123 & -1719.0143 & 2.91 & 0.007 & -6.736 & -8.063 & 0.301 & 0.107 & ${ }^{5} \mathrm{H}_{4}$ \\
\hline-1719.6137 & -1719.0155 & 2.18 & 0.025 & -6.758 & -7.998 & 0.027 & 0.217 & ${ }^{4} \mathrm{H}_{5}$ \\
\hline-1719.6139 & -1719.0148 & 2.64 & 0.012 & -6.743 & -8.050 & 0.308 & 0.101 & ${ }^{5} \mathrm{H}_{4}$ \\
\hline-1719.6130 & -1719.0143 & 2.91 & 0.007 & -6.719 & -8.053 & 0.314 & 0.099 & ${ }^{5} \mathrm{H}_{4}$ \\
\hline-1719.6108 & -1719.0125 & 4.04 & 0.001 & -6.734 & -8.073 & 0.299 & 0.109 & ${ }^{5} \mathrm{H}_{4}$ \\
\hline
\end{tabular}




\begin{tabular}{|c|c|c|c|c|c|c|c|c|}
\hline-1719.6152 & -1719.0182 & 0.47 & 0.455 & -6.721 & -8.021 & 0.308 & 0.094 & ${ }^{5} \mathrm{H}_{4}$ \\
\hline-1719.6137 & -1719.0149 & 2.55 & 0.013 & -6.635 & -7.977 & 0.323 & 0.091 & ${ }^{5} \mathrm{H}_{4}$ \\
\hline-1719.6135 & -1719.0156 & 2.13 & 0.028 & -6.699 & -8.016 & 0.343 & 0.084 & ${ }^{5} \mathrm{H}_{4}$ \\
\hline-1719.6143 & -1719.0168 & 1.36 & 0.101 & -6.703 & -8.031 & 0.313 & 0.094 & ${ }^{5} \mathrm{H}_{4}$ \\
\hline-1719.6133 & -1719.0148 & 2.61 & 0.012 & -6.726 & -8.058 & 0.317 & 0.100 & ${ }^{5} \mathrm{H}_{4}$ \\
\hline-1719.6129 & -1719.0141 & 3.05 & 0.006 & -6.642 & -7.982 & 0.322 & 0.092 & ${ }^{5} \mathrm{H}_{4}$ \\
\hline-1719.6104 & -1719.0123 & 4.16 & 0.001 & -6.671 & -8.091 & 0.027 & 0.217 & ${ }^{4} \mathrm{H}_{5}$ \\
\hline-1719.6121 & -1719.0143 & 2.94 & 0.007 & -6.563 & -8.112 & 0.176 & 0.161 & ${ }^{4} \mathrm{H}_{5}$ \\
\hline-1719.6097 & -1719.0122 & 4.23 & 0.001 & -6.658 & -8.165 & 0.189 & 0.159 & ${ }^{4} \mathrm{H}_{5}$ \\
\hline-1719.6126 & -1719.0135 & 3.41 & 0.003 & -6.736 & -8.027 & 0.310 & 0.097 & ${ }^{5} \mathrm{H}_{4}$ \\
\hline-1719.6123 & -1719.0136 & 3.37 & 0.003 & -6.729 & -8.079 & 0.294 & 0.111 & ${ }^{5} \mathrm{H}_{4}$ \\
\hline-1719.6141 & -1719.0165 & 1.55 & 0.073 & -6.741 & -8.055 & 0.303 & 0.105 & ${ }^{5} \mathrm{H}_{4}$ \\
\hline-1719.6133 & -1719.0162 & 1.75 & 0.052 & -6.736 & -8.040 & 0.316 & 0.096 & ${ }^{5} \mathrm{H}_{4}$ \\
\hline-1719.6149 & -1719.0169 & 1.26 & 0.119 & -6.720 & -8.005 & 0.309 & 0.090 & ${ }^{5} \mathrm{H}_{4}$ \\
\hline-1719.6144 & -1719.0157 & 2.05 & 0.032 & -6.720 & -8.019 & 0.304 & 0.101 & ${ }^{5} \mathrm{H}_{4}$ \\
\hline-1719.6121 & -1719.0140 & 3.09 & 0.005 & -6.727 & -8.021 & 0.319 & 0.094 & ${ }^{5} \mathrm{H}_{4}$ \\
\hline-1719.6129 & -1719.0148 & 2.62 & 0.012 & -6.728 & -8.033 & 0.307 & 0.098 & ${ }^{5} \mathrm{H}_{4}$ \\
\hline-1719.6130 & -1719.0143 & 2.93 & 0.007 & -6.736 & -8.064 & 0.299 & 0.108 & ${ }^{5} \mathrm{H}_{4}$ \\
\hline-1719.6104 & -1719.0132 & 3.63 & 0.002 & -6.652 & -8.048 & 0.319 & 0.099 & ${ }^{5} \mathrm{H}_{4}$ \\
\hline-1719.6133 & -1719.0155 & 2.14 & 0.027 & -6.738 & -8.080 & 0.295 & 0.112 & ${ }^{5} \mathrm{H}_{4}$ \\
\hline-1719.6121 & -1719.0147 & 2.65 & 0.011 & -6.730 & -8.032 & 0.319 & 0.095 & ${ }^{5} \mathrm{H}_{4}$ \\
\hline-1719.6134 & -1719.0142 & 2.96 & 0.007 & -6.698 & -8.017 & 0.343 & 0.084 & ${ }^{5} \mathrm{H}_{4}$ \\
\hline-1719.6127 & -1719.0141 & 3.03 & 0.006 & -6.717 & -8.027 & 0.313 & 0.093 & ${ }^{5} \mathrm{H}_{4}$ \\
\hline-1719.6136 & -1719.0151 & 2.43 & 0.016 & -6.619 & -7.989 & 0.320 & 0.092 & ${ }^{5} \mathrm{H}_{4}$ \\
\hline-1719.6104 & -1719.0145 & 2.83 & 0.008 & -6.576 & -8.155 & 0.167 & 0.163 & ${ }^{4} \mathrm{H}_{5}$ \\
\hline-1719.6083 & -1719.0115 & 4.65 & 0.000 & -6.691 & -8.104 & 0.201 & 0.163 & ${ }^{4} \mathrm{H}_{5}$ \\
\hline-1719.6091 & -1719.0132 & 3.59 & 0.002 & -6.662 & -8.156 & 0.183 & 0.162 & ${ }^{4} \mathrm{H}_{5}$ \\
\hline-1719.6132 & -1719.0152 & 2.35 & 0.019 & -6.758 & -8.017 & 0.299 & 0.103 & ${ }^{5} \mathrm{H}_{4}$ \\
\hline-1719.6129 & -1719.0149 & 2.53 & 0.014 & -6.718 & -8.041 & 0.346 & 0.086 & ${ }^{5} \mathrm{H}_{4}$ \\
\hline-1719.6111 & -1719.0140 & 3.13 & 0.005 & -6.645 & -8.139 & 0.173 & 0.163 & ${ }^{4} \mathrm{H}_{5}$ \\
\hline-1719.6138 & -1719.0154 & 2.20 & 0.024 & -6.718 & -8.030 & 0.308 & 0.098 & ${ }^{5} \mathrm{H}_{4}$ \\
\hline-1719.6156 & -1719.0176 & 0.85 & 0.236 & -6.739 & -8.023 & 0.304 & 0.102 & ${ }^{5} \mathrm{H}_{4}$ \\
\hline-1719.6129 & -1719.0149 & 2.53 & 0.014 & -6.733 & -8.086 & 0.291 & 0.115 & ${ }^{5} \mathrm{H}_{4}$ \\
\hline-1719.6150 & -1719.0173 & 1.05 & 0.170 & -6.715 & -8.010 & 0.308 & 0.091 & ${ }^{5} \mathrm{H}_{4}$ \\
\hline-1719.6108 & -1719.0133 & 3.57 & 0.002 & -6.615 & -8.111 & 0.152 & 0.170 & ${ }^{4} \mathrm{H}_{5}$ \\
\hline-1719.6141 & -1719.0163 & 1.65 & 0.062 & -6.730 & -8.053 & 0.301 & 0.106 & ${ }^{5} \mathrm{H}_{4}$ \\
\hline-1719.6133 & -1719.0165 & 1.57 & 0.071 & -6.717 & -8.066 & 0.312 & 0.103 & ${ }^{5} \mathrm{H}_{4}$ \\
\hline-1719.6131 & -1719.0146 & 2.73 & 0.010 & -6.720 & -8.062 & 0.316 & 0.102 & ${ }^{5} \mathrm{H}_{4}$ \\
\hline-1719.6148 & -1719.0159 & 1.90 & 0.040 & -6.698 & -8.010 & 0.307 & 0.085 & ${ }^{5} \mathrm{H}_{4}$ \\
\hline-1719.6112 & -1719.0122 & 4.23 & 0.001 & -6.715 & -8.060 & 0.313 & 0.101 & ${ }^{5} \mathrm{H}_{4}$ \\
\hline-1719.6132 & -1719.0148 & 2.60 & 0.012 & -6.711 & -8.069 & 0.310 & 0.106 & ${ }^{5} \mathrm{H}_{4}$ \\
\hline-1719.6142 & -1719.0155 & 2.18 & 0.025 & -6.648 & -7.988 & 0.319 & 0.093 & ${ }^{5} \mathrm{H}_{4}$ \\
\hline-1719.6090 & -1719.0130 & 3.72 & 0.002 & -6.700 & -8.126 & 0.193 & 0.166 & ${ }^{4} \mathrm{H}_{5}$ \\
\hline-1719.6090 & -1719.0130 & 3.74 & 0.002 & -6.697 & -8.121 & 0.195 & 0.165 & ${ }^{4} \mathrm{H}_{5}$ \\
\hline-1719.6111 & -1719.0154 & 2.22 & 0.024 & -6.648 & -8.156 & 0.185 & 0.162 & ${ }^{4} \mathrm{H}_{5}$ \\
\hline-1719.6123 & -1719.0139 & 3.18 & 0.005 & -6.735 & -8.073 & 0.297 & 0.110 & ${ }^{5} \mathrm{H}_{4}$ \\
\hline-1719.6112 & -1719.0138 & 3.24 & 0.004 & -6.733 & -7.994 & 0.344 & 0.075 & ${ }^{5} \mathrm{H}_{4}$ \\
\hline-1719.6113 & -1719.0133 & 3.53 & 0.003 & -6.728 & -8.051 & 0.302 & 0.103 & ${ }^{5} \mathrm{H}_{4}$ \\
\hline-1719.6137 & -1719.0154 & 2.22 & 0.024 & -6.761 & -8.003 & 0.027 & 0.217 & ${ }^{4} \mathrm{H}_{5}$ \\
\hline-1719.6109 & -1719.0119 & 4.40 & 0.001 & -6.710 & -8.058 & 0.314 & 0.101 & ${ }^{5} \mathrm{H}_{4}$ \\
\hline-1719.6132 & -1719.0136 & 3.37 & 0.003 & -6.739 & -8.049 & 0.309 & 0.101 & ${ }^{5} \mathrm{H}_{4}$ \\
\hline-1719.6128 & -1719.0149 & 2.57 & 0.013 & -6.736 & -8.026 & 0.349 & 0.081 & ${ }^{5} \mathrm{H}_{4}$ \\
\hline-1719.6122 & -1719.0145 & 2.83 & 0.008 & -6.596 & -8.120 & 0.151 & 0.170 & ${ }^{4} \mathrm{H}_{5}$ \\
\hline-1719.6132 & -1719.0156 & 2.11 & 0.029 & -6.742 & -8.004 & 0.345 & 0.081 & ${ }^{5} \mathrm{H}_{4}$ \\
\hline-1719.6137 & -1719.0156 & 2.10 & 0.029 & -6.729 & -8.051 & 0.317 & 0.098 & ${ }^{5} \mathrm{H}_{4}$ \\
\hline-1719.6148 & -1719.0175 & 0.94 & 0.206 & -6.720 & -8.025 & 0.312 & 0.091 & ${ }^{5} \mathrm{H}_{4}$ \\
\hline-1719.6128 & -1719.0145 & 2.81 & 0.009 & -6.717 & -8.056 & 0.314 & 0.102 & ${ }^{5} \mathrm{H}_{4}$ \\
\hline-1719.6109 & -1719.0141 & 3.07 & 0.006 & -6.646 & -8.136 & 0.028 & 0.217 & ${ }^{4} \mathrm{H}_{5}$ \\
\hline-1719.6145 & -1719.0158 & 1.97 & 0.036 & -6.636 & -7.977 & 0.318 & 0.092 & ${ }^{5} \mathrm{H}_{4}$ \\
\hline-1719.6109 & -1719.0128 & 3.89 & 0.001 & -6.648 & -7.956 & 0.343 & 0.080 & ${ }^{5} \mathrm{H}_{4}$ \\
\hline-1719.6104 & -1719.0135 & 3.45 & 0.003 & -6.651 & -8.046 & 0.319 & 0.098 & ${ }^{5} \mathrm{H}_{4}$ \\
\hline-1719.6109 & -1719.0141 & 3.07 & 0.006 & -6.578 & -8.138 & 0.152 & 0.161 & ${ }^{4} \mathrm{H}_{5}$ \\
\hline
\end{tabular}




\begin{tabular}{|c|c|c|c|c|c|c|c|c|}
\hline-1719.6096 & -1719.0123 & 4.20 & 0.001 & -6.662 & -8.169 & 0.190 & 0.158 & ${ }^{4} \mathrm{H}_{5}$ \\
\hline-1719.6126 & -1719.0146 & 2.75 & 0.010 & -6.748 & -8.001 & 0.027 & 0.217 & ${ }^{4} \mathrm{H}_{5}$ \\
\hline-1719.6101 & -1719.0119 & 4.42 & 0.001 & -6.728 & -8.067 & 0.299 & 0.108 & ${ }^{5} \mathrm{H}_{4}$ \\
\hline-1719.6136 & -1719.0156 & 2.11 & 0.028 & -6.730 & -7.978 & 0.343 & 0.072 & ${ }^{5} \mathrm{H}_{4}$ \\
\hline-1719.6140 & -1719.0163 & 1.65 & 0.062 & -6.735 & -8.056 & 0.303 & 0.105 & ${ }^{5} \mathrm{H}_{4}$ \\
\hline-1719.6103 & -1719.0133 & 3.54 & 0.003 & -6.665 & -8.040 & 0.316 & 0.100 & ${ }^{5} \mathrm{H}_{4}$ \\
\hline-1719.6128 & -1719.0142 & 2.99 & 0.006 & -6.732 & -8.093 & 0.289 & 0.116 & ${ }^{5} \mathrm{H}_{4}$ \\
\hline-1719.6130 & -1719.0141 & 3.02 & 0.006 & -6.662 & -7.974 & 0.321 & 0.091 & ${ }^{5} \mathrm{H}_{4}$ \\
\hline-1719.6141 & -1719.0152 & 2.34 & 0.019 & -6.649 & -8.127 & 0.028 & 0.217 & ${ }^{4} \mathrm{H}_{5}$ \\
\hline-1719.6120 & -1719.0141 & 3.06 & 0.006 & -6.727 & -8.027 & 0.322 & 0.095 & ${ }^{5} \mathrm{H}_{4}$ \\
\hline-1719.6134 & -1719.0140 & 3.11 & 0.005 & -6.635 & -7.976 & 0.320 & 0.091 & ${ }^{5} \mathrm{H}_{4}$ \\
\hline-1719.6103 & -1719.0112 & 4.86 & 0.000 & -6.628 & -7.965 & 0.327 & 0.090 & ${ }^{5} \mathrm{H}_{4}$ \\
\hline-1719.6135 & -1719.0145 & 2.79 & 0.009 & -6.645 & -7.966 & 0.319 & 0.090 & ${ }^{5} \mathrm{H}_{4}$ \\
\hline-1719.6147 & -1719.0160 & 1.83 & 0.045 & -6.741 & -8.068 & 0.300 & 0.108 & ${ }^{5} \mathrm{H}_{4}$ \\
\hline-1719.6146 & -1719.0158 & 1.98 & 0.035 & -6.637 & -7.980 & 0.318 & 0.092 & ${ }^{5} \mathrm{H}_{4}$ \\
\hline-1719.6142 & -1719.0161 & 1.77 & 0.051 & -6.635 & -7.980 & 0.330 & 0.088 & ${ }^{5} \mathrm{H}_{4}$ \\
\hline-1719.6112 & -1719.0127 & 3.93 & 0.001 & -6.628 & -7.961 & 0.326 & 0.091 & ${ }^{5} \mathrm{H}_{4}$ \\
\hline-1719.6087 & -1719.0112 & 4.85 & 0.000 & -6.579 & -8.107 & 0.175 & 0.163 & ${ }^{4} \mathrm{H}_{5}$ \\
\hline-1719.6138 & -1719.0153 & 2.32 & 0.020 & -6.716 & -8.028 & 0.310 & 0.094 & ${ }^{5} \mathrm{H}_{4}$ \\
\hline-1719.6156 & -1719.0176 & 0.87 & 0.232 & -6.740 & -8.021 & 0.304 & 0.102 & ${ }^{5} \mathrm{H}_{4}$ \\
\hline-1719.6149 & -1719.0161 & 1.76 & 0.051 & -6.747 & -8.012 & 0.303 & 0.103 & ${ }^{5} \mathrm{H}_{4}$ \\
\hline-1719.6141 & -1719.0155 & 2.19 & 0.025 & -6.731 & -8.042 & 0.307 & 0.101 & ${ }^{5} \mathrm{H}_{4}$ \\
\hline-1719.6147 & -1719.0159 & 1.93 & 0.038 & -6.752 & -8.066 & 0.301 & 0.106 & ${ }^{5} \mathrm{H}_{4}$ \\
\hline-1719.6103 & -1719.0133 & 3.53 & 0.003 & -6.664 & -8.034 & 0.317 & 0.099 & ${ }^{5} \mathrm{H}_{4}$ \\
\hline-1719.6140 & -1719.0168 & 1.38 & 0.098 & -6.720 & -8.010 & 0.303 & 0.093 & ${ }^{5} \mathrm{H}_{4}$ \\
\hline-1719.6124 & -1719.0144 & 2.88 & 0.008 & -6.652 & -7.961 & 0.330 & 0.086 & ${ }^{5} \mathrm{H}_{4}$ \\
\hline-1719.6117 & -1719.0138 & 3.23 & 0.004 & -6.639 & -8.141 & 0.028 & 0.217 & ${ }^{4} \mathrm{H}_{5}$ \\
\hline-1719.6139 & -1719.0167 & 1.43 & 0.090 & -6.730 & -8.045 & 0.303 & 0.104 & ${ }^{5} \mathrm{H}_{4}$ \\
\hline-1719.6127 & -1719.0143 & 2.93 & 0.007 & -6.659 & -7.993 & 0.334 & 0.088 & ${ }^{5} \mathrm{H}_{4}$ \\
\hline-1719.6128 & -1719.0156 & 2.10 & 0.029 & -6.722 & -8.019 & 0.315 & 0.092 & ${ }^{5} \mathrm{H}_{4}$ \\
\hline-1719.6113 & -1719.0129 & 3.82 & 0.002 & -6.638 & -7.973 & 0.326 & 0.092 & ${ }^{5} \mathrm{H}_{4}$ \\
\hline-1719.6128 & -1719.0145 & 2.78 & 0.009 & -6.730 & -8.058 & 0.319 & 0.103 & ${ }^{5} \mathrm{H}_{4}$ \\
\hline
\end{tabular}

Table SCY12. Energetics for 13 at the PBE0/D3BJ/CPCM $\left(\mathrm{CH}_{3} \mathrm{CN}\right) / / \mathrm{PBE} 0 / \mathrm{D} 3 \mathrm{BJ} / \mathrm{DEF} 2-$ $\mathrm{SVP} / \mathrm{RIJCOSX}$ level. Relative energies in $\mathrm{kcal} / \mathrm{mol}$, orbital energies in $\mathrm{eV}$.

\begin{tabular}{|c|c|c|c|c|c|c|c|c|}
\hline E (a.u.) & G (a.u.) & $\Delta \mathrm{G}$ & Boltz. & HOMO & $\begin{array}{c}\pi_{C_{1}-C_{2}} \\
\quad(\mathrm{ev})\end{array}$ & $\begin{array}{c}\Delta E^{(2)}(e V) \\
\left(\pi \rightarrow \sigma_{C_{3}-o}^{*}\right)\end{array}$ & $\begin{array}{c}\Delta E^{(2)}(e V) \\
\left(\pi \rightarrow \sigma_{C_{3}-H}^{*}\right)\end{array}$ & Conformer \\
\hline-1227.9941 & -1227.6213 & 1.54 & 0.074 & -6.825 & -8.057 & 0.321 & 0.094 & ${ }^{5} \mathrm{H}_{4}$ \\
\hline-1227.9961 & -1227.6225 & 0.78 & 0.268 & -6.846 & -8.045 & 0.301 & 0.101 & ${ }^{5} \mathrm{H}_{4}$ \\
\hline-1227.9939 & -1227.6192 & 2.87 & 0.008 & -6.856 & -8.120 & 0.303 & 0.105 & ${ }^{5} \mathrm{H}_{4}$ \\
\hline-1227.9965 & -1227.6231 & 0.42 & 0.495 & -6.831 & -8.045 & 0.301 & 0.101 & ${ }^{5} \mathrm{H}_{4}$ \\
\hline-1227.9970 & -1227.6228 & 0.62 & 0.351 & -6.886 & -8.100 & 0.309 & 0.100 & ${ }^{5} \mathrm{H}_{4}$ \\
\hline-1227.9953 & -1227.6213 & 1.52 & 0.077 & -6.813 & -8.055 & 0.311 & 0.088 & ${ }^{5} \mathrm{H}_{4}$ \\
\hline-1227.9943 & -1227.6218 & 1.20 & 0.131 & -6.712 & -8.136 & 0.186 & 0.162 & ${ }^{4} \mathrm{H}_{5}$ \\
\hline-1227.9927 & -1227.6188 & 3.08 & 0.006 & -6.739 & -8.139 & 0.170 & 0.167 & ${ }^{4} \mathrm{H}_{5}$ \\
\hline-1227.9955 & -1227.6222 & 0.97 & 0.195 & -6.741 & -8.152 & 0.194 & 0.164 & ${ }^{4} \mathrm{H}_{5}$ \\
\hline-1227.9940 & -1227.6209 & 1.78 & 0.049 & -6.727 & -8.011 & 0.352 & 0.078 & ${ }^{5} \mathrm{H}_{4}$ \\
\hline-1227.9955 & -1227.6201 & 2.29 & 0.021 & -6.709 & -8.047 & 0.305 & 0.096 & ${ }^{5} \mathrm{H}_{4}$ \\
\hline-1227.9965 & -1227.6213 & 1.51 & 0.078 & -6.741 & -8.028 & 0.316 & 0.093 & ${ }^{5} \mathrm{H}_{4}$ \\
\hline-1227.9943 & -1227.6197 & 2.51 & 0.014 & -6.858 & -8.117 & 0.309 & 0.100 & ${ }^{5} \mathrm{H}_{4}$ \\
\hline-1227.9923 & -1227.6216 & 1.38 & 0.098 & -6.715 & -8.170 & 0.173 & 0.169 & ${ }^{4} \mathrm{H}_{5}$ \\
\hline-1227.9961 & -1227.6233 & 0.29 & 0.612 & -6.692 & -8.178 & 0.172 & 0.163 & ${ }^{4} \mathrm{H}_{5}$ \\
\hline-1227.9939 & -1227.6218 & 1.22 & 0.127 & -6.807 & -8.065 & 0.350 & 0.079 & ${ }^{5} \mathrm{H}_{4}$ \\
\hline-1227.9963 & -1227.6211 & 1.63 & 0.063 & -6.736 & -8.023 & 0.317 & 0.093 & ${ }^{5} \mathrm{H}_{4}$ \\
\hline-1227.9938 & -1227.6207 & 1.94 & 0.038 & -6.855 & -8.045 & 0.361 & 0.075 & ${ }^{5} \mathrm{H}_{4}$ \\
\hline-1227.9930 & -1227.6207 & 1.89 & 0.041 & -6.696 & -8.166 & 0.174 & 0.162 & ${ }^{4} \mathrm{H}_{5}$ \\
\hline-1227.9952 & -1227.6237 & 0.00 & 1.000 & -6.680 & -8.160 & 0.178 & 0.162 & ${ }^{4} \mathrm{H}_{5}$ \\
\hline-1227.9900 & -1227.6183 & 3.39 & 0.003 & -6.588 & -8.231 & 0.150 & 0.167 & ${ }^{4} \mathrm{H}_{5}$ \\
\hline-1227.9940 & -1227.6215 & 1.43 & 0.089 & -6.705 & -8.138 & 0.184 & 0.162 & ${ }^{4} \mathrm{H}_{5}$ \\
\hline-1227.9938 & -1227.6216 & 1.37 & 0.099 & -6.712 & -8.129 & 0.193 & 0.167 & ${ }^{4} \mathrm{H}_{5}$ \\
\hline
\end{tabular}




\begin{tabular}{|c|c|c|c|c|c|c|c|}
\hline-1227.9944 & -1227.6220 & 1.09 & 0.160 & -6.712 & -8.137 & 0.186 & 0.162 \\
\hline-1227.9960 & -1227.6220 & 1.09 & 0.159 & -6.831 & -8.048 & 0.301 & 0.101 \\
\hline-1227.9940 & -1227.6205 & 2.01 & 0.034 & -6.737 & -7.997 & 0.323 & 0.090 \\
\hline-1227.9965 & -1227.6229 & 0.53 & 0.409 & -6.834 & -8.048 & 0.301 & 0.101 \\
\hline-1227.9909 & -1227.6215 & 1.41 & 0.092 & -6.776 & -8.181 & 0.181 & 0.167 \\
\hline-1227.9902 & -1227.6179 & 3.67 & 0.002 & -6.712 & -8.186 & 0.172 & 0.168 \\
\hline-1227.9953 & -1227.6227 & 0.65 & 0.333 & -6.738 & -8.132 & 0.194 & 0.165 \\
\hline-1227.9924 & -1227.6202 & 2.19 & 0.025 & -6.627 & -8.212 & 0.159 & 0.165 \\
\hline-1227.9935 & -1227.6191 & 2.89 & 0.008 & -6.679 & -8.153 & 0.176 & 0.163 \\
\hline-1227.9946 & -1227.6202 & 2.25 & 0.022 & -6.788 & -8.019 & 0.308 & 0.096 \\
\hline-1227.9939 & -1227.6193 & 2.79 & 0.009 & -6.856 & -8.117 & 0.303 & 0.105 \\
\hline-1227.9914 & -1227.6190 & 2.96 & 0.007 & -6.860 & -8.058 & 0.338 & 0.081 \\
\hline-1227.9923 & -1227.6195 & 2.69 & 0.011 & -6.733 & -8.156 & 0.180 & 0.162 \\
\hline-1227.9900 & -1227.6177 & 3.77 & 0.002 & -6.710 & -8.184 & 0.173 & 0.168 \\
\hline-1227.9911 & -1227.6179 & 3.67 & 0.002 & -6.721 & -8.160 & 0.182 & 0.162 \\
\hline-1227.9911 & -1227.6202 & 2.20 & 0.024 & -6.748 & -8.146 & 0.195 & 0.165 \\
\hline-1227.9883 & -1227.6169 & 4.27 & 0.001 & -6.704 & -8.192 & 0.175 & 0.168 \\
\hline-1227.9897 & -1227.6171 & 4.19 & 0.001 & -6.683 & -8.168 & 0.179 & 0.166 \\
\hline-1227.9930 & -1227.6221 & 1.06 & 0.167 & -6.775 & -8.149 & 0.195 & 0.164 \\
\hline-1227.9917 & -1227.6188 & 3.09 & 0.005 & -6.763 & -8.150 & 0.188 & 0.162 \\
\hline-1227.9933 & -1227.6212 & 1.62 & 0.065 & -6.690 & -8.159 & 0.178 & 0.162 \\
\hline-1227.9903 & -1227.6207 & 1.91 & 0.040 & -6.745 & -8.186 & 0.179 & 0.167 \\
\hline-1227.9940 & -1227.6223 & 0.90 & 0.219 & -6.818 & -8.054 & 0.347 & 0.081 \\
\hline-1227.9935 & -1227.6192 & 2.84 & 0.008 & -6.722 & -8.004 & 0.325 & 0.088 \\
\hline-1227.9925 & -1227.6197 & 2.51 & 0.014 & -6.735 & -8.155 & 0.180 & 0.162 \\
\hline-1227.9881 & -1227.6165 & 4.57 & 0.000 & -6.727 & -8.175 & 0.176 & 0.168 \\
\hline-1227.9899 & -1227.6171 & 4.15 & 0.001 & -6.654 & -8.189 & 0.163 & 0.163 \\
\hline-1227.9921 & -1227.6201 & 2.32 & 0.020 & -6.716 & -8.213 & 0.185 & 0.159 \\
\hline-1227.9922 & -1227.6204 & 2.09 & 0.029 & -6.759 & -8.133 & 0.196 & 0.163 \\
\hline-1227.9934 & -1227.6206 & 2.00 & 0.034 & -6.718 & -8.151 & 0.182 & 0.161 \\
\hline-1227.9900 & -1227.6172 & 4.13 & 0.001 & -6.645 & -8.184 & 0.161 & 0.163 \\
\hline-1227.9910 & -1227.6196 & 2.63 & 0.012 & -6.653 & -8.211 & 0.162 & 0.163 \\
\hline-1227.9902 & -1227.6186 & 3.22 & 0.004 & -6.579 & -8.228 & 0.154 & 0.167 \\
\hline-1227.9915 & -1227.6187 & 3.15 & 0.005 & -6.631 & -8.147 & 0.172 & 0.163 \\
\hline-1227.9889 & -1227.6160 & 4.86 & 0.000 & -6.711 & -8.159 & 0.182 & 0.162 \\
\hline-1227.9901 & -1227.6162 & 4.75 & 0.000 & -6.648 & -8.177 & 0.146 & 0.170 \\
\hline-1227.9916 & -1227.6184 & 3.38 & 0.003 & -6.665 & -8.159 & 0.180 & 0.162 \\
\hline-1227.9902 & -1227.6193 & 2.79 & 0.009 & -6.735 & -8.132 & 0.197 & 0.165 \\
\hline-1227.9875 & -1227.6158 & 4.96 & 0.000 & -6.666 & -8.205 & 0.163 & 0.157 \\
\hline-1227.9927 & -1227.6199 & 2.44 & 0.016 & -6.731 & -8.148 & 0.186 & 0.160 \\
\hline-1227.9922 & -1227.6198 & 2.46 & 0.016 & -6.708 & -8.209 & 0.181 & 0.160 \\
\hline-1227.9935 & -1227.6213 & 1.57 & 0.071 & -6.688 & -8.157 & 0.178 & 0.162 \\
\hline-1227.9891 & -1227.6165 & 4.58 & 0.000 & -6.748 & -8.142 & 0.196 & 0.163 \\
\hline-1227.9910 & -1227.6175 & 3.91 & 0.001 & -6.616 & -8.190 & 0.172 & 0.147 \\
\hline-1227.9902 & -1227.6177 & 3.82 & 0.002 & -6.727 & -8.129 & 0.198 & 0.157 \\
\hline
\end{tabular}

Table SCY13. Energetics for 14 at the PBE0/D3BJ/CPCM $\left(\mathrm{CH}_{3} \mathrm{CN}\right) / / \mathrm{PBE} 0 / \mathrm{D} 3 \mathrm{BJ} / \mathrm{DEF} 2-$ SVP/RIJCOSX level. Relative energies in $\mathrm{kcal} / \mathrm{mol}$, orbital energies in $\mathrm{eV}$.

\begin{tabular}{|c|c|c|c|c|c|c|c|c|}
\hline E (a.u.) & G (a.u.) & $\Delta \mathrm{G}$ & Boltz. & HOMO & $\begin{array}{c}\pi_{C_{1}-C_{2}} \\
(\mathrm{ev})\end{array}$ & $\begin{array}{c}\Delta E^{(2)}(e V) \\
\left(\pi \rightarrow \sigma_{C_{3}-O}^{*}\right)\end{array}$ & $\begin{array}{c}\Delta E^{(2)}(e V) \\
\left(\pi \rightarrow \sigma_{C_{3}-H}^{*}\right)\end{array}$ & Conforme \\
\hline-1114.6944 & -1114.3328 & 1.53 & 0.076 & -6.719 & -8.152 & 0.186 & 0.168 & ${ }^{4} \mathrm{H}_{5}$ \\
\hline-1114.7011 & -1114.3352 & 0.00 & 1.000 & -6.778 & -8.013 & 0.304 & 0.102 & ${ }^{5} \mathrm{H}_{4}$ \\
\hline-1114.6988 & -1114.3339 & 0.86 & 0.233 & -6.789 & -8.015 & 0.320 & 0.088 & ${ }^{5} \mathrm{H}_{4}$ \\
\hline-1114.6950 & -1114.3316 & 2.31 & 0.020 & -6.689 & -8.100 & 0.200 & 0.167 & ${ }^{4} \mathrm{H}_{5}$ \\
\hline-1114.6966 & -1114.3325 & 1.71 & 0.055 & -6.699 & -8.031 & 0.320 & 0.095 & ${ }^{5} \mathrm{H}_{4}$ \\
\hline-1114.6976 & -1114.3316 & 2.28 & 0.021 & -6.699 & -7.994 & 0.307 & 0.095 & ${ }^{5} \mathrm{H}_{4}$ \\
\hline-1114.6952 & -1114.3316 & 2.26 & 0.022 & -6.554 & -8.206 & 0.156 & 0.168 & ${ }^{4} \mathrm{H}_{5}$ \\
\hline-1114.6981 & -1114.3319 & 2.08 & 0.030 & -6.673 & -8.006 & 0.301 & 0.101 & ${ }^{5} \mathrm{H}_{4}$ \\
\hline-1114.6988 & -1114.3331 & 1.34 & 0.105 & -6.801 & -8.032 & 0.310 & 0.095 & ${ }^{5} \mathrm{H}_{4}$ \\
\hline-1114.6979 & -1114.3317 & 2.25 & 0.022 & -6.696 & -7.984 & 0.317 & 0.089 & ${ }^{5} \mathrm{H}_{4}$ \\
\hline
\end{tabular}




$\begin{array}{lllllllll}-1114.6972 & -1114.3334 & 1.14 & 0.147 & -6.729 & -8.111 & 0.197 & 0.165 & { }^{4} \mathrm{H}_{5} \\ -1114.6976 & -1114.3339 & 0.82 & 0.250 & -6.592 & -8.146 & 0.158 & 0.164 & { }^{4} \mathrm{H}_{5} \\ -1114.6975 & -1114.3315 & 2.36 & 0.019 & -6.672 & -7.973 & 0.327 & 0.089 & { }^{5} \mathrm{H}_{4} \\ -1114.6973 & -1114.3325 & 1.74 & 0.053 & -6.696 & -8.125 & 0.186 & 0.162 & { }^{4} \mathrm{H}_{5} \\ -1114.6994 & -1114.3351 & 0.08 & 0.880 & -6.616 & -8.122 & 0.180 & 0.163 & { }^{4} \mathrm{H}_{5} \\ -1114.6946 & -1114.3301 & 3.21 & 0.004 & -6.848 & -8.038 & 0.296 & 0.091 & { }^{5} \mathrm{H}_{4} \\ -1114.6985 & -1114.3329 & 1.49 & 0.081 & -6.688 & -8.002 & 0.317 & 0.091 & { }^{5} \mathrm{H}_{4} \\ -1114.6967 & -1114.3322 & 1.91 & 0.040 & -6.701 & -8.034 & 0.320 & 0.095 & { }^{5} \mathrm{H}_{4} \\ -1114.6984 & -1114.3322 & 1.88 & 0.042 & -6.715 & -7.982 & 0.322 & 0.081 & { }^{5} \mathrm{H}_{4} \\ -1114.6983 & -1114.3331 & 1.35 & 0.102 & -6.600 & -8.139 & 0.144 & 0.172 & { }^{4} \mathrm{H}_{5} \\ -1114.6988 & -1114.3327 & 1.57 & 0.070 & -6.762 & -8.045 & 0.310 & 0.086 & { }^{5} \mathrm{H}_{4} \\ -1114.6960 & -1114.3305 & 2.97 & 0.007 & -6.617 & -8.143 & 0.146 & 0.170 & { }^{4} \mathrm{H}_{5} \\ -1114.6985 & -1114.3335 & 1.09 & 0.158 & -6.657 & -8.109 & 0.183 & 0.163 & { }^{4} \mathrm{H}_{5} \\ -1114.6969 & -1114.3318 & 2.16 & 0.026 & -6.667 & -8.114 & 0.188 & 0.160 & { }^{4} \mathrm{H}_{5} \\ -1114.6977 & -1114.3318 & 2.17 & 0.026 & -6.701 & -7.996 & 0.305 & 0.096 & { }^{5} \mathrm{H}_{4} \\ -1114.6973 & -1114.3327 & 1.57 & 0.071 & -6.675 & -8.179 & 0.189 & 0.160 & { }^{4} \mathrm{H}_{5} \\ -1114.6987 & -1114.3325 & 1.71 & 0.056 & -6.670 & -7.999 & 0.307 & 0.098 & { }^{5} \mathrm{H}_{4} \\ -1114.6971 & -1114.3333 & 1.24 & 0.122 & -6.731 & -8.112 & 0.197 & 0.165 & { }^{4} \mathrm{H}_{5} \\ -1114.6983 & -1114.3342 & 0.65 & 0.335 & -6.619 & -8.135 & 0.177 & 0.164 & { }^{4} \mathrm{H}_{5} \\ -1114.6949 & -1114.3312 & 2.54 & 0.014 & -6.835 & -8.023 & 0.302 & 0.089 & { }^{5} \mathrm{H}_{4} \\ -1114.6978 & -1114.3340 & 0.79 & 0.262 & -6.598 & -8.147 & 0.160 & 0.164 & { }^{4} \mathrm{H}_{5}\end{array}$

Table SCY14. Energetics for 15 at the PBE0/D3BJ/CPCM $\left(\mathrm{CH}_{3} \mathrm{CN}\right) / / \mathrm{PBE} 0 / \mathrm{D} 3 \mathrm{BJ} / \mathrm{DEF} 2-$ $\mathrm{SVP} / \mathrm{RIJCOSX}$ level. Relative energies in $\mathrm{kcal} / \mathrm{mol}$, orbital energies in $\mathrm{eV}$.

\begin{tabular}{|c|c|c|c|c|c|c|c|c|}
\hline E (a.u.) & G (a.u.) & $\Delta \mathrm{G}$ & Boltz. & HOMO & $\begin{array}{c}\pi_{C_{1}-C_{2}} \\
\quad(\mathrm{ev})\end{array}$ & $\begin{array}{c}\Delta E^{(2)}(e V) \\
\left(\pi \rightarrow \sigma_{C_{3}-O}^{*}\right)\end{array}$ & $\begin{array}{c}\Delta E^{(2)}(e V) \\
\left(\pi \rightarrow \sigma_{C_{3}-H}^{*}\right)\end{array}$ & Conformer \\
\hline-2720.8451 & -2720.4693 & 3.45 & 0.003 & -6.759 & -8.028 & 0.305 & 0.092 & ${ }^{5} \mathrm{H}_{4}$ \\
\hline-2720.8465 & -2720.4723 & 1.54 & 0.075 & -6.855 & -8.064 & 0.296 & 0.099 & ${ }^{5} \mathrm{H}_{4}$ \\
\hline-2720.8453 & -2720.4701 & 2.91 & 0.007 & -6.807 & -8.050 & 0.301 & 0.095 & ${ }^{5} \mathrm{H}_{4}$ \\
\hline-2720.8460 & -2720.4704 & 2.76 & 0.009 & -6.769 & -8.039 & 0.308 & 0.091 & ${ }^{5} \mathrm{H}_{4}$ \\
\hline-2720.8472 & -2720.4714 & 2.10 & 0.029 & -6.774 & -8.053 & 0.308 & 0.097 & ${ }^{5} \mathrm{H}_{4}$ \\
\hline-2720.8471 & -2720.4723 & 1.55 & 0.073 & -6.867 & -8.068 & 0.299 & 0.098 & ${ }^{5} \mathrm{H}_{4}$ \\
\hline-2720.8478 & -2720.4724 & 1.47 & 0.084 & -6.920 & -8.160 & 0.300 & 0.109 & ${ }^{5} \mathrm{H}_{4}$ \\
\hline-2720.8472 & -2720.4714 & 2.10 & 0.029 & -6.813 & -8.052 & 0.302 & 0.096 & ${ }^{5} \mathrm{H}_{4}$ \\
\hline-2720.8487 & -2720.4748 & 0.00 & 1.000 & -6.930 & -8.133 & 0.304 & 0.103 & ${ }^{5} \mathrm{H}_{4}$ \\
\hline-2720.8470 & -2720.4713 & 2.19 & 0.025 & -6.818 & -8.056 & 0.302 & 0.096 & ${ }^{5} \mathrm{H}_{4}$ \\
\hline-2720.8460 & -2720.4697 & 3.18 & 0.005 & -6.731 & -8.077 & 0.297 & 0.097 & ${ }^{5} \mathrm{H}_{4}$ \\
\hline-2720.8458 & -2720.4703 & 2.80 & 0.009 & -6.737 & -8.068 & 0.301 & 0.094 & ${ }^{5} \mathrm{H}_{4}$ \\
\hline-2720.8472 & -2720.4724 & 1.49 & 0.081 & -6.775 & -8.051 & 0.311 & 0.092 & ${ }^{5} \mathrm{H}_{4}$ \\
\hline-2720.8457 & -2720.4707 & 2.53 & 0.014 & -6.768 & -8.023 & 0.341 & 0.077 & ${ }^{5} \mathrm{H}_{4}$ \\
\hline-2720.8477 & -2720.4724 & 1.50 & 0.079 & -6.758 & -8.060 & 0.316 & 0.089 & ${ }^{5} \mathrm{H}_{4}$ \\
\hline-2720.8480 & -2720.4727 & 1.29 & 0.113 & -6.915 & -8.125 & 0.316 & 0.094 & ${ }^{5} \mathrm{H}_{4}$ \\
\hline-2720.8469 & -2720.4717 & 1.91 & 0.040 & -6.904 & -8.122 & 0.342 & 0.085 & ${ }^{5} \mathrm{H}_{4}$ \\
\hline-2720.8480 & -2720.4727 & 1.30 & 0.112 & -6.916 & -8.124 & 0.316 & 0.094 & ${ }^{5} \mathrm{H}_{4}$ \\
\hline-2720.8465 & -2720.4724 & 1.51 & 0.079 & -6.777 & -8.144 & 0.215 & 0.155 & ${ }^{4} \mathrm{H}_{5}$ \\
\hline-2720.8464 & -2720.4745 & 0.21 & 0.705 & -6.746 & -8.159 & 0.183 & 0.167 & ${ }^{4} \mathrm{H}_{5}$ \\
\hline-2720.8431 & -2720.4709 & 2.47 & 0.015 & -6.774 & -8.167 & 0.186 & 0.165 & ${ }^{4} \mathrm{H}_{5}$ \\
\hline-2720.8441 & -2720.4710 & 2.37 & 0.018 & -6.744 & -8.172 & 0.182 & 0.165 & ${ }^{4} \mathrm{H}_{5}$ \\
\hline-2720.8464 & -2720.4739 & 0.53 & 0.408 & -6.754 & -8.147 & 0.191 & 0.165 & ${ }^{4} \mathrm{H}_{5}$ \\
\hline-2720.8447 & -2720.4726 & 1.37 & 0.099 & -6.730 & -8.152 & 0.186 & 0.167 & ${ }^{4} \mathrm{H}_{5}$ \\
\hline-2720.8465 & -2720.4723 & 1.54 & 0.075 & -6.771 & -8.065 & 0.320 & 0.088 & ${ }^{5} \mathrm{H}_{4}$ \\
\hline-2720.8432 & -2720.4703 & 2.79 & 0.009 & -6.746 & -8.198 & 0.173 & 0.167 & ${ }^{4} \mathrm{H}_{5}$ \\
\hline-2720.8441 & -2720.4714 & 2.11 & 0.029 & -6.770 & -8.244 & 0.173 & 0.166 & ${ }^{4} \mathrm{H}_{5}$ \\
\hline-2720.8449 & -2720.4727 & 1.32 & 0.108 & -6.729 & -8.147 & 0.190 & 0.166 & ${ }^{4} \mathrm{H}_{5}$ \\
\hline-2720.8413 & -2720.4685 & 3.96 & 0.001 & -6.721 & -8.216 & 0.165 & 0.168 & ${ }^{4} \mathrm{H}_{5}$ \\
\hline-2720.8471 & -2720.4743 & 0.29 & 0.614 & -6.705 & -8.188 & 0.172 & 0.163 & ${ }^{4} \mathrm{H}_{5}$ \\
\hline-2720.8413 & -2720.4688 & 3.77 & 0.002 & -6.680 & -8.206 & 0.175 & 0.165 & ${ }^{4} \mathrm{H}_{5}$ \\
\hline-2720.8470 & -2720.4726 & 1.38 & 0.097 & -6.698 & -8.175 & 0.170 & 0.162 & ${ }^{4} \mathrm{H}_{5}$ \\
\hline-2720.8468 & -2720.4742 & 0.35 & 0.554 & -6.703 & -8.189 & 0.171 & 0.163 & ${ }^{4} \mathrm{H}_{5}$ \\
\hline
\end{tabular}




$\begin{array}{lllllllll}-2720.8449 & -2720.4704 & 2.75 & 0.010 & -6.695 & -8.184 & 0.174 & 0.161 & 0.162 \\ -2720.8470 & -2720.4728 & 1.24 & 0.124 & -6.692 & -8.169 & 0.171 & { }^{4} \mathrm{H}_{5} \\ -2720.8428 & -2720.4695 & 3.29 & 0.004 & -6.746 & -8.178 & 0.181 & 0.165 & { }^{4} \mathrm{H}_{5} \\ -2720.8425 & -2720.4697 & 3.19 & 0.005 & -6.756 & -8.166 & 0.186 & 0.158 & { }^{4} \mathrm{H}_{5} \\ -2720.8423 & -2720.4689 & 3.69 & 0.002 & -6.749 & -8.183 & 0.180 & 0.165 & { }^{4} \mathrm{H}_{5} \\ -2720.8393 & -2720.4648 & 6.28 & 0.000 & -6.643 & -8.161 & 0.163 & 0.163 & { }^{4} \mathrm{H}_{5} \\ -2720.8448 & -2720.4709 & 2.43 & 0.016 & -6.702 & -8.214 & 0.170 & 0.160 & { }^{4} \mathrm{H}_{5} \\ -2720.8428 & -2720.4707 & 2.57 & 0.013 & -6.734 & -8.223 & 0.187 & 0.158 & { }^{4} \mathrm{H}_{5} \\ -2720.8410 & -2720.4660 & 5.53 & 0.000 & -6.713 & -8.156 & 0.163 & 0.162 & { }^{4} \mathrm{H}_{5} \\ -2720.8436 & -2720.4688 & 3.76 & 0.002 & -6.752 & -8.209 & 0.178 & 0.163 & { }^{4} \mathrm{H}_{5}\end{array}$

\section{Equilibrium Structures of ${ }^{5} \mathrm{H}_{4}$ and ${ }^{4} \mathrm{H}_{5}$ Global Minima}

Table SCZ1. Cartesian coordinates for the global minimum ${ }^{5} \mathrm{H}_{4}$ conformer of $\mathbf{1}$ at the PBE0/DEF2-TZVP/D3BJ/CPCM $\left(\mathrm{CH}_{3} \mathrm{CN}\right) / \mathrm{RIJCOSX}$ level.

\begin{tabular}{|c|c|c|c|}
\hline C & -0.1 & 61 & \\
\hline C & 029185396957 & 565847471 & 1. \\
\hline C & 877580478490 & 230 & 9898 \\
\hline C & 2.27787469709252 & 346 & 593 \\
\hline $\mathrm{C}$ & 237 & 27 & 11 \\
\hline 0 & 21 & 269 & 475 \\
\hline 0 & 027902971 & 566 & 125 \\
\hline C & 7799772718 & 402 & -0.35095 \\
\hline $\mathrm{O}$ & -0.4 & 10407 & 60461 \\
\hline $\mathrm{O}$ & 58 & 45 & 928 \\
\hline C & 24 & & 397 \\
\hline 0 & 69155114 & 367 & 5077 \\
\hline C & 364 & 640 & $-3.67 \varepsilon$ \\
\hline C & 32 & 00 & 176 \\
\hline C & & & \\
\hline C & & & 653 \\
\hline $\mathrm{O}$ & 1.5 & 887 & 3472 \\
\hline C & -2.2 & 105 & 28 \\
\hline $\mathrm{O}$ & -2.4 & -0 . & 499 \\
\hline $\mathrm{H}$ & -1.2 & 222 & 44 \\
\hline $\mathrm{H}$ & -0 & 876 & 646 \\
\hline $\mathrm{H}$ & & 7849 & 294 \\
\hline $\mathrm{H}$ & 3.27 & & \\
\hline $\mathrm{H}$ & 2.1 & 360 & $-0 . s$ \\
\hline $\mathrm{H}$ & 1.3 & 70 & 68 \\
\hline $\mathrm{H}$ & -0 . & 43368 & 460 \\
\hline $\mathrm{H}$ & 2214 & 617766 & -3.55722 \\
\hline $\mathrm{H}$ & -0.2 & 593844 & -4.22357 \\
\hline $\mathrm{H}$ & & 941 & -4.2 \\
\hline$\Pi$ & & & \\
\hline $\mathrm{H}$ & 2850028634 & 035339035 & 2730487 \\
\hline $\mathrm{H}$ & 9664031028 & 466914101 & 2.17829737034573 \\
\hline 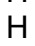 & & & \\
\hline & -2.14 & & 865 \\
\hline $\mathrm{H}$ & -3.30698482494287 & 3194374464 & 208560 \\
\hline
\end{tabular}

Table SCZ2. Cartesian coordinates for the global minimum ${ }^{4} \mathrm{H}_{5}$ conformer of $\mathbf{1}$ at the PBE0/DEF2-TZVP/D3BJ/CPCM $\left(\mathrm{CH}_{3} \mathrm{CN}\right) / \mathrm{RIJCOSX}$ level.

$\begin{array}{llll}\text { C } & -0.11084223014040 & 0.06877861018115 & 0.14659041362398 \\ \text { C } & -0.02221034327050 & -0.01720127044984 & 1.66532219604706 \\ \text { C } & 1.42759201974137 & 0.06701188251689 & 2.11359643251888 \\ \text { C } & 2.15535808859757 & 1.09086416596452 & 1.31566916727257\end{array}$




$\begin{array}{llcc}\text { C } & 1.63476957553070 & 1.63123167186977 & 0.21937681099351 \\ \text { O } & 0.44933883126245 & 1.28837147526798 & -0.32557365213899 \\ \text { O } & 1.48321110281129 & 0.47847973699098 & 3.49134978417840 \\ \text { C } & -1.53867643991696 & 0.01848742915704 & -0.32259181352093 \\ \text { O } & -0.57721806304409 & -1.27449261953405 & 2.04031392428026 \\ \text { O } & -1.52189517763831 & -0.09370117006356 & -1.74366994546384 \\ \text { C } & -2.71733047392876 & -0.13999170357456 & -2.34271912380676 \\ \text { O } & -3.75727848464594 & -0.09030275571849 & -1.73398047952446 \\ \text { C } & -2.57771712354397 & -0.26247627014049 & -3.82248505261619 \\ \text { C } & -1.35526416763202 & -1.33077550523185 & 3.13652439534165 \\ \text { C } & 1.60143212333516 & -0.46607407321065 & 4.43600869871175 \\ \text { C } & 1.60646540028455 & 0.14619789958291 & 5.79613886551610 \\ \text { O } & 1.68619272102964 & -1.64533192752885 & 4.20109064220885 \\ \text { C } & -1.80573093900880 & -2.72810182094709 & 3.38837410643634 \\ \text { O } & -1.63399364829279 & -0.36840199966416 & 3.80341263975096 \\ \text { H } & 0.45537609685468 & -0.76207426966783 & -0.29338486286870 \\ \text { H } & -0.59285319263026 & 0.79155984766951 & 2.12692813628792 \\ \text { H } & 1.88609425686519 & -0.92199236606562 & 2.03825466999445 \\ \text { H } & 3.12614421911763 & 1.42081311231825 & 1.66225691169027 \\ \text { H } & 2.12836903115910 & 2.41168417174952 & -0.34803140620533 \\ \text { H } & -2.06847396846051 & 0.92683449327038 & -0.02659197795558 \\ \text { H } & -2.04815397458336 & -0.84449155359399 & 0.10724843896643 \\ \text { H } & -2.04721505111233 & -1.18578225840242 & -4.06591758600890 \\ \text { H } & -3.55976526703603 & -0.26844968349736 & -4.28932853863698 \\ \text { H } & -1.98534471938076 & 0.56878860926011 & -4.21012450790073 \\ \text { H } & 0.61951656728486 & 0.56943817310490 & 5.99919082377203 \\ \text { H } & 1.83850512279139 & -0.60888743411309 & 6.54355309315242 \\ \text { H } & 2.32966569421673 & 0.96175783280481 & 5.84398827642182 \\ \text { H } & -2.33751351822544 & -3.11030890036591 & 2.51459459473728 \\ \text { H } & -0.93478433386154 & -3.36735325571731 & 3.54746354624168 \\ \text { H } & -2.45311973452954 & -2.75883827422161 & 4.26136137850280\end{array}$

Table SCZ3. Cartesian coordinates for the global minimum ${ }^{5} \mathrm{H}_{4}$ conformer of 2 at the PBE0/DEF2-TZVP/D3BJ/CPCM $\left(\mathrm{CH}_{3} \mathrm{CN}\right) / \mathrm{RIJCOSX}$ level.

\begin{tabular}{rrrr}
\hline$C$ & 1.638812 & -0.521008 & -0.132845 \\
$C$ & 0.459633 & -0.686164 & 0.804036 \\
$C$ & 0.620388 & 0.147322 & 2.079451 \\
$C$ & 2.035600 & 0.123791 & 2.533647 \\
$C$ & 3.017447 & -0.339852 & 1.762573 \\
O & 2.891813 & -0.783827 & 0.498005 \\
O & 0.211495 & 1.495100 & 1.880657 \\
$C$ & 1.698875 & 0.868587 & -0.749945 \\
O & 0.329167 & -2.060756 & 1.105585 \\
O & 0.464924 & 1.124183 & -1.367389 \\
$C$ & 0.252589 & 2.481982 & -1.645199 \\
$C$ & -0.988009 & -2.438380 & 1.461644 \\
$C$ & -1.184035 & 1.660294 & 1.853680 \\
$C$ & -1.536942 & 3.091758 & 1.584495 \\
$C$ & -1.988334 & -2.218536 & 0.358775 \\
$C$ & -1.177698 & 2.724296 & -2.023072 \\
$C$ & -2.137300 & 1.721248 & -1.964349 \\
$C$ & -3.460538 & 1.997121 & -2.285547 \\
$C$ & -3.839061 & 3.275490 & -2.665028 \\
$C$ & -2.882544 & 4.281974 & -2.724697 \\
$C$ & -1.563328 & 4.006000 & -2.407498 \\
$C$ & -1.737135 & -2.692043 & -0.927117 \\
$C$ & -2.652593 & -2.480711 & -1.945989 \\
$C$ & -3.836190 & -1.797222 & -1.690911 \\
C & -4.094000 & -1.321891 & -0.413997 \\
C & -3.169920 & -1.528039 & 0.602609 \\
$C$ & -0.608375 & 4.116377 & 1.720918 \\
& & &
\end{tabular}




$\begin{array}{rrrr}\text { C } & -0.972243 & 5.432249 & 1.462065 \\ \mathrm{C} & -2.266780 & 5.736558 & 1.068470 \\ \mathrm{C} & -3.198271 & 4.715088 & 0.929953 \\ \mathrm{C} & -2.833021 & 3.403474 & 1.182977 \\ \mathrm{H} & 1.551900 & -1.268807 & -0.923526 \\ \mathrm{H} & -0.416587 & -0.339347 & 0.251336 \\ \mathrm{H} & -0.014674 & -0.295693 & 2.859234 \\ \mathrm{H} & 2.277426 & 0.453545 & 3.536513 \\ \mathrm{H} & 4.043068 & -0.418804 & 2.106896 \\ \mathrm{H} & 2.519361 & 0.900689 & -1.478834 \\ \mathrm{H} & 1.900575 & 1.613171 & 0.028025 \\ \mathrm{H} & 0.917808 & 2.819629 & -2.453805 \\ \mathrm{H} & 0.494161 & 3.084599 & -0.758235 \\ \mathrm{H} & -1.320892 & -1.910072 & 2.363629 \\ \mathrm{H} & -0.923635 & -3.499980 & 1.713253 \\ \mathrm{H} & -1.615220 & 1.340375 & 2.815378 \\ \mathrm{H} & -1.645424 & 1.036043 & 1.077512 \\ \mathrm{H} & -1.850128 & 0.722063 & -1.661173 \\ \mathrm{H} & -4.199415 & 1.205517 & -2.232156 \\ \mathrm{H} & -4.873178 & 3.489355 & -2.911528 \\ \mathrm{H} & -3.167876 & 5.287151 & -3.015305 \\ \mathrm{H} & -0.821555 & 4.797946 & -2.447722 \\ \mathrm{H} & -0.812178 & -3.221666 & -1.129365 \\ \mathrm{H} & -2.445106 & -2.851493 & -2.943750 \\ \mathrm{H} & -4.551142 & -1.631995 & -2.489125 \\ \mathrm{H} & -5.009714 & -0.778751 & -0.208742 \\ \mathrm{H} & -3.367295 & -1.142336 & 1.598003 \\ \mathrm{H} & 0.405073 & 3.879770 & 2.020611 \\ \mathrm{H} & -0.236370 & 6.222354 & 1.566994 \\ \mathrm{H} & -2.547750 & 6.763135 & 0.860887 \\ \mathrm{H} & -4.208415 & 4.939628 & 0.606483 \\ \mathrm{H} & -3.558258 & 2.606907 & 1.050949 \\ & & & \end{array}$

Table SCZ4. Cartesian coordinates for the global minimum ${ }^{4} \mathrm{H}_{5}$ conformer of 2 at the PBE0/DEF2-TZVP/D3BJ/CPCM $\left(\mathrm{CH}_{3} \mathrm{CN}\right) / \mathrm{RIJCOSX}$ level.

\begin{tabular}{rrrr}
\hline$C$ & -0.348331 & 0.041473 & -0.383841 \\
$\mathrm{C}$ & 0.226819 & -0.185332 & 1.009440 \\
$\mathrm{C}$ & 1.693564 & -0.548040 & 0.901909 \\
$\mathrm{C}$ & 2.361374 & 0.354637 & -0.088359 \\
$\mathrm{C}$ & 1.663575 & 1.117267 & -0.926035 \\
$\mathrm{O}$ & 0.320077 & 1.116407 & -1.045527 \\
$\mathrm{O}$ & 2.270788 & -0.423666 & 2.193219 \\
$\mathrm{C}$ & -1.824241 & 0.330958 & -0.376921 \\
$\mathrm{O}$ & -0.493914 & -1.225392 & 1.623359 \\
$\mathrm{O}$ & -2.103124 & 1.420630 & 0.465876 \\
$\mathrm{C}$ & -3.488591 & 1.709856 & 0.553547 \\
$\mathrm{C}$ & -0.941082 & -0.930617 & 2.924454 \\
$\mathrm{C}$ & 3.394284 & -1.257227 & 2.392292 \\
$\mathrm{C}$ & 3.038730 & -2.717125 & 2.490357 \\
$\mathrm{C}$ & -1.753846 & -2.065791 & 3.463770 \\
$\mathrm{C}$ & -4.248554 & 0.699126 & 1.366342 \\
$\mathrm{C}$ & -4.196310 & 0.742578 & 2.757727 \\
$\mathrm{C}$ & -4.849936 & -0.211997 & 3.521171 \\
$\mathrm{C}$ & -5.570538 & -1.223947 & 2.900236 \\
$\mathrm{C}$ & -5.638304 & -1.271516 & 1.515862 \\
$\mathrm{C}$ & -4.980550 & -0.313850 & 0.754751 \\
$\mathrm{C}$ & -2.315077 & -3.025002 & 2.630970 \\
$\mathrm{C}$ & -3.100320 & -4.040704 & 3.158557 \\
$\mathrm{C}$ & -3.336323 & -4.107564 & 4.523803 \\
$\mathrm{C}$ & -2.776498 & -3.151287 & 5.361915 \\
$\mathrm{C}$ & -1.988631 & -2.140771 & 4.833610
\end{tabular}




$\begin{array}{rrrr}\mathrm{C} & 1.992862 & -3.130223 & 3.312065 \\ \mathrm{C} & 1.659248 & -4.471153 & 3.410446 \\ \mathrm{C} & 2.374661 & -5.422361 & 2.691726 \\ \mathrm{C} & 3.420442 & -5.020896 & 1.873095 \\ \mathrm{C} & 3.745405 & -3.673590 & 1.770351 \\ \mathrm{H} & -0.194925 & -0.868914 & -0.979886 \\ \mathrm{H} & 0.134145 & 0.743789 & 1.582079 \\ \mathrm{H} & 1.753552 & -1.598702 & 0.586863 \\ \mathrm{H} & 3.442338 & 0.425943 & -0.096879 \\ \mathrm{H} & 2.124392 & 1.823595 & -1.608152 \\ \mathrm{H} & -2.342899 & -0.573708 & -0.042153 \\ \mathrm{H} & -2.151069 & 0.547706 & -1.403766 \\ \mathrm{H} & -3.917785 & 1.783941 & -0.454392 \\ \mathrm{H} & -3.552114 & 2.693864 & 1.022597 \\ \mathrm{H} & -1.558145 & -0.021780 & 2.905308 \\ \mathrm{H} & -0.090186 & -0.730987 & 3.586918 \\ \mathrm{H} & 3.844974 & -0.917412 & 3.328874 \\ \mathrm{H} & 4.140680 & -1.111482 & 1.601549 \\ \mathrm{H} & -3.634876 & 1.533702 & 3.245079 \\ \mathrm{H} & -4.795922 & -0.169530 & 4.602978 \\ \mathrm{H} & -6.075775 & -1.975262 & 3.496290 \\ \mathrm{H} & -6.200206 & -2.058889 & 1.025511 \\ \mathrm{H} & -5.037493 & -0.351389 & -0.328808 \\ \mathrm{H} & -2.135985 & -2.972052 & 1.564631 \\ \mathrm{H} & -3.534141 & -4.782593 & 2.496520 \\ \mathrm{H} & -3.950950 & -4.900958 & 4.934745 \\ \mathrm{H} & -2.950205 & -3.196005 & 6.431700 \\ \mathrm{H} & -1.549862 & -1.397576 & 5.492830 \\ \mathrm{H} & 1.430507 & -2.390148 & 3.868806 \\ \mathrm{H} & 0.836374 & -4.776451 & 4.047698 \\ \mathrm{H} & 2.113323 & -6.471994 & 2.767454 \\ \mathrm{H} & 3.979302 & -5.755809 & 1.304226 \\ \mathrm{H} & 4.554107 & -3.360415 & 1.117315 \\ & & & \end{array}$

Table SCZ5. Cartesian coordinates for the global minimum ${ }^{5} \mathrm{H}_{4}$ conformer of $\mathbf{3}$ at the PBE0/DEF2-TZVP/D3BJ/CPCM $\left(\mathrm{CH}_{3} \mathrm{CN}\right) / \mathrm{RIJCOSX}$ level.

$\begin{array}{lllc}\mathrm{C} & 0.00298350588312 & 0.05849325267098 & 0.01163579437021 \\ \mathrm{C} & 0.04798349857172 & -0.00673988673588 & 1.53373979841776 \\ \mathrm{C} & 1.48977245179040 & -0.01757807136129 & 2.04322802863407 \\ \mathrm{C} & 2.32720529079795 & -0.88888982551919 & 1.17639265185697 \\ \mathrm{C} & 1.89041250107082 & -1.33892293036597 & 0.00090682470430 \\ \mathrm{O} & 0.71742456748106 & -1.03110794454091 & -0.57845447292182 \\ \mathrm{O} & 2.04460632246486 & 1.28805022976570 & 2.08535092417645 \\ \mathrm{C} & 0.49856457046124 & 1.37508173636202 & -0.54217010283947 \\ \mathrm{O} & -0.56205476056818 & -1.17843247349350 & 2.02264432540993 \\ \mathrm{O} & 0.09472316939193 & 1.47200452284507 & -1.88504397948724 \\ \mathrm{C} & 0.57619877014771 & 2.64335685778806 & -2.49045144259666 \\ \mathrm{C} & -1.96734885327814 & -1.16754380380456 & 1.95512778845021 \\ \mathrm{C} & 1.65440871140057 & 2.02368785523324 & 3.22055225787606 \\ \mathrm{H} & -1.02746917062419 & -0.07109533851754 & -0.32540355120610 \\ \mathrm{H} & -0.47419279419139 & 0.87601249410727 & 1.92453336414853 \\ \mathrm{H} & 1.46606855864317 & -0.42689122422759 & 3.06279876800969 \\ \mathrm{H} & 3.30027120962112 & -1.20926979952310 & 1.52760427971248 \\ \mathrm{H} & 2.46404661453685 & -2.02932790799292 & -0.60870601105642 \\ \mathrm{H} & 0.07295336088414 & 2.20017036413017 & 0.04694364253171 \\ \mathrm{H} & 1.59043282178744 & 1.44205155465038 & -0.46053907055803 \\ \mathrm{H} & 1.67430731710701 & 2.67386493721446 & -2.49302132364527 \\ \mathrm{H} & 0.22152742308093 & 2.65487635779011 & -3.52205500856468 \\ \mathrm{H} & 0.20744379481888 & 3.54361359485377 & -1.97967817799828 \\ \mathrm{H} & -2.38270635081845 & -0.27869697461279 & 2.44748704794406\end{array}$


H $\quad-2.33976462917594$

H $\quad-2.32154546455083$

H 2.13344465574768

H 0.56925623292119

H 1.98052867459735
$-1.20243177965091$

$-2.05728514353459$

3.00129390640747

2.17451389103751

1.53005854902450
0.92477171135190

2.47705001300946

3.15395499816694

3.27315143852087

4.14515348358233

Table SCZ6. Cartesian coordinates for the global minimum ${ }^{4} \mathrm{H}_{5}$ conformer of 3 at the PBE0/DEF2-TZVP/D3BJ/CPCM $\left(\mathrm{CH}_{3} \mathrm{CN}\right) / \mathrm{RIJCOSX}$ level.

\begin{tabular}{cccc}
\hline C & -0.03552877546462 & 0.11738826455875 & 0.09261375624355 \\
C & 0.10137524238219 & -0.03025186314947 & 1.60364081642334 \\
C & 2.04475301973410 & 1.45046263272288 & 1.28602290395663 \\
C & 1.40301129710797 & 1.96268558566017 & 0.23898250418485 \\
O & 0.29596511921764 & 1.44237440084005 & -0.32716184608537 \\
O & 1.52971710509814 & 0.45526074729621 & 3.42681787477841 \\
C & -1.41637417426968 & -0.19803280815561 & -0.41430438722831 \\
O & -0.26552311571692 & -1.34157334170147 & 1.96509798830452 \\
O & -2.36925286693971 & 0.58414826251663 & 0.25626460349471 \\
C & -3.67512126036312 & 0.30450370545367 & -0.17854782449299 \\
C & -1.27277831525104 & -1.41038342830396 & 2.94787059027380 \\
C & 2.79168004650176 & 0.25644279186989 & 4.01404494920521 \\
H & 0.66598250548778 & -0.57420740492638 & -0.39507842767796 \\
H & -0.55451047861783 & 0.70176808378426 & 2.08779616040584 \\
H & 2.13277061853135 & -0.64067105845830 & 1.80182903985257 \\
H & 2.94231896245743 & 1.94591404401644 & 1.63621239386310 \\
H & 1.72318688991039 & 2.86686232548629 & -0.26819010550444 \\
H & -1.45109891186847 & -0.00934987057881 & -1.49706219288320 \\
H & -1.61056386476122 & -1.26661968313357 & -0.25328294389298 \\
H & -3.79505747054050 & 0.51649172595229 & -1.24982372731406 \\
H & -3.94101384039706 & -0.74665150276184 & -0.00042121063018 \\
H & -4.35602294225940 & 0.94249799684190 & 0.38682847389896 \\
H & -0.93469397515390 & -0.98018735682354 & 3.89761540054299 \\
H & -2.18431038452638 & -0.89282372055050 & 2.62582118094159 \\
H & -1.50311295639941 & -2.46631896781192 & 3.10079182962234 \\
H & 3.15053160674313 & -0.76793173011543 & 3.84616532253977 \\
H & 3.54437401097565 & 0.95713841706967 & 3.63108300790093 \\
H & 2.68068378682289 & 0.42088198961003 & 5.08703995296542
\end{tabular}

Table SCZ7. Cartesian coordinates for the global minimum ${ }^{5} \mathrm{H}_{4}$ conformer of 4 at the PBE0/DEF2-TZVP/D3BJ/CPCM $\left(\mathrm{CH}_{3} \mathrm{CN}\right) / \mathrm{RIJCOSX}$ level.
C -0.07572133894560
$-0.01344536860228$
0.02654957764489
C 0.01177730617736
$-0.01623678472247$
1.54047058512022
C 1.46473425311553
0.03844102578996
2.02867949555732
C 2.32002056165913
$-0.81982906217886$
1.16500738397306
C 1.89006471837703
$-1.30487194699212$
O 0.69448216308639
$-1.06011760949993$
0.00191862158696
O 1.98629705547943
1.35790467728344
1.30177406758400
$-0.56672063286877$
2.04594948196374
C 0.36078860466080
$-1.18994949215224$
$-0.59838153225087$
O -0.61649876382846
2.00876051056723
$2.33728919871013 \quad-0.05418792190111$
O -0.41136630953109
3.61209747837366
$-0.45901680822112$
C -1.20097663364697
$-1.04063004201459$
3.28801150743390
2.13113743861714
3.14364160335476
C 1.54664500056142
4.66365535662501
$-2.36859530898502$
3.48835249415961
0.22602712584107
3.72402226453909
3.06595962310109
C 2.19325397622717
H -1.11000913582446
$-0.22399966606615$
$-0.25365488468645$
0.86858283976458
1.88912341330798
H -0.52982129810629
$-0.35716093208276$
3.05478081399966 
H 3.31089332446466

H 2.48364837714428

H 0.21671970049406

H 1.42641651088425

H $\quad-0.04499600277732$

H 1.09918905332251

H -1.99555201340585

H $\quad-0.45523656163848$

H 1.82006649169649

H 0.45367511017954

H -0.45581126246533

H -1.84802010586891

H -0.70833636952670

H $\quad-2.23441324851334$

H -0.96581176527730

H $\quad-2.50685431742902$

H 1.91282008004945

H 1.87325611216516

H 3.28226128126960
$-1.09507295013503$

$-1.97952188159282$

1.23620548184921

1.47283468912898

3.70596454848778

3.72643165833617

$-0.28255182195343$

$-0.68601674137083$

1.62584404084914

2.23467076847686

5.65934425103692

4.56569659346780

4.58222641075079

$-2.27068265328031$

$-3.11674377919660$

$-2.72799446258752$

4.00051722069295

4.10455608713812

3.40345817629072
1.50535988274359

$-0.60596287944628$

$-1.68600752829451$

$-0.40503995675531$

$-1.55199518865517$

$-0.20203355380651$

3.24017036922740

4.01275334496024

4.08152872556851

3.13647717228148

$-0.07042358387191$

$-0.04518997473318$

1.31244878179102

4.70260020405694

3.80141722069317

3.01341920090253

2.14261747304213

3.90957306053929

3.09585500169389

Table SCZ8. Cartesian coordinates for the global minimum ${ }^{4} \mathrm{H}_{5}$ conformer of 4 at the PBE0/DEF2-TZVP/D3BJ/CPCM $\left(\mathrm{CH}_{3} \mathrm{CN}\right) / \mathrm{RIJCOSX}$ level.

$\begin{array}{llcc}\text { C } & 0.05192096443614 & 0.10209867655327 & -0.02941792665681 \\ \text { C } & -0.03865867717161 & 0.02853794907943 & 1.49175301847928 \\ \text { C } & 1.29902284511980 & 0.38028678220876 & 2.11060704059297 \\ \text { C } & 1.40818541125307 & 1.99801119433178 & 0.24015584922808 \\ \text { O } & 0.42460047649824 & 1.40954375511376 & -0.46887378065311 \\ \text { O } & 1.07677759849576 & 0.64116998343323 & 3.48346611999841 \\ \text { C } & -1.23204784043762 & -0.26606478147951 & -0.72269398755590 \\ \text { O } & -0.41055479802117 & -1.27648677985729 & 1.86426882215014 \\ \text { O } & -2.28295478895897 & 0.53640144833504 & -0.25573595847762 \\ \text { C } & -3.51537647805208 & 0.20720706399153 & -0.85795380755359 \\ \text { C } & -1.63722721833528 & -1.36356605287300 & 2.56732298010867 \\ \mathrm{C} & 2.23460325079039 & 0.50281922039613 & 4.27995085631581 \\ \mathrm{C} & -4.58553183399475 & 1.11679316783336 & -0.31688267340122 \\ \mathrm{C} & -1.92786411555728 & -2.81418365219007 & 2.84193041680954 \\ \mathrm{C} & 1.87089957338382 & 0.78067016683674 & 5.71419172856759 \\ \mathrm{H} & 0.82562231125151 & -0.60166437131330 & -0.36653361293450 \\ \mathrm{H} & -0.77903278732828 & 0.75805509590871 & 1.83714924145142 \\ \mathrm{H} & 1.95521203628431 & -0.49911055945284 & 2.02291971062115 \\ \mathrm{H} & 2.69740959046969 & 2.11124779805363 & 1.86587344039088 \\ \mathrm{H} & 1.77903761966131 & 2.88866448733681 & -0.25591229752166 \\ \mathrm{H} & -1.43646471291166 & -1.32853638769901 & -0.53583949354235 \\ \mathrm{H} & -1.09854540876057 & -0.13839802598694 & -1.80641932124146 \\ \mathrm{H} & -3.76066056141748 & -0.84547403199249 & -0.65232917711345 \\ \mathrm{H} & -3.43452468401018 & 0.31367690494258 & -1.94979161974020 \\ \mathrm{H} & -2.44140040920718 & -0.91432073133679 & 1.97129987302578 \\ \mathrm{H} & -1.56339459402634 & -0.79998162367022 & 3.50630187688096 \\ \mathrm{H} & 3.01818135366573 & 1.19698093149470 & 3.94692423331111 \\ \mathrm{H} & 2.63409294961526 & -0.51610127810137 & 4.17247988753941 \\ \mathrm{H} & -5.54672359050337 & 0.87742624212367 & -0.77793126802682 \\ \mathrm{H} & -4.68720997821254 & 1.00184476625880 & 0.76504596802368 \\ \mathrm{H} & -4.35295197973699 & 2.16221093436171 & -0.53378848463094 \\ \mathrm{H} & -2.86639529165714 & -2.90830571347108 & 3.39341330496542 \\ \mathrm{H} & -1.13215944477854 & -3.26466593417289 & 3.44051111671595 \\ \mathrm{H} & -2.02051720137979 & -3.37670298372124 & 1.90946278131827 \\ \mathrm{H} & 1.10266013756371 & 0.08571423247345 & 6.06232133231462 \\ \mathrm{H} & 2.75105261875429 & 0.66660862337701 & 6.35156516024159 \\ & 1.49450419795611 & 1.79997314159417 & 5.83037880849023\end{array}$


Table SCZ9. Cartesian coordinates for the global minimum ${ }^{5} \mathrm{H}_{4}$ conformer of 5 at the PBE0/DEF2-TZVP/D3BJ/CPCM $\left(\mathrm{CH}_{3} \mathrm{CN}\right) / \mathrm{RIJCOSX}$ level.

\begin{tabular}{|c|c|c|c|}
\hline & 0.78946391634184 & -1.14664456713461 & -0.39947768839495 \\
\hline & 0.53264862390943 & -0.59514015193578 & 364404 \\
\hline C & 1.82512124787202 & -0.15882311811276 & 1.68500866533238 \\
\hline 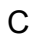 & 2.89860143690950 & -1.15909584170339 & 1.43069656062148 \\
\hline C & 2.79796615818233 & -2.05681504148554 & 0.45333293129066 \\
\hline & 1.77421468461017 & -2.17922874175307 & -0.41232147045690 \\
\hline $\mathrm{O}$ & 2.22326246398198 & 1.12355782428234 & 1.22811434003212 \\
\hline $0_{2}$ & 1.16414154663879 & -0.08105866702290 & -1.41761378682078 \\
\hline a $>$ & -0.09424573664088 & -1.58540987275134 & 1.7791090 \\
\hline $\mathrm{O}$ & 0.11760403364772 & 0.85460841415188 & -1.47275039634951 \\
\hline & -0.39465775980875 & 1.58385064059179 & -2.87588898583941 \\
\hline $\mathrm{Si}$ & -1.71926147737754 & -1.51343047714822 & 2.14738008592081 \\
\hline $\mathrm{Si}$ & 2.20563396256426 & 2.49816380850263 & 2.16433562577915 \\
\hline C & 0.44181850302085 & 3.03265839267624 & 2.50792987890777 \\
\hline C & 3.10052273061865 & 2.15369140252893 & 3.77835510853184 \\
\hline C & 3.09830099700105 & 3.78532660366919 & 1.14428 \\
\hline C & 3.28295070141754 & 3.38208321227595 & 4.66610254151604 \\
\hline C & 4.51409629769259 & 3.39835460956286 & 0.73130074736863 \\
\hline & 06793739428 & & \\
\hline C & -2.75188742534777 & -1.45411075097732 & 0.58052514405214 \\
\hline C & -2.03534818458881 & -3.06727475560516 & 3.13450280485328 \\
\hline C & -3.47173840541731 & 940160838719 & \\
\hline C & -2.79901198429519 & -2.75083060022714 & -0.22093644319823 \\
\hline C & -2.07787168515419 & 0.03006340037958 & 3.14955032174538 \\
\hline C & -1.32924360172072 & 0.11967117085363 & 4.47496517144839 \\
\hline C & 1.02421325577432 & 2.54016574688871 & -3.64053393370785 \\
\hline C & -1.00230411534923 & 0.28506502735631 & -4.08450467123609 \\
\hline C & -1.78145047059711 & 2.70574632163604 & -2.31508715566771 \\
\hline C & -2.04342582555748 & -0.66947572799170 & -3.50809556469398 \\
\hline C & 1.68467984438353 & 3.53118244699117 & -2.68758772103396 \\
\hline C & -2.47505740047539 & 3.45879609654282 & -3.44597976932446 \\
\hline & 7269 & -1.62838246147472 & -0.74026175274199 \\
\hline $\mathrm{H}$ & -0.10002747435275 & 0.29014968998864 & 0.87359213998620 \\
\hline $\mathrm{H}$ & 1.60668055659716 & -0.13016852534199 & 30008978 \\
\hline $\mathrm{H}$ & 3.78379946921197 & 19736 & 7863 \\
\hline $\mathrm{H}$ & 3.55556270285068 & -2.81149769160921 & 0.26984914810309 \\
\hline $\mathrm{H}$ & 1.321 & & 83043 \\
\hline $\mathrm{H}$ & 2.09900015191460 & 0.40924568435303 & -1.13129909822458 \\
\hline $\mathrm{H}$ & -0.04186893822338 & 2.23796712825580 & 476769732 \\
\hline $\mathrm{H}$ & 820253195 & 3.90275332620983 & 5971441391 \\
\hline $\mathrm{H}$ & 4.07547552346974 & 1.71274529406643 & 3.53876409920868 \\
\hline & 2.55 & & \\
\hline $\mathrm{H}$ & 2.49067712269592 & 3.99 & 0.25700341511132 \\
\hline $\mathrm{H}$ & 3.1088731 & 10841 & 93098693 \\
\hline $\mathrm{H}$ & 3.8071215 & 3.13 & o \\
\hline $\mathrm{H}$ & 3.86417923667930 & 4.16037002840996 & 21892585 \\
\hline $\mathrm{H}$ & 2.32242712934344 & & \\
\hline & 4.98424623000240 & 4.17407419942272 & 3848736137 \\
\hline $\mathrm{H}$ & 5.1579 & 46281 & 1.6 \\
\hline $\mathrm{H}$ & 4.51696902952170 & 2.47299473460317 & 3830898546 \\
\hline $\mathrm{H}$ & -1.42125143233169 & 3.55291723008344 & 1.49198008150130 \\
\hline $\mathrm{H}$ & 1392961 & 4.23173354941405 & 0.7432474173065 \\
\hline & 9349 & 2.52743180598327 & 0.53667007826724 \\
\hline $\mathrm{H}$ & -2.37602900226762 & -0.63168621292867 & \\
\hline $\mathrm{H}$ & -3.76774269551017 & -1.15463244149433 & 0.865550309567 \\
\hline & -1.76792578747192 & -3.92783214056889 & 2.51043121107411 \\
\hline $\mathrm{H}$ & -1.33754354612732 & -3.08311236035504 & \\
\hline $\mathrm{H}$ & -3.62117321680480 & -4.12839826796700 & 4.190315642299 \\
\hline & -3.74506422614990 & -2.37521854890728 & 4.297772451658 \\
\hline
\end{tabular}




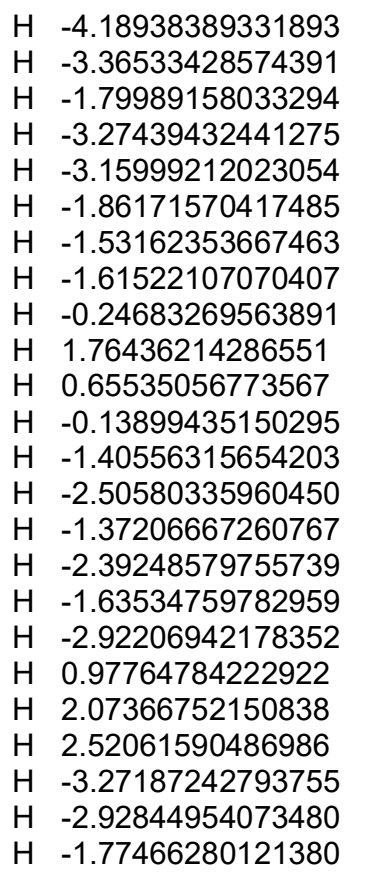

$-3.19851644691559$ $-2.63365592844486$ $-3.10466305321235$ $-3.55335535949362$ 0.06790927012612 0.90502673720320 1.05997622636261 $-0.69218046397563$ 0.04883639167529 1.81560839346649 3.05386951879957 $-0.27923981747140$ 0.80828521894017 2.09707091761089 3.41295881257734 $-1.39009503450740$ $-1.24080206341996$ $-0.13627383181297$ 4.28928723299620 3.02290813891429 4.05665809564661 4.10673509974290 2.77527242707707 4.09429934505346
2.80707363644285 $-1.14937058545309$ $-0.49135571458165$ 0.34952076711591 3.32399936786978 2.52412633905332 4.99604687382161 5.14953212979646 4.33561025753853

$-4.00233459826957$ $-4.53671368349893$

$-4.45711343619895$

$-4.96042957037900$

$-1.76123994433997$

$-1.58556843727403$

$-4.25361587578523$

$-2.67084066734407$

$-3.13381381867532$

$-2.33892396759943$

$-1.80170741382368$

$-3.15893868973649$

$-3.06933605380665$

$-4.16948062579241$

$-3.99596176083212$

Table SCZ10. Cartesian coordinates for the global minimum ${ }^{5} \mathrm{H}_{4}$ conformer of 6 at the PBE0/DEF2-TZVP/D3BJ/CPCM $\left(\mathrm{CH}_{3} \mathrm{CN}\right) / \mathrm{RIJCOSX}$ level.

\begin{tabular}{rrrr}
\hline C & 0.617660 & -0.776524 & -0.439913 \\
C & -0.265286 & -0.473798 & 0.755495 \\
C & 0.530734 & 0.132443 & 1.918447 \\
C & 1.887162 & -0.467659 & 2.003415 \\
C & 2.368407 & -1.264015 & 1.051231 \\
O & 1.754472 & -1.572148 & -0.106090 \\
O & 0.630113 & 1.544086 & 1.768782 \\
C & 1.100029 & 0.477703 & -1.149952 \\
O & -0.906700 & -1.679139 & 1.118217 \\
O & -0.028229 & 1.165411 & -1.618343 \\
C & 0.267578 & 2.468444 & -2.047538 \\
C & -2.089477 & -1.514926 & 1.878914 \\
C & -0.533353 & 2.217915 & 2.206173 \\
C & -0.993636 & 3.208485 & -2.370785 \\
C & -3.149765 & -0.697177 & 1.193911 \\
C & -0.665320 & 3.546700 & 1.529530 \\
C & 0.446877 & 4.317639 & 1.195734 \\
C & 0.300454 & 5.538064 & 0.564275 \\
C & -0.971036 & 6.026029 & 0.260261 \\
C & -2.089568 & 5.274925 & 0.601034 \\
C & -1.922097 & 4.042199 & 1.217502 \\
C & -3.472174 & -0.906017 & -0.147971 \\
C & -4.437989 & -0.144776 & -0.774537 \\
C & -5.119578 & 0.847281 & -0.065972 \\
C & -4.819395 & 1.060997 & 1.273985 \\
C & -3.833533 & 0.292107 & 1.883236 \\
C & -2.240162 & 2.738636 & -1.992904 \\
C & -3.396684 & 3.466755 & -2.250729 \\
C & -3.307253 & 4.695884 & -2.891551 \\
C & -2.057726 & 5.174958 & -3.286389 \\
C & -0.921252 & 4.436229 & -3.027383 \\
O & -4.367605 & 5.493387 & -3.167256 \\
C & -5.633804 & 5.075931 & -2.703107 \\
O & -6.040419 & 1.552658 & -0.760531
\end{tabular}




\begin{tabular}{rrrr} 
C & -6.764431 & 2.548005 & -0.065293 \\
$\mathrm{O}$ & -1.020410 & 7.222374 & -0.367338 \\
$\mathrm{C}$ & -2.294871 & 7.756373 & -0.658899 \\
$\mathrm{H}$ & 0.040170 & -1.381454 & -1.141943 \\
$\mathrm{H}$ & -1.001894 & 0.260878 & 0.416381 \\
$\mathrm{H}$ & -0.005336 & -0.083064 & 2.852583 \\
$\mathrm{H}$ & 2.486509 & -0.298387 & 2.889601 \\
$\mathrm{H}$ & 3.325330 & -1.767873 & 1.136733 \\
$\mathrm{H}$ & 1.753527 & 0.181413 & -1.981756 \\
$\mathrm{H}$ & 1.679298 & 1.107224 & -0.465092 \\
$\mathrm{H}$ & 0.810094 & 3.002548 & -1.253903 \\
$\mathrm{H}$ & 0.927775 & 2.441392 & -2.927507 \\
$\mathrm{H}$ & -1.873254 & -1.071333 & 2.859020 \\
$\mathrm{H}$ & -2.444832 & -2.533004 & 2.060683 \\
$\mathrm{H}$ & -0.489797 & 2.334936 & 3.299959 \\
$\mathrm{H}$ & -1.430443 & 1.627323 & 1.988148 \\
$\mathrm{H}$ & 1.441348 & 3.946748 & 1.416164 \\
$\mathrm{H}$ & 1.166860 & 6.129343 & 0.289010 \\
$\mathrm{H}$ & -3.086560 & 5.617737 & 0.360688 \\
$\mathrm{H}$ & -2.801278 & 3.445580 & 1.439762 \\
$\mathrm{H}$ & -2.945068 & -1.667635 & -0.712799 \\
$\mathrm{H}$ & -4.678381 & -0.299653 & -1.820362 \\
$\mathrm{H}$ & -5.328054 & 1.824464 & 1.847178 \\
$\mathrm{H}$ & -3.594124 & 0.479648 & 2.925445 \\
$\mathrm{H}$ & -2.317821 & 1.792431 & -1.472836 \\
$\mathrm{H}$ & -4.351217 & 3.067090 & -1.935615 \\
$\mathrm{H}$ & -1.996298 & 6.134066 & -3.788170 \\
$\mathrm{H}$ & 0.044860 & 4.826998 & -3.332442 \\
$\mathrm{H}$ & -6.341065 & 5.846710 & -3.005937 \\
$\mathrm{H}$ & -5.933125 & 4.122520 & -3.149883 \\
$\mathrm{H}$ & -5.646310 & 4.990307 & -1.611972 \\
$\mathrm{H}$ & -7.445867 & 2.992348 & -0.788732 \\
$\mathrm{H}$ & -7.347009 & 2.116304 & 0.754446 \\
$\mathrm{H}$ & -6.103655 & 3.323114 & 0.335220 \\
$\mathrm{H}$ & -2.865508 & 7.098932 & -1.320955 \\
$\mathrm{H}$ & -2.870066 & 7.938163 & 0.254940 \\
$\mathrm{H}$ & -2.120052 & 8.705026 & -1.164480 \\
& & & \\
\hline & & &
\end{tabular}

Table SCZ11. Cartesian coordinates for the global minimum ${ }^{4} \mathrm{H}_{5}$ conformer of 6 at the PBE0/DEF2-TZVP/D3BJ/CPCM $\left(\mathrm{CH}_{3} \mathrm{CN}\right) / \mathrm{RIJCOSX}$ level.

\begin{tabular}{rrrr}
\hline $\mathrm{C}$ & -0.485848 & -0.542139 & 1.238000 \\
$\mathrm{C}$ & 0.662126 & 0.108617 & 2.003715 \\
$\mathrm{C}$ & 2.020487 & -0.436071 & 1.547158 \\
$\mathrm{C}$ & 2.015006 & -0.791582 & 0.098579 \\
$\mathrm{C}$ & 0.908682 & -0.771034 & -0.637870 \\
$\mathrm{O}$ & -0.321692 & -0.484497 & -0.178555 \\
$\mathrm{O}$ & 3.110314 & 0.410805 & 1.915349 \\
$\mathrm{C}$ & -1.820359 & 0.082985 & 1.548158 \\
$\mathrm{O}$ & 0.440768 & -0.220366 & 3.361382 \\
$\mathrm{O}$ & -1.807223 & 1.426353 & 1.147808 \\
$\mathrm{C}$ & -2.990942 & 2.099727 & 1.488359 \\
$\mathrm{C}$ & 0.836386 & 0.767645 & 4.304607 \\
$\mathrm{C}$ & 3.516692 & 1.435036 & 1.015220 \\
$\mathrm{C}$ & -3.054542 & 3.420486 & 0.785380 \\
$\mathrm{C}$ & -0.158429 & 1.879807 & 4.452166 \\
$\mathrm{C}$ & 2.811866 & 2.752702 & 1.170678 \\
$\mathrm{C}$ & 1.667534 & 3.054094 & 0.442818 \\
$\mathrm{C}$ & 1.006906 & 4.265285 & 0.589014 \\
$\mathrm{C}$ & 1.502685 & 5.214457 & 1.477455 \\
$\mathrm{C}$ & 2.668472 & 4.943425 & 2.193736 \\
$\mathrm{C}$ & 3.304352 & 3.725175 & 2.041533
\end{tabular}




$\begin{array}{lrrr} & & & \\ \mathrm{C} & -1.384109 & 1.668997 & 5.086931 \\ \mathrm{C} & -2.303220 & 2.690336 & 5.220294 \\ \mathrm{C} & -2.015888 & 3.961721 & 4.716533 \\ \mathrm{C} & -0.107876 & 4.188520 & 4.082260 \\ \mathrm{C} & -2.474052 & 3.144831 & 3.954901 \\ \mathrm{C} & -2.568392 & 4.818224 & -0.460836 \\ \mathrm{C} & -3.262158 & 5.875811 & -1.133370 \\ \mathrm{C} & -3.854268 & 5.700592 & 0.697784 \\ \mathrm{C} & -3.742615 & 4.489552 & 1.355087 \\ \mathrm{O} & -3.394284 & 7.099311 & -1.112411 \\ \mathrm{C} & -2.797958 & 7.311537 & -2.375667 \\ \mathrm{O} & -2.971340 & 4.901976 & 4.887704 \\ \mathrm{C} & -2.689629 & 6.211818 & 4.438056 \\ \mathrm{O} & 0.912866 & 6.407154 & 1.714433 \\ \mathrm{C} & -0.300717 & 6.674023 & 1.037991 \\ \mathrm{H} & -0.528625 & -1.600629 & 1.529539 \\ \mathrm{H} & 0.616921 & 1.190048 & 1.854743 \\ \mathrm{H} & 2.197772 & -1.338496 & 2.144054 \\ \mathrm{H} & 2.946577 & -1.079249 & -0.374208 \\ \mathrm{H} & 0.900935 & -0.993671 & -1.699853 \\ \mathrm{H} & -2.008132 & -0.010965 & 2.624394 \\ \mathrm{H} & -2.609280 & -0.467696 & 1.015893 \\ \mathrm{H} & -3.860004 & 1.486113 & 1.199164 \\ \mathrm{H} & -3.051200 & 2.246909 & 2.574580 \\ \mathrm{H} & 1.817748 & 1.171355 & 4.038498 \\ \mathrm{H} & 0.940386 & 0.228250 & 5.249521 \\ \mathrm{H} & 3.418081 & 1.090958 & -0.018163 \\ \mathrm{H} & 4.581727 & 1.574540 & 1.216762 \\ \mathrm{H} & 1.267136 & 2.324031 & -0.253404 \\ \mathrm{H} & 0.103080 & 4.447949 & 0.024972 \\ \mathrm{H} & 3.051903 & 5.692015 & 2.877902 \\ \mathrm{H} & 4.206133 & 3.523942 & 2.611637 \\ \mathrm{H} & -1.620036 & 0.687681 & 5.486854 \\ \mathrm{H} & -3.253694 & 2.525913 & 5.715614 \\ \mathrm{H} & -0.54991 & 5.162199 & 3.684184 \\ \mathrm{H} & 1.050563 & 3.330782 & 3.453787 \\ \mathrm{H} & -1.921732 & 2.789236 & -0.912142 \\ \mathrm{H} & -2.090386 & 4.928103 & -2.097930 \\ \mathrm{H} & -4.383418 & 6.532481 & 1.148987 \\ \mathrm{H} & -4.193226 & 4.375225 & 2.335980 \\ \mathrm{H} & -3.015133 & 8.344009 & -2.645167 \\ \mathrm{H} & -1.712754 & 7.173113 & -2.336560 \\ \mathrm{H} & -3.220742 & 6.646441 & -3.135318 \\ \mathrm{H} & -3.572763 & 6.807905 & 4.662448 \\ \mathrm{H} & -1.826358 & 6.635225 & 4.961098 \\ \mathrm{H} & -2.508955 & 6.236966 & 3.359483 \\ \mathrm{H} & -0.655160 & 7.633785 & 1.410763 \\ \mathrm{H} & -0.142682 & 6.744676 & -0.042696 \\ \mathrm{H} & -1.048259 & 5.903074 & 1.241686 \\ & & & \\ & & & \end{array}$

Table SCZ12. Cartesian coordinates for the global minimum ${ }^{5} \mathrm{H}_{4}$ conformer of 7 at the PBE0/DEF2-TZVP/D3BJ/CPCM $\left(\mathrm{CH}_{3} \mathrm{CN}\right) / \mathrm{RIJCOSX}$ level.

\begin{tabular}{cccc}
\hline $\mathrm{C}$ & -0.38088883647486 & -0.44539368776705 & 0.30884120211366 \\
$\mathrm{C}$ & 0.65047604097015 & -0.27451792318815 & 1.41202048450178 \\
$\mathrm{C}$ & 2.04231563405579 & 0.02268847579569 & 0.87100728310450 \\
$\mathrm{C}$ & 2.30290507498905 & -0.72317361589921 & -0.37992525250060 \\
$\mathrm{C}$ & 1.33112531247375 & -1.37617048661957 & -1.01355156684375 \\
$\mathrm{O}$ & 0.02965593082421 & -1.39252405884210 & -0.66736087819111 \\
$\mathrm{O}$ & 2.14345003173395 & 1.44303036665455 & 0.60246806823152 \\
$\mathrm{C}$ & -0.72108690310712 & 0.84673859016844 & -0.41391244989878
\end{tabular}


O $\quad 0.77026675253139$

O -1.27673834680688

C -1.66485956356128

O -1.53340516988370

O -2.22458434969950

C -0.09331365023443

C 2.46646964290315

O 2.48916997943292

O 2.70976371596984

O 0.15402991266609

O -0.92500813018560

C $\quad-2.74951578066084$

C -4.26298535835219

Cl -4.99515733928827

Cl -4.83968353447906

Cl -4.70819993989885

C -0.72979805349311

C -1.61408799874439

Cl -2.68525627956547

Cl -0.63450403125745

Cl -2.57686125872195

C 2.75429106941051

C 1.47954020596795

Cl 0.78760318398804

Cl 0.28084216073248

Cl 1.91581043071891

H -1.29598842086026

H 0.32404338953149

H 2.77548204523135

H 3.31570117681033

H 1.51337970563013

H 0.15334765573216

H -1.46447603111720

H -2.33483725948333

H -2.50505395263079

H -1.36123429605058

H -0.13113659958252

H 3.47117825805900

H 3.15899277377699
$-1.50488878209512$ 1.72812000412847 2.90737206781306 3.31312723723869 3.56053947486216 $-1.67819704132112$ 2.20788523365962 3.46894368325778 1.84202032340342

$-2.87925590966326$

$-0.89530430518690$ 4.83984152553472

4.82297457138631

3.69105846387025

6.46328991896520

4.35232175008414

$-3.32482561227079$

$-4.44188979905482$

$-4.93671543849431$

$-5.82143285562159$

$-3.86950559615229$

4.48080720959615

5.24914057545770

5.94238320604298

4.18919885441856

6.54745472747345

$-0.84967726640239$

0.51235168036284

$-0.22472123315012$

$-0.79517117880823$

$-1.99606634525908$

1.30269895338485

0.63588680612462

5.53647204213733

5.16126367044875

$-2.51072547618363$

$-3.71942133445736$

5.16992950295900

4.06281203120828
2.13423525435364

0.56372185074317

0.10639518696703

$-1.01400911940675$

1.12953922733622

3.12660326819488

1.63567047569989

1.19196306359744

2.75173463158974

3.65467864047887

3.49010964360210 0.86305377107357

1.02535095624944

$-0.12151714536202$

0.70155097281795

2.67230807224947

4.66132205281612

4.12381195485955

5.44093305813595

3.60454439039680

2.75141055614247

2.13880123277429

2.45920018245199

0.98178937171915

3.21163199785463

3.57700225443644

0.74588039030585

2.09171818613223

1.63968385665961

$-0.75276301219350$

$-1.88358495244005$

$-0.87747603420146$

$-1.18357888031711$

1.59190519755041

$-0.14905362380582$

5.01497600687226

5.48193667379111

1.69305531263421

3.06009018672293

Table SCZ13. Cartesian coordinates for the global minimum ${ }^{4} \mathrm{H}_{5}$ conformer of 7 at the PBE0/DEF2-TZVP/D3BJ/CPCM $\left(\mathrm{CH}_{3} \mathrm{CN}\right) / \mathrm{RIJCOSX}$ level.
C -0.02178419166421
$-0.00072572659194$
$-0.09764077601150$
C -0.31154519513148
0.16389081744308
1.39065971245277
C 0.97331185028332
0.50134472382742
2.12148629742857
C 1.66789901263391
1.58281492592136
1.37113843428259
C 1.32627909804298
1.89859123670294
0.12550204665450
O 0.38980420368873
1.26112232525147
$-0.61194700965194$
1.00386614669728
3.43213040933956
C 0.63810881850253
$-0.39679987402197$
$-0.92370266869492$
$-0.91630378254952$
1.99629538708572
$-1.75731665080349$
$-0.68866693337182$
O -1.59673484096284
C -0.87430848337212
O -0.00308589588400
$-2.67502350032599$
$-1.31353515042581$
$-2.48218289533550$
$-2.11471395268158$
$-3.86599589881432$
$-2.12621885013824$
0.11110242645362
$-0.88977781810521$
1.98114868846017
C -0.48085828459404
C 0.59297006928518
0.75564527649239
4.40893538479748
5.51118821937014
O 0.87997750187358
$-1.05209266494733$
4.34114931851423
O $\quad-1.27043015367555$
$-2.92260461993361$
2.69315124375946
1.41447615879099 


$\begin{array}{llcc}\mathrm{C} & -0.67579558122740 & -4.99782579186956 & -1.45511164610483 \\ \mathrm{C} & -1.72552758150599 & -6.07407049913712 & -1.66539356249016 \\ \mathrm{Cl} & -2.49237500373525 & -6.51341383316063 & -0.12948892685396 \\ \mathrm{Cl} & -0.89888237025701 & -7.48544062302410 & -2.33557401643904 \\ \mathrm{Cl} & -2.96476205246014 & -5.51093290979252 & -2.79564319311397 \\ \mathrm{C} & -0.84465447460780 & -4.26801114067785 & 2.75910102578131 \\ \mathrm{C} & -1.69480917251255 & -4.98260819916314 & 3.79167603180012 \\ \mathrm{Cl} & -1.17565256769895 & -6.67606214711911 & 3.79213856545055 \\ \mathrm{Cl} & -3.41020989018269 & -4.89349915542502 & 3.36528804097968 \\ \mathrm{Cl} & -1.45049967541971 & -4.28647422585382 & 5.39744248690368 \\ \mathrm{C} & -0.13292274033066 & -0.04724032182232 & 6.62482774831122 \\ \mathrm{C} & -1.63882873737859 & -0.01992102703074 & 6.85523049181259 \\ \mathrm{Cl} & -1.97540550632676 & -1.08935181417013 & 8.22488125585629 \\ \mathrm{Cl} & -2.18129804592854 & 1.62265325485853 & 7.23907550211672 \\ \mathrm{Cl} & -2.49619685405318 & -0.59327511827603 & 5.42063537073552 \\ \mathrm{H} & 0.79014442357903 & -0.71575349204037 & -0.25373979444466 \\ \mathrm{H} & -0.99880562000774 & 1.00508761949487 & 1.50375180169602 \\ \mathrm{H} & 1.59798006648529 & -0.38353981551109 & 2.25009759762820 \\ \mathrm{H} & 2.45915528757457 & 2.13313917697070 & 1.86314149410855 \\ \mathrm{H} & 1.78623042923850 & 2.70982353346847 & -0.42607199490959 \\ \mathrm{H} & -2.10657211262608 & 0.18975618394506 & -0.64922629576627 \\ \mathrm{H} & -1.00805136502371 & -0.24452169557525 & -1.97988158889834 \\ \mathrm{H} & -0.21808561088078 & -4.75444385318574 & -2.41365194994481 \\ \mathrm{H} & 0.08870147622391 & -5.37595107886117 & -0.77346492909602 \\ \mathrm{H} & 0.20256722279838 & -4.33424471470415 & 3.05594265317101 \\ \mathrm{H} & -0.98240707837735 & -4.75816236265859 & 1.79372848197649 \\ \mathrm{H} & 0.36355101564362 & 0.36591748725563 & 7.50261893364577 \\ \mathrm{H} & 0.18441688989001 & -1.07725552907084 & 6.46567842409446\end{array}$

Table SCZ14. Cartesian coordinates for the global minimum ${ }^{5} \mathrm{H}_{4}$ conformer of 9 at the PBE0/DEF2-TZVP/D3BJ/CPCM $\left(\mathrm{CH}_{3} \mathrm{CN}\right) / \mathrm{RIJCOSX}$ level.

\begin{tabular}{rrrr}
\hline C & -0.745298 & -0.405026 & -0.867292 \\
C & 0.713676 & -0.621387 & -0.471227 \\
C & 1.646103 & -0.267875 & -1.625121 \\
C & 1.074978 & -0.715604 & -2.921090 \\
C & -0.196323 & -1.091411 & -3.033121 \\
O & -1.114347 & -1.081107 & -2.045068 \\
O & 1.857148 & 1.137518 & -1.665079 \\
C & -1.076007 & 1.078689 & -0.971343 \\
O & 0.950473 & -1.949503 & -0.076155 \\
O & -1.539516 & 1.628765 & -1.932491 \\
C & 2.796873 & 1.582467 & -0.718329 \\
C & 0.701280 & -2.179497 & 1.307802 \\
O & -0.824736 & 1.658405 & 0.196769 \\
C & -1.116978 & 3.063729 & 0.311772 \\
C & -0.702322 & 3.492550 & 1.681182 \\
C & -1.468699 & 3.138965 & 2.788831 \\
C & -1.068396 & 3.503709 & 4.064716 \\
C & 0.101907 & 4.232010 & 4.244553 \\
C & 0.867197 & 4.591180 & 3.144590 \\
C & 0.466348 & 4.220524 & 1.868400 \\
C & 2.869250 & 3.078668 & -0.710977 \\
C & 1.539975 & -1.291493 & 2.183433 \\
C & 0.959874 & -0.259488 & 2.913472 \\
C & 1.746918 & 0.632313 & 3.630070 \\
C & 3.126634 & 0.494246 & 3.625763 \\
C & 3.715857 & -0.540722 & 2.907899 \\
C & 2.926919 & -1.425659 & 2.190006 \\
C & 3.706838 & 3.706228 & 0.208771 \\
C & 3.788186 & 5.087920 & 0.261431 \\
C & 3.028383 & 5.864997 & -0.606189
\end{tabular}




$\begin{array}{rrrr}\mathrm{C} & 2.194155 & 5.245728 & -1.524679 \\ \mathrm{C} & 2.114888 & 3.858541 & -1.578369 \\ \mathrm{H} & -1.387787 & -0.802866 & -0.077277 \\ \mathrm{H} & 0.920220 & 0.060129 & 0.355087 \\ \mathrm{H} & 2.603330 & -0.773172 & -1.442252 \\ \mathrm{H} & 1.711953 & -0.766999 & -3.795010 \\ \mathrm{H} & -0.619636 & -1.473214 & -3.955238 \\ \mathrm{H} & 3.786388 & 1.160341 & -0.947415 \\ \mathrm{H} & 2.536984 & 1.239373 & 0.293156 \\ \mathrm{H} & 0.937750 & -3.232816 & 1.467571 \\ \mathrm{H} & -0.361713 & -2.036047 & 1.537768 \\ \mathrm{H} & -2.186851 & 3.208669 & 0.147033 \\ \mathrm{H} & -0.567480 & 3.603884 & -0.459874 \\ \mathrm{H} & -2.383461 & 2.572081 & 2.648266 \\ \mathrm{H} & -1.671056 & 3.223367 & 4.921401 \\ \mathrm{H} & 0.414835 & 4.518972 & 5.242317 \\ \mathrm{H} & 1.781032 & 5.159117 & 3.279769 \\ \mathrm{H} & 1.070116 & 4.493581 & 1.010230 \\ \mathrm{H} & -0.118315 & -0.136890 & 2.899833 \\ \mathrm{H} & 1.280575 & 1.446155 & 4.173304 \\ \mathrm{H} & 3.745158 & 1.194264 & 4.176339 \\ \mathrm{H} & 4.794510 & -0.652056 & 2.903268 \\ \mathrm{H} & 3.389489 & -2.221036 & 1.614517 \\ \mathrm{H} & 4.293331 & 3.104743 & 0.896845 \\ \mathrm{H} & 4.439852 & 5.561827 & 0.987095 \\ \mathrm{H} & 3.086264 & 6.946688 & -0.562335 \\ \mathrm{H} & 1.595528 & 5.842970 & -2.203789 \\ \mathrm{H} & 1.457823 & 3.376301 & -2.290979\end{array}$

Table SCZ15. Cartesian coordinates for the global minimum ${ }^{5} \mathrm{H}_{4}$ conformer of $\mathbf{1 0}$ at the PBE0/DEF2-TZVP/D3BJ/CPCM $\left(\mathrm{CH}_{3} \mathrm{CN}\right) / \mathrm{RIJCOSX}$ level.

\begin{tabular}{rrrr}
\hline $\mathrm{C}$ & 0.344443 & 0.279026 & 0.020512 \\
$\mathrm{C}$ & -0.055357 & -0.049454 & 1.443186 \\
$\mathrm{C}$ & 1.114794 & -0.493521 & 2.310572 \\
$\mathrm{C}$ & 2.090265 & -1.289401 & 1.524846 \\
$\mathrm{C}$ & 2.007533 & -1.381200 & 0.198341 \\
$\mathrm{O}$ & 1.114766 & -0.755046 & -0.588188 \\
$\mathrm{O}$ & 1.701368 & 0.703289 & 2.872045 \\
$\mathrm{C}$ & 1.116429 & 1.585634 & -0.093287 \\
$\mathrm{O}$ & -0.996963 & -1.123497 & 1.361140 \\
$\mathrm{C}$ & -1.876649 & -1.236567 & 2.369571 \\
$\mathrm{C}$ & -2.789412 & -2.395322 & 2.156106 \\
$\mathrm{O}$ & 0.320265 & 2.616586 & 0.416954 \\
$\mathrm{Si}$ & 0.584293 & 4.254913 & 0.249699 \\
$\mathrm{C}$ & -0.351057 & 4.984233 & 1.713553 \\
$\mathrm{C}$ & 0.043589 & 4.392380 & 3.063446 \\
$\mathrm{C}$ & -0.321735 & 6.509650 & 1.748221 \\
$\mathrm{C}$ & -0.199082 & 4.855135 & -1.361908 \\
$\mathrm{C}$ & 0.432757 & 4.199713 & -2.588422 \\
$\mathrm{C}$ & -1.712647 & 4.658519 & -1.372497 \\
$\mathrm{C}$ & 2.447993 & 4.552150 & 0.184484 \\
$\mathrm{C}$ & 2.825223 & 5.974566 & -0.220764 \\
$\mathrm{C}$ & 3.174879 & 4.134089 & 1.459494 \\
$\mathrm{C}$ & 2.380877 & 0.546199 & 4.015293 \\
$\mathrm{C}$ & 2.954077 & 1.837545 & 4.494672 \\
$\mathrm{O}$ & 2.513674 & -0.519412 & 4.564668 \\
$\mathrm{O}$ & -1.899079 & -0.484837 & 3.310965 \\
$\mathrm{H}$ & -0.565334 & 0.357239 & -0.578125 \\
$\mathrm{H}$ & -0.530173 & 0.824923 & 1.886447 \\
$\mathrm{H}$ & 0.728388 & -1.081108 & 3.144615 \\
$\mathrm{H}$ & 2.852783 & -1.857599 & 2.040542 \\
& & &
\end{tabular}




$\begin{array}{rrrr}\mathrm{H} & 2.667459 & -2.015768 & -0.383018 \\ \mathrm{H} & 1.354468 & 1.730966 & -1.152576 \\ \mathrm{H} & 2.063855 & 1.507488 & 0.450608 \\ \mathrm{H} & -3.523624 & -2.442279 & 2.956845 \\ \mathrm{H} & -2.205910 & -3.318721 & 2.134993 \\ \mathrm{H} & -3.291577 & -2.302286 & 1.191211 \\ \mathrm{H} & -1.387333 & 4.674304 & 1.516037 \\ \mathrm{H} & 0.058742 & 3.300239 & 3.038205 \\ \mathrm{H} & 1.034363 & 4.732762 & 3.374873 \\ \mathrm{H} & -0.662632 & 4.699963 & 3.843110 \\ \mathrm{H} & 0.676979 & 6.883490 & 1.992498 \\ \mathrm{H} & -0.614654 & 6.956780 & 0.794005 \\ \mathrm{H} & -1.004668 & 6.896047 & 2.512992 \\ \mathrm{H} & 0.002801 & 5.933678 & -1.414807 \\ \mathrm{H} & 1.518320 & 4.325769 & -2.622462 \\ \mathrm{H} & 0.223268 & 3.125696 & -2.614707 \\ \mathrm{H} & 0.024956 & 4.626054 & -3.511928 \\ \mathrm{H} & -1.977437 & 3.603756 & -1.244601 \\ \mathrm{H} & -2.209927 & 5.221344 & -0.578519 \\ \mathrm{H} & -2.142669 & 4.988574 & -2.325104 \\ \mathrm{H} & 2.787767 & 3.883274 & -0.620047 \\ \mathrm{H} & 3.911216 & 6.069591 & -0.333014 \\ \mathrm{H} & 2.514945 & 6.703764 & 0.533209 \\ \mathrm{H} & 2.371793 & 6.271545 & -1.169876 \\ \mathrm{H} & 4.259755 & 4.123820 & 1.304957 \\ \mathrm{H} & 2.879429 & 3.138040 & 1.796219 \\ \mathrm{H} & 2.972525 & 4.829297 & 2.278421 \\ \mathrm{H} & 3.269077 & 1.736746 & 5.531066 \\ \mathrm{H} & 3.824369 & 2.086972 & 3.881874 \\ \mathrm{H} & 2.231053 & 2.646637 & 4.393408\end{array}$

Table SCZ16. Cartesian coordinates for the global minimum ${ }^{5} \mathrm{H}_{4}$ conformer of $\mathbf{1 1}$ at the PBE0/DEF2-TZVP/D3BJ/CPCM $\left(\mathrm{CH}_{3} \mathrm{CN}\right) / \mathrm{RIJCOSX}$ level.

\begin{tabular}{lrrr}
\hline $\mathrm{C}$ & 0.484851 & -0.318206 & -1.071805 \\
$\mathrm{C}$ & 1.383220 & -1.434997 & -0.561554 \\
$\mathrm{C}$ & 2.853936 & -1.146226 & -0.861285 \\
$\mathrm{C}$ & 2.989660 & -0.560347 & -2.221206 \\
$\mathrm{C}$ & 1.934039 & -0.125184 & -2.908389 \\
$\mathrm{O}$ & 0.662751 & -0.097187 & -2.473001 \\
$\mathrm{O}$ & 3.440297 & -0.263265 & 0.082559 \\
$\mathrm{C}$ & 0.656483 & 0.976283 & -0.302929 \\
$\mathrm{O}$ & 1.096365 & -2.676177 & -1.169548 \\
$\mathrm{O}$ & -0.423870 & 1.825820 & -0.596761 \\
$\mathrm{Si}$ & -0.908071 & 3.107927 & 0.345756 \\
$\mathrm{C}$ & -0.173321 & -3.235480 & -0.859422 \\
$\mathrm{C}$ & -0.424334 & -3.367158 & 0.614090 \\
$\mathrm{C}$ & 3.721592 & -0.838459 & 1.355659 \\
$\mathrm{C}$ & 2.865038 & -0.269374 & 2.447838 \\
$\mathrm{C}$ & 3.035222 & 1.052254 & 2.864088 \\
$\mathrm{C}$ & 2.245485 & 1.595828 & 3.856605 \\
$\mathrm{C}$ & 1.254183 & 0.823437 & 4.465894 \\
$\mathrm{C}$ & 1.072810 & -0.495810 & 4.067600 \\
$\mathrm{C}$ & 1.878449 & -1.023467 & 3.065271 \\
$\mathrm{C}$ & -1.398807 & -2.599643 & 1.247968 \\
$\mathrm{C}$ & -1.589859 & -2.666949 & 2.614750 \\
$\mathrm{C}$ & -0.794083 & -3.509908 & 3.387499 \\
$\mathrm{C}$ & 0.172579 & -4.301728 & 2.769520 \\
$\mathrm{C}$ & 0.346933 & -4.219514 & 1.395064 \\
$\mathrm{C}$ & -2.579889 & 3.614183 & -0.377661 \\
$\mathrm{C}$ & -2.934317 & 5.068649 & -0.074641 \\
$\mathrm{C}$ & -2.709866 & 3.326673 & -1.871122
\end{tabular}




\begin{tabular}{|c|c|c|c|}
\hline & . & 4.508207 & \\
\hline & & 444 & \\
\hline & 13801 & 35459 & 983 \\
\hline & 074085 & 2.508083 & 275 \\
\hline & 557514 & 597025 & 310 \\
\hline & -1.946246 & 1.262292 & \\
\hline & -1.014209 & -3.482782 & \\
\hline & -0.179499 & -4.275194 & 5.54233 \\
\hline & 22974 & 36367 & \\
\hline & -0.482408 & 681812 & 6.0657 \\
\hline & -0.562035 & -0.611714 & -0.97455 \\
\hline & 1.242156 & -1.505171 & \\
\hline & 3.37 & 9815 & -0.82182 \\
\hline & 3.96 & & \\
\hline & 2.01 & 005 & \\
\hline & 1.60 & 311 & \\
\hline & $0.7 c$ & & \\
\hline & & & \\
\hline & $-0 . c$ & & \\
\hline & & & \\
\hline & 3.6 & & \\
\hline & & & \\
\hline & & & \\
\hline & & & \\
\hline & & & \\
\hline & & & \\
\hline & & & \\
\hline & & & \\
\hline & & & \\
\hline & & & \\
\hline & -2.8 & & \\
\hline & & & \\
\hline & & & \\
\hline & & & \\
\hline & & & \\
\hline & & & \\
\hline & & & \\
\hline & & & \\
\hline & & & \\
\hline & & & \\
\hline & & & \\
\hline & & & \\
\hline & & & \\
\hline & & & \\
\hline & & & \\
\hline & & & \\
\hline & & & \\
\hline & & & \\
\hline & & & \\
\hline & & & \\
\hline & & $-4 .($ & \\
\hline & & & \\
\hline & & & \\
\hline & & & \\
\hline & & & \\
\hline & -0.9600 & 1.354731 & 6.775 \\
\hline
\end{tabular}

Table SCZ17. Cartesian coordinates for the global minimum ${ }^{4} \mathrm{H}_{5}$ conformer of $\mathbf{1 1}$ at the PBE0/DEF2-TZVP/D3BJ/CPCM $\left(\mathrm{CH}_{3} \mathrm{CN}\right) / \mathrm{RIJCOSX}$ level.

\begin{tabular}{rrrr}
\hline $\mathrm{C}$ & 0.65380121953974 & 1.31591689824244 & -0.58333607594035 \\
$\mathrm{C}$ & 0.76143914475567 & 0.70225312045730 & 0.80579815044447
\end{tabular}


C 2.13895775820325

C 2.57566721717256

C 1.91893690868380

O 0.86978595218051

O 2.08062556358990

C -0.68955614861395

O 0.50745005560832

O -1.72374292829470

Si -3.31551809798608

C -0.42338616918663

C -0.63603823999088

C 3.20691876769134

C 3.13241000457178

C 2.22910499424127

C 2.08504214883513

C 2.86248344913449

C 3.78709569520903

C 3.91349857625599

C 0.24022236656783

C 0.04491683377067

C -1.03190229084170

C -1.91853676808709

C -1.71346213547490

C -4.06234138340380

C -3.64343992371307

C -3.76545043606807

C -3.38590093676654

C -4.81226024662827

C -2.62385218372254

C -4.14668747640700

C -5.64667771031197

C -3.48214792774650

O -1.13418127226593

C -2.19185789596864

O 2.78482363275590

C 1.83414364779204

H 1.42562820642569

H 0.01663607218799

H 2.82088647116630

H 3.41830114543597

H 2.17552461822135

H -0.73684109301890

H -0.74147976779722

H -1.37583475967635

H -0.05563197012171

H 3.20348804996144

H 4.13595219834800

H 1.61344385895421

H 1.36345646073148

H 4.39209927796744

H 4.63551894508490

H 1.09254006177139

H 0.73598079045126

H -2.76758385309487

H -2.41595965524145

H $\quad-5.14891273522894$

H -2.56436059133864

H $\quad-3.90971446692154$

H -4.13106050841981

H -2.69165607952255

H $\quad-4.12574494630684$

H $\quad-4.24210544875747$
0.95108093045029

2.34867424600503

3.12043752616686

2.72713880777921

0.75711441350116

1.06588532797129

$-0.67650975102805$

1.39831182518412

1.63391758456661

$-1.18097394777507$

$-2.64429281994681$

0.08534879676428

$-1.40165305221877$

$-2.15057265985104$

$-3.51665953741553$

$-4.16389174637363$

$-3.43141443940509$

$-2.06842265023132$

$-3.40042824903073$

$-4.75796525916845$

$-5.39799508844404$

$-4.65452515424862$

$-3.28921849033912$

0.04229726191136

$-0.24989695529864$

$-1.15321564205007$

3.00831546076204

3.35973895050775

4.24912959323929

2.16052992181572

1.87198074664040

1.58451815006372

$-6.73147825451241$

$-7.41471125890907$

$-5.48376632214015$

$-6.24694746181215$

0.87735981328803

1.18208712349059

0.21162287329116

2.76542317113570

4.15851832807243

0.00718054127704

1.65347288840831

$-0.64408503044611$

$-1.01787095976577$

0.33696375470239

0.47766050201955

$-1.65592486618188$

$-4.06241368707902$

$-3.94527434595196$

$-1.51097095801439$

$-2.91751873959906$

$-5.34575351581711$

$-5.12299386032382$

$-2.72058313902232$

0.20409588842229

$-0.40895890545309$

0.56080921358622

$-1.16032554147734$

$-1.35095206378675$

$-1.00307356186860$

$-2.06269771523510$
1.38816217400439

1.07527191904678

0.21323264675504

$-0.53869815573510$

2.79470217193148

$-1.22166952060696$

0.68896670799861

$-0.33406664951476$

$-0.75097457417174$

1.61693809725280

1.38092753810532

3.34246404975435

3.15889978707036

3.90377482574324

3.71733538322733

2.75962577809065

2.01759274816483

2.21787364563804

0.60827027148237

0.43343133786916

1.04233116227692

1.81722551129835

1.97117592892205

$-1.44608807842843$

$-2.88526230451841$

$-0.54337384076933$

$-2.04344688042264$

$-2.45767629087949$

$-1.58715100504152$

0.86332792070610

0.87125244906632

2.11101583535447

0.83491872273573

1.47474943649055

2.48807289004250

3.20273887494008

$-1.23138106337997$

1.44970407977349

0.95008209082814

1.61422634701582

0.03066747370914

$-1.49463072307666$

$-2.14669441407729$

1.51999498160219

2.63826608119624

4.40582930225726

2.91179091643146

4.64875288696612

4.31019872359544

1.27897661834494

1.62911560258888

0.14848225436688

$-0.15991095529112$

2.29732553922229

2.57343248865054

$-1.44549723763013$

$-2.97089446016041$

$-3.56798267128511$

$-3.25239483146983$

$-0.48409454850036$

0.47743817114587

$-0.92683544207287$ 
H -2.87152540789715

H -5.37793063347168

H $\quad-5.36654160816450$

H -4.81412032424777

H $\quad-3.10101143011072$

H -1.59238569905016

H -2.59413929941471

H -4.01786763758550

H -6.11719802412820

H -5.84464021782386

H -6.16238816149655

H -3.94637846624135

H -2.41565471878834

H -3.58616272969000

H -2.08799746050572

H -2.12752377109324

H -3.16777169483386

H 1.88256764949432

H 2.07283066180605

H 0.82310092690087
2.60627508967717 2.48628879139749 3.81171515335850 4.08619496633601 4.71321694411703 4.01693907932867 5.00529456189912 3.25119208927959 2.29344147449572 0.79585946821555 2.29338680880679 1.99145029974511 1.81395453380524 0.49646560193181 $-8.46321334592492$ $-7.32214499154101$ $-7.05075963901691$ $-7.25474066584779$ $-6.28105779385481$ $-5.85358145570985$
$-2.92718018443506$

$-2.79454910431615$

$-1.62943110883947$

$-3.27818798641210$

$-0.71786302702303$

$-1.30881110401114$

$-2.38000838244667$

0.89884133378247

1.76657205476665

0.87880823083653

0.00450332107645

3.01677732363901

2.14798384452092

2.15788581604486

1.19928912396976

2.56370168764151

1.13807351328055

2.79326373760414

4.27072766304251

3.06803544814380

Table SCZ18. Cartesian coordinates for the global minimum ${ }^{5} \mathrm{H}_{4}$ conformer of 12 at the PBE0/DEF2-TZVP/D3BJ/CPCM $\left(\mathrm{CH}_{3} \mathrm{CN}\right) / \mathrm{RIJCOSX}$ level.
C 1.82821655416207
0.48392630623966
0.49730300283478
C 0.98095311329333
0.65552026312263
1.74022111569518
C 1.59568090267014
1.65604346720535
C 3.07328834325168
1.50038449654294
2.72465599593782
C 3.73045498480375
0.78442228198256
2.76117305587753
0.16242579234093
O 3.18882710419736
O 1.27494355282852
C 1.80284068596665
2.99866179953075
1.71086830046318
1.85072745992420
0.78803441259851
2.38448553574804
O 0.83186169698640
$-0.61326728062041$
O 0.46955371572759
2.01232284031984
2.83407741637487
$-0.40452893947877$
2.34826859818446
$-0.70837557035454$
Si -0.09884363783473
C -0.36380402790701
$-0.74297959108756$
C -1.60536606869115
$-0.68326108944104$
$-2.03691700630189$
3.09032101597182
2.24060635212070
3.36843806572843
2.74125932123551
C -0.29506116636118
4.78500171943284
5.74018320547789
7.05079888107023
2.32890526408098
C 0.71620579543212
C 0.45723165631301
7.42523858852646
6.47830097002782
2.35990544025737
1.98666175261597
$-0.81801290922667$
C -1.83256515443095
C -1.56891378402305
5.16612833966508
1.58389576049921
1.55519282756958
1.92168418065088
$-1.39800843810846$
C -2.83098304590619
$-1.35324043316192$
1.04779321466731
0.27376460015586
0.68487870446715
$-0.59609021054864$
0.12283838541059
0.08265281216492
3.51437719948009
3.97220173055217
2.56425917919362
1.86844125750659
2.63775419347879
$-1.48833452539201$
$-2.66047432125682$
$-0.57972881714701$
$1.64932804185663 \quad-3.50175640762304$
$1.13181885595956 \quad-3.96059835811486$
C -0.24335878098127
C 1.11874493947466
0.48100744708458
$-3.21361996581117$
4.20975839739749
C 1.13252800046978
C 0.86395312038466
C 1.25144930599561
H 1.44091452163247
H 0.01149063972466
H 1.18589590284600
4.92480770201404
$-2.43809640769465$
5.20570514494197
$-3.75878269159859$
$-0.37281341911830$
1.02331858567955
$-1.28772761600052$
$-0.05838579198061$
1.39362867682824
$1.44402858580496 \quad 3.72241128853405$
H 3.63522538846681
1.95153976769774
3.56977445279702 

H 4.80155014538268
H 2.39213298480344
H 2.28189832124734
H -0.42657509937397
H -0.29132704972037
H -0.16455731156703
H -0.77915771503016
H 1.71461319478152
H 1.25666391672726
H -1.01863780932624
H -2.83000067554828
H -2.36122474892070
H -0.83041109070020
H -2.87641561486549
H -4.81791384646422
H -4.69176600135695
H -2.63697120200412
H -1.51353894326909
H -2.12428628127010
H -2.93406416665542
H -3.55385285687438
H -2.88303819666836
H -1.93963171557843
H -3.43460232575915
H -0.67415612479464
H 1.82446467025526
H 1.57554595784823
H 1.01876215314768
H -0.86379731536030
H -2.20995928832393
H -1.20165110456465
H 2.09885203532833
H 1.66817661884242
H -0.06536136782152
H $\quad 0.78589788991560$
H 2.07350004862536
H 1.43034535483855
H $\quad 0.33783441718744$
0.61907240929490
1.47969781606123
2.55285136006111
0.01807749368806
$-1.71437038292235$
3.26015097979768
2.71668619430892
5.44822402136545
7.78353753995795
8.44904301376179
6.75755919802309
4.42589148391935
$-1.98506383743992$
$-1.90926808835743$
$-0.55791625693561$
0.72833041234850
0.65768816780369
4.39926063634472
4.69404950962133
3.12844889349515
4.44950445621592
1.67603272502087
2.21466225992363
3.05540478032924
2.23842815633806
1.93698677849777
0.49142289732848
0.52503480897350
$-0.07768622565178$
0.80868552152668
$-0.22049086571299$
3.69368034303613
5.63263154064286
5.50073958095157
4.23260073148403
5.91029808674860
4.71427906498885
5.79684268795788
1.89953092789522
$-1.29859202610236$
0.10504047437502
3.87849946217986
3.58712314786066
3.82991860419379
2.26602157790457
2.66333707628518
2.00714365236731
1.28821496423788
1.23376783562512
1.87971851202199
0.72246918213525
$-0.65622050799170$
0.07629861428996
2.18890506442015
3.55688642471130
$-0.89058532321488$
$-3.30349830349942$
$-3.28971453765459$
$-2.30231481753234$
$-1.11695416097275$
0.25826142763919
$-0.17161847580313$
$-4.32325948345028$
$-4.18163626449370$
$-3.19959159294247$
$-4.86779075124330$
$-2.32831790768982$
$-3.03970425696090$
$-4.05595684065116$
$-2.53287092305781$
$-3.98997658907925$
$-3.71988254409825$
$-4.60150826675438$
$-1.45917513541197$
$-0.32878491497221$
$-1.17636938604883$

Table SCZ19. Cartesian coordinates for the global minimum ${ }^{4} \mathrm{H}_{5}$ conformer of $\mathbf{1 2}$ at the PBE0/DEF2-TZVP/D3BJ/CPCM $\left(\mathrm{CH}_{3} \mathrm{CN}\right) / \mathrm{RIJCOSX}$ level.

$\begin{array}{llcc}\text { C } & -0.26965319611035 & 0.51356596796620 & 0.19034800974415 \\ \text { C } & -0.27699492470346 & 0.33543194957874 & 1.70477092386832 \\ \text { C } & 1.13040349552071 & 0.04941349281000 & 2.19510890527222 \\ \text { C } & 2.11177962506539 & 0.94355785484922 & 1.50617159917612 \\ \text { C } & 1.78166191954564 & 1.64120943243318 & 0.42372227317109 \\ \text { O } & 0.59173074098985 & 1.58136025033529 & -0.20567245690895 \\ \text { O } & 1.14474732515212 & 0.24223027295806 & 3.60165573660202 \\ \text { C } & -1.63763833132103 & 0.82273553430655 & -0.36572920808557 \\ \text { O } & -1.12475568574577 & -0.74414103025108 & 2.02746095155251 \\ \text { O } & -1.62958058869132 & 0.76384491615257 & -1.76978788002291 \\ \text { Si } & -2.63029941431353 & -0.21301853494918 & -2.67155828210519 \\ \text { C } & -2.00573220547770 & -0.52216372495983 & 3.12208906658079 \\ \text { C } & -3.21842549493435 & 0.26851394436245 & 2.72257983836056 \\ \text { C } & 2.17223023116264 & -0.47262714815056 & 4.26138212687544 \\ \text { C } & 1.95976032181418 & -1.96183807346951 & 4.23957563192087 \\ \text { C } & 0.76747005227655 & -2.50385604085649 & 4.71462607170977 \\ \text { C } & 0.55651580837169 & -3.87279971107107 & 4.69098044007524 \\ \text { C } & 1.53940948180375 & -4.72155748588521 & 4.19307599327287 \\ \text { C } & 2.73030259211148 & -4.19123840695444 & 3.71950227843513 \\ \text { C } & 2.93547368563865 & -2.81672420571170 & 3.74063465677649\end{array}$




\begin{tabular}{|c|c|c|c|}
\hline C & -3.31436534133781 & 1.62820358807987 & 2.99964831315602 \\
\hline C & 733376732834 & | 1061807180 & 0342 \\
\hline C & -5.41953606039051 & 1.74459001299061 & 1.84348809955235 \\
\hline C & -5.33990024361112 & 0.38362016252632 & 1.57360709681388 \\
\hline C & -4.24652006841003 & -0.34688167183798 & 2.01083428450072 \\
\hline 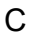 & -2.15020993423054 & -2.03385274512201 & -2.49326371279321 \\
\hline C & -0.68827653239154 & -2.29095557915365 & -2.84884624692412 \\
\hline C & -2.47975270143927 & -2.61496256974434 & -1.11916680652597 \\
\hline C & -2.41165042583706 & 0.40527047519639 & -4.44322681114545 \\
\hline C & -1.00919768948699 & 0.93490801258061 & -4.73237812227767 \\
\hline C & -2.82486185844658 & -0.63228049051760 & -5.48522063042765 \\
\hline C & -4.39783842008853 & 0.03131991448714 & -2.05244664102432 \\
\hline C & -4.80784983514308 & 1.50023164127363 & -2.05413300199629 \\
\hline C & -5.42341847411366 & -0.82585794668664 & -2.78828577463355 \\
\hline $\mathrm{H}$ & 0.10846461145196 & -0.41004270839150 & -0.26784571630904 \\
\hline $\mathrm{H}$ & -0.62814044575014 & 1.26554437159761 & 2.1680537 \\
\hline $\mathrm{H}$ & 1.33889639779501 & -1.00745278965595 & 1.97976397249809 \\
\hline $\mathrm{H}$ & 3.10756549477464 & 1.05806768388639 & 1.91668528377108 \\
\hline $\mathrm{H}$ & 2.45546905815496 & 2.33944636350625 & -0.06117377379736 \\
\hline $\mathrm{H}$ & -1.92200731516743 & 1.83248999674002 & -0.04636313432576 \\
\hline II & -2.35 & 0.126 & 187 \\
\hline $\mathrm{H}$ & 251230001 & -0.0266888 & 5129 \\
\hline $\mathrm{H}$ & -2.30046642604685 & -1.51726043444782 & 3.46097272308203 \\
\hline $\mathrm{H}$ & 2.16370923488295 & -0.10475971923045 & 5.29052407315996 \\
\hline $\mathrm{H}$ & 3.15498587969497 & -0.23325632228488 & 3.83707180 \\
\hline $\mathrm{H}$ & 3679275929 & -1.842 & 5.0 \\
\hline $\mathrm{H}$ & -0.37614901978368 & -4.28257323710237 & 5.06307198664449 \\
\hline $\mathrm{H}$ & 1.37394613689255 & -5.79298552354222 & 4.17370212179075 \\
\hline $\mathrm{H}$ & 3.49923811177976 & -4.84619102849934 & 3.32488464848957 \\
\hline $\mathrm{H}$ & 3.86333474017391 & -2.40207210069228 & 809 \\
\hline $\mathrm{H}$ & -2.52 & 663999 & 3006 \\
\hline $\mathrm{H}$ & -4.46774862600815 & 3.42528130389096 & 2.78049022797190 \\
\hline $\mathrm{H}$ & -6.27278702639645 & 2.31788529469270 & 1.49866832421434 \\
\hline $\mathrm{H}$ & -6.13324275812357 & -0.10724638672967 & 1.02099335602141 \\
\hline $\mathrm{H}$ & -4.18192155280714 & -1.40856879106505 & 3464888227 \\
\hline $\mathrm{H}$ & 0734641234 & -2.56154562407604 & -3.23126692115126 \\
\hline $\mathrm{H}$ & -0.44498987627574 & -3.35542586993835 & -2.75403175046717 \\
\hline $\mathrm{H}$ & -0.45051458770287 & -1.99314226525598 & -3.87281834005065 \\
\hline $\mathrm{H}$ & -0.01197855867932 & -1.74340147522872 & -2.18547184826020 \\
\hline $\mathrm{H}$ & -1.89876125336426 & 4844516 & 1080466 \\
\hline $\mathrm{H}$ & -3.53688896957796 & 307974376 & -0.86429922473137 \\
\hline $\mathrm{H}$ & -2.24795244208246 & -3.68585951136655 & -1.08825548945046 \\
\hline $\mathrm{H}$ & -3.10474738762942 & 1.25398777868886 & -4.52632891001209 \\
\hline $\mathrm{H}$ & -0.25928834661427 & 0.13985739380446 & -4.68506830242554 \\
\hline $\mathrm{H}$ & 23255489 & 1.36 & 2019317 \\
\hline $\mathrm{H}$ & -0.71125491157595 & 1.70575327362296 & -4.01916905839414 \\
\hline $\mathrm{H}$ & -2.15414895753656 & -1.49664385258058 & -5.47649537075034 \\
\hline $\mathrm{H}$ & -2.78666335018053 & -0.20511406754970 & -6.49359530967834 \\
\hline $\mathrm{H}$ & -3.84016402067507 & -1.00552634417745 & -5.32862428759477 \\
\hline $\mathrm{H}$ & -4.37938139161468 & -0.30063629786328 & -1.00601984253870 \\
\hline $\mathrm{H}$ & -5.80357404739702 & 1.62979074116510 & -1.61548735141522 \\
\hline $\mathrm{H}$ & -4.84889107272036 & 1.90359555443896 & -3.07110512950214 \\
\hline $\mathrm{H}$ & -4.11451289574865 & 2.12068601087373 & -1.48079825464960 \\
\hline $\mathrm{H}$ & -5.15509426336534 & -1.88601308227204 & -2.79574395158680 \\
\hline $\mathrm{H}$ & -5.53712070568921 & -0.50779219107988 & -3.82904349746523 \\
\hline-1 & -6.41007548440189 & -0.74156341291034 & -2.31850459321737 \\
\hline
\end{tabular}

Table SCZ20. Cartesian coordinates for the global minimum ${ }^{5} \mathrm{H}_{4}$ conformer of $\mathbf{1 3}$ at the PBE0/DEF2-TZVP/D3BJ/CPCM $\left(\mathrm{CH}_{3} \mathrm{CN}\right) / \mathrm{RIJCOSX}$ level.

$\begin{array}{llll}\text { C } & -0.33204224496426 & 0.71331383924636 & 0.88803627489045 \\ \text { C } & 0.38290905339796 & -0.35036833056616 & 1.70971228247459\end{array}$


C 1.90100847578308

C 2.23867263387321

C 1.32028449017320

O 0.02696131097791

O 2.49297422985021

C -0.10599593511986

O -0.00769238401687

O -0.97581605190582

C -2.22017013278259

C -1.20780602145195

C 2.54828522112905

C 1.54438503759542

C -1.07803341099389

C 0.01580233480337

C 0.13506594483614

C -0.84303717816461

C -1.93432452407475

C -2.04474848804152

C 0.51727180446450

C -0.42935273473772

C -0.35718064917586

C 0.67070438648819

C 1.61574443529844

O -2.61665473022442

C -3.02482449241450

H -1.40406395298555

H 0.12470982922295

H 2.30449335441292

H 3.25124401463798

H 1.54244830267532

H 0.90352182292079

H -0.27341195286518

H -2.03325212594039

H -1.45141811402597

H 3.56100660006898

H 2.40426698163567

H 0.78063920628803

H 0.99330756413323

H -0.74868027284278

H -2.69525571307002

H -2.88981871609831

H 0.44773305791814

H -1.22875649999099

H -1.09898606961217

H 0.73385136211429

H 2.41269206224030

H -3.12686910355666

H -4.00973034233687

H -2.51342367554582
$-0.26902631124220$

$-0.00748316510534$

0.39187665377912

0.64905048029589

0.74289270714042

2.11018827085915

$-1.64193602216594$

3.05198891935675

3.13707692679633

$-2.09597669012710$

0.46029758733586

1.25935736017618

$-2.34349806934140$

$-3.04577693756177$

$-3.28479639925424$

$-2.82925811280609$

$-2.12842195352457$

$-1.88113236603063$

0.63922165538979

1.39227937596494

2.77687285634471

3.40604277157047

2.65114399686485

2.46966994701001

4.14654403350644

0.50959421134089

$-0.19961851813792$

$-1.24345705417485$

$-0.17412577535849$

0.53455579742904

2.45653086077119

2.12666954766231

$-1.39774477809359$

$-3.02649280055943$

0.71536213011139

$-0.61024821835265$

$-3.39949102626542$

$-3.82864196493345$

$-3.01431391518873$

$-1.75962217930866$

$-1.31544989967196$

$-0.44384290249103$

0.89402405800515

3.36680310697643

4.48857434163578

3.14547698609461

3.83043528793627

4.24553476408592

5.11099591657536
1.54461131442807

0.12106689657584

$-0.75740449147364$

$-0.49307103685481$

2.34563990918955

1.42687214408290

1.29185170747918

0.79187133700960

1.27825575576274

1.88508882866479

3.73928760730308

4.52129331456341

3.36400871992162

3.86571535352933

5.22589529874509

6.10313461497506

5.61130881827635

4.24875981643118

5.22338042498491

5.90591093883099

5.89145231368018

5.19837975664342

4.52197642578356

2.20182485645745

0.53028193877675

0.90490852138267

2.76250552213292

1.85021472950220

$-0.22455939869800$

$-1.80962622825433$

1.21564867368396

2.50359283987517

1.69924459157550

1.36576157850873

4.06684469147477

3.91971883979019

3.18269252428566

5.60484189906981

7.16726024723784

6.29002472075346

3.86919893575486

5.22860665768045

6.44279431014651

6.41810787180769

5.18566937632718

3.97602041594338

$-0.51060046670833$

0.98064791679491

0.53170710880116

Table SCZ21. Cartesian coordinates for the global minimum ${ }^{4} \mathrm{H}_{5}$ conformer of $\mathbf{1 3}$ at the PBE0/DEF2-TZVP/D3BJ/CPCM $\left(\mathrm{CH}_{3} \mathrm{CN}\right) / \mathrm{RIJCOSX}$ level.

\begin{tabular}{rrrr}
\hline C & -0.086942 & 0.251421 & 0.160060 \\
C & 0.157210 & 0.140656 & 1.660404 \\
C & 1.649331 & 0.141240 & 1.935211 \\
C & 2.321908 & 1.169711 & 1.079772 \\
C & 1.698638 & 1.763773 & 0.066719 \\
O & 0.454448 & 1.461425 & -0.363523 \\
O & 1.846585 & 0.421894 & 3.313294 \\
C & -1.541334 & 0.190367 & -0.210627 \\
O & -0.442063 & -1.053775 & 2.099623
\end{tabular}




$\begin{array}{rrrr}\text { O } & -2.247480 & 1.186393 & 0.529641 \\ \mathrm{C} & -3.581898 & 1.109457 & 0.510969 \\ \mathrm{C} & -1.073385 & -0.964946 & 3.354427 \\ \mathrm{C} & 3.016386 & -0.170210 & 3.855732 \\ \mathrm{C} & 2.855569 & -1.646792 & 4.085793 \\ \mathrm{C} & -2.133488 & -2.327824 & 3.839818 \\ \mathrm{C} & -2.479411 & -2.441626 & 5.055615 \\ \mathrm{C} & -2.161460 & -4.835617 & 5.557870 \\ \mathrm{C} & -1.496481 & -4.729248 & 4.847014 \\ \mathrm{C} & -1.147031 & -3.481283 & 3.635417 \\ \mathrm{C} & 3.574471 & -2.575498 & 3.341962 \\ \mathrm{C} & 3.387200 & -3.938585 & 3.539747 \\ \mathrm{C} & 2.472443 & -4.383008 & 4.482630 \\ \mathrm{C} & 1.750626 & -3.461585 & 5.232032 \\ \mathrm{C} & 1.945602 & -2.104997 & 5.036427 \\ \mathrm{O} & -4.184811 & 0.265140 & -0.104145 \\ \mathrm{C} & -4.196961 & 2.184422 & 1.343093 \\ \mathrm{H} & 0.409769 & -0.590582 & -0.341465 \\ \mathrm{H} & -0.293101 & 1.008827 & 2.155493 \\ \mathrm{H} & 2.023050 & -0.867692 & 1.716625 \\ \mathrm{H} & 3.330322 & 1.479880 & 1.325928 \\ \mathrm{H} & 2.141840 & 2.559718 & -0.521412 \\ \mathrm{H} & -1.937636 & -0.797263 & 0.021206 \\ \mathrm{H} & -1.661725 & 0.374602 & -1.280443 \\ \mathrm{H} & -1.966869 & -0.327459 & 3.281869 \\ \mathrm{H} & -0.401288 & -0.499291 & 4.083220 \\ \mathrm{H} & 3.189263 & 0.345347 & 4.803221 \\ \mathrm{H} & 3.883650 & 0.019909 & 3.212094 \\ \mathrm{H} & -2.381526 & -1.545156 & 5.616273 \\ \mathrm{H} & -2.999227 & -3.757842 & 6.507035 \\ \mathrm{H} & -2.430918 & -5.812253 & 5.238413 \\ \mathrm{H} & -1.239211 & -5.622124 & 3.075811 \\ \mathrm{H} & -0.612341 & -3.400479 & 2.196924 \\ \mathrm{H} & 4.287418 & -2.228959 & 2.600197 \\ \mathrm{H} & 3.953619 & -4.652486 & 2.951785 \\ \mathrm{H} & 2.317928 & -5.445696 & 4.633188 \\ \mathrm{H} & 1.028604 & -3.804016 & 5.964596 \\ \mathrm{H} & 1.379647 & -1.387788 & 5.621922 \\ \mathrm{H} & -3.896712 & 2.058503 & 2.386052 \\ \mathrm{H} & -3.838420 & 3.162194 & 1.015454 \\ \mathrm{H} & -5.280767 & 2.139849 & 1.266759 \\ & & & \end{array}$

Table SCZ22. Cartesian coordinates for the global minimum ${ }^{5} \mathrm{H}_{4}$ conformer of $\mathbf{1 4}$ at the PBE0/DEF2-TZVP/D3BJ/CPCM $\left(\mathrm{CH}_{3} \mathrm{CN}\right) / \mathrm{RIJCOSX}$ level.

\begin{tabular}{llll}
\hline $\mathrm{C}$ & 0.68266349554782 & 0.83655078765648 & 0.59449437355710 \\
$\mathrm{C}$ & 1.004752407449298 & -0.08754096047194 & 1.76058975510449 \\
$\mathrm{C}$ & 2.27711948129147 & -0.90067500840059 & 1.51450135725450 \\
$\mathrm{C}$ & 2.31730944820690 & -1.35811812905264 & 0.10147709320083 \\
$\mathrm{C}$ & 1.49760372011662 & -0.85778681726257 & -0.82238290647235 \\
$\mathrm{O}$ & 0.59289989129746 & 0.11783258025275 & -0.63830374021540 \\
$\mathrm{O}$ & 3.46742961035271 & -0.17886195411891 & 1.79511185359475 \\
$\mathrm{C}$ & 1.65158827179814 & 1.98907483195892 & 0.47115841061335 \\
$\mathrm{O}$ & -0.04214574403111 & -1.01748120170310 & 1.95738343694158 \\
$\mathrm{O}$ & 1.11852623920736 & 2.93322230750736 & -0.42289111610563 \\
$\mathrm{C}$ & 1.973111140498835 & 4.03651396724152 & -0.57750622398615 \\
$\mathrm{C}$ & -1.09855981128988 & -0.52536810663413 & 2.75555907222178 \\
$\mathrm{C}$ & -0.70464650854586 & -0.31268443411856 & 4.19313289634299 \\
$\mathrm{C}$ & 3.73337969917265 & 0.03860527591183 & 3.17491827942025 \\
$\mathrm{C}$ & 3.47746905280205 & 1.46038461203251 & 3.58901545054529 \\
$\mathrm{C}$ & 2.51294661545181 & 1.77214358271859 & 4.54035009874346
\end{tabular}



C 2.26171952704263
C 2.97518932314490
C 3.94620380806318
C 4.19620073113373
C 0.04507688079205
C 0.39747515711961
C -0.00141159076822
C -0.74681294949929
C -1.08904393820439
H -0.31512076277433
H 1.13710554549730
H 2.22716605499387
H 3.00577031984289
H 1.47982892646654
H 2.63254790029407
H 1.79385096913292
H 2.95322630857657
H 1.50508348266547
H 2.13079604398330
H -1.88772387740414
H -1.50687853081111
H 4.78925762003133
H 3.14413084490286
H 1.94325836452738
H 1.50221412721194
H 2.77727344743143
H 4.50819508692642
H 4.94910030960762
H 0.35917217448201
H 0.98519470040934
H 0.27572528236534
H -1.05124780479790
H -1.65626349175475
3.09339126411377
4.11797021574445
3.81515240705863
2.49560592226787
$-1.27351713025057$
$-1.08661293301601$
0.06269547138401
1.02474952592197
0.83884982712087
1.25788445655649
0.52520936349479
$-1.76970363404872$
$-2.14379111380680$
$-1.22421672832090$
1.63818832418284
2.44042647595811
3.73512002547860
4.72379816088230
4.56068565468018
$-1.27904101737489$
0.40641386127095
$-0.20562395731339$
$-0.64724191332777$
0.97563013351834
3.32088072584412
5.15038741615729
4.61191273705227
2.26330283186250
$-2.16933408231494$
$-1.83962102656317$
0.20933045463005
1.92954649280847
1.60160245483075
4.88806347680579
4.28363536813276
3.33566965154272
2.99444114470140
4.86812018153111
6.19590147580603
6.86924855769129
6.20347841389942
4.87003874634558
0.73930828004919
2.65761700991770
2.18434144688146
$-0.18389020214867$
$-1.84360094670605$
0.12665126102219
1.46287353551133
$-0.97173410423059$
$-1.28364810140114$
0.37526507801177
2.68817858086300
2.34535646602823
3.32933432891843
3.79299271171682
5.00804352835435
5.62779234433660
4.54940179097521
2.86098016434540
2.24840240082704
4.34353536331180
6.70924144349167
7.90710374528333
6.71767280726549
4.34591096015854

Table SCZ23. Cartesian coordinates for the global minimum ${ }^{4} \mathrm{H}_{5}$ conformer of $\mathbf{1 4}$ at the PBE0/DEF2-TZVP/D3BJ/CPCM $\left(\mathrm{CH}_{3} \mathrm{CN}\right) / \mathrm{RIJCOSX}$ level.
C -0.06855726474791
$-0.55941470494227$
0.34165466620310
C -0.01074693561855
0.00521530196321
C 1.39718231771177
$-0.10658504198400$
1.75551302432353
C 2.38484895844107
0.27999539202096
2.30423995801380
C 2.03402843416826
0.41474875014312
0.14840181067493
0.74723528944149
1.24828576730915
$-0.02777728092166$
O 0.81172022208105
O 1.49345486480647
C -1.45158006108137
$-0.51620131705784$
$-0.53232195167675$
3.43568056666152
O -0.90600897825003
$-0.72589827674853$
$-0.25035423185601$
2.55861919883868
0.77920600957308
0.84239684804638
$-0.15111865035273$
$-0.61309122230879$
0.06320100482221
3.33490437655521
C -1.77285271745854
C -2.55462518583265
$-0.80167400931873$
0.29755781292385
C 2.41666762325813
C 1.91681490451287
$-0.89693654401254$
$-2.07789349112079$
$-3.17797604205180$
$-3.10635073224367$
$-1.93018275351560$
$-0.83416015427151$
4.27654252157758
4.40944835807060
5.17541703472271
5.22472946517685
5.92683444304623
6.57905715289405
6.53076302820022
5.83803899244971
4.15238387233555
5.04723040456091
6.07570582974065
6.20496768191722
5.31214886473354 
H 0.25189906093181

H -0.29163533984231

H 1.54425266284931

H 3.40480814697340

H 2.71745531461505

H -2.08270373781677

H -1.39889460170920

H $\quad-3.96391606749995$

H -3.65053865292584

H -3.37573994571915

H -2.46034073968477

H -1.20297987355184

H 2.56265315561894

H 3.38890681704343

H 3.59875664251105

H 2.74881376324349

H 0.56707677395875

H $\quad-0.75365858239080$

H 0.11325616123011

H -2.01808410573779

H -3.32155534637504

H -4.58932564663265

H -4.54434123719747

H $\quad-3.24265168171709$
$-1.61006963385425$ 1.06299664878020 $-1.14537853155377$ 0.50569986215169 0.75727268547001 $-1.23517748472953$ $-0.83651108775571$ 0.17537536218201 1.86986106653799 0.56900953114085 0.62068577865149 0.80340216195335 1.14516049895464 0.06966629520487 $-2.13917122873910$ $-4.09419921064186$ $-3.96589966685911$ $-1.87132240752663$ 0.08100912088439 $-2.65850711095636$ $-4.03630102217314$ $-2.95826259521796$ $-0.48988813361739$ 0.87730706148313
0.36628813145061

1.72079515030818

2.62866015296972

1.53546288096475

$-0.79728194674428$

0.28745251848044

$-1.30073607245185$

$-0.04026073699306$

$-0.49069379291161$

$-1.67466442310128$

2.68129359364584

3.90842774616756

5.08407197486324

3.95532524276358

4.70407047511102

5.95584811118445

7.12009858099401

7.02919743979688

5.79749465378948

3.35964945900891

4.94145516577742

6.77628260620746

7.00869516144004

5.42441605706358

Table SCZ24. Cartesian coordinates for the global minimum ${ }^{5} \mathrm{H}_{4}$ conformer of $\mathbf{1 5}$ at the PBE0/DEF2-TZVP/D3BJ/CPCM $\left(\mathrm{CH}_{3} \mathrm{CN}\right) / \mathrm{RIJCOSX}$ level.

$\begin{array}{llcc}\text { C } & 0.25158072281910 & -0.36240971930055 & -0.45930145629017 \\ \text { C } & -0.33027706248777 & -0.33933539822418 & 0.94494514549180 \\ \text { C } & 0.70225696742599 & 0.09177634487586 & 1.98633654111731 \\ \text { C } & 2.04107976164206 & -0.45937276113361 & 1.65241659393172 \\ \text { C } & 2.29069034511116 & -1.04654832988361 & 0.48360191863891 \\ \text { O } & 1.43002977719355 & -1.15787824846770 & -0.54878587883476 \\ \text { O } & 0.77229788206881 & 1.50984636959156 & 2.08283559618127 \\ \text { C } & 0.60568320186980 & 1.02010086130130 & -0.98517080545886 \\ \text { O } & -0.82362860976522 & -1.61740563964303 & 1.28860994362944 \\ \text { O } & -0.56048927319592 & 1.83563503835559 & -0.85169070347327 \\ \text { C } & -0.35397551563613 & 3.13732063618224 & -0.75573349970485 \\ \text { C } & -2.23444892271105 & -1.72579782574911 & 1.16335336887309 \\ \text { C } & -0.26244391481691 & 2.05282208875939 & 2.86797855075609 \\ \text { C } & -0.16478488930969 & 3.54763040421131 & 2.91674071082968 \\ \text { C } & -2.95958155053102 & -0.78595495795512 & 2.08706091991016 \\ \text { C } & -2.77611816824165 & -0.88208686769823 & 3.46525648777867 \\ \text { C } & -3.38433499521009 & 0.02150247213534 & 4.32161797943158 \\ \text { C } & -4.18892040968635 & 1.03392808147400 & 3.81035812321676 \\ \text { C } & -4.38205858614928 & 1.13374799119323 & 2.44096445252363 \\ \text { C } & -3.76666834711954 & 0.22877725746677 & 1.58493193418901 \\ \text { C } & 0.96397089786036 & 4.22975366151473 & 2.47912761062312 \\ \text { C } & 1.02068554722204 & 5.61637471342654 & 2.55640826492760 \\ \text { C } & -0.04809261494121 & 6.33457980623804 & 3.07228215695811 \\ \text { C } & -1.18112612004097 & 5.65713239878865 & 3.50853415922952 \\ \text { C } & -1.23827939617285 & 4.27510369764604 & 3.42692538801890 \\ \text { O } & -1.52378582965025 & 3.70217911137571 & -0.45109931380427 \\ \text { O } & 0.68374031826820 & 3.71686979940719 & -0.92057509920031 \\ \text { C } & -1.48471252080584 & 5.09654615384738 & -0.23363829540507 \\ \text { C } & -2.90225639029932 & 5.62645730318301 & -0.32575191865370 \\ \text { Cl } & -2.82340742841867 & 7.36208478135497 & 0.01368159091138 \\ \text { Cl } & -3.95647735957957 & 4.84073641585063 & 0.85637685794069 \\ \text { Cl } & -3.55742603421990 & 5.36908797924109 & -1.95202841901566 \\ \text { H } & -0.46908162414991 & -0.83602407805129 & -1.12924427916454 \\ \text { H } & -1.14155346457644 & 0.39093053028464 & 0.94731784968735\end{array}$


H 0.37243329148052

H 2.82665713885175

H 3.24057616313909

H 0.88730042431410

H 1.42446478253001

H -2.46626150161586

H -2.54675861972696

H -0.20201782223249

H -1.25008434833990

H -2.14120828877384

H -3.23138188933754

H -4.66108798019285

H $\quad-5.00624167177650$

H $\quad-3.90498557169967$

H 1.79440328616712

H 1.90407002583388

H $\quad-0.00550443218932$

H -2.02828935874142

H -2.13106776209946

H -1.09224515371879

H -0.87250810563740
$-0.31104021233520$

$-0.43364027344345$

$-1.51693408851479$

0.96100319022205

1.45727987719870

$-2.76563253152846$

$-1.54386470436022$

1.64554299092348

1.77288292368101

$-1.66571208587407$

$-0.06090316695186$

1.74395139445443

1.92155758837179

0.31857008205985

3.67294546941235

6.13674331534782

7.41655597690319

6.20903821796219

3.75206317672450

5.31335816668735

5.59709062146057
2.95295320769815

2.39734294643919

0.25450042930731

$-2.03772289900746$

$-0.41564573657983$

1.40343669412417

0.12726782552327

3.88831838716117

2.47776381790487

3.86555104626215

5.39201129356973

4.48012676036867

2.03491040247008

0.51206645393971

2.06493867149581

2.20297145615506

3.12522196697895

3.89995708952605

3.75510812017860

0.76215906967177

$-0.98461347897776$

Table SCZ25. Cartesian coordinates for the global minimum ${ }^{4} \mathrm{H}_{5}$ conformer of $\mathbf{1 5}$ at the PBE0/DEF2-TZVP/D3BJ/CPCM $\left(\mathrm{CH}_{3} \mathrm{CN}\right) /$ RIJCOSX level.

\begin{tabular}{llcc}
\hline $\mathrm{C}$ & 0.25762434133135 & -0.51589923520203 & -0.64697450860285 \\
$\mathrm{C}$ & 0.10888394312456 & -0.26802939583999 & 0.85041632825558 \\
$\mathrm{C}$ & 1.39030990083289 & 0.31403054447605 & 1.42576774053881 \\
$\mathrm{C}$ & 1.97495416074032 & 1.32048863600911 & 0.48684417135878 \\
$\mathrm{C}$ & 1.55769354215478 & 1.44084494087125 & -0.76859973797830 \\
$\mathrm{O}$ & 0.63756190843338 & 0.65461520483812 & -1.36280454376658 \\
$\mathrm{O}$ & 1.05970227358417 & 0.90401085855608 & 2.67354822730280 \\
$\mathrm{C}$ & -1.00175862080220 & -1.02916964664584 & -1.28627991914411 \\
$\mathrm{O}$ & -0.19584407923551 & -1.51929952309403 & 1.42303890106620 \\
$\mathrm{O}$ & -2.02802645435351 & -0.05227779604801 & -1.08174778470262 \\
$\mathrm{C}$ & -3.22174148478606 & -0.37471772973477 & -1.53654439778920 \\
$\mathrm{C}$ & -0.98320056046239 & -1.47777086448761 & 2.60764794258507 \\
$\mathrm{C}$ & 2.15114579716904 & 1.02728314983937 & 3.54809638038467 \\
$\mathrm{C}$ & 1.71683065718007 & 1.67905882031538 & 4.82687622394507 \\
$\mathrm{C}$ & -2.41828519077933 & -1.11974979359920 & 2.34639496912607 \\
$\mathrm{C}$ & -2.96047954886533 & 0.06345780766392 & 2.83529701541574 \\
$\mathrm{C}$ & -4.28939944370327 & 0.38776879580616 & 2.59367362076084 \\
$\mathrm{C}$ & -5.08941080585353 & -0.47287777105705 & 1.85786821665188 \\
$\mathrm{C}$ & -4.55570103052479 & -1.65578360676294 & 1.35979294024836 \\
$\mathrm{C}$ & -3.22911314195070 & -1.97467372770324 & 1.60268715802702 \\
$\mathrm{C}$ & 2.56188358893346 & 1.63427684129551 & 5.93359712090405 \\
$\mathrm{C}$ & 2.20182393625569 & 2.24899508576376 & 7.12258534161073 \\
$\mathrm{C}$ & 0.98582810305399 & 2.91477338097131 & 7.22384918256555 \\
$\mathrm{C}$ & 0.13855670644724 & 2.95956953884966 & 6.12666138183948 \\
$\mathrm{C}$ & 0.50216832119752 & 2.34663567154804 & 4.93354857701181 \\
$\mathrm{O}$ & -4.03522399351983 & 0.65854383057742 & -1.30447495101523 \\
$\mathrm{O}$ & -3.53429190580165 & -1.39945956257395 & -2.08100930335634 \\
$\mathrm{C}$ & -5.36748266908724 & 0.48109330511251 & -1.73146108752780 \\
$\mathrm{C}$ & -6.03001913802403 & 1.84460037175793 & -1.78752817615099 \\
$\mathrm{Cl}$ & -7.70289283493311 & 1.58202849829943 & -2.30466108175818 \\
$\mathrm{Cl}$ & -5.20627530335792 & 2.88928565091897 & -2.95566579767767 \\
$\mathrm{Cl}$ & -6.02033054963386 & 2.62464338959433 & -0.19985894113518 \\
$\mathrm{H}$ & 1.03144982585733 & -1.28109832918223 & -0.79493692465712 \\
$\mathrm{H}$ & -0.70285761120497 & 0.44997322843689 & 1.01581619828186 \\
$\mathrm{H}$ & 2.08937185190248 & -0.51620954313761 & 1.60295375136138 \\
$\mathrm{H}$ & 2.73417999418819 & 2.00325024641521 & 0.84785965150064 \\
$\mathrm{H}$ & 1.92759452976822 & 2.20069216836571 & -1.44796584813188
\end{tabular}




$\begin{array}{ll}\mathrm{H} & -1.29243554737841 \\ \mathrm{H} & -0.84769625660796 \\ \mathrm{H} & -0.54643095815698 \\ \mathrm{H} & -0.91796723918609 \\ \mathrm{H} & 2.95261214297330 \\ \mathrm{H} & 2.57796113225504 \\ \mathrm{H} & -2.33693666593123 \\ \mathrm{H} & -4.69797406687972 \\ \mathrm{H} & -6.12825896358900 \\ \mathrm{H} & -5.17773375297638 \\ \mathrm{H} & -2.81615206555939 \\ \mathrm{H} & 3.50976072039987 \\ \mathrm{H} & 2.86998164804141 \\ \mathrm{H} & 0.70163807265277 \\ \mathrm{H} & -0.81321723459649 \\ \mathrm{H} & -0.16042211580592 \\ \mathrm{H} & -5.40596195864516 \\ \mathrm{H} & -5.91729490628511\end{array}$

$-1.97666237128310$ $-1.17045580489464$ $-0.78591570529505$ $-2.48742836972602$ 1.62594834700691 0.03715935423337 0.73640003888599 1.31496340958454 $-0.22477736130651$ $-2.33232465694348$ $-2.90119179538022$ 1.10943001629486 2.20412242637880 3.39320183342124 3.47503662855601 2.38449901157801 0.03223040629810 $-0.14824784862243$
$-0.83551327697006$ $-2.35735536889392$ 3.33174909966862

3.01928185364160 3.09160568785607 3.76200498002156

3.41522803873674 2.97969871679199 1.67164812265650

0.78435850845555

1.21668836272605 5.86259380198922

7.97553829702895

8.15436788730981

6.19581874772699

4.07801539396368

$-2.72464241493858$

$-1.02868547511911$

Table SCZ26. Cartesian coordinates for cycloaddition transition state of the ${ }^{5} \mathrm{H}_{4}$ conformer of 1 with TCAI at the PBE0/DEF2-SVP/D3BJ/CPCM $\left(\mathrm{CH}_{3} \mathrm{CN}\right) / \mathrm{RIJCOSX}$ level.

\begin{tabular}{|c|c|c|c|}
\hline $\mathrm{C}$ & .26047249 & & \\
\hline C & 04935328851590 & 0.44500504866270 & 88516 \\
\hline C & 1.55203113381240 & 387887787 & 0616 \\
\hline C & 2.23437339099628 & -0.39917414745973 & 186413734 \\
\hline C & 69507885173916 & -0.35605728375687 & 33101185 \\
\hline $\mathrm{O}$ & 336 & 5836 & 392 \\
\hline $\mathrm{O}$ & 2.1142553515 & 68792 & 595 \\
\hline C & -0.02898396446596 & 633701310 & 6202 \\
\hline $\mathrm{O}$ & -0.46754681901294 & -0.84315905129894 & 6909 \\
\hline $\mathrm{O}$ & -0.40693212582423 & 1387 & 123 \\
\hline C & 88674 & & 43 \\
\hline $\mathrm{O}$ & 1.7 & 221 & 260 \\
\hline C & 31800260208 & 1717 & -3.79 \\
\hline C & -1.76731600283794 & 67998 & 544 \\
\hline C & 74 & 2.3 & 403 \\
\hline C & 76 & 777 & 99 \\
\hline $\mathrm{O}$ & 1.5 & 442 & 097 \\
\hline C & 9246 & 37810 & 7764 \\
\hline $\mathrm{O}$ & 9036 & 39058 & 070 \\
\hline $\mathrm{H}$ & -1.3 & 5644 & 319 \\
\hline $\mathrm{H}$ & -0.4 & 2682 & 042 \\
\hline $\mathrm{H}$ & 486 & 5519 & 7276 \\
\hline $\mathrm{H}$ & 3.3 & -0.5 & 2826 \\
\hline $\mathrm{H}$ & 2.21 & -0.7 & -1.3 \\
\hline $\mathrm{H}$ & 1.02 & 2.5 & -0. \\
\hline $\mathrm{H}$ & -0.6 & 3831 & 0835 \\
\hline $\mathrm{H}$ & -0.6 & 226 & 9345 \\
\hline $\mathrm{H}$ & 0.81240276827487 & 3.04 & -4.512 \\
\hline $\mathrm{H}$ & -0.6 & 4618 & 369 \\
\hline $\mathrm{H}$ & & & \\
\hline $\mathrm{H}$ & 3.6 & 15 & 319 \\
\hline $\mathrm{H}$ & 2.02146767361599 & 25708 & 6071 \\
\hline $\mathrm{H}$ & -1.36198806118780 & 7599 & 53220 \\
\hline $\mathrm{H}$ & -2.3 & -2.8 & 5389 \\
\hline $\mathrm{H}$ & -3.0 & 9599 & 2181 \\
\hline C & 32330 & 3479 & 9341 \\
\hline $\mathrm{O}$ & 2.25355607412968 & 49705834106 & 2.237108 \\
\hline $\mathrm{N}$ & 61115693229 & -2.83153302 & 089 \\
\hline C & 0.39 & -3.96 & 0.38774 \\
\hline C & 46887528559 & 1899 & 3033 \\
\hline
\end{tabular}


O $\quad 0.26206632762911$

Cl -1.50820194705897

Cl -1.30291712365475

Cl 0.76722271965626
$-4.61876563216019$

$-3.01190480236852$

$-5.82975505843439$

$-4.55490238652613$
1.39339365358122

$-1.29736957678028$

$-0.67097941342304$

$-2.24350074448383$

Table SCZ27. Cartesian coordinates for cycloaddition transition state of the ${ }^{4} \mathrm{H}_{5}$ conformer of 1 with TCAI at the PBE0/DEF2-SVP/D3BJ/CPCM $\left(\mathrm{CH}_{3} \mathrm{CN}\right) / \mathrm{RIJCOSX}$ level.

\begin{tabular}{|c|c|c|c|}
\hline C & -0.81352855027823 & 0.89127223726282 & -0.53863460965642 \\
\hline C & -0.60260121855877 & 0.47384315908663 & 0.91132384297537 \\
\hline C & 0.85132961567942 & 0.65215164121883 & 1.33624092230012 \\
\hline C & 1.39958642154785 & 1.95739383207658 & 0.82241772409782 \\
\hline C & 0.67341677784128 & 2.68708722016791 & -0.09577394266659 \\
\hline $\mathrm{O}$ & -0.32705095403215 & 2.22608439802522 & $-0.783742 \varepsilon$ \\
\hline $\mathrm{O}$ & 0.95016876219561 & 0.68278245641076 & 2.75421882739205 \\
\hline C & -2.27222075208432 & 0.87584499874926 & -0.93046788226230 \\
\hline $\mathrm{O}$ & -0.96519169984350 & -0.89505355310373 & 0.95857887575760 \\
\hline $\mathrm{O}$ & -2.41894837316704 & 1.06123463425505 & -2.32807174687699 \\
\hline C & -2.3682107 & -0.04140369986191 & -3.09671 \\
\hline $\mathrm{O}$ & 33330 & -1.15023040589114 & -2.6398 \\
\hline C & -2.4928572 & 0.30226463679478 & -4.54507181663327 \\
\hline C & -1.5634691 & -1.37234273824745 & 2.067996496 \\
\hline C & 1.25518242535338 & -0.46241223750271 & 3.392395 \\
\hline C & 1.2605 & 4963002 & $4.8715 \xi$ \\
\hline $\mathrm{O}$ & 1.463 & -1.501450489 & 32 \\
\hline C & 85916 & -2.84464649347771 & 1.95993 \\
\hline $\mathrm{O}$ & 4513501190 & -0.677750151 & 511 \\
\hline $\mathrm{H}$ & 00540 & 0.2205088 & -1.1 \\
\hline $\mathrm{H}$ & -1.24 & 1.059427432 & 529 \\
\hline $\mathrm{H}$ & 1.4223 & -0.20582549820951 & 0.951506 \\
\hline $\mathrm{H}$ & 2.04 & 52017902 & 272 \\
\hline $\mathrm{H}$ & 0.94216321037933 & 3.71315692790046 & -0.368255 \\
\hline $\mathrm{H}$ & -2.801 & 1.70627647 & -0.4 \\
\hline $\mathrm{H}$ & -2.712 & $-0.081194394 C$ & -0.62245 \\
\hline $\mathrm{H}$ & -3.47099209025407 & 0.7711118894 & -4.72931944367155 \\
\hline $\mathrm{H}$ & 871150482 & 1.03670600911094 & 97788929 \\
\hline $\mathrm{H}$ & 254780566 & -0.60286272660362 & -5.15311772923183 \\
\hline $\mathrm{H}$ & 42029 & -0.1676768714 & 5.20 \\
\hline $\mathrm{H}$ & 1.72026858256442 & -1.12974102175085 & 58887988 \\
\hline $\mathrm{H}$ & 1.78467553147167 & 0.66240428396069 & 14721807 \\
\hline $\mathrm{H}$ & -2.13845217047135 & -3.11415813888452 & 0.95896309460325 \\
\hline $\mathrm{H}$ & 74005 & -3.3350624 & 987 \\
\hline $\mathrm{H}$ & 05649 & -3.181 & 586 \\
\hline C & 2.81244720 & 1.5184901626 & -0.55662228765901 \\
\hline $\mathrm{O}$ & 3.73824807911170 & 2.23442497890487 & -0.40880833058530 \\
\hline $\mathrm{N}$ & 2.28263146167108 & 0.54559284119632 & -1.20025212364060 \\
\hline C & 3.05242945 & -0.29000825788259 & -1.95492794480831 \\
\hline C & 2.15122014373456 & -1.26681213464300 & -2.77675505835768 \\
\hline $\mathrm{O}$ & 4.25336074127357 & -0.35395248570634 & -2.02498595373705 \\
\hline $\mathrm{Cl}$ & 1.14393216925627 & -2.20410778609258 & -1.64978901246872 \\
\hline C & 3.1372 & -2.35921184251247 & -3.740 \\
\hline $\mathrm{Cl}$ & 1.11410 & -0.29404466109281 & -3.849 \\
\hline
\end{tabular}

Table SCZ28. Cartesian coordinates for cycloaddition transition state of the ${ }^{5} \mathrm{H}_{4}$ conformer of 3 with TCAI at the PBE0/DEF2-SVP/D3BJ/CPCM $\left(\mathrm{CH}_{3} \mathrm{CN}\right) / \mathrm{RIJCOSX}$ level.

$\begin{array}{rrrr}\text { C } & 0.03544514900401 & -0.03706519655649 & 0.07943875189604 \\ \text { C } & 0.09752429005356 & -0.18187157930237 & 1.59624049692164 \\ \text { C } & 1.54568883840734 & -0.14293814194658 & 2.10377139951929 \\ \text { C } & 2.37206172053867 & -1.09757480304286 & 1.29048347839803 \\ \text { C } & 2.04991804796122 & -1.29357147916713 & -0.03605194842096\end{array}$


O $\quad 0.93540839421490$

O 2.10946601105560

C 0.30987867590002

O -0.42691256691891

O -0.02688868649106

C 0.25982980874467

C -1.82535650902280

C 1.76464826065699

H -0.95725796006195

H -0.47232897920790

H 1.53438812459849

H 3.42440275434121

H 2.69178761646827

H $\quad-0.29693412364644$

H 1.37186049122799

H 1.33825321693019

H -0.03056527549063

H -0.30269499106976

H -2.31508682245942

H -2.22206642564396

H -2.06922134136768

H 2.29731074894720

H 0.68096344124974

H 2.06950981826697

C 1.77813836089370

O 2.09864923944041

N 1.27763163023348

C 0.32020358887383

C -0.16743607350736

O $\quad-0.21009397184295$

Cl 1.21780968657416

Cl -1.08778173861776

Cl -1.21456055723418
$-0.94924816803069$

1.14179566839638

1.36149790297301

$-1.40540253109291$

1.41092624643741

2.64877562758568

$-1.53187496799253$

2.00934152296372

$-0.33792779526228$

0.65848820400683

$-0.46993891438886$

$-1.22375583119635$

$-1.87942657684214$

2.07439328508576

1.62165241054509

2.88980760429861

2.59457657541756

3.47177659687351

$-0.73089779034125$

$-1.51485840463400$

$-2.50534334038892$

2.95499062306893

2.22048337316863

1.59372073723309

$-2.95488731638692$

$-2.94098111289771$

$-3.62119488401877$

$-4.54755389263919$

$-5.21924744347624$

$-4.82592288440004$

$-5.75636677566849$

$-3.98479226230944$

$-6.59204277407206$
$-0.59976511863471$

2.00508562332365

$-0.42762469397133$

2.03099980136434

$-1.78171578060881$

$-2.37014783076824$

1.99362790869207

3.05688124449729

$-0.28339358408645$

2.03387885610038

3.15914987066603

1.55780763457302

$-0.70179259278907$

0.16550642357163

$-0.26152809923814$

$-2.30883772571115$

$-3.42839354475000$

$-1.88922630026651$

2.57618267604431

0.96363811614439

2.43860133261954

2.88976475287440

3.09139155820933

4.03424425353560

1.99722426082561

3.12955055639405

1.02329250809174

1.27950441894889

$-0.04377248019364$

2.32942834363087

$-1.01151111238713$

$-0.94770421357149$

0.30928320955553

Table SCZ29. Cartesian coordinates for cycloaddition transition state of the ${ }^{4} \mathrm{H}_{5}$ conformer of 3 with TCAI at the PBE0/DEF2-SVP/D3BJ/CPCM $\left(\mathrm{CH}_{3} \mathrm{CN}\right) / \mathrm{RIJCOSX}$ level.

\begin{tabular}{|c|c|c|c|}
\hline C & -0.81276888310642 & 0.32198369383370 & -0.71703922402664 \\
\hline C & .66814394796245 & 06649841404874 & 752139983 \\
\hline $\mathrm{C}$ & 76745087816992 & 10058943953 & 78856 \\
\hline C & 1.26788960749315 & 31006129471 & 261 \\
\hline C & 0.57151900129042 & 2.17807955779128 & 820 \\
\hline $\mathrm{O}$ & 9049260006 & 37201 & 185 \\
\hline $\mathrm{O}$ & 714392251 & 77834 & \\
\hline C & -2.22978965446019 & 17298112113 & 759 \\
\hline $\mathrm{O}$ & 914841105 & 77364371 & 382 \\
\hline $\mathrm{O}$ & 377447956931 & 20450951 & -0. \\
\hline C & 71 & 91 & -0 \\
\hline C & 96 & 327 & \\
\hline C & 68823 & -0.2 & 3.1 \\
\hline $\mathrm{H}$ & -0.1 & 027 & 302 \\
\hline $\mathrm{H}$ & 229997 & 33 & 16 \\
\hline $\mathrm{H}$ & 1.36608 & $-0.6 \varepsilon$ & \\
\hline $\mathrm{H}$ & 1.88 & 2.0 & \\
\hline $\mathrm{H}$ & 0.79377 & 3.22 & -0.4 \\
\hline $\mathrm{H}$ & 0305036 & 248 & 96 \\
\hline $\mathrm{H}$ & -2.5152 & -0.8 & -1.2 \\
\hline $\mathrm{H}$ & -4.564 & 58 & -1.7 \\
\hline $\mathrm{H}$ & -4.7980 & -0.1 & 84 \\
\hline $\mathrm{H}$ & 6605157 & 996 & -0.0 \\
\hline $\mathrm{H}$ & -1.317 & -1.4 & 47 \\
\hline $\mathrm{H}$ & -2.8000 & -1.11411384296179 & 1.98193 \\
\hline
\end{tabular}


H $\quad-2.10362706947561$

H 2.45412675168583

H 2.81056170259239

H 1.97758600947011

C 2.81621627438063

O 3.68785558996709

N 2.33893741490681

C 3.14869241451604

C 2.31174148531092

O 4.34163665693355

Cl 1.07774917829850

Cl 1.52436720424736

Cl 3.34611979198848
$-2.76351651797138$

$-1.25268446866739$

0.49212388191335

$-0.38978948656960$

1.15064323083643

1.89692111308277

0.19413222770645

$-0.53486295727265$

$-1.58427973234337$

$-0.45828936136574$

$-0.72190776402862$

$-2.67835005546127$

$-2.51311864468627$
1.95039316575651

2.71695299742048

2.90400659314324

4.21779848365166

$-0.63596150329532$

$-0.37468543330000$

$-1.32854822537901$

$-2.15357102987852$

$-2.95260837826252$

$-2.29399976072059$

$-3.90094841276794$

$-1.78722737417796$

$-4.03182172523830$

Table SCZ30. Cartesian coordinates for cycloaddition transition state of the ${ }^{5} \mathrm{H}_{4}$ conformer of 4 with TCAI at the PBE0/DEF2-SVP/D3BJ/CPCM(CH $3 \mathrm{CN}) / \mathrm{RIJCOSX}$ level.

\begin{tabular}{|c|c|c|c|}
\hline C & 501 & 920 & \\
\hline C & 5407526403626 & 674515890486 & 0610 \\
\hline C & 371630 & 6101 & 729 \\
\hline $\mathrm{C}$ & 30438196299807 & -0.88679036985039 & 1.40676743839833 \\
\hline C & 06267143375719 & 1896 & 0.079 \\
\hline $\mathrm{O}$ & 9945 & 092 & 3309 \\
\hline $\mathrm{O}$ & 28989550666 & 496249623455 & 853 \\
\hline C & 6042038619 & 1.53625489699047 & 9496 \\
\hline $\mathrm{O}$ & -0.61547065657951 & -1.03946485929655 & 1.85133347 \\
\hline $\mathrm{O}$ & 54542541871 & 2.52500435041409 & -0.0262589 \\
\hline C & 810 & 579 & 995 \\
\hline C & 5522 & 1727 & 0285 \\
\hline C & 39935330079 & 2.33071001269558 & 7043 \\
\hline C & 21029168544 & 01326277996 & 0.24385830 \\
\hline C & 893 & 1931 & 8824 \\
\hline C & 33 & 62 & \\
\hline $\mathrm{H}$ & 336 & 5185 & 0140 \\
\hline $\mathrm{H}$ & 2755 & 0416 & 9018 \\
\hline $\mathrm{H}$ & 706 & 58968045 & 9285 \\
\hline $\mathrm{H}$ & 500 & 31 & 831 \\
\hline $\mathrm{H}$ & 2.7 & 30 & 122 \\
\hline $\mathrm{H}$ & 193 & 769 & 581 \\
\hline $\mathrm{H}$ & 636 & 6515 & 6319 \\
\hline $\mathrm{H}$ & 1230 & 3172 & 5597 \\
\hline $\mathrm{H}$ & 73 & 4.0 & 350 \\
\hline $\mathrm{H}$ & -2 & 960 & 3764 \\
\hline $\mathrm{H}$ & 515 & 7794 & 4365 \\
\hline $\mathrm{H}$ & 1. & & 615 \\
\hline $\mathrm{H}$ & 3409 & 2940 & 984 \\
\hline $\mathrm{H}$ & -0 . & 556 & 3725 \\
\hline $\mathrm{H}$ & -1 & 492 & 444 \\
\hline $\mathrm{H}$ & -0 . & 576 & 1103 \\
\hline $\mathrm{H}$ & 9097 & 68973125678 & 7205 \\
\hline $\mathrm{H}$ & 0039 & 8627 & 8397 \\
\hline $\mathrm{H}$ & -2.6 & 2213 & 163 \\
\hline $\mathrm{H}$ & 2.1 & 83 & 35 \\
\hline $\mathrm{H}$ & 321 & 812 & 5917 \\
\hline $\mathrm{H}$ & 513737196290 & 5598537849 & 8501 \\
\hline C & 22237238671 & -2.6 & 3655 \\
\hline $\mathrm{O}$ & 1.6 & -2.5 & 1498 \\
\hline $\mathrm{N}$ & 235 & & 6153 \\
\hline $\mathrm{C}$ & 0.50645051428805 & -4.57523253670784 & 1.46444136127062 \\
\hline $\mathrm{C}$ & -0.02515221984730 & -5.21058498566666 & 0.14042681008277 \\
\hline $\mathrm{O}$ & 0.25 & 914 & 9331 \\
\hline C & 1.31466684648599 & -5.43206153321028 & מ \\
\hline
\end{tabular}



$\mathrm{Cl}-1.21999180202772$
$-4.07669086996950$
$-0.54324662130097$
Cl -0.79651143575288
$-6.76085017391104$
0.46143223266310

Table SCZ31. Cartesian coordinates for cycloaddition transition state of the ${ }^{4} \mathrm{H}_{5}$ conformer of 4 with TCAI at the PBE0/DEF2-SVP/D3BJ/CPCM $\left(\mathrm{CH}_{3} \mathrm{CN}\right) / \mathrm{RIJCOSX}$ level.

\begin{tabular}{|c|c|c|c|}
\hline $\mathrm{C}$ & -0.36187471253452 & 0.35400440506140 & 00385 \\
\hline C & -0.30251306189288 & 0.05399207239472 & 0.23375196539737 \\
\hline C & 1.05902914403681 & 0.41439568759254 & 0.80505690810629 \\
\hline C & 1.45337725530442 & 1.79483405889794 & 0.32096321327943 \\
\hline C & 0.77148550889915 & 2.37174004220642 & -0.72214966234307 \\
\hline $\mathrm{O}$ & -0.03522734517432 & 1.73675117461734 & -1.52892781288787 \\
\hline 0 & 0.97729332369220 & 0.36202972026869 & 2.20182626600290 \\
\hline C & -1.71544571533199 & 0.08760697219077 & -1.86246665894892 \\
\hline $\mathrm{O}$ & -0.57013001350959 & -1.30891101114864 & 0.39749350464930 \\
\hline $\mathrm{O}$ & -2.69678622663808 & 0.77014755657961 & -1.14152683295759 \\
\hline C & -3.99969210585722 & 0.55400858131753 & -1.62844726730432 \\
\hline C & -1.51600579270137 & -1.61400450332027 & 1.40500102333375 \\
\hline C & 2.22112282632207 & 0.18096442459580 & 2.84458719706020 \\
\hline $\mathrm{C}$ & -4.97842803103438 & 1.31604032635047 & -0.77015025150706 \\
\hline C & -1.65770572103778 & -3.11222373699338 & 06595 \\
\hline C & 1.99538252029062 & 0.12445969739756 & 4.33433903 \\
\hline $\mathrm{H}$ & 0.38455916796820 & -0.25837342715994 & -1.78519514 \\
\hline $\mathrm{H}$ & -1.06259318210768 & 0.67358282663648 & 0.74145356274021 \\
\hline $\mathrm{H}$ & 1.77732221308699 & -0.34239781294777 & 0.43638670207874 \\
\hline $\mathrm{H}$ & 1.94122085024802 & 2.47062 & 6892 \\
\hline $\mathrm{H}$ & 0.90388516911685 & 30046896 & 11356566 \\
\hline $\mathrm{H}$ & -1.884613720 & 77037581 & -1.84226028803319 \\
\hline $\mathrm{H}$ & 4136401553 & 64466489 & 282703628 \\
\hline $\mathrm{H}$ & -4.22946123295465 & -0.53023735424885 & 872404530 \\
\hline $\mathrm{H}$ & -4.06611275421546 & 49650 & $\$ 8625$ \\
\hline $\mathrm{H}$ & -2.48569755 & 190237666969 & 918265 \\
\hline $\mathrm{H}$ & 21392296623 & 74119 & 71346 \\
\hline $\mathrm{H}$ & 2.91060624219199 & 1.00902821139260 & 2.59025808040851 \\
\hline $\mathrm{H}$ & 2.6934 & -0.75 & 0822 \\
\hline $\mathrm{H}$ & -6.00422390 & 65482 & 7413 \\
\hline $\mathrm{H}$ & -4.93475034468002 & 0.96927188294257 & 0.27362867803431 \\
\hline $\mathrm{H}$ & -4.75169892770954 & 2.39287513880141 & -0.78678623211961 \\
\hline $\mathrm{H}$ & -2.38847203745827 & -3.38118425917959 & 2.26846758166551 \\
\hline $\mathrm{H}$ & 2972792509 & 8395 & 46614 \\
\hline $\mathrm{H}$ & -2.00199127086525 & -3.52917 & 34882 \\
\hline $\mathrm{H}$ & 1.32932861994542 & -0.71179086234434 & 4.59577786083785 \\
\hline $\mathrm{H}$ & 2.95126319710695 & -0.01592141011611 & 4.85961026641825 \\
\hline $\mathrm{H}$ & 1.53689755162224 & 1.05799909666168 & 4.69359146451286 \\
\hline C & 3.13731387200242 & 1.58271459022248 & 30200 \\
\hline $\mathrm{O}$ & 3.89463182432667 & 2.43816662528623 & -0.65153930901667 \\
\hline $\mathrm{N}$ & 2.82967625635232 & 0.54725064703963 & -1.60592849827692 \\
\hline C & 3.77853283486699 & -0.14560562962272 & -2.30218614868788 \\
\hline C & 3.12229695658995 & -1.31343878542383 & -3.10466876896240 \\
\hline $\mathrm{O}$ & 4.96880612006323 & 0.03360649826618 & -2.33366797984419 \\
\hline $\mathrm{Cl}$ & 1.89322494826625 & -0.63692033439324 & -4.19800588365057 \\
\hline $\mathrm{Cl}$ & 2.35163967185066 & -2.42336918414194 & -1.94489188716885 \\
\hline $\mathrm{Cl}$ & 4.33516004311466 & -2.18106777280927 & -4.03867114876243 \\
\hline
\end{tabular}

Table SCZ32. Cartesian coordinates for cycloaddition transition state of the ${ }^{5} \mathrm{H}_{4}$ conformer of 12 with TCAI at the PBE0/DEF2-SVP/D3BJ/CPCM $\left(\mathrm{CH}_{3} \mathrm{CN}\right) / \mathrm{RIJCOSX}$ level.

$\begin{array}{llll}\text { C } & 1.94110118603549 & 1.13617485168715 & 0.50206017197932 \\ \text { C } & 1.06041389807185 & 1.09788267562892 & 1.73684189712515 \\ \text { C } & 1.61551746631550 & 1.98259202806686 & 2.86355329841271 \\ \text { C } & 3.07814816867174 & 1.68694589816233 & 3.03675088372457 \\ \text { C } & 3.82943867417181 & 1.36736972171567 & 1.92351942724647\end{array}$


O 3.35071609877169

O 1.45594705458250

C 1.81058748307538

O 0.96307805412070

O 0.46626025177152

Si -0.20011308351197

C -0.24668278548978

C -1.40633566876895

C 0.16347730047465

C 0.01916842507543

C 1.11279559039977

C 0.96250301249449

C -0.28294292220707

C -1.37751768418535

C -1.22516022923938

C -1.25512737167899

C -2.32926443976269

C -3.56873566037999

C -3.72598513001928

C -2.64705191703691

C -1.96174955846568

C -2.89074667280977

C -2.56323611002898

C -0.15638674086378

C 1.25682776935475

C -0.86482880663786

C 0.84241225190816

C 0.43472076644746

C 0.87504879399681

H 1.68922161825615

H 0.07607983078005

H 1.08497443212831

H 3.61065692048156

H 4.92114476664708

H 2.43138705694718

H 2.22559934409377

H -0.48481511924674

H -0.05209536141541

H -0.00917509926081

H -0.61392551806630

H 2.08869458465953

H 1.82601618991363

H -0.39899182631503

H -2.35500428667637

H -2.08240675162219

H -0.28255003716310

H -2.19915439988178

H -4.40996315475771

H $\quad-4.69003263484115$

H -2.76754634312452

H -1.84203750722131

H -2.49228453406057

H -3.05923081777201

H -3.87846605094083

H -2.72847670911842

H -1.91212573235037

H -3.54181755094262

H -0.72739379188573

H 1.78678696355221

H 1.87107591832540

H 1.22837250948376

H $\quad-0.38884573616052$
0.98375094842585

3.35158066368498

2.42548766189488

$-0.23140722099817$

2.65308937547762

3.11572314472023

$-0.54549881807341$

$-0.68272318091359$

3.84952635541501

5.22061913935874

6.09025489089397

7.37121241827055

7.79976481587402

6.93710871202141

5.65430496480327

$-1.41164938882985$

$-1.56974343886559$

$-1.00107281968645$

$-0.27047140580339$

$-0.10836759655623$

3.63917549778629

3.67381946584601

2.82001560613713

1.65465222330597

1.30121340816498

0.42716584599352

4.55510650917428

5.01071811539893

5.72119510923822

0.27002773216456

1.46962339060425

1.74058997354975

2.18382997225074

1.29569800116002

2.31633993588342

3.25723725229892

0.20158473944309

$-1.49988367122372$

3.88530366231544

3.18709993343109

5.75232370120148

8.03922693193117

8.80245930554005

7.25970962835667

4.97633761304240

$-1.85203403628780$

$-2.13750996672695$

$-1.12332993906453$

0.18653782848575

0.47467369984186

4.67651940266218

4.29347612153583

2.66244498475041

4.08330325343182

1.77006078141412

2.81381061347564

3.23153534509040

2.00493541513970

2.16214359507926

0.91945202801993

0.50696066645084

0.09081199818916
0.78341219111425

2.56965217888317

$-0.30686102946512$

2.18945290105735

$-0.58728772443297$

$-2.07853861625870$

2.84654044297520

1.89431652400058

2.82551098772353

2.23066618005549

2.15714635434942

1.62682090833593

1.16594776219895

1.23505331959990

1.76058114356637

0.70818197635748

$-0.16463558441967$

0.13752735749413

1.31484363446154

2.18539611312749

$-1.61973259945431$

$-2.83471969892374$

$-0.47792185687710$

$-3.28681823777830$

$-3.75441983191857$

$-2.71573494073737$

$-2.73487209647063$

$-4.13594609537096$

$-1.74833100479520$

$-0.12545381800463$

1.40823122791029

3.80187529881109

3.85185869451123

1.96535318455113

$-1.21257348580230$

0.28705080989876

3.62439196834048

3.35918717783215

3.92024944957198

2.40551920785759

2.51187256488704

1.57051742720239

0.74710955195668

0.86733510427944

1.79971867816056

0.47221925509505

$-1.08935084299488$

$-0.54944439828494$

1.55216014218580

3.10325826526201

$-1.25375933272964$

$-3.65303285894748$

$-3.23975347420266$

$-2.56268879149110$

$-0.76549739491356$

0.40777337231766

$-0.17546708534342$

$-4.16815859895916$

$-4.19042229342167$

$-2.92222470212974$

$-4.52009032491395$

$-1.78081432758299$ 

H -1.92304302859951
H -0.83084786229622
H 1.86643806914771
H 1.12236435710983
H -0.57644314121516
H 0.43577303182779
H 1.59611142068082
H 1.15382281412919
H $\quad-0.10966227465341$
C 3.03718827028818
O 2.51637789085731
N 3.61001008238847
C 3.17490316119365
C 3.93127726554663
O 2.27139223229456
Cl 3.27101296367647
Cl 3.64243775922385
Cl 5.67235218630147
$-2.48643946158903$
$-3.42459105509602$
$-2.80161377093805$
$-4.51477503853088$
$-4.13576991646211$
$-4.86329296800529$
$-2.06649797717201$
$-0.73171672649112$
$-1.67219073522668$
3.87524247082033
4.92006535732178
3.07519089638471
3.03500146024189
1.93594165691418
3.64791607547009
0.36032764748161
2.13956168165801
1.98586312642300

Table SCZ33. Cartesian coordinates for cycloaddition transition state of the ${ }^{4} \mathrm{H}_{5}$ conformer of $\mathbf{1 2}$ with TCAI at the PBE0/DEF2-SVP/D3BJ/CPCM $\left(\mathrm{CH}_{3} \mathrm{CN}\right) / \mathrm{RIJCOSX}$ level.

\begin{tabular}{|c|c|c|c|}
\hline & -0.26611303137934 & 0.80237674990876 & 0.16927613820882 \\
\hline$C$ & -0.30953767276206 & 0.53297715443545 & 1.67024365901027 \\
\hline C & 1.09856795930723 & 0.49063886662851 & 2.23966689262791 \\
\hline C & 1.87995287738549 & 1.69539028993476 & 1.77541813929961 \\
\hline C & 1.45558339987299 & 2.37844541874501 & 0.66016573242035 \\
\hline $\mathrm{O}$ & 0.53374814581043 & 1.96108677063371 & -0.16130252520254 \\
\hline $\mathrm{O}$ & 1.02574476749153 & 0.40879600305903 & 3.63695073210195 \\
\hline C & -1.64122014437263 & 1.05018042823660 & -0.40556493267267 \\
\hline $\mathrm{O}$ & 9451 & -0.70907579148352 & \\
\hline $\mathrm{O}$ & -1.59833919655352 & 1.08173480195774 & -1.80060093220566 \\
\hline $\mathrm{Si}$ & -2.60854359932932 & 0.17037082237500 & -2.815211628847 \\
\hline C & -1.88008930886758 & -0.78393200384678 & 2.90836753466206 \\
\hline C & -3.22605631971436 & -0.24630858431780 & 2.50122363368728 \\
\hline & & -0.15726950377554 & 4.23 \\
\hline C & 2.43856034332700 & -1.57228488462884 & 3.78497743593014 \\
\hline C & 1.44622363188166 & -2.55094335821538 & 3.92786718494191 \\
\hline C & 1.66647999679496 & -3.85149858372375 & 3.4805 \\
\hline C & 2.88295392937875 & -4.19160606438664 & 2.88350019624935 \\
\hline C & 3.87524129098102 & -3.22467210843890 & 2.73571418173829 \\
\hline C & 3.65069832612100 & -1.92140223438937 & 3.18324169943205 \\
\hline C & -3.59638349636388 & 1.07217162805712 & 2.78964642581731 \\
\hline C & -4.80506519030643 & 1.59028755277984 & 2.3214415983 \\
\hline C & -5.66181153724559 & 0.79005961445254 & 1.56591876079239 \\
\hline $\mathrm{C}$ & -5.30928307800547 & -0.53222908739221 & 1.28599852690605 \\
\hline C & 64939 & -1.04366254505649 & 1.74953705357299 \\
\hline $\mathrm{C}$ & 560204 & -1.66230230315162 & -2.76282381393625 \\
\hline C & 50613 & -1.86991723915093 & -3.162 \\
\hline $\mathrm{C}$ & -2.42220206184689 & -2.32982195071939 & -1.41938468316883 \\
\hline C & -2.33107075764254 & 0.95117144189317 & -4.51888678775669 \\
\hline C & -0.90132012313943 & 1.45324344174466 & 60005806252 \\
\hline C & -2.78065778779790 & 0.03794865843473 & -5.66074989120816 \\
\hline C & 4056948142 & 0.39784229190607 & -2.17947402201735 \\
\hline C & -4.77567190006990 & 1.87119625268716 & -2.10754262194791 \\
\hline $\mathrm{C}$ & -5.41223596085354 & -0.43449941734969 & -2.93642269501446 \\
\hline $\mathrm{H}$ & 0.20353746599204 & -0.05601375624723 & -0.335235458459 \\
\hline & -0.87047859573730 & 1.34487635790997 & 2.16830028785587 \\
\hline $\mathrm{H}$ & 1.56087651066278 & -0.42332784670761 & 1.82577670775583 \\
\hline $\mathrm{H}$ & 2.47036792688181 & 2.25357371948784 & 2.5052178476259 \\
\hline $\mathrm{H}$ & 1.90920187226250 & 3.32331549597397 & 0.34234583671686 \\
\hline $\mathrm{H}$ & -2.01072512740869 & 2.01481338802522 & -0.010546582217 \\
\hline
\end{tabular}



H -2.30620484757406
H -1.49170204407304
0.26533303005716
$-0.25314476145761$
$-0.01188104962945$
3.79146597993993
$-1.85242123373816$
H -1.95607183550965
H 1.98379637361285
H 3.06917655774072
$-0.12574532093287$
0.46437171805870
$-2.28449958326629$
H 0.49094059255190
H 0.88398938870807
H 3.05124847527509
H 4.82154207234195
H 4.42341369540656
H -2.93122815556677
$-4.60609501742347$
$-5.20948608514436$
$-3.47660597395882$
$-1.15907100050578$
1.70058120753343
2.62306176419404
1.19432013462152
H -6.60826424989869
$-1.16571157538067$
3.15648763562747
5.31914270081978
4.04037965785995
4.38811791039970
3.59563165045423
2.52285619621348
2.25188028203718
3.04747821342163
3.38914466256215
2.55041103428668
1.19811360555917
0.70072454005047
$-2.07638157749362$
1.52253476720518
H -3.82038901268485
H -2.76032713952067
H -0.37566439724404
$-2.14126123392726$
$-2.93407328114416$
$-3.52868482790902$
$-3.09611587636256$
H -0.45728155861604
H 0.01898847512999
$-1.53739726812428$
$-1.31532241756169$
$-4.19165165552387$
$-2.49790112717368$
$-1.90649406285285$
$-0.60542907696228$
H -3.47811155857651
H -2.19485765808956
H -2.99449572264213
$-2.22569380860202$
$-3.40898710909507$
1.83686394757082
$-1.12605121845591$
$-1.46072341347769$
$-4.51458753996957$
0.62112996932872
2.00530808047959
$-4.76799686535284$
H -0.81563000422148
H -0.58402221323996
H $\quad-2.15394030986658$
H -2.70228961792897
H $\quad-3.82473541699487$
2.12157102542177
$-5.67940833717082$
$-0.86672938547658$
0.55445405222612
$-3.91406302810641$
$-0.29322689974478$
$-5.72283289333482$
$-6.63264135720222$
$-5.54807490624496$
0.01556122038948 1.99366877452018 2.31439758670996
$-1.14246583873779$
H $\quad-5.75222931259796$
H -4.86513378134804
H -4.04005570612271
H -5.14787311291134
H -5.52062958668915
2.46973981745413
$-1.50338389170362$
$-0.09362138128208$
$-0.35220898046881$
1.01338040286138
C 3.55357975738562
O 4.45028093651292
1.74071217176303
$-0.03689845453104$
N 3.10942909575946
C 3.93763358964509
C 3.20118842706710
O 5.09069029727744
Cl 1.64881433167203
Cl 4.19496145441596
Cl 2.92828820210800
$-1.08462828809268$
$-2.18058992248206$
$-1.23005526337633$
$-2.59519477083337$
$-3.62906806662773$
$-1.49126603524713$
$-1.60826834375061$
$-3.11335725353343$
$-1.54768592601533$
$-2.96425668436180$
$-3.97922217485356$
$-2.46432886667059$
0.73970854478180
0.97321129718089
0.16835989047143
$-0.10117113169059$
$-0.93340402714977$
0.22029397505531
$-0.17903149939373$
$-1.06154462700054$
$-2.55406008618615$

Table SCZ34. Cartesian coordinates for cycloaddition transition state of the ${ }^{5} \mathrm{H}_{4}$ conformer of 15 with TCAI at the PBE0/DEF2-SVP/D3BJ/CPCM $\left(\mathrm{CH}_{3} \mathrm{CN}\right) / \mathrm{RIJCOSX}$ level.
$\begin{array}{ll}\text { C } & 0.47594378707748 \\ \text { C } & -0.39498927652678\end{array}$
C 0.38681926799947
C 1.70600616316713
C 2.31011432557409
O 1.73218035123780
O 0.59043114518066
C 0.85357625806849
O -0.91950969833055
O -0.34263026466422
C -0.22863914707604
C -2.33576425757678
C -0.47478793545550

$-0.19617916818218$

$-0.23809925126349$

0.14712735776673

$-0.57293130195767$

$-0.90250533171260$

$-0.89059159036085$

1.54020142530668

1.20967418856020

$-1.53165410614379$

1.96882413561800

3.27644336817097

$-1.60682633279592$

2.20346996217317
$-0.53417673422778$

0.71193208591297

1.97471837418972

1.98361268627464

0.78409856194379

$-0.37616403852331$

2.05616402328195

$-0.98418068375470$

0.86927951382483

$-1.02819844295719$

$-0.84609902363124$

0.88334746059606

2.69538376846928 
C -0.22917204335964

C -2.93996001320012

C -2.67407965702186

C -3.15958733073769

C -3.91997010479494

C -4.19426612433510

C -3.70242079880843

C 0.98349525257255

C 1.16517575717836

C 0.14132724964541

C -1.06902445682116

C -1.25115650216497

O -1.45117578748457

O 0.79134873731280

C -1.52420203444419

C -2.99209518428910

Cl -3.07513650197824

Cl -3.86200166942686

Cl -3.74448325381430

H -0.04073689106556

H -1.19773939260103

H -0.20472713040824

H 2.38045618619281

H 3.31920339706513

H 1.32068492481448

H 1.55673474782458

H -2.56122037775579

H -2.74519338344830

H -0.61053521828318

H -1.42786799643841

H -2.06868623564512

H $\quad-2.94425896431061$

H -4.29852535034174

H -4.78648442951191

H $\quad-3.90340860044902$

H 1.78062845129783

H 2.11467046666876

H 0.28211642476080

H -1.88095092821690

H -2.20442022237995

H -1.06696640318830

H -1.02766751615031

C 1.14032077152578

O 0.76599865263199

N 1.34693297286896

C 0.52821133050777

C 0.94441898086995

O -0.46197473645528

Cl 2.68895730656059

Cl 0.52577708877209

Cl 0.06399370458504
3.68159669687781

$-0.82662130357695$

$-1.20630456639434$

$-0.44925084416967$

0.69770429313169

1.07989295369039

0.32156105471740

4.25348072369727

5.63667199753801

6.45880268179340

5.88979128616591

4.51130299937125

3.76529505100794

3.90968231202782

5.13419053068215

5.51494528229051

7.21031142720842

4.49157837481412

5.33227749881697

$-0.73872525276682$

0.49790046372010

$-0.18239855558808$

$-0.39479095330380$

$-1.32493086986613$

1.17720135191367

1.66446700411823

$-2.67861681005579$

$-1.25120612875590$

1.79397181767110

2.02634082830212

$-2.09690489614105$

$-0.75282310451676$

1.29287543443034

1.97672040370910

0.63360892820515

3.61053806439034

6.07353815395299

7.54148187476346

6.52555752648234

4.07010535981810

5.32329989880543

5.75556335379900

$-2.43254295084693$

$-2.30708108476316$

$-3.25612161321882$

$-4.33169127471693$

$-5.22179914844461$

$-4.60831483469324$

$-5.53844352698834$

$-4.31957184161441$

$-6.74937848667025$
2.77162371746380

2.02182832469774

3.34481753203547

4.40820131280801

4.16293138526622

2.85073572440286

1.78613978754095

2.38178530699336

2.45304590335974

2.92112192589854

3.32679979017900

3.25239321876850

$-0.66273993052737$

$-0.83844883551834$

$-0.33555482115351$

$-0.27522340745831$

0.24081606701429

0.89163183916356

$-1.87472668538881$

$-1.33696952100720$

0.55554970947758

2.84455529880173

2.82517237074691

0.74537955159391

$-1.97943035321312$

$-0.27290160278579$

0.97779407285323

$-0.07889932335534$

3.71553934288851

2.16205247573736

3.53531340523277

5.43591410908025

4.99770502487908

2.65250708436726

0.75684957707083

2.00687854962020

2.13302069493713

2.96808605177171

3.68818817967296

3.55610361192870

0.64735672293046

$-1.09536510732882$

2.54906455926898

3.66135511570497

1.58445767973207

1.46776899742432

0.25281069800095

2.10435326948890

0.28621434517692

$-1.22901392794714$

0.27797608530482

Table SCZ35. Cartesian coordinates for cycloaddition transition state of the ${ }^{4} \mathrm{H}_{5}$ conformer of 15 with TCAI at the PBE0/DEF2-SVP/D3BJ/CPCM $\left(\mathrm{CH}_{3} \mathrm{CN}\right) / \mathrm{RIJCOSX}$ level.
C 0.64950600416422
C 0.62005372001118
C 1.87662221436287
C 2.20763110945591
C 1.57166656470429
O 0.90016978864744
O 1.62885932866298

$-0.39507980100954$

$-0.34232812605142$

0.31484196268287

1.56063351549584

1.79278453108758

0.89862511339130

0.62848764943416
$-2.10786667196849$

$-0.58414405688802$

$-0.02777778691039$

$-0.82448635468510$

$-2.02099600500652$

$-2.69097749339078$

1.31632938917734 


\begin{tabular}{|c|c|c|c|}
\hline & 50 & 46 & $\begin{array}{r}-2.71141686404740 \\
-0.15987627729415\end{array}$ \\
\hline & & & \\
\hline & -1.68321734886403 & 170044 & \\
\hline & & & 1915 \\
\hline & -0.20058476923367 & 083 & \\
\hline & 2.77693026801519 & 2240942032 & \\
\hline & 2.38401593066997 & & \\
\hline & -1.67556875484384 & -1.59635395056569 & 0.936474599220 \\
\hline & & & \\
\hline & -3.62921873808574 & 344 & \\
\hline & -4.41695414809342 & -1.07404753145838 & 0.65 \\
\hline & & & \\
\hline & -2.47454819060850 & 615719155883 & 502 \\
\hline & & & \\
\hline & 2.952 & & \\
\hline & 1.70 & & \\
\hline & & & \\
\hline & & & \\
\hline & & & \\
\hline & -3.2 & & \\
\hline & & & \\
\hline & & & \\
\hline & & & \\
\hline & & & \\
\hline & & & \\
\hline & & & \\
\hline & -0.2 & & \\
\hline & & & \\
\hline & & & \\
\hline & & & \\
\hline & & & \\
\hline & & & \\
\hline & 0.2 & -1 & \\
\hline & & & \\
\hline & & & \\
\hline & & & \\
\hline & & & \\
\hline & & & \\
\hline & & & \\
\hline & & & \\
\hline & & & -0.4 \\
\hline & & & \\
\hline & & & \\
\hline & & & \\
\hline & & & \\
\hline & 0.4 & & \\
\hline & & & \\
\hline & & & \\
\hline & & & \\
\hline & & & \\
\hline & & & -2.2 \\
\hline & & & \\
\hline & 4.357574887817 & -2.104 & -3 \\
\hline & & & \\
\hline & & & \\
\hline & & & \\
\hline & & & \\
\hline
\end{tabular}


